\title{
Review
}

\section{Past, present and future of nonlinear system identification in structural dynamics}

\author{
Gaëtan Kerschen ${ }^{\mathrm{a}, *}$, Keith Worden ${ }^{\mathrm{b}}$, Alexander F. Vakakis ${ }^{\mathrm{c}, \mathrm{d}}$, \\ Jean-Claude Golinval ${ }^{\mathrm{a}}$
}

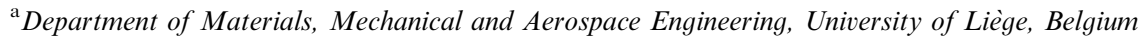

${ }^{\mathrm{b}}$ Dynamics Research Group, University of Sheffield, UK

${ }^{\mathrm{c}}$ Division of Mechanics, National Technical University of Athens, Greece

${ }^{\mathrm{d}}$ Department of Mechanical and Industrial Engineering (adjunct), University of Illinois at Urbana-Champaign, USA

\section{Introduction}

This survey paper contains a review of the past and recent developments in system identification of nonlinear dynamical structures. The objective is to present some of the popular approaches that have been proposed in the technical literature, to illustrate them using numerical and experimental applications, to highlight their assets and limitations and to identify future directions in this research area. The fundamental differences between linear and nonlinear oscillations are also detailed in a tutorial.

Mathematical modeling refers to the use of mathematical language to simulate the behaviour of a 'real world' (practical) system. Its role is to provide a better understanding and characterisation of the system.

\footnotetext{
*Corresponding author.

E-mail addresses: g.kerschen@ulg.ac.be (G. Kerschen), k.worden@sheffield.ac.uk (K. Worden), vakakis@central.ntua.gr, avakakis@uiuc.edu (A.F. Vakakis), jc.golinval@ulg.ac.be (J.-C. Golinval).
} 
Theory is useful for drawing general conclusions from simple models, and computers are useful for drawing specific conclusions from complicated models (Bender, 2000 [1]). In the theory of mechanical vibrations, mathematical models - termed structural models - are helpful for the analysis of the dynamic behaviour of the structure being modeled.

The demand for enhanced and reliable performance of vibrating structures in terms of weight, comfort, safety, noise and durability is ever increasing while, at the same time, there is a demand for shorter design cycles, longer operating life, minimisation of inspection and repair needs, and reduced costs. With the advent of powerful computers, it has become less expensive both in terms of cost and time to perform numerical simulations, than to run a sophisticated experiment. The consequence has been a considerable shift toward computer-aided design and numerical experiments, where structural models are employed to simulate experiments, and to perform accurate and reliable predictions of the structure's future behaviour.

Even if we are entering the age of virtual prototyping (Van Der Auweraer, 2002 [2]), experimental testing and system identification still play a key role because they help the structural dynamicist to reconcile numerical predictions with experimental investigations. The term 'system identification' is sometimes used in a broader context in the technical literature and may also refer to the extraction of information about the structural behaviour directly from experimental data, i.e., without necessarily requesting a model (e.g., identification of the number of active modes or the presence of natural frequencies within a certain frequency range). In the present paper, system identification refers to the development (or the improvement) of structural models from input and output measurements performed on the real structure using vibration sensing devices.

Linear system identification is a discipline that has evolved considerably during the last 30 years (Ljung, 1987 [3]; Soderstrom and Stoica, 1989 [4]). Modal parameter estimation - termed modal analysis — is indubitably the most popular approach to performing linear system identification in structural dynamics. The model of the system is known to be in the form of modal parameters, namely the natural frequencies, mode shapes and damping ratios. The popularity of modal analysis stems from its great generality; modal parameters can describe the behaviour of a system for any input type and any range of the input. Numerous approaches have been developed for this purpose: Ibrahim time domain method (Ibrahim and Mikulcik, 1973 [5]), eigensystem realisation algorithm (Juang and Pappa, 1985 [6]), stochastic subspace identification method (Van Overschee and De Moor, 1996 [7]), polyreference least-squares complex frequency domain method (Peeters et al., 2004 [8]) to cite a few of them. A description of modal analysis is not within the scope of this paper; the interested reader may consult (Heylen et al., 1997 [9]; Maia and Silva, 1997 [10]; Ewins, 2000 [11]) for further details. It is, however, important to note that modal identification of highly damped structures or complex industrial structures with high modal density and large modal overlap are now within reach. Unification of the theoretical development of modal identification algorithms was attempted in (Allemang and Brown, 1998 [12]; Allemang and Phillips, 2004 [13]), which is another sign of the maturity of this research field.

The focus in this overview paper is on structural system identification in the presence of nonlinearity. Nonlinearity is generic in Nature, and linear behaviour is an exception. In structural dynamics, typical sources of nonlinearities are:

- Geometric nonlinearity results when a structure undergoes large displacements and arises from the potential energy. An illustration is the simple pendulum, the equation of motion of which is $\ddot{\theta}+\omega_{0}^{2} \sin \theta=0$; the nonlinear term $\omega_{0}^{2} \sin \theta$ represents geometric nonlinearity, since it models large angular motions. Large deformations of flexible elastic continua such as beams, plates and shells are also responsible for geometric nonlinearities (see, e.g., (Amabili and Paidoussis, 2003 [14]; Nayfeh and Pai, 2004 [15])). An example of a test rig presenting a geometric nonlinearity is shown in Fig. 1. A cantilever beam is connected at its right end to a thin, short beam that exhibits a geometric nonlinearity when large deflections occur.

- Inertia nonlinearity derives from nonlinear terms containing velocities and/or accelerations in the equations of motion, and takes its source in the kinetic energy of the system (e.g., convective acceleration terms in a continuum and Coriolis accelerations in motions of bodies moving relative to rotating frames).

- A nonlinear material behaviour may be observed when the constitutive law relating stresses and strains is nonlinear. This is often the case in foams (White et al., 2000 [16]; Schultze et al., 2001 [17]; Singh et al., 2003 [18]) and in resilient mounting systems such as rubber isolators (Richards and Singh, 2001 [19]). 

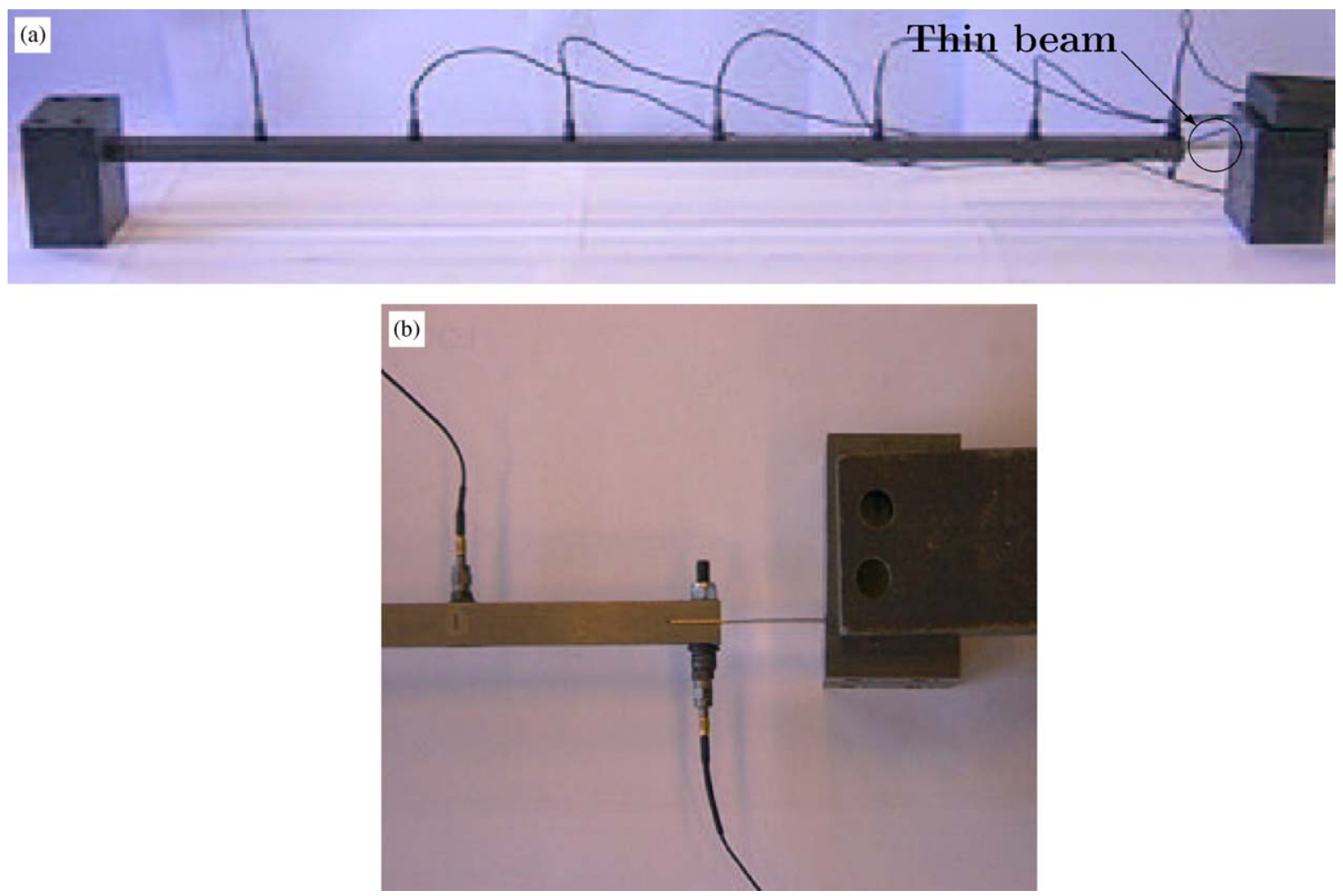

Fig. 1. Cantilever beam connected to a thin, short beam (ECL benchmark; COST Action F3): (a) experimental fixture; (b) close-up of the connection.

- Damping dissipation is essentially a nonlinear and still not fully modeled and understood phenomenon. The modal damping assumption is not necessarily the most appropriate representation of the physical reality, and its widespread use is to be attributed to its mathematical convenience. Dry friction effects (bodies in contact, sliding with respect to each other) and hysteretic damping are examples of nonlinear damping (see, e.g., Caughey and Vijayaraghavan, 1970 [20]; Tomlinson and Hibbert, 1979 [21]; Sherif and Abu Omar, 2004 [22]; Al-Bender et al., 2004 [23]). It is important to note that dry friction affects the dynamics especially for small-amplitude motion, which is contrary to what might be expected by conventional wisdom. For example, the helical wire rope isolators depicted in Fig. 2 are characterised by a softening behaviour (Juntunen, 2003 [24]) with friction within the wire rope, and change of the wire loop geometry when loaded; for this system, the resonant frequency shifts down as the level of excitation is raised, which is a clear indication of nonlinear behaviour.

- Nonlinearity may also result due to boundary conditions (for example, free surfaces in fluids, vibro-impacts due to loose joints or contacts with rigid constraints, clearances, imperfectly bonded elastic bodies), or certain external nonlinear body forces (e.g., magnetoelastic, electrodynamic or hydrodynamic forces). Clearance and vibro-impact nonlinearity possesses non-smooth force-deflection characteristic as shown in Fig. 3 and generally requires a special treatment compared with other types of nonlinearities (Babitsky and Krupenin, 2001 [25]).

Many practical examples of nonlinear dynamic behaviour have been reported in the engineering literature. In the automotive industry, brake squeal which is a self-excited vibration of the brake rotor related to the friction variation between the pads and the rotor is an irritating but non-life-threatening example of an undesirable effect of nonlinearity (Rhee et al., 1989 [26]). Many automobiles have viscoelastic engine mounts which show marked nonlinear behaviour: dependence on amplitude, frequency and preload. In an aircraft, 


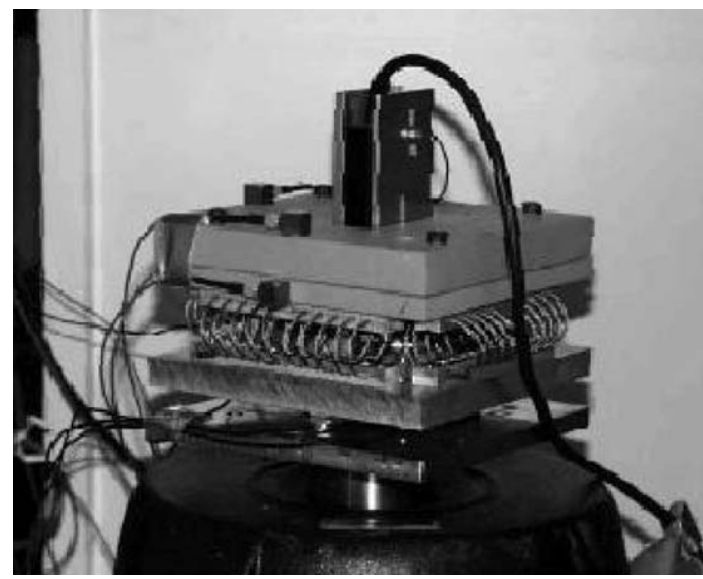

(a)

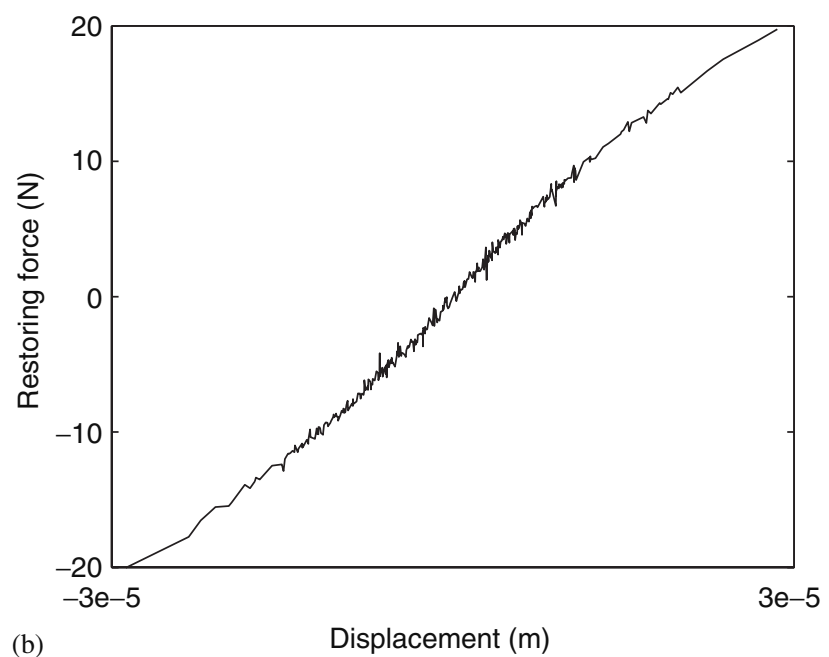

(b)

Fig. 2. Helical wire rope isolators (VTT benchmark; COST Action F3): (a) experimental fixture; the isolators are mounted between the base mass of an electrodynamic shaker and a load mass; (b) measured restoring force.

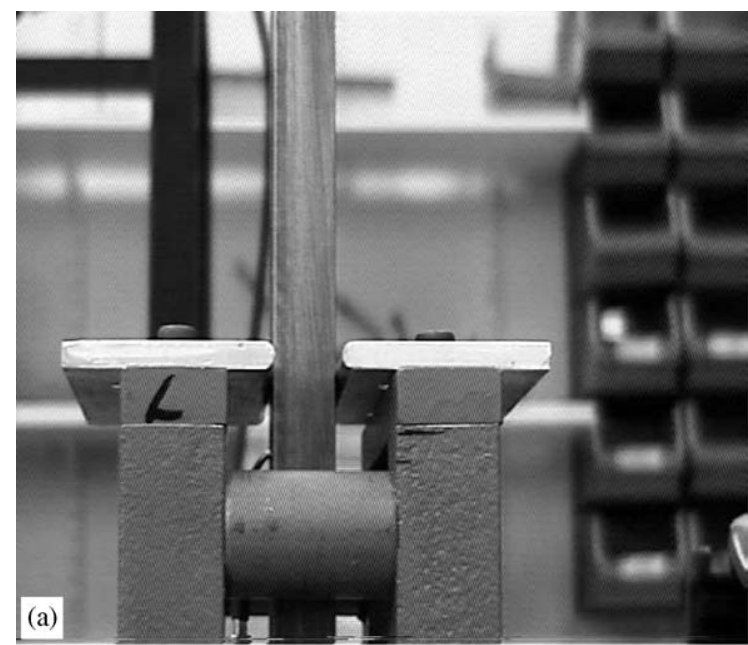

Fig. 3. Impacting beam: (a) experimental fixture; (b) measured restoring force.

besides nonlinear fluid-structure interaction, typical nonlinearities include backlash and friction in control surfaces and joints, hardening nonlinearities in the engine-to-pylon connection, and saturation effects in hydraulic actuators. In (Von Karman, 1940 [27]) a commercial airplane is described in which the propellers induced a subharmonic vibration of order $1 / 2$ in the wings which produced a subharmonic of order $1 / 4$ in the rudder. The oscillations were so violent that the effects on the airplane were catastrophic (Nayfeh and Mook, 1979 [28]). In mechatronic systems, sources of nonlinearities are friction in bearings and guideways, as well as backlash and clearances in robot joints. In civil engineering, many demountable structures such as grandstands at concerts and sporting events are prone to substantial structural nonlinearity as a result of looseness of joints. This creates both clearances and friction and may invalidate any linear model-based simulations of the behaviour created by crowd movement. Nonlinearity may also arise in a damaged structure: fatigue cracks, rivets and bolts that subsequently open and close under dynamic loading or internal parts impacting upon each other. 
With continual interest to expand the performance envelope of structures at ever increasing speeds, there is the need for designing lighter, more flexible, and consequently, more nonlinear structural elements. It follows that the demand to utilise nonlinear (or even strongly nonlinear) structural components is increasingly present in engineering applications. It is, therefore, rather paradoxical to observe that very often linear behaviour is taken for granted in structural dynamics. Why is it so? It should be recognised that at sufficiently smallamplitude motions, linear theory may be accurate for modeling, although it is not always the case (e.g., dry friction). However, the main reason is that nonlinear dynamical systems theory is far less established than its linear counterpart. Indeed, the basic principles that apply to a linear system and that form the basis of modal analysis are no longer valid in the presence of nonlinearity. In addition, even weak nonlinear systems can exhibit extremely interesting and complex phenomena which linear systems cannot. These phenomena include jumps, bifurcations, saturation, subharmonic, superharmonic and internal resonances, resonance captures, limit cycles, modal interactions and chaos. Readers who look for an introduction to nonlinear oscillations may consult (Nayfeh and Mook, 1979 [28]; Strogatz, 1994 [29]; Verhulst, 1999 [30]; Rand, 2003 [31]). More mathematically inclined readers may refer to (Guckenheimer and Holmes, 1983 [32]; Wiggins, 1990 [33]). A brief tutorial which emphasises the important differences between linear and nonlinear dynamics is available in Section 2.1 of this paper.

This is not to say that nonlinear systems have not received considerable attention during the last decades. Even if, for years, one way to study nonlinear systems was the linearisation approach (Caughey, 1963 [34]; Iwan, 1973 [35]), many efforts have been spent in order to develop theories for the investigation of nonlinear systems in structural dynamics. A nonlinear extension of the concept of mode shapes was proposed in (Rosenberg, 1962 [36]; Rosenberg, 1966 [37]) and further investigated in (Rand, 1974 [38]; Shaw and Pierre, 1993 [39]; Vakakis et al., 1996 [40]; Vakakis, 1997 [41]). Weakly nonlinear systems were thoroughly analysed using perturbation theory (Nayfeh and Mook, 1979 [28]; Nayfeh, 1981 [42]; O’Malley, 1991 [43]; Kevorkian and Cole, 1996 [44]). Perturbation methods include for instance the method of averaging, the Lindstedt-Poincaré technique and the method of multiple scales and aim at obtaining asymptotically uniform approximations of the solutions. During the last decade or so, one has witnessed a transition from weakly nonlinear structures to strongly nonlinear structures (by strongly nonlinear systems, a system for which the nonlinear terms are the same order as the linear terms is meant) thanks to the extension of classical perturbation techniques (Chan et al., 1996 [45]; Chen and Cheung, 1996 [46]) and the development of new methodologies (Pilipchuk, 1985 [47]; Manevitch, 1999 [48]; Qaisi and Kilani, 2000 [49]; Babitsky and Krupenin, 2001 [25]).

Recently, a few studies proposed to take advantage of nonlinearities instead of ignoring or avoiding them, which represents an interesting shift in paradigm. For example, the concept of parametric resonance is exploited to design microelectromechanical oscillators with filtering capabilities in (Rhoads et al., 2005 [50]). In (Vakakis and Gendelman, 2001 [51]; Vakakis et al., 2004a [52]; Kerschen et al., 2005b [53]), it is shown that essential (i.e., nonlinearisable) nonlinearity leads to irreversible nonlinear energy transfer phenomena between subsystems - termed nonlinear energy pumping. In (Nichols et al., 2004 [54]), chaotic interrogation and phase space reconstruction are used to assess the strength of a bolted connection in a composite beam. In (Epureanu and Hashmi, 2005 [55]), the geometric shape of dynamic attractors is exploited to enhance small parametric variations in a system.

Focusing now on the development (or the improvement) of structural models from experimental measurements in the presence of nonlinearity, i.e., nonlinear system identification, one is forced to admit that there is no general analysis method that can be applied to all systems in all instances (see, e.g, previous overviews (Adams and Allemang, 1998 [56]; Worden, 2000 [57])), as it is the case for modal analysis in linear structural dynamics. In addition, many techniques which are capable of dealing with systems with low dimensionality collapse if they are faced with system with high modal density. Two reasons for this failure, namely the inapplicability of various concepts of linear theory and the highly 'individualistic' nature of nonlinear systems, are discussed in Section 2.1. A third reason is that the functional $S[\bullet]$ which maps the input $x(t)$ to the output $y(t), y(t)=S[x(t)]$, is not known beforehand. For instance, the ubiquitous Duffing oscillator (Duffing, 1918 [58]), the equation of motion of which is $m \ddot{y}(t)+c \dot{y}(t)+k y(t)+k_{3} y^{3}(t)=x(t)$, represents a typical example of polynomial form of restoring force nonlinearity, whereas hysteretic damping is an example of non-polynomial form of nonlinearity. This represents a major difficulty compared with linear system identification for which the structure of the functional is well defined. 


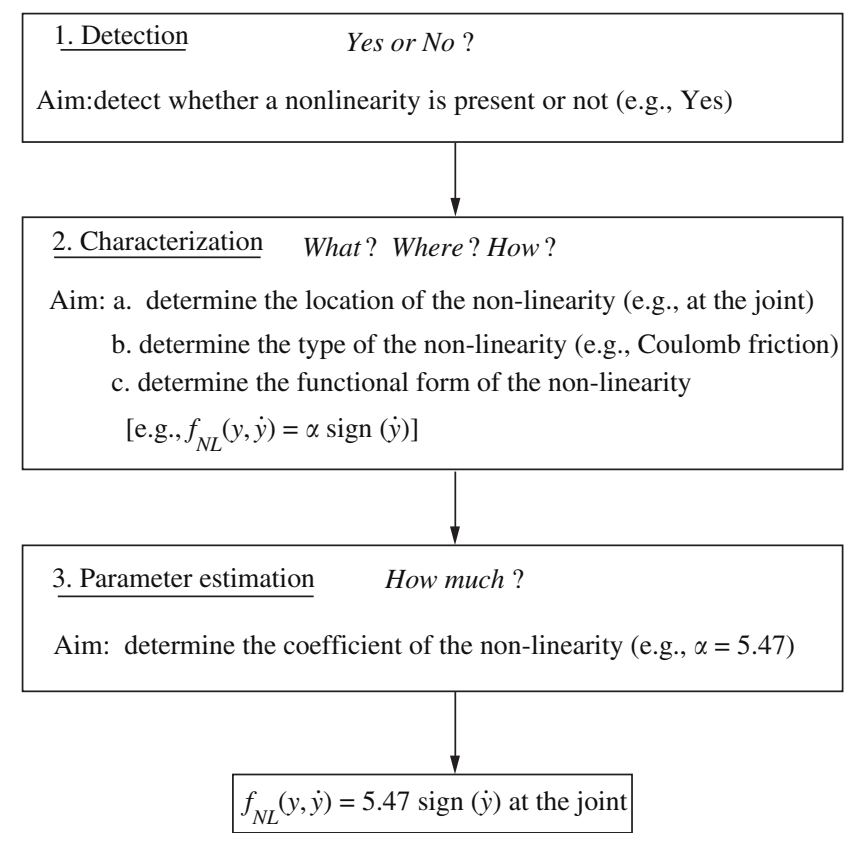

Fig. 4. Identification process.

Even if there is a difference between the way one did nonlinear system identification 'historically' and the way one would do it now, the identification process may be regarded as a progression through three steps, namely detection, characterisation and parameter estimation, as outlined in Fig. 4. Once nonlinear behaviour has been detected, a nonlinear system is said to be characterised after the location, type and functional form of all the nonlinearities throughout the system are determined. The parameters of the selected model are then estimated using linear least-squares fitting or nonlinear optimisation algorithms depending upon the method considered.

Nonlinear system identification is an integral part of the verification and validation (V\&V) process. According to (Roache, 1998 [59]), verification refers to solving the equations correctly, i.e., performing the computations in a mathematically correct manner, whereas validation refers to solving the correct equations, i.e., formulating a mathematical model and selecting the coefficients such that physical phenomenon of interest is described to an adequate level of fidelity. As stated in (Doebling, 2002 [60]), one definition that captures many of the important aspects of model validation is taken from the simulation sciences literature:

The substantiation that a model within its domain of applicability possesses a satisfactory range of accuracy consistent with the intended application of the model (Schlesinger et al., 1979 [61]).

The discussion of verification and validation is beyond the scope of this overview paper; the reader may consult (Roache, 1998 [59]; Link and Friswell, 2003 [62]; Babuska and Oden, 2004 [63]; Hemez et al., 2005 [64]) and references therein.

Scope of the paper: The motivation behind this survey paper is threefold. First, it is meant to provide a concise point of departure for researchers and practitioners alike wishing to assess the current state of the art in the identification of nonlinear structural models. Second, the paper intends to review several methods that have been proposed in the technical literature and to highlight some of the reasons that prevent these techniques from being applied to complex structures. The last goal of this paper is to identify future research needs which would help to 'push the envelope' in nonlinear system identification.

The subject of nonlinear dynamics is extremely broad, and an extensive literature exists. This paper is inevitably biased toward those areas which the authors are most familiar with, and this of course means those areas which the authors and colleagues have conducted research in. Therefore, it is not a comprehensive overview of the past and current approaches for the identification of nonlinear dynamical structures; for instance, there is no attempt to summarise many of the developments originating in control theory. 
Experiment design (e.g, selection of excitation sources, number and location of sensors) which conditions the success of the identification process is not described herein. Some information may be found in (Leontaritis and Billings, 1987 [65]; Duym and Schoukens, 1995 [66]; Worden and Tomlinson, 2001 [67]). System identification in the presence of chaotic vibrations (Moon, 1987 [68]) is not discussed either.

Finally, all systems referenced in this report are assumed to be time-invariant and deterministic, i.e., for given excitation conditions, the system response is always the same without any uncertainty.

\section{Nonlinear dynamics: a tutorial}

\subsection{Dynamics of nonlinear oscillations}

Even though at sufficiently small-amplitude motions nonlinearity may not 'perturb' significantly the linear dynamics, when the energy of the motion increases, stiffness, inertial and/or damping nonlinearities may introduce dynamical phenomena that are radically different than those predicted by linear theory. These distinct dynamical effects must be accounted for in order to accurately understand and robustly model the dynamics.

In fact, in certain practical applications 'nonlinear effects may be unavoidable'; they may affect the dynamics even when the amplitude of the motion is small, contrary to what might be expected by conventional wisdom. Examples are dry friction, strongly nonlinear vibro-impact oscillations and strong geometric nonlinearities that do not admit linearisation even in the small-amplitude regime (e.g, machine components supported in space by configurations of multiple, non-vertical linear springs). To give an example of how common and unavoidable strong nonlinearity can be, consider the vibrations of a system composed of two flexible elements coupled by means of a mechanical joint. A small degeneration of the joint (due to defect or fatigue) may introduce gaps (free-play) between structural components inside the joint, which may generate strong vibro-impact nonlinearities that affect the dynamics over broad frequency ranges (Babitsky and Krupenin, 2001 [25]). To emphasise this last point, it has been shown in (Azeez and Vakakis, 1999 [69]) that even very small free-plays in the bearings of a rotordynamic system lead to strong and potentially catastrophic nonlinear instabilities, evidenced by large-amplitude chaotic motions with frequencies close to linearised critical speeds.

What makes nonlinear dynamics challenging to analyse and model is the well-known result that the principle of linear superposition does not (generally) apply to nonlinear systems. ${ }^{1}$ This means that the system response to any combination of dynamic loads, simultaneously applied, does not equal the sum of the individual responses to each of the loads acting separately. The linear superposition principle is the cornerstone of linear theory, and, probably, the main reason why this theory is so well developed and applied to many fields of science and engineering. Of course, this does not necessarily mean that 'linear problems are easy to analyse', given, for example, the current limitations of quasi-linear theory (there is no complete theory for linear ordinary or partial differential equations with general time-dependent coefficients, though the case of periodically varying coefficients is addressed by Floquet theory (Yakubovich and Starzhinskii, 1975 [73])).

Based on the linear superposition principle, various concepts, theories and methods for analysing the dynamics and performing system identification of linear systems have been developed, including,

- Convolution integrals, or more generally, the theory of Green's functions.

- Frequency response functions (FRFs) for vibration analysis and modal analysis in the frequency domain, or more generally, the theory of linear integral transforms (Laplace, Fourier, Hankel and others); timedomain modal analysis methods.

- The theory of linear operators and spectral theory.

- Linear boundary value problems (BVPs), Sturm-Liouville problems, eigensolutions, and the theory of orthogonal functions; eigenvector/eigenfunction expansions of response vectors/functions in finite/infinitedimensional eigenspaces spanned by complete sets of eigensolutions of appropriately formulated linear BVPs.

\footnotetext{
${ }^{1}$ It is noted that attempts for developing nonlinear superposition principles have been made (e.g., Bäcklund transformations (Jackson, 1990 [70]) and (Ames, 1978 [71]; Anderson et al., 1982 [72])).
} 
These concepts which are purely linear constructions cannot be directly applied to nonlinear problems. For example, no invariance or even strict definition of FRFs applies for nonlinear vibration because harmonic external forces typically generate multi-harmonic nonlinear responses, rendering the concept of FRF invalid. Another example is that linear constructions such as regular convolution integrals do not hold in nonlinear theory, though higher-order convolution operators can be defined and the nonlinear response expressed in terms of Volterra series expansions and higher-order FRFs (HOFRFs). This does not mean that these linear methods cannot be indirectly applied to certain classes of nonlinear problems through, for example, perturbation techniques, whereby the nonlinear problems are reduced to a hierarchy of linear subproblems where the aforementioned techniques can be applied (see for example, (Nayfeh and Mook, 1979 [28]; Vakakis et al., 2004b [74])).

The inapplicability of traditional, well-established linear techniques to nonlinear theory poses serious technical challenges to the development of nonlinear system identification techniques capable of (i) reliably identifying the (predominant) nonlinearities present in the measured dynamics; (ii) providing accurate measures of these nonlinearities; (iii) creating models that correctly regenerate the measured dynamical response; and (iv) being applicable to a wide class of nonlinear dynamical systems. A first step toward addressing these challenges is the discussion of the basic features that distinguish the linear from the nonlinear dynamics. Of course, given our incomplete knowledge of nonlinear dynamics (especially in higher dimensions) and the highly individualistic nature of nonlinear systems that prevents the formulation of general theories encompassing wide classes of nonlinear dynamical systems, any attempt for such broad a discussion is destined to be non-exhaustive.

A first typical dynamical feature of the nonlinear response is the frequency-energy dependence of free oscillations. In other words, the frequency of motion of an unforced nonlinear oscillator with hardening or softening characteristics increases or decreases with amplitude, respectively (Nayfeh and Mook, 1979 [28]). This frequency-energy dependence is a basic deviation from linear vibration theory which predicts amplitudeindependent natural frequencies. It is the cause of many features in the dynamics, such as Liapunov (but not necessarily orbital) instability of the free periodic responses of undamped nonlinear oscillators (Nayfeh and Mook, 1979 [28]; Minorsky, 1983 [75]); the non-existence of analytic solutions for free damped nonlinear responses (Panayotounakos et al., 2002 [76]); and complex nonlinear phenomena in the unforced dynamics, such as nonlinear mode localisation in periodic arrays of nonlinear oscillators (Vakakis et al., 1996 [40]).

One way to make a transition between linear and nonlinear dynamics is through the extension of the concept of normal mode of classical linear vibration theory to nonlinear systems. In particular, the concept of nonlinear normal mode (NNM) has been introduced (Rosenberg, 1966 [37]; Vakakis et al., 1996 [40]; Vakakis, 1997 [41]). Although such a notion might seem self-contradictory in view of the inapplicability (in general) of the principle of linear superposition to nonlinear systems, it has been shown that employing the concept of NNMs one can better understand and explain the free and forced dynamics of nonlinear oscillators. For example, complex dynamic interactions occurring in systems of coupled oscillators, such as nonlinear localisation of energy in space (Vakakis et al., 1996 [40]) and irreversible nonlinear energy transfer phenomena between subsystems - termed nonlinear energy pumping - (Vakakis and Gendelman, 2001 [51]; Vakakis et al., 2004a [52]; Kerschen et al., 2005b [53]), have been studied in a framework of NNMs. Given that the classical theory of Sturm-Liouville BVPs cannot be directly extended to nonlinear theory, alternative ways have been developed to define rigorously NNMs of discrete or continuous nonlinear oscillators.

An obvious phenomenological and formal definition of a NNM is through the nonlinear extension of the concept of normal mode of classical vibration theory. In that context one defines a NNM of an undamped discrete or continuous system as a synchronous (vibration in-unison) periodic oscillation where all material points of the system reach their extreme values and pass through zero simultaneously. ${ }^{2}$ Clearly, when a discrete system vibrates on a NNM the corresponding oscillation is represented by a line in its configuration space, termed modal line. Linear systems possess straight modal lines since their coordinates obey linear relations during normal mode oscillation. The modal lines of nonlinear systems are typically curves, though when special symmetries are obeyed these curves degenerate to straight lines as in the linear case.

\footnotetext{
${ }^{2}$ When internal resonances occur, the previous NNM definition is not valid in the configuration space of the system, but rather in an appropriately defined modal space as discussed in (King and Vakakis, 1996 [77]; Vakakis et al., 1996 [40]).
} 
A unique feature of modal curves of nonlinear systems is their energy dependence, which is in similarity to the energy dependence of the corresponding frequency of oscillation. This means that the nonlinear mode shapes change with varying energy (however, no such dependence exists when the nonlinear modal lines degenerate into straight lines due to special symmetries). The energy dependence of the nonlinear modal curves prevents the direct separation of space and time in the governing nonlinear equations of motion, in contrast to linear theory. This, in turn, prevents the computation of the modal curves of an undamped nonlinear oscillator by solving a BVP (again in contrast to the linear case). Instead, a singular perturbation problem is formulated, which coupled with a double-expansion perturbation method, leads to a hierarchy of linear BVPs, the solutions of which provide analytic approximations to the modal curves (Vakakis et al., 1996 [40]). We mention here the alternative definition of Shaw and Pierre (1991 [78], 1993 [39]) and of A.H. Nayfeh and S.A. Nayfeh (1994 [79], 1995 [80]), who defined NNMs as invariant manifolds in the phase space of the system. This alternative definition enables the direct extension of the concept of NNM to damped oscillators, although extension of 'undamped' NNMs to the weakly damped case is also possible.

Another distinct and intriguing feature of NNMs is that they can exceed in number the degrees of freedom (DOFs) of a discrete oscillator. This is due to NNM bifurcations that lead to mode instabilities (a concept that is foreign to linear theory), and bifurcating branches of nonlinear localised modes. Such mode bifurcations may occur even in simple mechanical oscillators with a small number of DOFs (Vakakis et al., 1996 [40]); so essentially nonlinear vibration modes with no counterparts in linear theory may be encountered more often in practical applications than intuition may suggest. These bifurcations should be taken into account in studies of system identification and order reduction of nonlinear dynamical systems.

Starting from the area of system identification and modal analysis, traditional techniques for analysing the dynamics of nonlinear structures are based on the assumptions of weak nonlinearities and of a 'nonlinear' modal structure that is similar or small perturbation of the underlying linearised system. The previous discussion shows that this simplified approach to nonlinear system identification may lead to erroneous results when mode bifurcations occur, that generate additional, essentially nonlinear modes of vibration with no counterparts in linear theory. In this context, the concept of NNM provides a valuable tool for understanding the effects of structural nonlinearities on the dynamics, and for developing truly nonlinear system identification - modal analysis methodologies that take into account the possibilities of modal interactions and modal spaces that are distinctly different than those predicted by classical linear vibration theory.

In general, bifurcations of equilibrium positions or periodic orbits of nonlinear systems are the source of additional distinctively nonlinear features in the dynamics. A bifurcation takes place when qualitatively different dynamics occur in the neighbourhood of a point in parameter space, and is associated with the failure of analytic continuation of a solution branch at that point. In mathematical terms, a bifurcation corresponds to the failure of the implicit function theorem at this point in parameter space (Guckenheimer and Holmes, 1983 [32]; Wiggins, 1990 [33]) or equivalently with the non-invertibility of the Jacobian of the linearised dynamical system at this point. Among essentially nonlinear dynamics caused by bifurcations we mention:

- The possibility of multiple, co-existing stable equilibrium positions (in contrast to linear systems that possess only a single equilibrium), each with its own separate domain of attraction (to which one the motion is eventually attracted depends on the initial conditions). An example of co-existing stable solutions is given in the frequency-energy diagram of Fig. 9 where multiple NNMs may co-exist at specific frequency and energy ranges.

- Sudden nonlinear transitions between stable attractors (jumps) caused by nonlinear hysteresis phenomena. These transitions can be especially dangerous when a linear-like (trivial) stable equilibrium co-exists in a certain frequency range with an essentially nonlinear (finite-amplitude) stable equilibrium. This is the case, for example, in forced resonances of systems with cubic nonlinearities (Nayfeh and Mook, 1979 [28]). In Fig. 5 we present a nonlinear hysteresis loop in the fundamental resonance of a mode with softening cubic nonlinearities (the frequency of the periodic excitation is nearly identical to the linearised natural frequency of the mode). As a result of nonlinear hysteresis there occur sudden transitions (jumps) between co-existing stable branches of solutions, which appear as sudden changes in the amplitude and phase of the motion for small variations of the forcing frequency. These jump phenomena are caused by small variations of the 


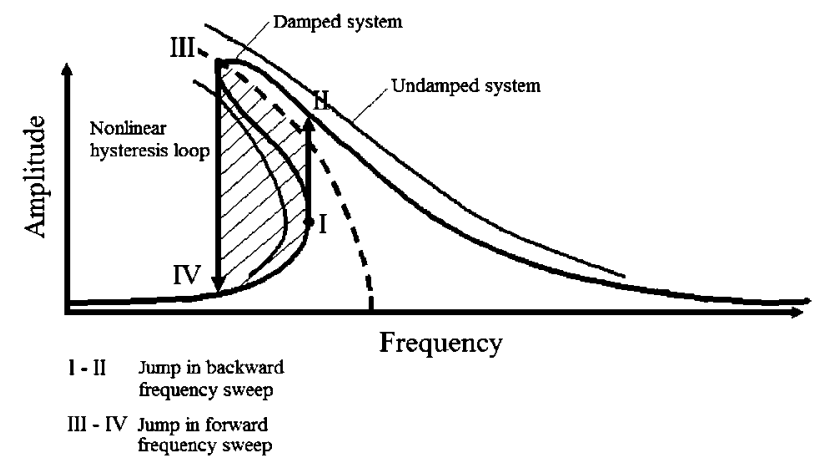

Fig. 5. Nonlinear jump phenomena in the fundamental resonance of a mode with softening cubic nonlinearity.

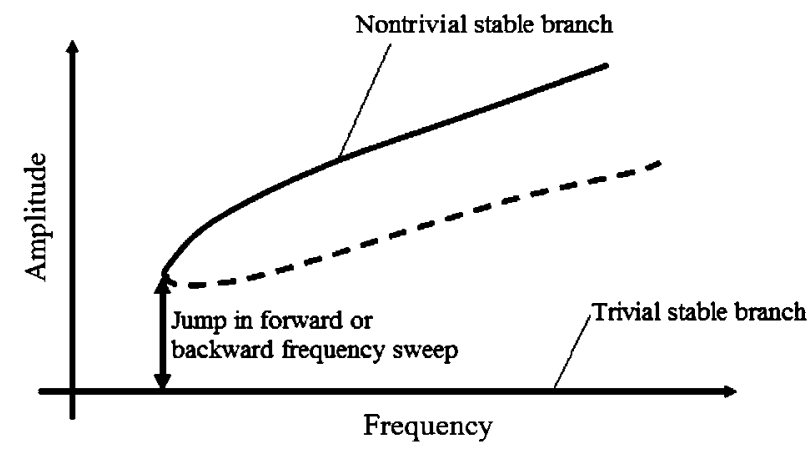

Fig. 6. Nonlinear jump phenomenon in the subharmonic resonance of a mode with stiffening cubic nonlinearity.

initial conditions of the motion which may drift the response between competing domains of attraction of stable solutions in the phase space of the system. Note that the forward frequency jump III-IV is sensitive to damping, since lighter damping shifts downward the frequency where this jump occurs. Such nonlinear transitions are typical in forced resonances of nonlinear oscillators. This type of transitions between coexisting stable branches is not always associated with nonlinear hysteresis phenomena. In Fig. 6 a jump in the subharmonic resonance of a mode with hardening cubic nonlinearity is depicted (the frequency of the external excitation is nearly one third the linearised natural frequency of the mode (Nayfeh and Mook, 1979 [28])). In this case, there is a sudden transition between a trivial and a non-trivial subharmonic solution for forward or backward frequency sweeps. It is interesting that the trivial subharmonic solution corresponds to the linearised response of the mode, meaning that the nonlinear subharmonic resonance may appear suddenly 'out of nowhere' for small variations of the initial conditions when the frequency of the external force is in the range of co-existing subharmonic solutions. As a result the structure may experience severe levels of unwanted (and unaccounted) vibrations.

- Transitions of regular motions to chaotic, where the dynamics seem to be unpredictable, irregular and random-like, possessing sensitivity to initial conditions. It has been established in the literature that chaotic motions occur even in nonlinear oscillators with relatively simple configurations, such as strange attractors in forced, single-degree-of-freedom (SDOF) vibro-impact oscillators (Shaw and Holmes, 1983 [81]), in forced, single-mode vibrations of beams in magnetic fields (Moon and Holmes, 1979 [82]) and in as simple systems as bouncing balls on moving barriers (Holmes, 1982 [83]). Hamiltonian chaos may also occur during the free vibrations of $n$-DOF $(n \geqslant 2)$ non-integrable Hamiltonian oscillators (Vakakis and Rand, 1992 [84]).

- Chaotic explosions where for a small parameter change a regular dynamic response 'explodes' to chaotic behaviour introducing a global form of instability in the dynamics. Such chaotic explosions might often 


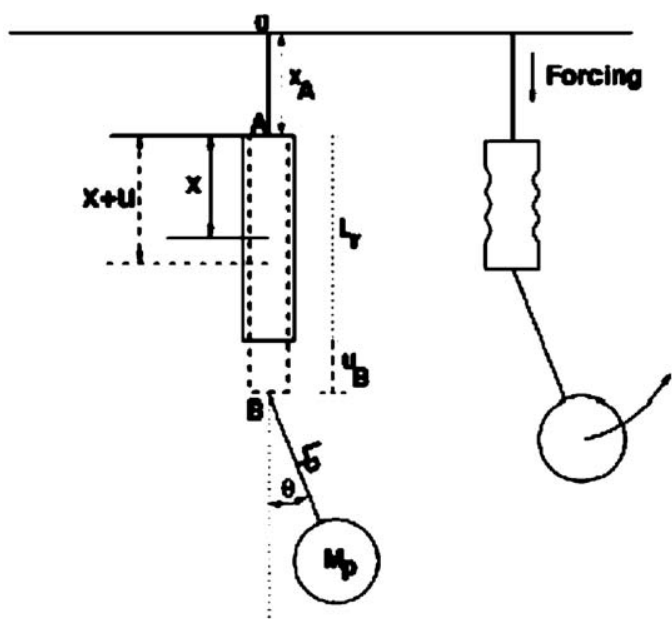

(a)

Fig. 7. (a) Rod-pendulum system; (b) chaotic explosion for increasing forcing amplitude (Schwartz et al., 2004 [85]).

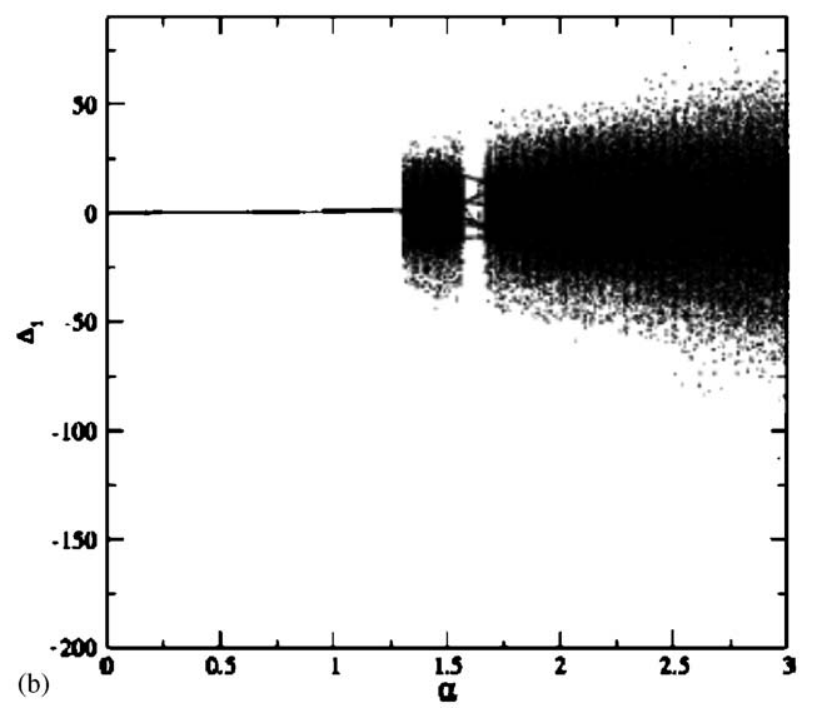

ppear in flexible assemblies designed to operate in the linear regime when a certain component degenerates due to fatigue or defect (for example, (Azeez and Vakakis, 1999 [69]) shows that in rotordynamic systems with a small clearance defect in one of the bearings small variations of the frequency of rotation may lead to chaotic explosions with no precursors (cascade of bifurcations)). Another example of a chaotic explosion is given in (Schwartz et al., 2004 [85]), where the oscillations of the rod-pendulum system of Fig. 7(a) were studied. The rod is forced from the top harmonically with frequency $\Omega$ and amplitude $\alpha$, and possesses complicated dynamics. In Fig. 7(b) the numerical Poincaré map of a measure of the rod displacement $\Delta_{1}$ for varying forcing amplitude $\alpha$ under fixed frequency $\Omega$ is depicted (for fixed $\alpha$ the Poincare map is constructed by plotting on the same vertical line the values of $\Delta_{1}$ at integer multiples of the period of the forcing). Note the sudden loss of stability of the periodic orbit above $\alpha \approx 1.29$ when sudden onset of chaos occurs. Such chaotic explosions occur in multi- or infinite-DOF nonlinear systems, and are characterised by the sudden appearance of unstable chaotic motions for small changes of the control parameters.

- For small parameter changes, transitions from one type of motion to a qualitatively different one may occur. This is the case, for example, of the supercritical Hopf bifurcation (Guckenheimer and Holmes, 1983 [32]; Wiggins, 1990 [33]) when for a small parameter change a stable linear-like (trivial) equilibrium looses stability and gives rise to a stable limit cycle motion (clearly such bifurcations can become especially dangerous if left unaccounted for in the system design).

- Increase of the dimensionality of the dynamics when a bifurcation occurs. This means that the attractor of the dynamics after the bifurcation is realised in a higher-dimensional subspace of the phase space of the system, compared to the attractor before the bifurcation (such dimensionality increases are captured nicely by the method of proper orthogonal decomposition (POD) as shown in (Cusumano et al., 1994 [86]; Azeez and Vakakis, 2001 [87])).

Essentially nonlinear phenomena such as the aforementioned ones are caused by nonlinear energy interactions due to internal resonances (IRs), or transient/sustained resonance captures (TRCs/SRCs). IRs are nonlinear beat phenomena whereby two or more nonlinear modes with commensurable linearised natural frequencies exchange energy between them in a reversible or irreversible fashion (Nayfeh and Mook, 1979 [28]). The only analog in linear theory is the classical beat phenomenon where two modes with nearly equal natural frequencies exchange reversibly energy between them. IRs increase the dimensionality of the dynamics and are the cause of many interesting dynamic phenomena in nature (MacKay and Meiss, 1987 [88]), ranging from orbital mechanics (gaps in distributions of asteroids (Dermott and Murray, 1983 [89]), chaotic rotation of Hyperion (Wisdom et al., 1984 [90])), to nuclear physics (Gerasimov et al., 1986 [91]), 
and chaotic transport and mixing in fluids (Wiggins, 1992 [92]). In mechanical systems, IRs are responsible for virtually every strongly nonlinear phenomenon, ranging from bifurcations to nonlinear localisation and chaos (Guckenheimer and Holmes, 1983 [32]; Wiggins, 1990 [33]). We mention merely the very interesting high- to low-frequency energy transfers in flexible systems (Nayfeh and Mook, 1995 [93]; Malatkar and Nayfeh, 2003b [94]), caused by IRs between the amplitude modulations of high- and low-frequency modes. Clearly, such energy exchanges between modes with well-separated frequencies are not possible in linear theory.

Resonance captures (RCs) occur when the dynamics of a nonlinear system are 'captured' on a resonance manifold defined by an integrable relationship between the frequency of the mode and a different frequency. The capture of the dynamics on the resonance manifold can be either sustained (SRC), or transient (TRC); in the latter case after a finite duration of capture the dynamics 'escape' from the neighbourhood of the resonance manifold (Quinn, 1997 [95]; Zniber and Quinn, 2003 [96]). It has been shown that RC leads to interesting dynamic phenomena, including irreversible transfer of energy between different components of a mechanical system (Kerschen et al., 2005b [53]; Lee et al., 2005 [97]). The previous discussion leads to the conclusion that any attempt to apply traditional linear system identification techniques to capture the dynamics of mechanical system possessing such phenomena is bound to failure.

Considering the steady state responses of nonlinear systems to harmonic excitations, IRs or RCs lead to forced resonances, which, apart from the case of fundamental resonance (i.e., strong steady response at the frequency of the external excitation), have no counterparts in linear dynamics: subharmonic, superharmonic, combination, or autoparametric resonances. Because of the capacity of a nonlinear system to generate multiple harmonics subject to single harmonic input, it is possible to obtain strong steady state nonlinear response at frequencies considerably apart from the forcing frequency (Nayfeh and Mook, 1979 [28]). It follows that the study of the harmonics of the response can be a useful tool for identifying the essentially nonlinear properties of a system. Indeed, it is exactly this multi-harmonic content of the steady state nonlinear output that prevents the extension of the concept of FRF to nonlinear systems (though approximate methods based on linearisation have been developed leading to describing functions, i.e., approximate nonlinear FRFs). In addition, for systems composed of linear 'primary' systems nonlinearly coupled to 'secondary' subsystems, there is the possibility of autoparametric resonance, where the linear mode of the primary system looses stability through nonlinear interaction with the secondary subsystem (Tondl et al., 2000 [98]). Again, such structural instabilities generated through nonlinear energy interactions between components of the system cannot occur in linear settings, and can be dangerous if left unaccounted for at the design stage.

Some basic, distinctively nonlinear features of forced and unforced dynamic responses with no counterparts in linear theory have been discussed. These features differentiate clearly the nonlinear from the linear dynamics, and, hence, can form the basis for developing efficient nonlinear system identification techniques. The efficacy of any nonlinear system identification technique should be judged by the degree to which it addresses at least a subset of these essentially nonlinear features (it would be unrealistic to expect that a method could be capable of identifying all possible nonlinear phenomena given the highly individualistic nature of nonlinear systems). In addition to detecting and identifying qualitatively and quantitatively broad classes of nonlinear phenomena, the reliability and robustness of nonlinear system identification would be enhanced if it leads to mathematical models that can accurately and robustly regenerate the measured response.

\subsection{Complicated dynamics of a two degree-of-freedom nonlinear system: a nonlinear normal mode perspective}

\subsubsection{Dynamics of the undamped system}

In this section, the dynamics of a two-DOF system comprised of a linear oscillator coupled by means of an essentially nonlinear stiffness to a small-mass attachment is discussed; the system is depicted in Fig. 8. Through this example, the objective is to demonstrate that even nonlinear systems of very simple configuration can possess surprisingly complicated and rich dynamics. It is also shown that through the use of NNMs the dynamics of this system can be systematically explored and understood. Moreover, the knowledge of the structure of periodic solutions of the undamped system can be used to understand complex multi-frequency transitions in the transient damped dynamics. A more detailed discussion of the dynamics of this system can 


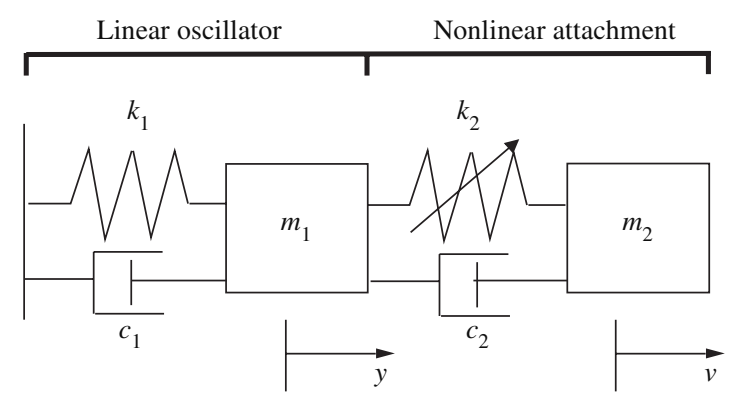

Fig. 8. The two-DOF system with essential stiffness nonlinearity.

be found in (Kerschen et al., 2005b [53]; Lee et al., 2005 [97]), where additional dynamical features such as passive energy transfer are studied.

The equations of motion of the system are given by

$$
\begin{aligned}
& m_{1} \ddot{y}+c_{1} \dot{y}+c_{2}(\dot{y}-\dot{v})+k_{1} y+k_{2}(y-v)^{3}=0, \\
& m_{2} \ddot{v}+c_{2}(\dot{v}-\dot{y})+k_{2}(v-y)^{3}=0 .
\end{aligned}
$$

The variables $\omega_{0}^{2}=k_{1} / m_{1}, C=k_{2} / m_{1}, \varepsilon=m_{2} / m_{1}, \lambda_{1}=c_{1} / m_{1}, \lambda_{2}=c_{2} / m_{1}$ are introduced

$$
\begin{aligned}
& \ddot{y}+\lambda_{1} \dot{y}+\lambda_{2}(\dot{y}-\dot{v})+\omega_{0}^{2} y+C(y-v)^{3}=0, \\
& \varepsilon \ddot{v}+\lambda_{2}(\dot{v}-\dot{y})+C(v-y)^{3}=0 .
\end{aligned}
$$

The periodic orbits of the undamped system (2) (i.e., with $\lambda_{1}=\lambda_{2}=0$ ) are studied for small values of $\varepsilon$, that is, for small-mass nonlinear attachments.

In Fig. 9, the various branches of periodic solutions for parameters $\omega_{0}=C=1.0, \varepsilon=0.05$ are presented in a frequency-energy plot. There are two general classes of solutions: symmetric solutions Snm \pm correspond to orbits that satisfy the initial conditions $\dot{v}(0)= \pm \dot{v}(T / 2)$ and $\dot{y}(0)= \pm \dot{y}(T / 2)$, where $T$ is the period, $n$ is the number of half-waves in $v$, and $m$ the number of half-waves in $y$ in a half-period interval; unsymmetric solutions $U n m \pm$ are orbits that fail to satisfy the initial conditions of the symmetric orbits, with the same notation for the two indices. A frequency index is assigned to a specific branch of solutions and is equal to the ratio of its indices; e.g., $S 21 \pm$ is represented by the frequency index $\omega=2 / 1=2$. This convention holds for every branch except $S 11 \pm$, which, however, are particular branches because they form the basic backbone of the entire plot. On the energy axis the (conserved) total energy of the system when it oscillates in the corresponding periodic motion is depicted. Transitions between certain branches represented by dashed lines in Fig. 9 seem to involve 'jumps', but this is only due to the frequency convention adopted; no actual discontinuities in the dynamics occur (by their definition, branches $S(k n)(k m) \pm, k$ integer, are identified with $S n m \pm$ ).

Four elements of the frequency-energy plot are described in what follows: (a) the backbone of the plot; (b) the branches of symmetric solutions; (c) the branches of unsymmetric solutions and (d) the special orbits.

The backbone of the frequency-energy plot is formed by the branches $S 11+$ and $S 11-$, which represent inand out-of-phase NNMs possessing one half-wave per half-period, respectively. The natural frequency of the linear oscillator $\omega_{0}=1$ (which is identified with a frequency index equal to unity, $\omega=1$ ) naturally divides the periodic solutions into higher- and lower-frequency modes. A close-up of $S 11+$ branch is presented in Fig. 10 together with some modal curves depicted in the configuration plane $(y, v)$ of the system. The horizontal and vertical axes in the configuration plane are the nonlinear and linear oscillator responses, respectively, and the aspect ratios in these plots are set so that equal tick mark increments on the horizontal and vertical axes are equal in size, enabling one to directly deduce whether the motion is localised in the linear or the nonlinear oscillator. Fig. 10 clearly highlights the energy dependence of the NNMs; the NNMs become strongly localised to the nonlinear attachment as the total energy in the system decreases.

There is a sequence of higher- and lower-frequency periodic solutions bifurcating or emanating from branches $S 11 \pm$, which are denoted as tongues. Each tongue occurs in the neighbourhood of an IR between the linear oscillator and the nonlinear attachment, and corresponds to either symmetric ( $S$-tongue) or 


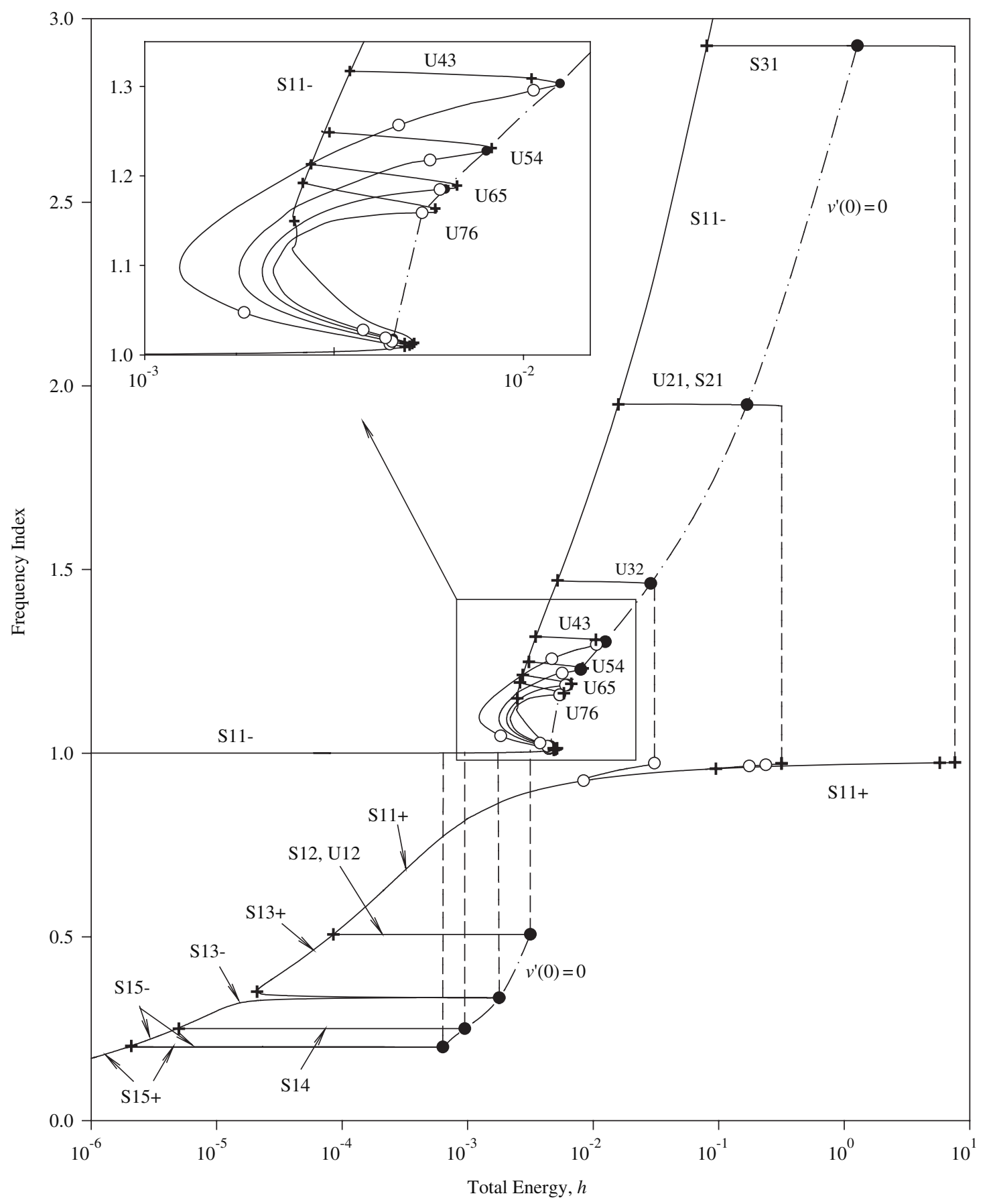

Fig. 9. Frequency-energy plot of the periodic orbits; for the sake of clarity no stability is indicated; symbols indicate bifurcation points (stability-instability boundaries): ( + ) four Floquet multipliers at +1 , and ( $\circ$ ) two Floquet multipliers at +1 and two at -1 ; special orbits are denoted by bullets $\bullet$ (Lee et al., 2005 [97]).

unsymmetric ( $U$-tongue) periodic motion of the system. For example, the subharmonic NNMs on tongues $S 13 \pm$ correspond to motions where the linear oscillator oscillates 'three times faster' than the nonlinear attachment. A close-up of branches $S 13 \pm$ is shown in Fig. 11 and is another illustration of the energy 


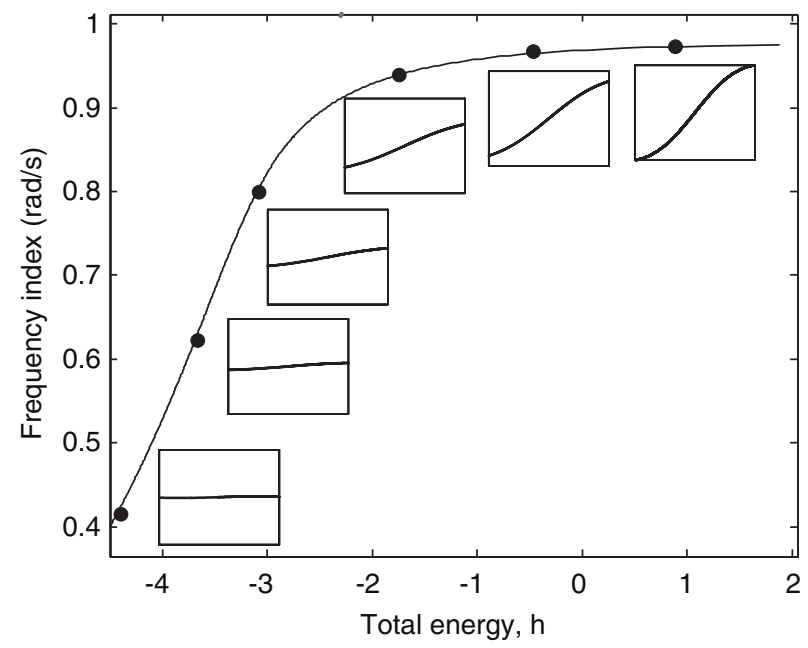

Fig. 10. Close-up of $S 11+$ branch in the frequency index-logarithm of energy plane; at certain points of the branch the corresponding motions in the configuration plane $(y, v)$ are depicted (Lee et al., 2005 [97]).

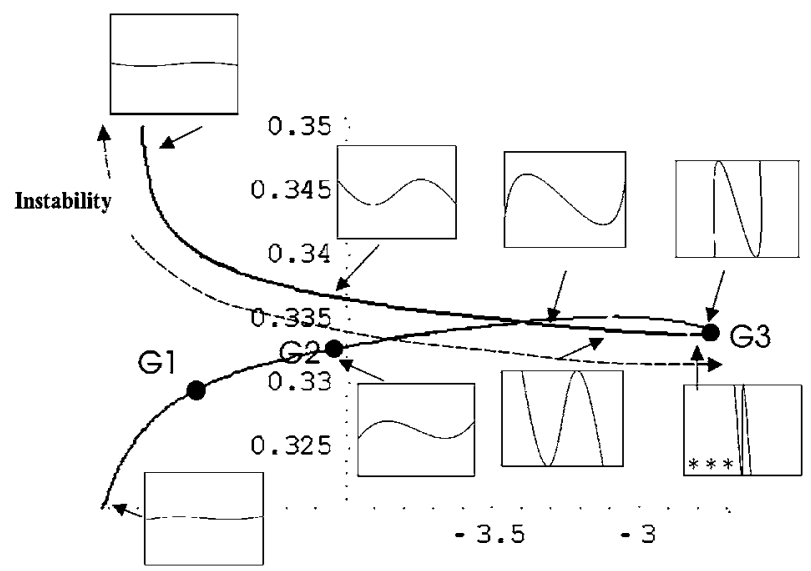

Fig. 11. Close-up of $S 13 \pm$ branch in the frequency index-logarithm of energy plane; the special periodic orbit is represented by triple stars ${ }^{(* *)}$; at certain points of the branch the corresponding motions in the configuration plane $(y, v)$ are depicted (Lee et al., 2005 [97]).

dependence of the NNMs. Different qualitative behaviours along $S 13-$ have been observed in (Lee et al., 2005 [97]):

- Near point $G_{1}$, the nonlinear attachment vibrates nearly independently and 'drives' the linear oscillator;

- in the vicinity of $G_{2}$ the oscillators vibrate as a set of 'uncoupled linear oscillators' with natural frequencies at ratio $1 / 3$, which explains why the branches $S 13 \pm$ appear as horizontal straight line segments at frequency index $1 / 3$ in the frequency-energy plot;

- as energy increases toward point $G_{3}$, because the force generated by the nonlinear spring is negligible compared to that generated by the linear spring, the linear oscillator vibrates nearly independently and drives the nonlinear attachment;

- eventually point $G_{3}$ is reached where the periodic motion is approximately given by $y(t) \approx Y \cos \omega t$, $v(t) \approx V \cos \omega t$; there occurs triple coalescence of branches $S 13 \pm$ and $S 33-$ (which is identical to $S 11-$ ).

Similar results hold for the other $S$-branches.

Periodic motions on the $U$-tongues are not NNMs because non-trivial phases between the two oscillators are realised. The motion on these tongues is represented by Lissajous curves in the configuration plane, 
whereas motion on $S$-tongues corresponds to one-dimensional curves. For example, a typical periodic orbit on branch $U 21$ is represented by a 8-shape in the configuration plane. Localisation phenomena are also detected in certain regions of $U$-tongues (Lee et al., 2005 [97]).

It turns out that specific periodic orbits (termed special orbits and represented by bullets in Fig. 9) satisfy the initial conditions $v(0)=\dot{v}(0)=y(0)=0$ and $\dot{y} \neq 0$. In addition, certain stable special orbits are localised to the nonlinear oscillator as discussed in (Lee et al., 2005 [97]). If the system initially at rest is forced impulsively, and if one of the stable, localised special orbits is excited, the major portion of the induced energy is channeled directly to the invariant manifold of that special orbit, and, hence, the motion is rapidly and passively transferred (pumped) from the linear to the nonlinear oscillator. Therefore, the impulsive excitation of one of the stable special orbits is one of the triggering mechanisms initiating (direct) passive nonlinear energy pumping in the system.

\subsubsection{Dynamics of the weakly damped system}

This section intends to demonstrate that the intricate structure of NNMs of Fig. 9 can lead to complicated transient responses of the corresponding weakly damped system. When viewed from such a perspective, one can systematically interpret the complex transitions between multi-frequency modes of the transient, weakly damped dynamics by relating them to the different branches of NNMS in the frequency-energy plot.

The response of system (2) with parameters $\omega_{0}=C=1.0, \varepsilon=0.05$ and damping coefficients $\lambda_{1}=0, \lambda_{2}=$ 0.0005 was simulated. In Fig. 12 the motion is initiated from the stable special orbit of branch $U 76$ leading to an oscillation of the system on that branch during the early stage of the motion (see Fig. 12c). As the total energy of the system decreases due to viscous dissipation there occurs a transition (jump) to the stable branch $S 12-$. On this branch, the nonlinear oscillator is locked into a transient 1:2 IR with the linear oscillator as shown in Fig. 12d; this is referred to as a 1:2 RC (Arnold, 1988 [99]; Quinn, 1997 [95]). As energy decreases even further there occurs escape from RC, and the motion evolves along branches $S 13, S 15, S 17$, etc.

The numerical evidence of these findings is given in Fig. 12e,f which depicts the dominant harmonic components of the measured displacements computed using a wavelet transform. Darkly shaded areas correspond to regions where the amplitude of the wavelet transform is high, which reveals the presence of a significant frequency component, whereas lightly shaded regions correspond to low amplitudes. A schematic presentation of these transitions in the frequency-energy plot of Fig. 9 is depicted in Fig. 13. Energy decrease due to damping dissipation triggers the transitions between different branches of NNMs, and the damped nonlinear response possesses consecutive multi-frequency stages.

The results of this example show that even low-dimensional nonlinear oscillators with relatively simple configuration may possess very complicated and rich dynamics. Clearly, the nonlinear phenomena discussed in this example cannot be realised in linear theory; it would be impossible to understand them (or even more, identify them) using linear or linearised system identification methods.

\section{Nonlinear system identification in structural dynamics: a literature review}

Nonlinear structural dynamics has been studied for a relatively long time, but the first contribution to the identification of nonlinear structural models date back to the 1970s (Ibanez, 1973 [100]; Masri and Caughey, 1979 [101]). Since then, numerous methods have been developed because of the highly individualistic nature of nonlinear systems. A large number of these methods were targeted to SDOF systems, but significant progress in the identification of multi-degree-of-freedom (MDOF) lumped parameter systems has been realised during the last 10 or 20 years. To date, simple continuous structures with localised nonlinearity are within reach. Part of the reason for this shift in emphasis is the increasing attention that this research field has attracted, especially in recent years. We note that:

- The first textbook Nonlinearity in Structural Dynamics: Detection, Identification and Modelling was written by Worden and Tomlinson (2001 [67]).

- Synthesis of nonlinear system identification in structural dynamics was made in several survey papers (Adams and Allemang, 1998 [56]; Hemez and Doebling, 2000 [102]; Worden, 2000 [57]; Hemez and Doebling, 2001a [103]). 

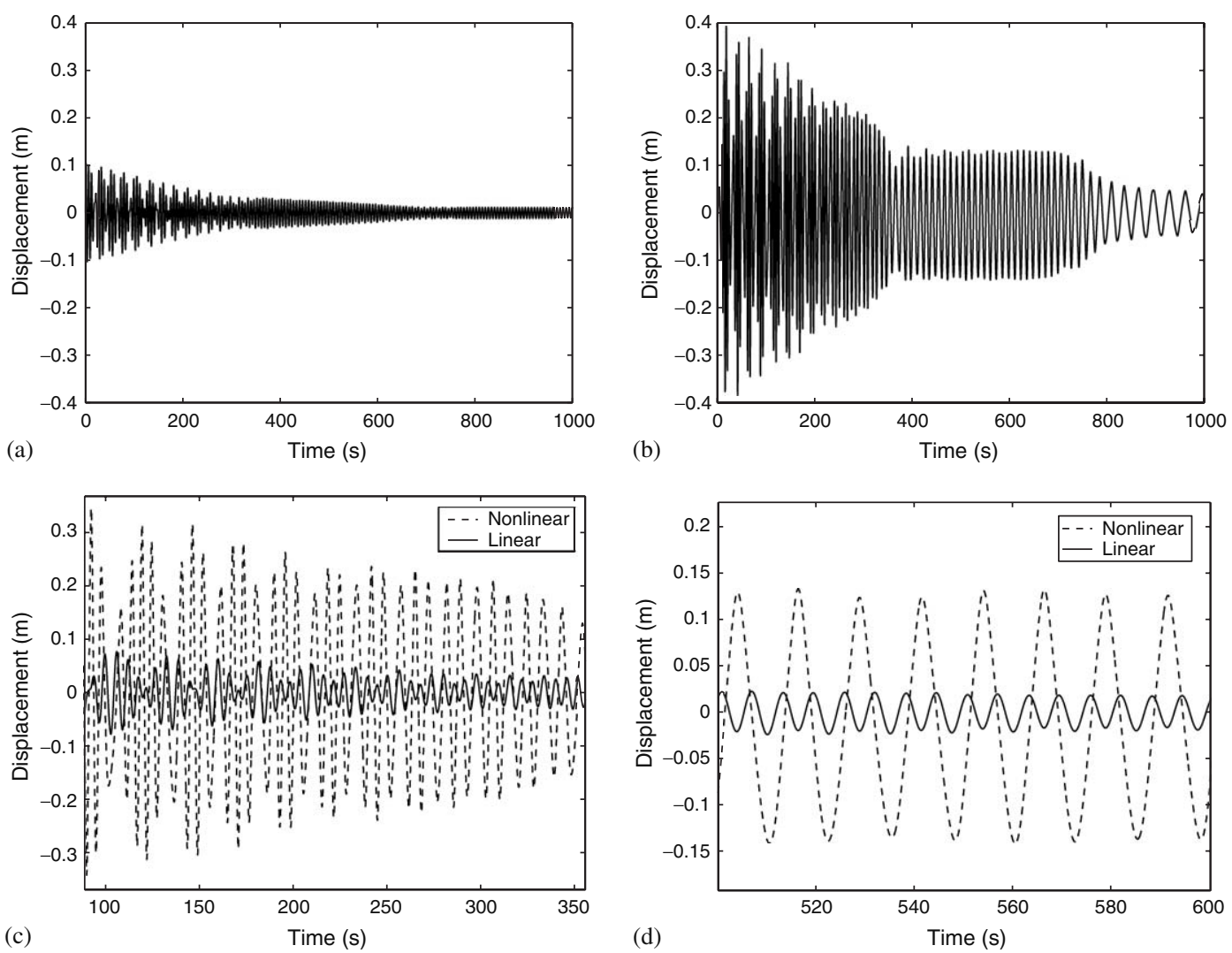

(d)

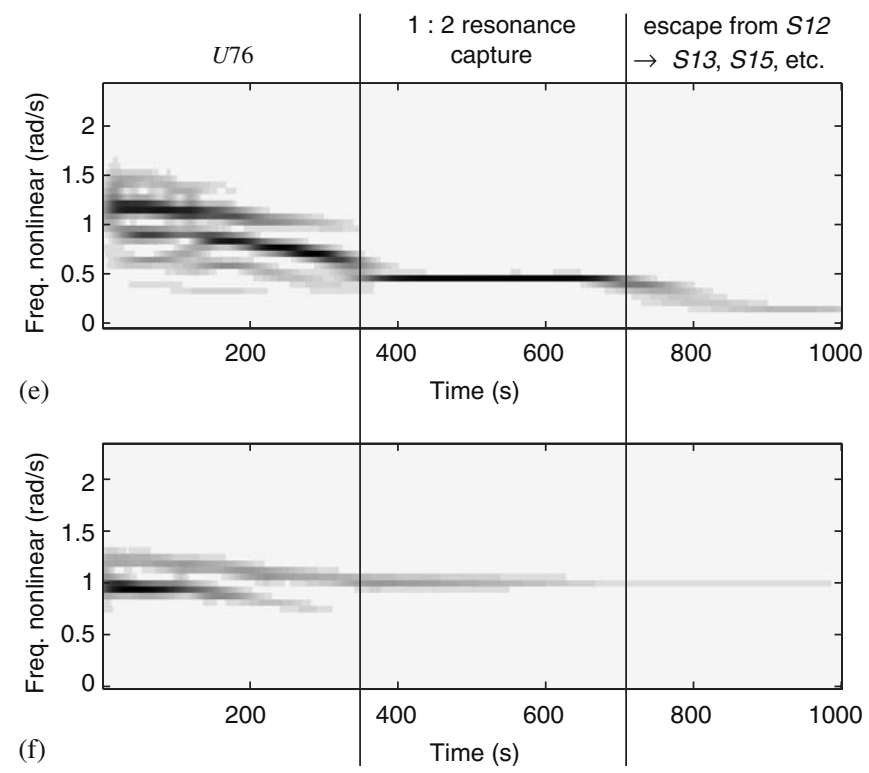

Fig. 12. Motion initiated on the stable special orbit of branch $U 76$ with weak damping: (a,b) transient responses of the linear and nonlinear oscillators; (c) early (U76), and (d) main (S12-) regimes of the motion; (e,f) wavelet transforms of the transient responses depicted in (a,b) (Kerschen et al., 2005b [53]).

- From 1997 to 2001, a working group in the framework of the European Cooperation in the field of Scientific and Technical Research (COST) Action F3 Structural Dynamics was devoted to the Identification of Nonlinear Systems (Golinval et al., 2003b [104]). Several institutions worked on two benchmarks, namely 


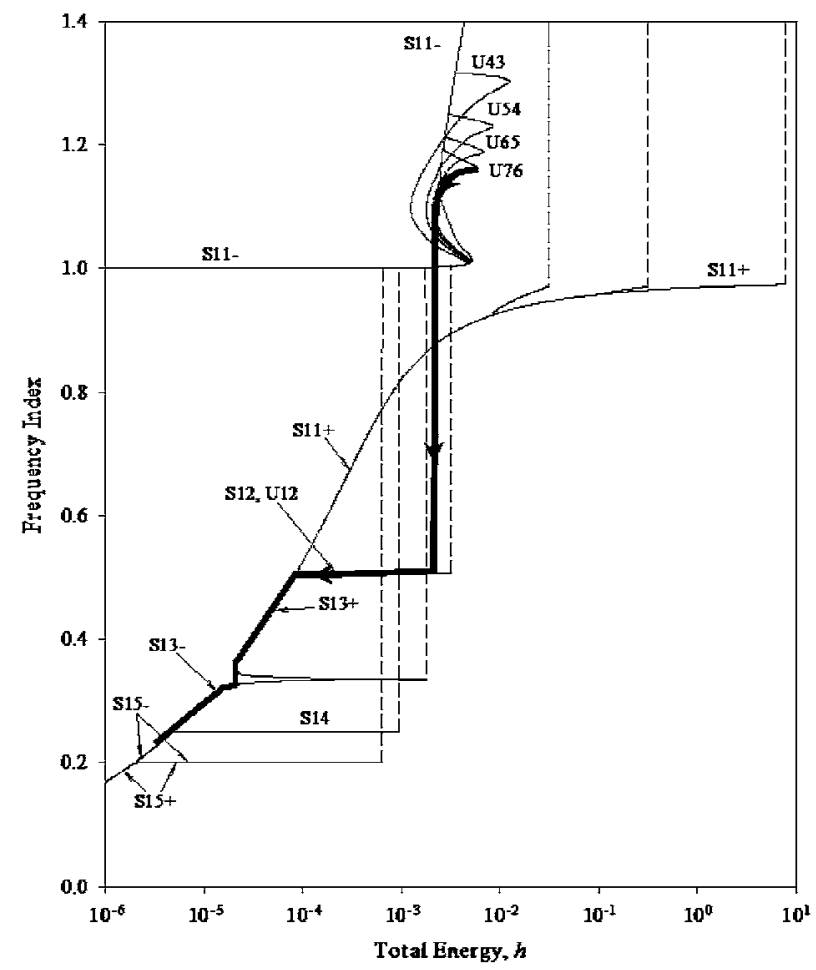

Fig. 13. Damped motion initiated on the stable special orbit of branch U76: schematic of the transitions between different branches of solutions (Lee et al., 2005 [97]).

the Ecole Centrale de Lyon (ECL) benchmark and the benchmark from the VTT Technical Research Center of Finland (see Figs. 1-2 and (Juntunen, 2003 [24]; Thouverez, 2003 [105]) for a description of the benchmarks), with different techniques.

- A special issue on Nonlinear System Identification was published in the Nonlinear Dynamics journal and edited by Dowell and Epureanu (2005 [106]).

In what follows, a review of the literature on nonlinear system identification in structural dynamics is realised. In this literature review, a relatively important number of methods are cited. For clarity, the methods are classified according to seven categories, namely by-passing nonlinearity: linearisation, time and frequencydomain methods, modal methods, time-frequency analysis, black-box modeling and structural model updating. The proposed classification is certainly not exhaustive, and additional categories could be considered, e.g., a distinction between parametric and non-parametric methods could be made. For each method, we cited the papers, we believe, which are the most relevant; these papers and the references therein should be a good point of departure for researchers and practitioners wishing to learn the theoretical foundations and the possible applications of the methods. For light reading, the authors and year of publication of a paper have been added to its reference number (the numeric style of referencing is the standard in MSSP). Each reference in the bibliography is also accompanied by the section(s) where it is cited in.

Readers who look for an introduction to nonlinear oscillations may consult (Nayfeh and Mook, 1979 [28]; Strogatz, 1994 [29]; Verhulst, 1999 [30]; Rand, 2003 [31]). More mathematically inclined readers may refer to (Guckenheimer and Holmes, 1983 [32]; Wiggins, 1990 [33]). The textbook (Worden and Tomlinson, 2001 [67]) is a reference book for anyone conducting tests on nonlinear structures and then constructing a dynamic model of the system as discussed in (Singh, 2004 [107]) (its companion is (Ewins, 2000 [11]), dedicated to linear structures). The monographs by Bendat (1998 [108]) and Bendat and Piersol (2000 [109]) are strongly recommended if random data are available. 


\subsection{By-passing nonlinearity: linearisation}

Modal analysis is an extremely powerful theory of linear systems. It is so effective in that restricted area that one might be tempted to apply the procedures of modal analysis directly to nonlinear systems without modification. In this situation, the curve-fitting algorithms used will associate a linear system with each FRFin some sense the linear system which explains it best. As the nonlinear system FRF will usually change its shape as the level of excitation is changed, any linearisation is only valid for a given excitation level. Also, because the form of the FRF is a function of the type of the excitation, different forcing types of nominally the same amplitude will require different linearisations. These are clear limitations. Linearisations based on random excitation are arguably more fundamental than those based on harmonic forcing because random excitation is the only excitation which generates nonlinear systems FRFs which 'look like' linear system FRFs.

Caughey in his seminal papers (1959 [110], 1960 [111], 1963 [34]) proposed to replace a nonlinear oscillator with external Gaussian excitation by a linear one with the same excitation such that the mean-square error between the actual nonlinear and linearised systems is minimised in a statistical sense. The procedure developed, equivalent linearisation, operated directly on the equations of motion. It is often associated in the literature with the statistical linearisation technique introduced by Kazakov (1956 [112]), but the methods are not the same as discussed in (Socha and Pawleta, 2001 [113]). Many developments have been proposed since the fundamental work of Caughey (e.g., (Iwan, 1973 [35]) and (Iwan and Mason, 1980 [114])), and a comprehensive treatment of the subject can be found in (Roberts and Spanos, 1990 [115]). This commonly used approach has proved useful in most applications, particularly for the random vibration analysis of systems where the nonlinear restoring force is hysteretic. For experimental applications, the extraction of a linear model requires the knowledge of the functional form of the restoring force, which is generally not the case. Hagedorn and Wallaschek (1987 [116]) have developed an effective experimental procedure for doing precisely this. This approach assumes that the mass matrix is known, but this limitation has been addressed by Fillatre (1992 [117]). Nonlinear system identification is carried out in (Rice, 1995 [118]) by comparing an experimentally derived equivalent linear model to the one derived directly from the assumed equation of motion.

It is well known that equivalent linearisation does not correctly predict the response of strongly nonlinear random oscillators. An interesting generalisation of this technique was proposed by Miles (1989 [119]). The approach involves calculating the expected value of the spectral response of an equivalent linear system where the equivalent natural frequency is assumed to be a random variable. ${ }^{3}$ This work triggered the development of the concept of equivalent linear systems with random coefficients (Bouc, 1994 [121]; Soize, 1994 [122]) which has enjoyed some success for system identification of nonlinear systems (Soize, 1995 [123]; Soize and Le Fur, 1997 [124]; Bellizzi et al., 1998 [125]; Bellizzi and Defilippi, 2003 [126]).

The harmonic balance method described in (Nayfeh and Mook, 1979 [28]) can be employed for linearising nonlinear equations of motion with harmonic forcing. By considering one dominant harmonic in the analysis (i.e., by assuming that sub- and superharmonics are negligible compared to the fundamental harmonic), it offers a means of approximating the FRFs of nonlinear systems (in this case, the harmonic balance method is often referred to as the describing function method). This method has been the basis of several nonlinear system identification techniques (see, e.g., (Yasuda et al., 1988a,b [127,128]; Benhafsi et al., 1992 [129]; Meyer et al., 2003 [130]; Ozer et al., 2005 [131])).

\subsection{Time-domain methods}

A method is said to be a time-domain method if the data considered during the identification process take the form of time series (e.g., force and acceleration). Such techniques have the advantage that the signals are directly provided by current measurement devices; less time and effort is spent on data acquisition and processing.

One fruitful approach to time-domain identification, the restoring force surface (RFS) method, began with Masri and Caughey's 1979 paper [101]; a parallel approach named force-state mapping was developed

\footnotetext{
${ }^{3}$ We note that the concept of random natural frequency already appeared in (Crandall, 1963 [120]).
} 
independently in (Crawley and O’Donnell, 1986 [132]; Crawley and Aubert, 1986 [133]). The RFS method which is described in Section 6.1 initiated the analysis of nonlinear structural systems in terms of their internal RFSs. It was first developed for SDOF systems, but the generalisation to MDOF systems soon followed (Masri et al., 1982 [134]). The method was extremely appealing in its simplicity, the starting point being Newton's second law. However, the initial version depended on the rather arbitrary use of Chebyshev polynomials for the expansion of the nonlinear restoring forces. This made for a rather complicated numerical analysis. The approach also suffered from bias unless the identification was iterated, and this made it rather time-consuming. A further signal processing demand was that displacement, velocity and acceleration data were required for each DOF necessitating the use of complex instrumentation or further numerical analysis. All these exceptions were overcome in the following years by an array of researchers (Yang and Ibrahim, 1985 [135]; Masri et al., 1987a,b [136,137]; Al-Hadid and Wright, 1989, 1990, 1992 [138-140]; Worden, 1990a,b [141,142]; Mohammad et al., 1991 [143]; Shin and Hammond, 1998a [144]); the application of the method in the frequency domain was also proposed in (Kim and Park, 1994 [145]). Extension of the technique to cover hysteretic and chaotic systems have been devised (Lo and Hammond, 1988 [146]; Benedettini et al., 1991 [147]; Shin and Hammond, 1998b [148]). The method has proved to be a robust addition to the structural dynamicists toolbox, and various experimental studies have been published, notably for the identification of nonlinear automotive components (Audenino et al., 1990 [149]; Belingardi and Campanile, 1990 [150]; Surace et al., 1992 [151]; Cafferty et al., 1993 [152]; Duym et al., 1996a [153]). The latter reference sparked a resurgence of interest in the theory of the method (Duym et al., 1995 [66], 1996b,c [154,155]). The RFS method still enjoys some recent applications, adaptations and developments. For instance, experimental investigations are performed in (Kerschen et al., 2001a [156]; Meskell et al., 2001 [157]), curve-fitting is avoided in (Dimitriadis and Cooper, 1998 [158]) and nonlinear system identification in the absence of input measurement is proposed in (Haroon et al., 2004, $2005[159,160]$ ).

A technique which was widely applied in Control Engineering at first, but was taken up by structural dynamicists, was time-series analysis. The linear variant of the approach based on ARMA (Auto-Regressive Moving Average) models has long been used for modeling and prediction purposes (Box and Jenkins, 1970 [161]). There have been numerous attempts to generalise the model structure to the nonlinear case, arguably the most versatile and enduring structure has been the NARMAX (Nonlinear ARMA with eXogeneous input) model proposed by Leontaritis and Billings (1985a,b [162,163]). A detailed discussion of this method is given in Section 6.3. Since the inception of the method, there have been many developments, notably the introduction of an orthogonal estimation algorithm (Korenberg et al., 1988 [164]), which allows model parameters to be estimated sequentially so that the complexity of the model can be controlled. Also noteworthy are the correlation tests designed to assess model validity (Billings et al., 1989c [165]). The NARMAX structure is general enough to admit many forms of model including neural networks although the estimation problem becomes nonlinear and the orthogonal estimator will not work (Billings et al., 1991a [166]). If a radial basis function network structure is used, however, under certain training conditions, the linear-in-the-parameters estimation problem is recovered (Chen et al., 1990a [167]). The NARMAX theory also makes a very useful contact with the functional series methods. Using a variant of the harmonic probing algorithm (Bedrosian and Rice, 1971 [168]), it is possible to extract the HOFRFs directly from the NARMAX model (Billings and Tsang, 1989a,b [169,170]). The input and output variables are usually physical quantities like force and displacement response, respectively. An interesting alternative approach to this was followed by Thouverez and Jezequel (1996 [171]), who fitted a NARMAX model using modal coordinates.

Most applications of the Hilbert transform (e.g, nonlinearity detection) used the frequency-domain Hilbert transform. In contrast, Feldman showed how to use the traditional definition of the analytic signal and the time-domain Hilbert transform in order to identify nonlinear models of SDOF systems. The FREEVIB approach proposed in (Feldman, 1994a [172]) is based on free vibration whereas the FORCEVIB approach proposed in (Feldman, 1994b [173]) deals with forced vibration. As explained in Section 6.4, these approaches can be used to construct the nonlinear damping and stiffness curves for a large class of nonlinear systems, but are only suitable for monocomponent signals. A practical application to a nonlinear ocean mooring system is detailed in (Gottlieb et al., 1996 [174]), and the extension to two-component signals is discussed in (Feldman, 1997 [175]). We mention that a method for the decomposition of signals with multiple components into a collection of monocomponents signals, termed intrinsic mode functions (IMFs), was proposed in Huang et al. 
(1998 [176]) and is now referred to as Huang-Hilbert transform in the technical literature. The IMFs are constructed such that they have the same number of extrema and zero-crossings, and only one extremum between successive zero-crossings. As a result, they admit a well-behaved Hilbert transform. The method now enjoys several applications in structural dynamics including linear system identification (Yang et al., 2003a,b $[177,178]$ ) and damage detection (Yang and Lin, 2004b [179]).

Other time-domain techniques have been proposed. They include a nonlinear version of the stochastic subspace identification approach (Lacy and Bernstein, 2001 [180]), a method based upon the Lie series solutions (Pilipchuk and Tan, 2005 [181]), methods for the simultaneous estimation of Coulomb and viscous friction (Feeny and Liang, 1996 [182]; Liang and Feeny, 1998, 2004a [183,184]; Singh et al., 2001 [185]), an observer-based method (Chatterjee and Cusumano, 2003 [186]) and two techniques for identification of nonlinear beams (Yasuda and Kamiya, 1999 [187]).

\subsection{Frequency-domain methods}

A method is said to be a frequency-domain method if the data considered during the identification process take the form of FRFs or spectra. A wide range of frequency-domain methods have been proposed in the technical literature during the last two decades.

An early attempt to exploit frequency-domain data for the purpose of nonlinear system identification was through the use of functional series-the Volterra and Wiener series (A comprehensive description on the Volterra and Wiener theories of nonlinear systems is given by Schetzen (1980 [188])). These series enable the generalisation of the concept of impulse response function and FRF to nonlinear systems, as discussed in Section 6.5. HOFRFs have enjoyed greater popularity, at least for the purpose of system identification (see (Thouverez and Jezequel, 1998 [189]) for a time-domain analysis), perhaps because frequency-domain treatment offers easier computation and more intuitive interpretation. The first major application in the field of structural dynamics occurred with the work of Gifford (1989 [190]). He proposed to extract nonlinear parameters by fitting surfaces or hypersurfaces to the HOFRFs. This work was extended by Storer (Storer, 1991 [191]; Storer and Tomlinson, 1993 [192]), who demonstrated that it is sufficient to curve-fit to the parts of the FRFs above the diagonal frequency subspaces. Parameter estimation of SDOF systems using HOFRFs was also investigated in (Khan and Vyas, 1999 [193]; Chatterjee and Vyas, 2003 [194]), MDOF oscillators being studied in (Khan and Vyas, 2001b [195]; Chatterjee and Vyas, 2004 [196]). The experimental identification of a rotor-bearing system was reported in (Khan and Vyas, 2001a [197]). The extension of modal analysis to nonlinear structures was discussed in (Tawfiq and Vinh, 2003, 2004 [198,199]).

Higher-order spectra have also received some consideration for system identification (Bendat, 1998 [108]). Realising that only the equivalent linear damping level can be estimated using second-order spectra (Roberts et al., 1995 [200]), and Vasta and Roberts (1998 [201]) developed a procedure involving the fourth-order spectrum, namely the trispectrum. In addition, the excitation measurement is not necessary; it is possible, in principle, at least, to formulate non-Gaussian models of the excitation in a parametric form and to estimate the parameters in such models, alongside various system parameters (Roberts and Vasta, 2000a [202]). To overcome the assumption of known parametric form for the input spectra, an energy identification technique was also proposed in (Roberts and Vasta, 2000b [203]). In (Hajj et al., 2000 [204]), multiple scales and the third-order spectrum, namely the bispectrum, are combined to characterise and quantify damping and nonlinear parameters of the first mode of a three-beam two-mass frame. An extensive bibliography on higherorder statistics can be found in (Swami et al., 1997 [205]).

Other early contributions to nonlinear system identification using frequency-domain data are those of Yasuda and co-authors $(1988 \mathrm{a}, \mathrm{b}[127,128])$ in which the harmonic balance method is used in an inverse way to estimate parameters. Elastic structures were also investigated in (Yasuda and Kamiya, 1990, 1997 [206,207]). These studies targeted nonlinear systems with periodic steady state response resulting from external excitation. In (Yuan and Feeny, 1998 [208]), the harmonic balance-based identification was extended to nonlinear systems with chaotic behaviour; an experimental magnetoelastic oscillator was investigated in (Feeny et al., 2001 [209]) and a MDOF system in (Liang and Feeny, 2004b [210]). Harmonic balance-based methods were also considered in (Thothadrai et al., 2003 [211]; Thothadrai and Moon, 2005 [212]) for identification of fluid-structure systems with self-excited motions. 
Spectral methods based on the reverse path analysis were developed and utilised for identification of SDOF nonlinear systems in (Rice and Fitzpatrick, 1988 [213]; Esmonde et al., 1990a,b [214,215]; Bendat, 1990 [216]; Rice and Fitzpatrick, 1991a [217]; Bendat et al., 1995 [218]; Zeldin and Spanos, 1998 [219]). The concept of reverse path is discussed at length in (Bendat, 1998 [108]), and for its historical evolution, the reader may refer to the extensive literature review provided by Bendat (1993 [220]). A generalisation of reverse path spectral methods for identification of MDOF systems was first proposed in (Rice and Fitzpatrick, 1991b [221]). This method determines the nonlinear coefficients together with a physical model of the underlying linear structure and requires excitation signals at each response location. A second alternative referred to as the conditioned reverse path (CRP) method was presented in (Richards and Singh, 1998 [222]) and is exposed in Section 7.1. It estimates the nonlinear coefficients together with a FRF-based model of the underlying linear structure and does not ask for a particular excitation pattern (we note that a physical model of the underlying structure can also be built using structural model updating techniques as discussed in (Kerschen and Golinval, 2005a [223])). A detailed discussion of the fundamental differences between the two techniques is given in (Fitzpatrick and Rice, 2000 [224]; Richards and Singh, 2000a,b [225,226]). The CRP method was compared to the RFS method using numerical examples in (Richards and Singh, 1999 [227]) whereas it was used for identification of experimental systems in (Kerschen et al., 2001b, 2003a [228,229]; Garibaldi, 2003 [230]; Marchesiello, 2003 [231]).

The nonlinear identification through feedback of the output (NIFO) method presented in Section 7.2 is another interesting spectral approach which was proposed by Adams and Allemang (2000a [232]). The central idea of the method is to treat the nonlinear forces as internal feedback forces in the underlying linear model of the system as discussed in (Adams and Allemang, 1999a,b [233,234]). By coupling the NIFO and RFS methods, an algorithm for nonlinear system identification in the absence of input measurements was also developed in (Haroon et al., 2005 [160]).

Other frequency-domain approaches include system identification using associated linear equations (Vazquez Feijoo et al., 2004 [235]), methods for parameter estimation of squeeze film dampers (Zhang and Roberts, 1996 [236]), methods based upon curve-fitting experimental frequency- and force-response data points (Krauss and Nayfeh, 1999 [237]; Malatkar and Nayfeh, 2003a [238]) and methods exploiting nonlinear resonances (Nayfeh, 1985 [239]; Fahey and Nayfeh, 1998 [240]).

Finally, comparison between time- and frequency-domain algorithms can for instance be found in (Richards and Singh, 1999 [227]; Doughty et al., 2002 [241]) and in the special issue dedicated to the COST Action F3 Structural Dynamics in the Mechanical Systems and Signal Processing journal (Golinval and Link; 2003a [242]).

\subsection{Modal methods}

Modal analysis is indubitably the most popular approach to performing linear system identification in structural dynamics (Heylen et al., 1997 [9]; Maia and Silva, 1997 [10]; Ewins, 2000 [11]). The model of the system is known to be in the form of modal parameters, namely the natural frequencies, mode shapes and damping ratios. The popularity of modal analysis stems from its great generality; modal parameters can describe the behaviour of a system for any input type and any range of the input.

Traditional techniques for analysing the dynamics of nonlinear structures are based on the assumptions of weak nonlinearities and of a 'nonlinear' modal structure that is similar or a small perturbation of the underlying linearised system (see, e.g., (Piranda et al., 1998 [243])). Although this may be the case for some aerospace structures as commented in (Göge et al., 2004 [244]), the discussion in Section 2.1 shows that this approach to nonlinear system identification may lead to erroneous results when mode bifurcations are responsible for additional, essentially nonlinear modes of vibration.

Thanks to the seminal work of Rosenberg (1962, 1966 [36,37]), the concept of NNM was introduced and further developed in (Rand, 1974 [38]; Shaw and Pierre, 1993 [39]; Vakakis et al., 1996 [40]). The focus in this section is on the inverse problem, i.e., on the development of a nonlinear model in modal space from experimental measurements. For a discussion of NNMs, the reader may refer to Section 2 and to the monograph (Vakakis et al., 1996 [40]), and should consult (Vakakis, 1997 [41]) for their applications in vibration theory. 
The NNMs provide a rigorous theoretical framework for extending modal analysis to nonlinear systems. It is therefore attractive to develop nonlinear system identification techniques based on these nonlinear modes. However, in contrast to the linear theory, NNMs and their period are amplitude-dependent, which complicates the analysis. Szemplinska-Stupnicka (1979, 1983 [245,246]) showed that the mode of vibration in resonant conditions can be considered as a good approximation of the NNM. The stationary solution can then be expanded in terms of the NNM and its corresponding natural frequency, both of which are function of the modal amplitude. The numerical computation of these modal parameters involves a nonlinear eigenvalue problem generally solved using a Newton-Raphson procedure. By curve-fitting forced responses in the neighbourhood of a resonance, the nonlinear modal parameters can be obtained from experimental data.

Based on this methodology, modal parameter identification procedures for forced response of nonlinear systems were developed in (Jezequel, 1987 [247]; Setio et al., 1992a,b [248,249]; Chong and Imregun, 2001 [250]). In these studies, it is assumed that the resonant frequencies are not close to each other, and the frequency response is expressed as a linear combination of contributions from resonant NNMs. The coupled nature of the modal space is accounted for, by adding (small) contributions from the non-resonant modes. The modal parameters of the resonant NNMs behave nonlinearly with the modal amplitudes, and, as explained above, they are identified by curve-fitting procedures. The non-resonant modal parameters are merely the linear modal parameters which are computed by standard modal analysis at sufficiently smallamplitude motions. In (Gibert et al., 1999 [251]), the procedure was applied to an aircraft landing gear, whereas the ECL benchmark depicted in Fig. 1 was analysed in (Gibert, 2003 [252]). Identification of amplitude-dependent nonlinear modes using successive approximation model is proposed in (Huang and Iwan, 1997 [253]).

The identification of individual NNMs may represent a limitation when considering the arbitrary motion of a nonlinear system; in this case, the NNMs are bound to interact. To progress in this direction, Pesheck et al. (2001a [254]) have introduced the notion of multi-mode invariant manifold which takes the possible modal interactions into account. To our knowledge, this concept has not yet been exploited for nonlinear system identification, but it should form the basis of future methods.

The method proposed by Wright et al. (2001 [255]) targets a multi-stage identification of large structures with high modal density and weakly nonlinear effects (see Section 7.3). It is based on a linear modal space model in which the modes may be coupled nonlinearly by additional terms in the equations of motion. The key idea is to reduce the scale of the identification problem by classifying the modes into different categories (i.e., influenced or not by nonlinear effects, coupled or uncoupled in damping and/or nonlinearity) and by extending the classical linear force appropriation approach (Williams et al., 1986 [256]; Wright et al., 1999 [257]) to non-proportionally damped nonlinear systems. For this purpose, two methodologies which enable the treatment of modes individually or in small groups were developed: the force appropriation of nonlinear systems (FANS, (Atkins et al., 2000 [258])) method and the nonlinear resonant decay method (NLRD, Wright et al. (2001 [255])). A 'low-order' regression analysis in modal space is then carried out using the classical RFS method (Masri et al., 1982 [134]; Al-Hadid and Wright, 1989 [138]). Experimental validation of the method using a single-bay panel structure and a wing-store structure is available in (Platten et al., 2002 [259]) and in (Platten et al., 2004 [260]), respectively.

Finally, several authors have used 'other nonlinear modes' for identification of nonlinear systems from free vibration. In (Bellizzi et al., 2001 [261]), identification is performed by comparing experimental coupled nonlinear modes to the predicted ones. In (Hasselman et al., 1998 [262]; Hemez and Doebling, 2001a [103]; Lenaerts et al., 2001, 2003 [263,264]), a similar procedure is derived for the modes extracted from the POD, termed proper orthogonal modes (POMs). In (Argoul et al., 2004 [265]), instantaneous mode shapes are extracted using the wavelet transform. All these modes do not have the theoretical foundations of the NNMs, but they provide an appealing alternative, at least for nonlinear system identification, because (i) they are sensitive to the nonlinear behaviour; (ii) their computation is relatively straightforward (e.g, the POMs are readily obtained from a singular value decomposition of the response matrix) and (iii) they obey to 'a sort of principle of superposition' due to the fact that the original signal is retrieved when all the modal contributions are added up. 


\subsection{Time-frequency analysis}

A typical feature of nonlinear vibrations is that the instantaneous natural frequency and damping coefficient of the system may become functions of time depending upon the type of nonlinearity. One possible means of studying the time-varying nature of the system vibration characteristics is to compute backbone and damping curves, an elegant method for their computation being the time-domain version of the Hilbert transform. However, one difficulty of this approach is in the case of multicomponent signals as already discussed in Section 3.2.

A variety of methods for time-frequency analysis of non-stationary signals have been proposed in the literature. Time-frequency methods do not provide additional insight into the system dynamics compared with combined time- and frequency-domain analyses, but, due to their specific representation, they offer a different perspective of the dynamics. Another advantage of time-frequency decompositions is that they permit projections of the time history onto a space that allows separation of components of the signal, which facilitates filtering in the case of multicomponent signals. This class of methods was already considered in the 1960s for problems in acoustics and vibrations (Priestley, 1967 [266]; Hammond, 1968 [267]), but it is only from the 1990s that it gained widespread popularity within the structural dynamics community. A survey of the analysis of non-stationary signals using time-frequency methods is available in (Hammond and White, 1996 [268]).

Linear systems have been investigated using this class of methods, mainly for the purpose of natural frequency and damping estimation (see, e.g., (Park et al., 1995 [269]; Ruzzene et al., 1997 [270]; Staszewski, 1997 [271]; Argoul and Le, 2004 [272]; Boltezar and Slavic, 2004 [273])).

Time-frequency analysis is also suitable for the analysis of nonlinear oscillations. Linear representations which decompose a signal in terms of a set of simpler components which when added up yield the original signal have been used in nonlinear structural dynamics. For instance, nonlinear oscillations are studied using the Gabor transform in (Spina et al., 1996 [274]; Franco and Pauletti, 1997 [275]). An overview of the use of the wavelet transform in nonlinear dynamics can be found in (Staszewski, 2000 [276]). Quadratic representations which include the Wigner-Ville distribution and the Cohen-class of distributions have also received some attention (Feldman and Braun, 1995 [277]; Wang et al., 2003a [278]). Focusing now on the inverse problem, i.e., on the extraction of parameters from measured data, the joint application of the Gabor and Hilbert transforms is proposed in (Spina et al., 1996 [274]). The Gabor transform identifies a time-variant matrix representing the spatial behaviour of the system; this matrix is used to decouple the transient response into a set of quasi-harmonic components. The Hilbert transform is then applied to identify the dissipative and elastic restoring forces associated with each component. In (Bellizzi et al., 2001 [261]), the Gabor transform is used for the computation of coupled nonlinear modes from measured data, nonlinear modal parameters being extracted from these modes. In (Staszewski, 1998 [279]), the backbone curve and the signal envelope are extracted based on the ridges and skeletons of the wavelet transform, which enables parameter estimation of SDOF and MDOF nonlinear systems. Wavelet-based identification is also performed in (Garibaldi et al., 1998 [280]; Argoul and Le, 2003 [281]). A comparison between the RFS method and the approach proposed in (Garibaldi et al., 1998 [280]) is available in (Lenaerts et al., 2004 [282]). We note that all these methodologies deal with free oscillations.

A different approach using the wavelet transform for nonlinear system identification consists in expanding the system response and excitation in terms of scaling functions (in (Kitada, 1998 [283])), it is the tangent stiffness of the structural system which is expressed as a series expansion of wavelets). This wavelet-Galerkin oriented procedure transforms the original nonlinear differential problem into a set of algebraic equations in the unknown parameters and has been implemented for free and forced vibrations using the Daubechies' scaling functions (Ghanem and Romeo, 2001 [284]). A variant of this method using the Haar wavelet is proposed in (Chen and Ho, 2004 [285]).

Alternative approaches for identification of nonlinear parameters are those employing the Wigner-Ville distribution (Feldman and Braun, 1995 [277]) or a quadratic time-frequency distribution of Cohen class (Wang et al., 2003b [286]). 


\subsection{Black-box modeling}

One major difficulty of nonlinear system identification is that the functional $S[\bullet]$ which maps the input $x(t)$ to the output $y(t), y(t)=S[x(t)]$, is generally unknown beforehand. Physical insight is most often of great help to select a reasonably accurate model of the nonlinearity. Only if this gives unsatisfactory results or if physical insight is completely lacking, it is then time to move to nonlinear black-box modeling. A nonlinear black-box structure is a model structure that is prepared to describe virtually any nonlinear dynamics on the basis of the data alone. This is a very ambitious objective knowing that nonlinearity may be caused by many different mechanisms and may result in plethora of dynamic phenomena, but there exist theorems supporting this finding (e.g., Cybenko (1989 [287]) showed that neural networks are universal approximators).

As extensively discussed in (Sjöberg et al., 1995 [288]), nonlinear black-box modeling is as a mapping from past observed data to a regressor space followed by a nonlinear, function expansion type, mapping to the space of the system's outputs. Some methods for performing the nonlinear mapping are:

- artificial neural networks have come into prominence because of their universal approximation features;

- wavelet networks are attractive because they unify multi-resolution features of wavelet bases and universal approximation features of neural networks;

- splines are interesting functions, because they are computationally very simple, can be made as smooth as desired and are very economic to store;

- neuro-fuzzy models combine the semantic transparency of rule-based fuzzy systems with the learning capabilities of neural networks; they can be regarded more as grey-box models.

Most of the possibilities for black-box nonlinear dynamical models are described in a common framework in two excellent papers: (Juditksy et al., 1995 [289]) is geared toward the theoretical aspects, and (Sjöberg et al., 1995 [288]) focuses on the user and algorithmic aspects.

Some of the earliest examples of the use of neural networks for system identification and modeling are the work of Chu et al. (1990 [290]) and Narendra and Parthasarathy (1990 [291]). The most comprehensive programme of work to date is that of Billings and co-workers starting with (Billings et al., 1991a [166]) for the multi-layer perceptron structure and (Chen et al., 1990b [292]) for the radial basis function network. Masri et al. $(1992,1993$ [293,294]) were amongst the first structural dynamicists to exploit the techniques. The latter work is interesting because it demonstrates 'dynamic neurons' which are said to increase the utility of the multi-layer perceptron structure. In (Worden et al., 1994a,b [295,296]), it is shown that multi-layer perceptron neural networks can represent a broad range of SDOF nonlinear systems, with continuous or discontinuous nonlinearities. In (Chassiakos and Masri, 1996 [297]), the identification of MDOF systems is considered. Smyth and co-workers (Kosmatopoulos et al., 2001 [298]; Pei et al., 2004 [299]) have developed a procedure for adaptive identification of nonlinear hysteretic dynamic systems by using Volterra-Wiener neural networks which combine a dynamic linear module in series with a static neural network module. In (Le Riche et al., 2001 [300]; Song et al., 2004 [301]), the neural network does not learn the functional which relates the system's input $x(t)$ to its output $y(t)$ but rather the relation that links carefully selected structural features and the parameters to be identified. Fuzzy adaptive neural networks are investigated in (Liang et al., 2001 [302]) to increase the training speed of the network. In (Fan and Li, 2002 [303]), a hybrid approach that embeds neural networks to represent unknown nonlinearities in a otherwise typical physical model is developed. A similar study employing radial basis function network is proposed in (Saadat et al., 2004 [304]).

Clearly, among the different choices for nonlinear black-box modeling, artificial neural networks have received the most attention in nonlinear structural dynamics. The use of splines for non-parametric identification of wire rope isolators is investigated in (Peifer et al., 2003 [305]). Neuro-fuzzy models and wavelet networks can also be useful for nonlinear system identification (Liu et al., 2000 [306]; Babuska and Verbruggen, 2003 [307]), but they have not yet been applied extensively for this purpose in structural dynamics.

We conclude this section by noting that black-box modeling has also some limitations. First and foremost, the identified model parameters do not provide physical information about the structure, which may limit the practical usefulness of the resulting model. An important question is how to deal with the large number of 
potentially necessary parameters required to handle arbitrary nonlinear dynamical systems, which may lead to overfitting (the trade-off between bias and variance must therefore be adequately addressed) and may imply a high-dimensional nonlinear optimisation problem for the estimation of the parameters.

\subsection{Structural model updating}

For the investigation of more complex structures in a wider frequency range, resorting to models with many DOFs is inevitable. However, the estimation of all the model parameters from experimental measurements may quickly become intractable. A solution to this problem is to use structural modeling techniques which compute the model parameters based on the knowledge of the geometrical and mechanical properties of the structure.

Despite the high sophistication of structural modeling, practical applications often reveal considerable discrepancies between the model predictions and experimental results, due to three sources of errors, namely modeling errors (e.g, imperfect boundary conditions or assumption of proportional damping), parameter errors (e.g., inaccuracy of Young's modulus) and testing errors (e.g, noise during the measurement process). There is thus the need to improve structural models through the comparison with vibration measurements performed on the real structure; this is referred to as structural model updating.

Very often, the initial model is created using the finite element method (see, e.g., (Zienkiewicz, 1977 [308])), and structural model updating is termed finite element model updating. Finite element model updating was first introduced in the 1970s for linear structures (Berman and Nagy, 1971 [309]; Baruch, 1978 [310]). For a detailed description of this field of research and the issues commonly encountered (e.g., model matching step and error localisation), the reader is invited to consult (Natke, 1992 [311]; Friswell and Mottershead, 1995 [312]; Link, 1999 [313]).

The literature on methods that propose to update nonlinear dynamic models is rather sparse. In (Schmidt, 1994 [314]), parameters of nonlinear elements are updated by fitting simulated time history functions and the corresponding measurement data. The problem of estimating the initial values as well as the problem of increasing error between simulated and measured time history functions is overcome by using the method of modal state observers. Kapania and Park (1997 [315]) proposed to compute the sensitivity of the transient response with respect to the design parameters using the time finite element method. The minimum model error estimation algorithm is exploited in (Dippery and Smith, 1998 [316]) to produce accurate models of nonlinear systems. In this algorithm, a two-point BVP is solved in order to obtain estimates of the optimal trajectories together with the model error. In (Kyprianou, 1999 [317]; Kyprianou et al., 2001 [318]), model updating is realised through the minimisation of an objective function based on the difference between the measured and predicted time series. The optimisation is achieved using the differential evolution algorithm which belongs to the class of genetic algorithms. The formulation proposed by Meyer and co-authors (Meyer and Link, 2002 [319]; Meyer et al., 2003 [130]) involves a linearisation of the nonlinear equilibrium equations of the structure using the harmonic balance method. Updating of the finite element model is carried out by minimising the deviations between measured and predicted displacement responses in the frequency domain. In (Yuen and Beck, 2003 [320]), model updating is performed in the presence of incomplete noisy response measurements. A stochastic model is used for the uncertain input, and a Bayesian probabilistic approach is used to quantify the uncertainties in the model parameters. In (Kerschen and Golinval, 2005a [223]), a two-step methodology which decouples the estimation of the linear and nonlinear parameters of the finite element model is proposed. This methodology takes advantage of the CRP method and is applied to a numerical application consisting of an aeroplane-like structure.

Due to the inapplicability of modal analysis, test-analysis correlation which is inherent to structural model updating is a difficult task in the presence of nonlinearity. Several efforts have been made in order to define features (i.e., variables or quantities identified from the structural response that give useful insight into the dynamics of interest) that facilitate correlation. In the case of pyroshock response, NASA has proposed criteria such as peak amplitude, temporal moments and shock response spectra as appropriate features of the response signal (Mulville, 1999 [321]). In (Doebling et al., 2000 [322]; Schultze et al., 2001 [17]), the peak response and time of arrival are defined as features in order to study the transient dynamics of a viscoelastic material. In (Song et al., 2004 [301]), the envelope of transient acceleration responses is considered as the best 
information to identify joint parameters associated with adjusted Iwan beam elements. The POD method, also known as Karhunen-Loève transform or principal component analysis, has been investigated in several studies (Ma and Vakakis, 1999 [323]; Hasselman et al., 1998 [262]; Hemez and Doebling, 2001a [103]). Specifically, the modes extracted from the decomposition, the POMs, have been shown to be interesting features for the purpose of test-analysis correlation. In (Lenaerts et al., 2001, 2003 [263,264]), the POMs together with the wavelet transform of their amplitude modulations are considered for finite element model updating. Although it is frequently applied to nonlinear problems, it should be borne in mind that the POD only gives the optimal approximating linear manifold in the configuration space represented by the data. This is the reason why finite element model updating was performed in (Kerschen, 2003c [324]; Kerschen and Golinval, 2004a [325]) using the features extracted from a nonlinear generalisation of the POD, termed nonlinear principal component analysis (Kramer, 1991 [326]). In (Zimmerman et al., 2005 [327]), the POD is coupled with neural network and genetic algorithms for approximation and calibration of nonlinear structural models.

A statistics-based model updating and validation philosophy is proposed in (Doebling et al., 2000 [322]; Schultze et al., 2001 [17]). The motivation for including statistical analysis is driven by the desire to account for the effects of environmental and experimental variability. The feature comparison is implemented using metrics such as Mahalanobis distance and Kullback-Leibler relative entropy function. In addition, the finite element model is replaced by an equivalent meta-model with a much smaller analytical form. This strategy aims at reducing the number of computer simulations required during optimisation while maintaining the pertinent characteristics of the problem. The demonstration application consists in analysing the response of a steel/polymer foam assembly during a drop test.

As stated in the introductory section, model updating, being a particular class of system identification techniques, is an integral part of the verification and validation process. We note that the first book dedicated to this subject in structural dynamics has been recently published (Hemez et al., 2005 [64]).

\section{Detection of nonlinearity}

\subsection{Literature review}

According to the scheme in Fig. 4, the detection of structural nonlinearity is the first step toward establishing a structural model with a good predictive accuracy. Since the focus in this overview paper is mainly on parameter estimation techniques, the relevant methods for nonlinearity detection will only be cited or briefly described. More details can be found in the references cited throughout this section. An excellentthough not up to date-overview is available in (Wyckaert, 1992 [328]). More recent overviews can be found in (Adams and Allemang, 1998 [56]; Vanhoenacker et al., 2002 [329]; Gloth and Göge, 2004 [330]). A number of different approaches are also described in (Ewins, 2000 [11]; Worden and Tomlinson, 2001 [67]; Wong et al., 2002 [331]).

As discussed in Section 2.1, various concepts and analytical constructions for the analysis of linear systems do not directly apply to nonlinear theory. The breakdown of the principle of superposition is a possible means of detecting the presence of a nonlinear effect. Let $y_{1}(t)$ and $y_{2}(t)$ be the responses of a structure to the input forces $x_{1}(t)$ and $x_{2}(t)$, respectively. The principle of superposition is violated if $\alpha y_{1}(t)+\beta y_{2}(t)$ is not the structural response to the input $\alpha x_{1}(t)+\beta x_{2}(t)$. However, as explained in (Worden and Tomlinson, 2001 [67]), it is of limited practical utility, and simpler procedures should be employed.

The test for homogeneity which is a restricted form of the principle of superposition - $\beta$ is set to 0 - is one of the most popular detection technique. Homogeneity violation is best visualised in the frequency domain through distortions of FRFs. However, because a weaker condition is enforced, this linearity test is not infallible. An homogeneity test was applied to the ECL benchmark depicted in Fig. 1. Close-ups of FRFs measured at excitation levels of 1.4 and $22 \mathrm{Nrms}$ (see the solid and dotted lines, respectively) are shown in Fig. 14. Due to the geometric nonlinearity, severe distortions are introduced in the FRFs when the excitation level increases. Another means of inspecting FRFs for distortions indicative of nonlinearity is through visualisation of Nyquist plots which combine gain and phase characteristics in a single plot. For a linear system with well-separated modes, each resonance should generate the major part of a circle. In addition, the 

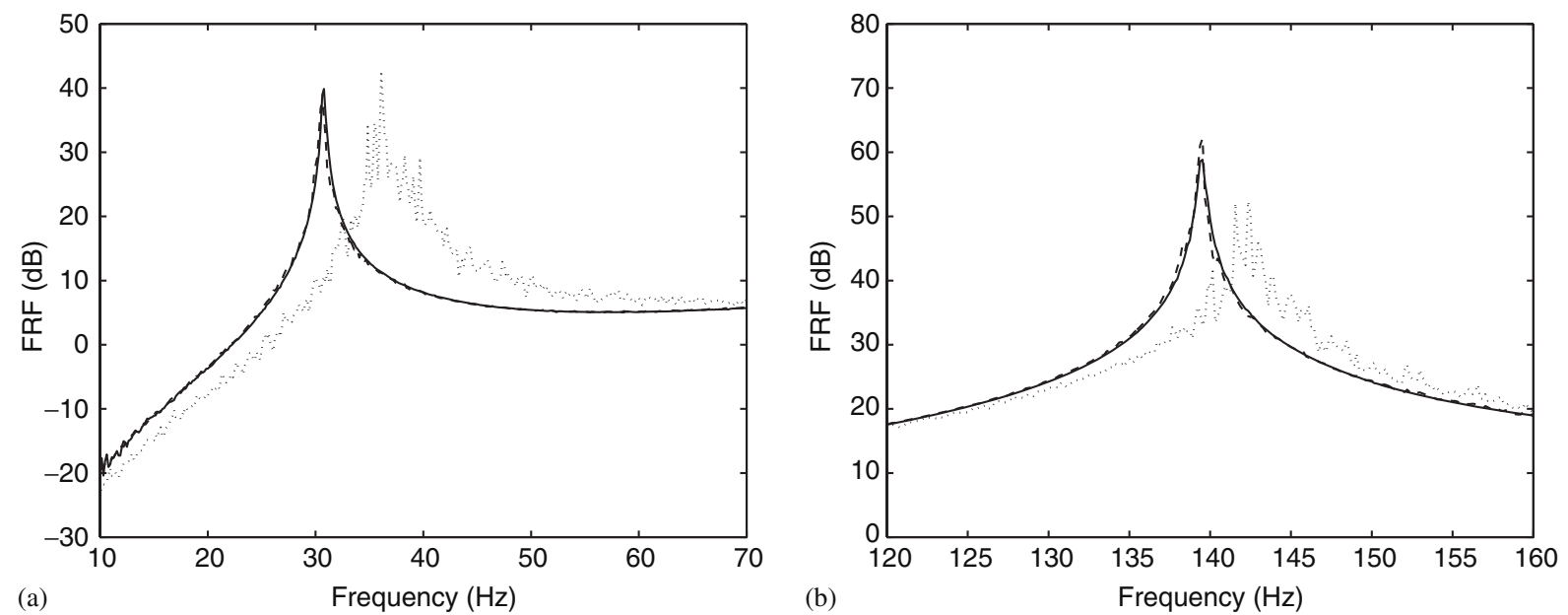

Fig. 14. Magnitude of $H_{73}$ (ECL benchmark): (a) first resonance; (b) second resonance. ( - , FRF measured using $H_{2}$ estimate at $1.4 \mathrm{Nrms}$ (the geometric nonlinearity is not activated); $\cdots \cdots$, FRF measured using $H_{2}$ estimate at $22 \mathrm{Nrms} ;---$, FRF measured using $H_{c 2}$ estimate at $22 \mathrm{Nrms})$.

isochrones (i.e., the lines connecting points of constant frequency for different excitation levels) should be straight lines for a linear system. If these two properties do not hold, a nonlinearity should be suspected.

Due to the symmetry of the dynamic compliance matrix $\mathbf{H}(\omega)$, to measure a FRF by impacting point $\mathrm{A}$ and measuring the response at point $\mathrm{B}$ is equivalent to measure a FRF by impacting point $\mathrm{B}$ and measuring the response at point $\mathrm{A}$. The reciprocity test is not infallible either because reciprocity can hold for symmetrical nonlinear systems. Also, as mentioned in (Wyckaert, 1992 [328]), interchanging exciter and transducer locations can introduce additional reciprocity violation errors due to misalignment of the shaker for instance.

The ordinary coherence function $\gamma$ (Newland, 1984 [332]) is normally used for assessing the quality of data measured under random excitation

$$
\gamma^{2}(\omega)=\frac{\left|S_{y x}(\omega)\right|^{2}}{S_{x x}(\omega) S_{y y}(\omega)}=\frac{H_{1}}{H_{2}} \quad \text { with } \quad H_{1}(\omega)=\frac{S_{y x}(\omega)}{S_{x x}(\omega)}, \quad H_{2}(\omega)=\frac{S_{y y}(\omega)}{S_{y x}(\omega)},
$$

where $S_{y y}(\omega), S_{x x}(\omega)$ and $S_{y x}(\omega)$ contain the power spectral density (PSD) of the response (e.g., acceleration signal), the PSD of the applied force and the cross PSD between the response and the applied force, respectively; $H_{1}$ and $H_{2}$ represent the so-called $H_{1}$ and $H_{2}$ FRF estimators. The coherence function is required to be unity for all accessible $\omega$ if and only if the system is linear and noise-free. Allemang and Brown (1987 [333]) suggested to utilise it as a detection tool for nonlinear behaviour because it is a rapid indicator of the presence of nonlinearity in specific frequency bands or resonance regions. It is arguably the most often-used test, by virtue of the fact that almost all the commercial spectrum analysers allow its calculation; however, it does not distinguish between the cases of a nonlinear system and noisy signals. The coherence function measured on the ECL benchmark at excitation levels of 1.4 and $22 \mathrm{Nrms}$ is depicted in Fig. 15. It is close to unity for the lowest excitation level for which the geometric nonlinearity is not activated whereas severe drops can be observed for the highest level confirming the presence of a nonlinearity.

A more sophisticated diagnostic tool introduced in (Simon and Tomlinson, 1984 [334]) is provided by the Hilbert transform $\mathscr{H}[\bullet]$ which diagnoses nonlinearity on the basis of measured FRF data. It merely exploits the fact that the FRF of a linear system is invariant under a Hilbert transformation. This is discussed in more details in Section 4.2. Another indicator based on the Hilbert transform is the corehence function $\lambda$ of Rauch (1992 [335])

$$
\lambda^{2}(\omega)=\frac{\left|\mathscr{H}[H(\omega)] H(\omega)^{*}\right|^{2}}{|\mathscr{H}[H(\omega)]|^{2}|H(\omega)|^{2}}
$$




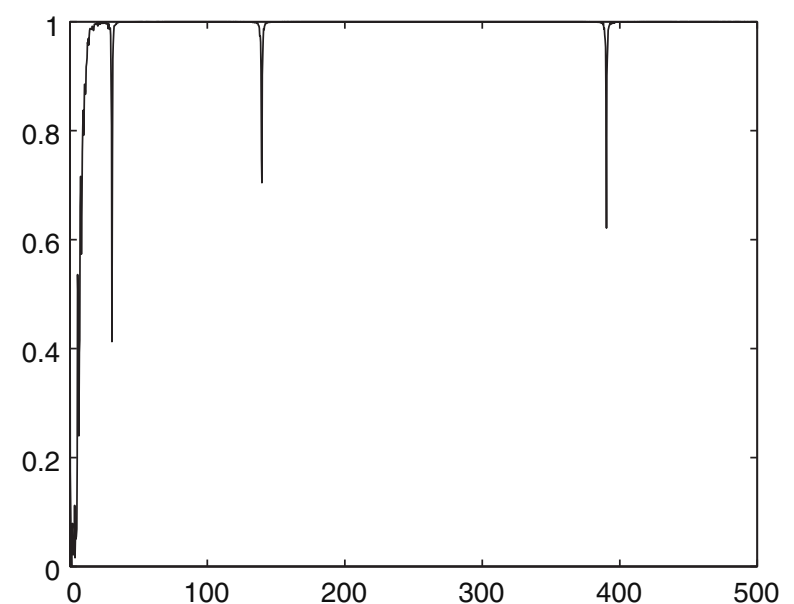

(a)

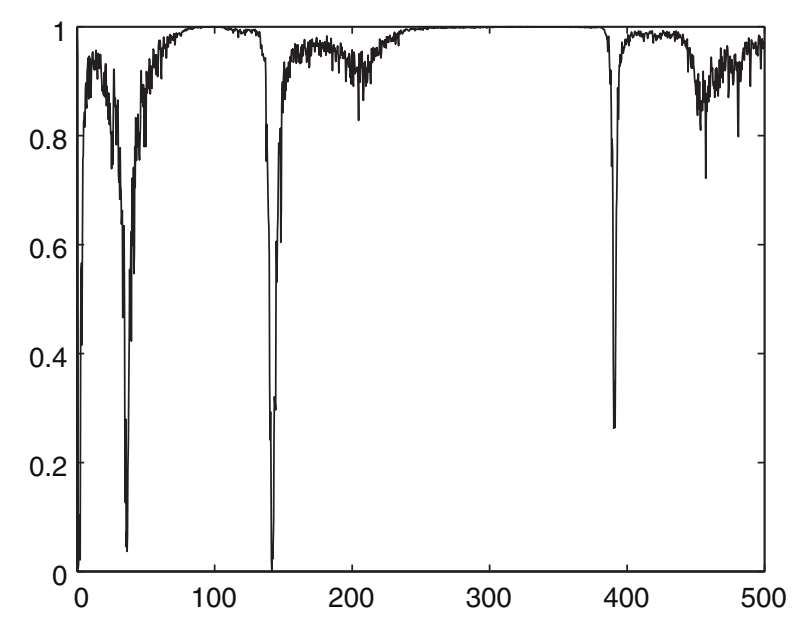

(b)

Fig. 15. Ordinary coherence function (ECL benchmark): (a) $1.4 \mathrm{Nrms}$; (b) $22 \mathrm{Nrms}$.

where star denotes the complex conjugate. It is defined to be unity for linear systems. The coherence function appears to be a more sensitive indicator of nonlinearity than the coherence. However, to some extent its utility is offset by its greater difficulty of calculation. As more commercial analysers with Hilbert transform facilities become available this objection should vanish.

Returning to the time domain, Billings and co-workers have established a number of simple correlation tests which can signal nonlinearity by manipulating measured time data. If records of both input $x$ and output $y$ are available, it can be shown that the correlation function

$$
\phi_{x^{2} y^{\prime}}(\tau)=E\left[x(t)^{2} y^{\prime}(t+\tau)\right]
$$

vanishes for all $\tau$ if and only if the system is linear (Billings and Tsang, 1990 [336]). The apostrophe signifies that the mean has been removed from the signal. If only sampled outputs are available, it can be shown that under certain conditions (Billings and Fadzil, 1985 [337]) the correlation function

$$
\phi_{y^{\prime} y^{\prime 2}}(k)=E\left[y^{\prime}(t+k)\left(y^{\prime}(t)\right)^{2}\right]
$$

is zero for all $k$ if and only if the system is linear. In practice these functions will never be identically zero; however, confidence intervals for a zero result can be calculated straightforwardly. As an example the correlation functions for acceleration data from a bilinear system at both low and high excitation are shown in Fig. 16; the dashed lines are the $95 \%$ confidence limits for a zero result. The function in Fig. $16 \mathrm{~b}$ indicates that the data from the high excitation test arises from a nonlinear system. The low excitation test did not excite the nonlinearity and the corresponding function (Fig. 16a) gives a null result as required.

Because harmonic distortion in nonlinear systems produces vibration response components at frequencies other than the excitation frequency, HOFRFs (Storer and Tomlinson, 1993 [192]) and higher-order spectra (Choi et al., 1984 [338]; Collis et al., 1998 [339]) have the capability to detect the presence of a nonlinear effect. Their advantage is that, besides nonlinearity detection, they can provide some qualitative information about the nonlinear behaviour (e.g., the type of the nonlinearity). One difficulty is that they are not easy to compute and measure. HOFRFs are discussed in detail in Section 6.5.

Another typical feature of nonlinear systems is that the frequency of motion is amplitude dependent. The plot which depicts the frequency as a function of the free vibration envelope is called a backbone curve; the presence of nonlinearity can be directly inferred from it. A backbone curve can be extracted using a timedomain version of the Hilbert transform (Feldman, 1994a [172]) in the case of a monocomponent signal with slowly varying amplitude (see also Section 6.4). For signals with multiple components, filtering efforts are needed, but this drawback has been recently removed with the introduction of the Huang-Hilbert transform 


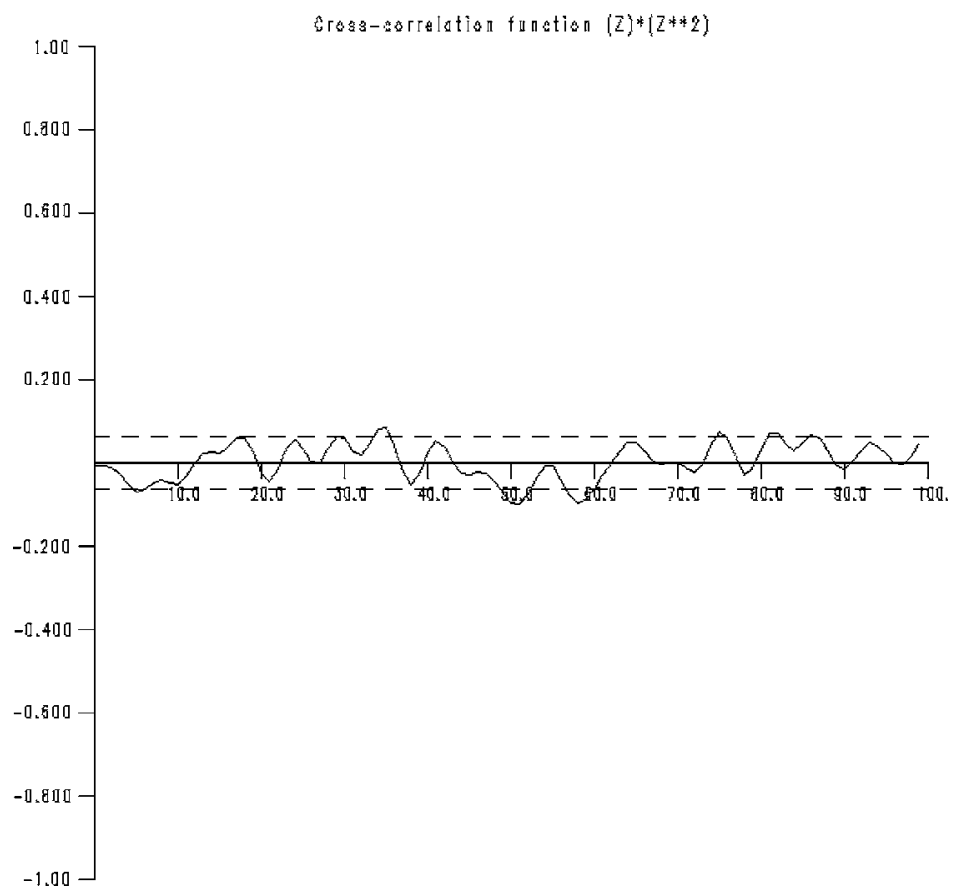

(a)

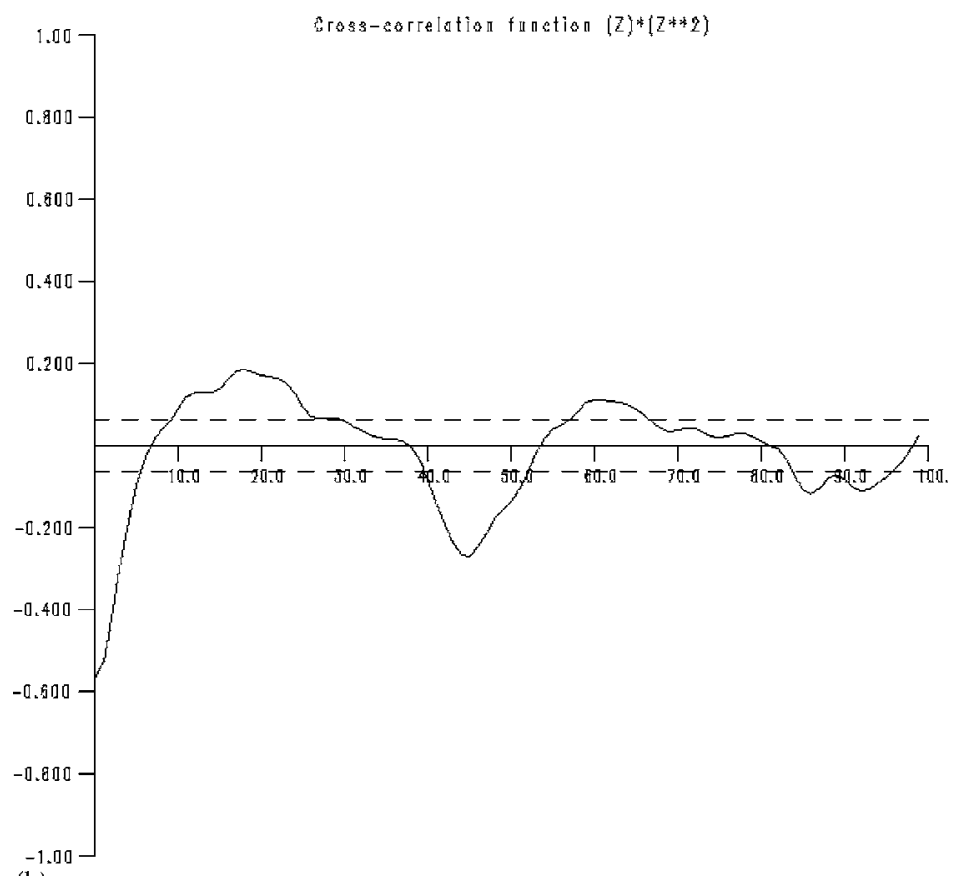

(b)

Fig. 16. Correlation tests: (a) linear system; (b) nonlinear system.

(Huang et al., 1998 [176]). Time-frequency analysis using the wavelet (Staszewski, 2000 [276]) or Gabor (Spina et al., 1996 [274]) transforms also offers a convenient means of computing a backbone curve.

Other detection techniques are the harmonic detection function (Van Der Auweraer et al., 1984 [340]; Mertens et al., 1986 [341]), the inverse receptance method (He and Ewins, 1987 [342]), the complex stiffness method (Mertens et al., 1989 [343]), the non-causal power ratio (Kim and Park, 1993 [344]) and carpet plots 
(Ewins, 2000 [11]). Recently developed techniques include the use of autocorrelation functions of residuals from overdetermined FRF calculations (Adams and Allemang, 2000b [345]) and the use of multisine excitations (Vanhoenacker et al., 2001 [346]; Verboven et al., 2005 [347]). In (Trendafilova et al., 2000 [348]), the nonlinearity detection procedure is recast into a classification problem using a nearest neighbour approach.

Finally, it is noted that damage causes a structure that initially behaves in a predominantly linear manner to exhibit nonlinear response when subject to its operating environment. The formation of cracks that subsequently open and close under operating loads is an example of such damage. The detection of nonlinearity may thus sometimes be related to the presence of a structural damage (Trendafilova and Van Brussel, 2001 [349]; Farrar et al., 2004 [350]).

\subsection{An example of nonlinearity detection: the Hilbert transform}

\subsubsection{Theory}

The Hilbert transform diagnoses nonlinearity on the basis of measured FRF data. The map on a FRF $H(\omega)$ is

$$
\mathscr{H}[H(\omega)]=\tilde{H}(\omega)=\frac{-1}{\mathrm{i} \pi} \int_{-\infty}^{\infty} \frac{H(\Omega)}{\Omega-\omega} \mathrm{d} \Omega .
$$

This mapping reduces to the identity on the FRFs of linear systems. For nonlinear systems, the Hilbert transform results in a distorted version $\tilde{H}(\omega)$ of the original FRF. This is illustrated in the Nyquist plot of Fig. 17 in the case of a hardening cubic stiffness; the characteristic circle is rotated clockwise and elongated into a more elliptical form. Distortions due to other types of nonlinearities (e.g, softening cubic stiffness and Coulomb friction) are discussed in (Worden and Tomlinson, 2001 [67]).

The origin of the distortion is described in (Worden and Tomlinson, 2001 [67]) and summarised here. Suppose $H(\omega)$ is decomposed so

$$
H(\omega)=H^{+}(\omega)+H^{-}(\omega),
$$

where $H^{+}(\omega)$ [respectively, $H^{-}(\omega)$ ] has poles only in the upper (respectively, lower) half of the complex $\omega$ plane. It can be shown that

$$
\mathscr{H}\left[H^{ \pm}(\omega)\right]= \pm H^{ \pm}(\omega) .
$$

The distortion suffered in passing from the FRF to the Hilbert transform is given by

$$
\Delta H(\omega)=\mathscr{H}[H(\omega)]-H(\omega)=-2 H^{-}(\omega) .
$$

A major problem in using the Hilbert transform on FRF data occurs when non-baseband (i.e, data which does not start at zero frequency) or band-limited data is employed. Practically speaking, all data falls into one of these categories, and the problem of neglecting the 'out of band' data always exists; the data over the intervals $\left(-\infty, \omega_{\min }\right)$ and $\left(\omega_{\max }, \infty\right)$ will be unavailable. By making use of the parity of the real and imaginary

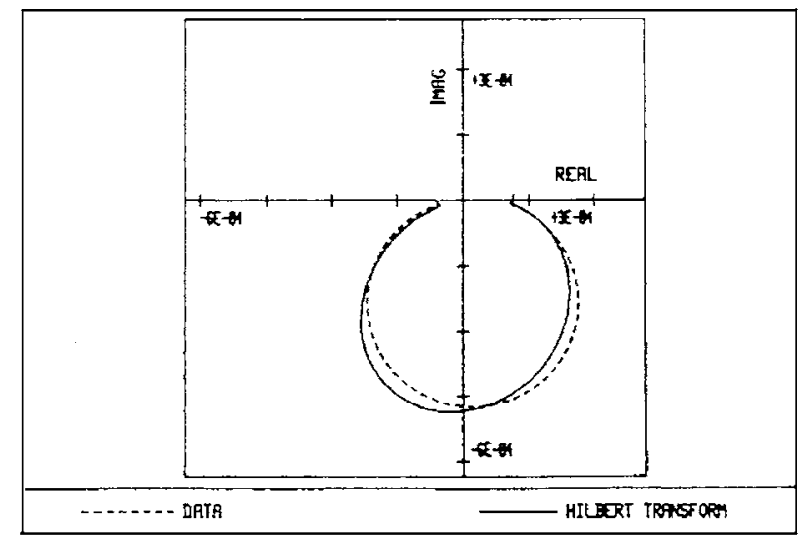

Fig. 17. Hilbert transform of a hardening cubic spring FRF at a low sine excitation level. 
parts of the FRF, the Hilbert transform can be recast in a slightly different form to that described above

$$
\begin{aligned}
& \mathfrak{R}[\tilde{H}(\omega)]=-\frac{2}{\pi} \int_{0}^{\infty} \frac{\mathfrak{J}[H(\Omega)] \Omega}{\Omega^{2}-\omega^{2}} \mathrm{~d} \Omega, \\
& \mathfrak{I}[\tilde{H}(\omega)]=\frac{2 \omega}{\pi} \int_{0}^{\infty} \frac{\mathfrak{R}[H(\Omega)]}{\Omega^{2}-\omega^{2}} \mathrm{~d} \Omega .
\end{aligned}
$$

If zoomed data from $\left(\omega_{\min }, \omega_{\max }\right)$ is measured, data is missing from the intervals $\left(0, \omega_{\min }\right)$ and $\left(\omega_{\max }, \infty\right)$.

The problem is usually overcome by adding correction terms to the Hilbert transform evaluated from $\omega_{\min }$ to $\omega_{\max }$ (Simon, 1983 [351]; Ahmed, 1987 [352]). An alternative approach establishes the position of the FRF poles in the complex planes and forms the decomposition (8). This is achieved by formulating a rational polynomial (RP) model of the FRF over the chosen frequency range and then converting this into the required form via a pole zero decomposition.

A general FRF may be expanded into a rational polynomial representation

$$
H(\omega)=\frac{Q(\omega)}{P(\omega)}=\frac{\sum_{i=0}^{n_{Q}} a_{i} \omega^{i}}{\sum_{i=0}^{n_{P}} b_{i} \omega^{i}} .
$$

The polynomial coefficients $a_{i}$ and $b_{i}$ are functions of the natural frequencies, dampings and participation factors of the modes. Once the RP model $H_{R P}$ is established, it can be converted into a pole-zero form

$$
H_{R P}(\omega)=\frac{\prod_{i=1}^{n_{Q}}\left(\omega-q_{i}\right)}{\prod_{i=1}^{n_{P}}\left(\omega-p_{i}\right)},
$$

where $q_{i}$ and $p_{i}$ are the (complex) zeroes and poles of the function, respectively. The next stage is a long division and partial fraction analysis in order to produce the decomposition (8)

$$
H_{R P}^{+}(\omega)=\sum_{i=1}^{N_{+}} \frac{C_{i}^{+}}{\omega-p_{i}^{+}}, \quad H_{R P}^{-}(\omega)=\sum_{i=1}^{N_{-}} \frac{C_{i}^{-}}{\omega-p_{i}^{-}},
$$

where $C_{i}^{+}$and $C_{i}^{-}$are coefficients fixed by the partial fraction analysis; $N_{+}$and $N_{-}$are the number of poles in the upper and lower half plane, respectively. Once this decomposition is established, the Hilbert transform follows from (10).

The procedure described above is demonstrated using data from a simulated Duffing oscillator system

$$
\ddot{y}+20 \dot{y}+10,000 y+5 \times 10^{9} y^{3}=X \sin (\omega t) \text {. }
$$

Data was generated over 256 spectral lines from 0 to $38.4 \mathrm{~Hz}$ in a simulated stepped-sine test. The data was truncated by removing data above and below the resonance leaving 151 spectral lines in the range 9.25-32.95 Hz. Fig. 18 shows the Hilbert transforms of the FRF calculated by the RP method on the truncated

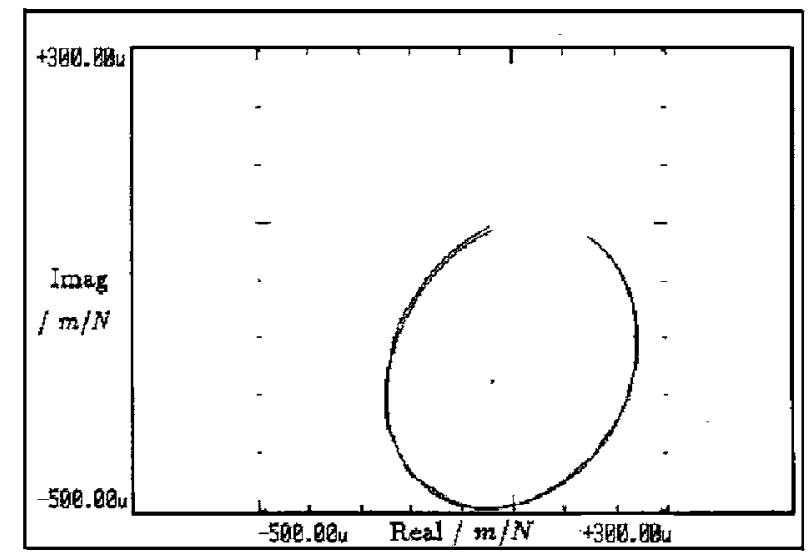

Fig. 18. Comparison of Hilbert Transforms from RP approach and standard integral. 
data and by a standard numerical method which used the full range of the data. The agreement between the two methods is good. Note also the characteristic clockwise rotation of the Nyquist plot for the hardening cubic stiffness system.

The pole-zero decomposition method can also be used to compute analytical expressions for the Hilbert transform as in (King and Worden, 1994 [353]).

\subsubsection{Assessment}

The Hilbert transform is a fast and effective means of testing for nonlinearity on the basis of a measured FRF. It has the advantage over a test of homogeneity, for example, in that it can be applied to a single FRF measured at a single level of excitation (as long as the nonlinearity has been adequately excited). Computation is fairly straightforward for a baseband FRF, but complications can arise for zoomed FRFs. However, the problems can be circumvented by the use of correction terms or a pole-zero computation. An appealing feature of the Hilbert transform is that the form of the distortion observed for a nonlinear system FRF can give some (limited) insight into the qualitative form of the nonlinearity. Perhaps the main limitation of the Hilbert transform is associated with all detection methods which look for distortion in a measured quantity; it is that there is currently no established technology to determine if the deviations observed in the FRF are statistically significant. As the Hilbert transform is usually used, the diagnosis of nonlinearity depends on expert judgment. A further (probably minor) concern is that the Hilbert transform does not strictly detect nonlinearity, but non-causality. It is not established beyond doubt that all nonlinear systems have noncausal FRFs (in the sense that their corresponding impulse responses have support for negative times) and this means that there may exist classes of nonlinear systems which the Hilbert transform would not detect.

\section{Characterisation of nonlinearity}

\subsection{Literature review}

According to the scheme in Fig. 4, the characterisation of nonlinearity is the second step toward the development of a structural model with a good predictive capability. Since the paper mainly focuses on parameter estimation techniques, the relevant methods for nonlinearity characterisation will solely be cited or very briefly described. In fact, this research topic could deserve its own survey paper.

A nonlinear system is said to be characterised when the location, type and functional form of all the nonlinearities throughout the system are determined. It is of crucial importance to have an accurate characterisation of the nonlinear elastic and dissipative behaviour of the physical structure prior to parameter estimation. Without a precise understanding of the nonlinear mechanisms involved, the identification process is bound to failure. Characterisation is a very challenging step because nonlinearity may be caused by many different mechanisms and may result in plethora of dynamic phenomena. This is evidenced in (Malatkar and Nayfeh, 2003c [354]) in which a simple cantilever plate may exhibit 2:1 and 3:1 internal resonances, external combination resonance, energy transfer between widely spaced modes, period-doubled motions and chaos.

\subsubsection{The location of the nonlinearity}

The spatial localisation of local nonlinearities is the first step in the characterisation process. The literature on this topic is not so extensive because nonlinearities may often be located easily, at least for simple structures. Some information may be gleaned by studying FRFs at various excitation levels and examining the deformation shapes of the modes which are most corrupted by the nonlinear response; nonlinearities may be assumed where the relative displacements of these mode shapes are the largest. Other procedures have been developed, e.g., procedures based on the RFS method (Al-Hadid and Wright, 1989 [138]), test-analysis correlation (Lin and Ewins, 1995 [355]), error localisation in a linear model updating framework (see, e.g., (Fritzen et al., 1998 [356]; Pascual et al., 1999 [357])), pattern recognition (Trendafilova et al., 2000 [348]) and scanning laser vibrometry (Vanlanduit et al., 2000 [358]). 


\subsubsection{The type of the nonlinearity}

Nonlinearity classification is useful for determining the type of the nonlinearity. It amounts to answering several questions: (i) does the nonlinearity come from stiffness or damping (or both)? (ii) does the system have hardening or softening characteristics? (iii) is the restoring force symmetric or asymmetric? (iv) is the nonlinearity weak or strong? and (v) is the restoring force smooth or non-smooth?

Answers to some of these questions may be provided by looking at the distortions in measured FRFs of nonlinear systems using Bode plots (see Fig. 14), Nyquist plots (Vakakis and Ewins, 1994 [359]), Volterra series and HOFRFs (Storer and Tomlinson, 1993 [192]; Schoukens et al., 2000 [360]; Chatterjee and Vyas, 2001 [361]), frequency-domain ARX (Auto-Regressive with eXogenous inputs) models (Adams, 2002 [362]) and modulation matrices (Adams and Allemang, 1999b [234]) [FRFs of nonlinear systems are discussed at length in (Nayfeh and Mook, 1979 [28]; Worden and Tomlinson, 2001 [67])]. Because one class of nonlinearity can behave like another in a certain input-output amplitude range, the shape of the FRF is not always conclusive evidence of a particular nonlinearity.

The form of the distortion introduced during a Hilbert transform of the FRF can also be characteristic of the type of nonlinearity (Simon and Tomlinson, 1984 [334]; Worden and Tomlinson, 2001 [67]). In the case of a hardening cubic stiffness, the peak of the Hilbert transform curve in the Bode plot appears at a higher frequency than in the FRF; the peak magnitude of the Hilbert transform is also higher. The time-domain version of the Hilbert transform described in Section 6.4 also provides some insight into the nonlinearity. The hardening or softening characteristic of the system can be easily deduced from the backbone curve which depicts the frequency as a function of the free vibration envelope. Information about damping can be inferred from the damping curve and the signal envelope; e.g, it is well known that Coulomb friction has an envelope with linear decay. The same kind of information can be gathered using the wavelet transform (Staszewski, 2000 [276]; Argoul and Le, 2003 [281]) and the Gabor transform (Franco and Pauletti, 1997 [275]). For instance, characterisation of nonlinearities of an aeroelastic system using the wavelet transform is performed in (Lind et al., 2001 [363]).

Higher-order spectra yield information about a signal's non-Gaussianity; e.g, a Gaussian input to a nonlinear system produces a non-Gaussian output (Collis et al., 1998 [339]). For symmetrically distributed inputs and restoring forces with only odd terms, the output has a symmetric distribution and consequently a zero bispectrum. The trispectrum provides further information in this case and enables a quantitative measure of the strength of the nonlinearity. In (Hajj et al., 2000 [204]) the phase obtained from the bispectrum is used for nonlinearity characterisation.

The RFS method (Masri and Caughey, 1979 [101]) has 'built-in characterisation capabilities' as discussed in Section 5.2. By representing the restoring force as a function of the displacement and velocity in a threedimensional plot, the nonlinearity can be conveniently visualised. A characterisation of the elastic and dissipative forces can be obtained by taking a cross section of this three-dimensional plot along the axes where either the velocity or the displacement is equal to zero, respectively.

\subsubsection{The functional form of the nonlinearity}

A priori knowledge and nonlinearity classification may help to select a reasonably accurate model of the nonlinearity. If little is known about the form of the model before starting the identification process, one may resort to polynomial expansion. The principle of polynomial expansion is to approximate the restoring force by a polynomial of some degree in displacement and velocity (coupling terms may also be included). The number of possible terms increases rapidly with the polynomial order, but most often not all terms in the expansion have a significant contribution to the restoring force. Indicators such as the significance factor (Atkins and Worden, 1997 [364]) and coherence functions (Richards and Singh, 1999 [227]; Bendat and Piersol, 2000 [109]) provide a means of determining which terms are significant and which terms can be safely discarded. However, there may be several disadvantages to using ordinary polynomial series in practice:

- The restoring forces of real structures are not necessarily governed by integer power series. For instance, non-integer exponent-type models were successful at describing the nonlinear behaviour of a rubber isolator (Richards and Singh, 2001 [19]), of a vehicle suspension (Adams and Allemang, 2000c [365]) and of the ECL benchmark (Kerschen et al., 2003a [229]; Lenaerts et al., 2003 [264]; see also Sections 7.1.2 and 
7.4.2). Fractional derivatives have also enjoyed some success for modeling viscoelastic materials (Schmidt and Gaul, 2003 [366]).

- When using high-order polynomial expansions, several models may be equally good, and overfitting may also be an issue. An algorithm based on a Bayesian inference approach may alleviate these drawbacks (Kerschen et al., 2003b [367]). The key advantage of the method is that a collection of potential models together with their posterior probability is obtained instead of the single best model; this allows for more flexibility in deciding the most appropriate model of the nonlinearity.

- Poor numerical conditioning may occur for polynomial series which contain both small and large powers. Normalised and pseudo-orthogonal generating functions do not have this limitation (Adams and Allemang, 2000c [365]).

- There are classes of nonlinearity that cannot be modeled accurately even with high-order polynomial expansions (e.g, non-smooth nonlinearities).

Analytical studies and experiments are also of great utility to model the nonlinear structural behaviour. Structural systems exhibiting inelastic restoring forces with hereditary characteristics are widely encountered in the field of mechanics. Due to the hysteretic nature of the restoring force, the nonlinear force cannot be expressed in the terms of the state variables of the system. As a result, much effort has been devoted to develop models of hysteretic restoring forces (Iwan, 1966 [368]; Iwan, 1967 [369]; Bouc, 1967 [370]; Wen, 1976 [371]; Baber and Noori, 1985 [372]). Recent results on modeling and analysis of hysteretic systems are found in (Vestroni and Noori, 2002 [373]). Joints and interfaces (Goodman, 1959 [374]; Groper, 1985 [375]; Gaul and Lenz, 1997 [376]; Hartwigsen et al., 2004 [377]; Song et al., 2004 [301]), breathing cracks (Ibrahim et al., 1987 [378]; Worden et al., 1994b [296]; Sundermeyer and Weaver, 1995 [379]; Friswell and Penny, 2002 [380]; Luzzato, 2003 [381]), and materials such as rubber and polyurethane foams (White et al., 2000 [16]; Schultze et al., 2001 [17]; Richards and Singh, 2001 [19]; Singh et al., 2003 [18]) can produce interesting and complicated nonlinear dynamics. Their successful modeling largely depends on understanding and reproducing their basic physics. We also mention that the rising need for additional damping in fast developing fields such as aerospace industries has led to the development of new technologies with highly nonlinear mechanical properties (e.g., particle dampers and hard ceramic coatings); their modeling may be a challenging task (Liu et al., 2002 [382]; Wong et al., 2004 [383]).

Even if a priori information and physics-based models should not be superseded by any 'blind' methodology, the lack of knowledge about nonlinearity may be circumvented by nonlinear black-box models such as those proposed in (Kosmatopoulos et al., 2001 [298]; Fan and Li, 2002 [303]; Peifer et al., 2003 [305]). These models are prepared to describe virtually any nonlinear dynamics on the basis of the data alone, but the identified parameters have little or no physical meaning. Problems may also arise when choosing a too simplistic or too complex model; however, this issue is also encountered in parametric methods.

\subsection{An example of nonlinearity characterisation: the restoring force surface method}

The RFS procedure which is described in Section 6.1 has been applied to the characterisation and identification of automotive shock absorbers in a number of publications (see Section 3.2).

The results presented in Fig. 19 are for a number of sets of test data from a FIAT vehicle shock absorber. The data was obtained by FIAT engineers using the experimental facilities of the vehicle test group at Centro Ricerche FIAT, Torino. The apparatus and experimental strategy are described in (Belingardi and Campanile, 1990 [150]); the subsequent data processing and analysis can be found in (Surace et al., 1992 [151]). Briefly, data was recorded from an absorber which was constrained to move in only one direction in order to justify the assumption of SDOF behaviour. The top of the absorber was fixed to a load cell so that the internal force could be measured directly (it was found that inertial forces were negligible). The base was then excited harmonically using a hydraulic actuator. The absorber was tested at six frequencies, 1, 5, 10, 15, 20, and $30 \mathrm{~Hz}$; the results shown here are for the $10 \mathrm{~Hz}$ test.

The RFS and the associated contour map are given in Fig. 19, they both show a very clear bilinear characteristic. On the contour map, the contours, which are concentrated in the positive velocity half-plane, are almost parallel and inclined at a small angle to the $\dot{y}=0$ axis showing that the position dependence of the 


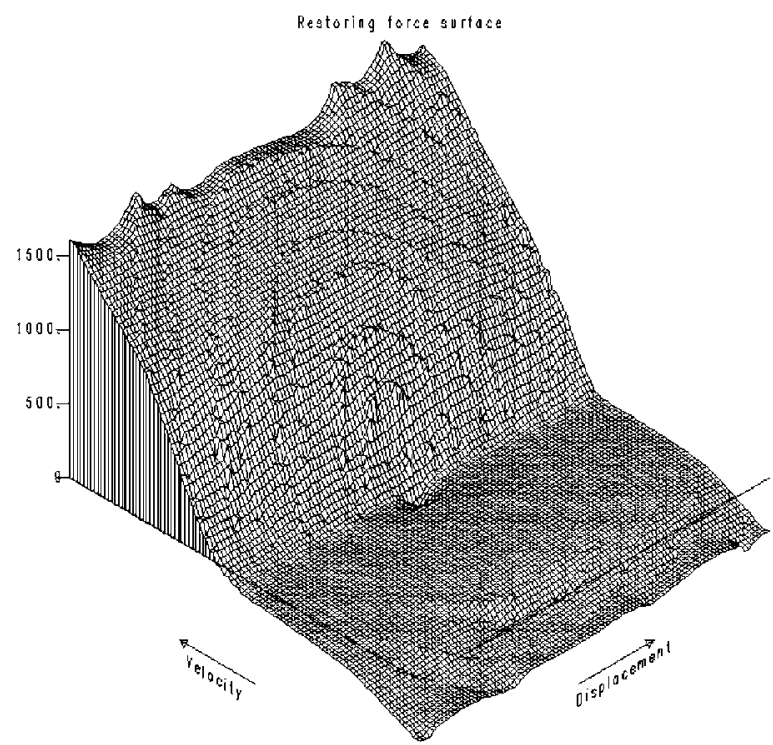

(a)

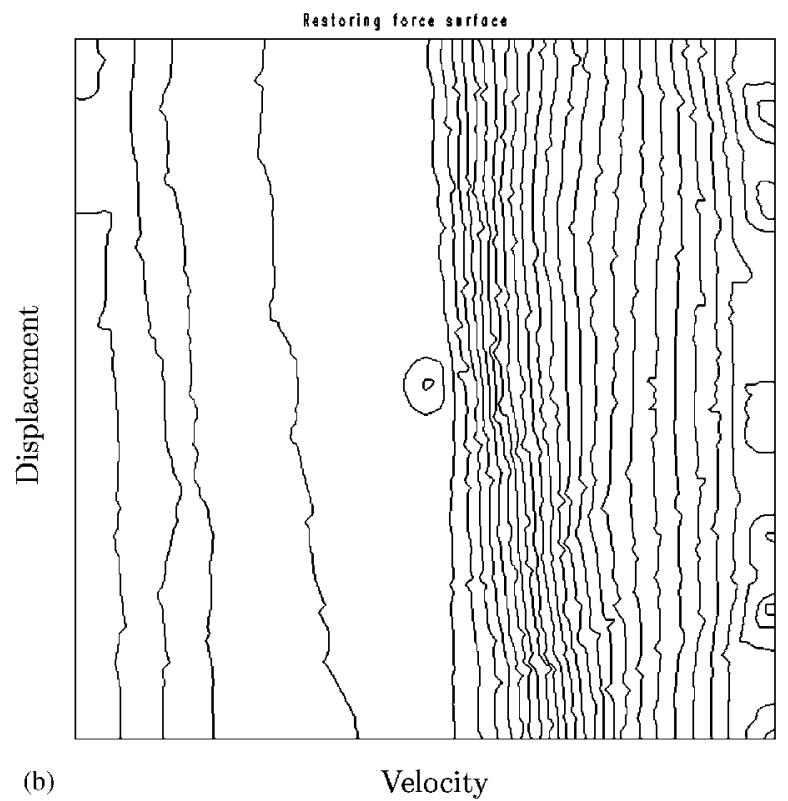

Fig. 19. Experimental restoring force surface for an automotive shock absorber: (a) surface; (b) contour plot.

absorber is small and essentially linear. Note that if a parametric representation of the internal force had been obtained, say a least-squares polynomial, it would have been impossible to infer the bilinear characteristic from the coefficients alone; it is the direct visualisation of the nonlinearity which makes the force surface so useful.

The surfaces from the tests at other frequencies showed qualitatively the same characteristics, i.e., a small linear stiffness and a bilinear damping. However, the line of discontinuity in the surface was found to rotate in the phase plane as the test frequency increased. A simple analysis using differenced force surfaces showed that this dependence on frequency was not simply a consequence of disregarding the absorber mass (Worden and Tomlinson, 1992 [384]). Force surfaces have also been used to investigate the temperature dependence of shock absorbers (Surace et al., 1992 [151]).

\section{Parameter estimation in the presence of nonlinearity: established methods}

Parameter estimation is the last step toward the establishment of a structural model with a good predictive accuracy as shown in Fig. 4. An important assumption which conditions the success of parameter estimation is that all the nonlinearities throughout the system have been properly characterised.

In this section, several established methods for parameter estimation in the presence of nonlinearity are described. Numerical and/or experimental examples are presented to illustrate their fundamental concepts but also their assets and limitations.

\subsection{The restoring force surface method}

\subsubsection{Theory}

The simple procedure described in this section allows a direct identification for SDOF nonlinear systems. The basic procedures were introduced by Masri and Caughey (1979 [101]), although the approach described here resembles more the variant developed independently by Crawley and Aubert (1986 [133]) and Crawley and O'Donnell (1986 [132]) and referred to as force-state mapping. A recent variation on the theme is the local approach of Duym and Schoukens (1996b [154]) which fits a piecewise linear RFS. 
The starting point is the equation of motion as specified by Newton's second law

$$
m \ddot{y}+f(y, \dot{y})=x(t),
$$

where $m$ is the mass (or an effective mass) of the system, and $f(y, \dot{y})$ is the internal restoring force which acts to return the absorber to equilibrium when disturbed. The function $f$ can be a quite general function of position $y(t)$ and velocity $\dot{y}(t)$. Because $f$ is assumed to be dependent only on $y$ and $\dot{y}$ it can be represented by a surface over the phase-plane, i.e., the $(y, \dot{y})$ plane. A trivial re-arrangement of Eq. (17) gives

$$
f(y, \dot{y})=x(t)-m \ddot{y} .
$$

Now, if the mass $m$ is known, and the excitation $x(t)$ and acceleration $\ddot{y}(t)$ are measured, all the quantities on the right-hand side of this equation are known, and hence so is $f$. As usual, measurement of a time signal entails sampling it at regularly spaced intervals $\Delta t$. If $t_{i}=(i-1) \Delta t$ denotes the $i$ th sampling instant, then at $t_{i}$, Eq. (18) gives

$$
f_{i}=f\left(y_{i}, \dot{y}_{i}\right)=x_{i}-m \ddot{y}_{i},
$$

where $x_{i}=x\left(t_{i}\right)$ and $\ddot{y}_{i}=\ddot{y}\left(t_{i}\right)$, and hence $f_{i}$ is known at each sampling instant. If the velocities $\dot{y}_{i}$ and displacements $y_{i}$ are also known (i.e., from direct measurement or from numerical integration of the sampled acceleration data), at each instant $i=1, \ldots, N$ a triplet $\left(y_{i}, \dot{y}_{i}, f_{i}\right)$ is specified. The first two values indicate a point in the phase plane, the third gives the height of the RFS above that point. Given this scattering of force values above the phase plane there are a number of methods of interpolating a continuous surface on a regular grid (one is Sibson's natural neighbor method, implemented in the commercial software package TILE4 (Sibson, 1985 [385])). There are a couple of other issues of signal processing here. In the first case, direct sampling of the displacement, velocity and acceleration data requires considerable instrumentation. It is more economical to measure one and estimate the remaining states by numerical differentiation or integration; the issues which arise in this strategy are addressed in (Worden, 1990a [141]). This problem was neatly avoided in (Shin and Hammond, 1998a [144]), where the authors adopted a state-space embedding approach and fitted a force surface of the form $f_{i}=f\left(y_{i}, y_{i-1}\right)$. A second issue is the choice of excitation signal to give uniform coverage of the phase plane; this is addressed in (Worden, 1990b [142]; Duym and Schoukens, 1995 [66]).

Once the surface is obtained, Masri and Caughey (1979 [101]) construct a model of the restoring force in the form of a double Chebyshev series

$$
f(y, \dot{y})=\sum_{i=0}^{m} \sum_{j=0}^{n} C_{i j} T_{i}(y) T_{j}(\dot{y}),
$$

where $T_{i}(y)$ is the Chebyshev polynomial of order $i$. It has since been established (Al-Hadid and Wright, 1989 [138]; Worden and Tomlinson, 1989 [386]) that a straightforward polynomial expansion of the form

$$
f(y, \dot{y})=\sum_{i=0}^{m} \sum_{j=0}^{n} C_{i j} y^{i} \dot{y}^{j}
$$

is superior in terms of ease, speed and accuracy of estimation. The only advantage of the Chebyshev form of the expansion is that the coefficients can be estimated independently of each other due to the fact that the polynomials are orthogonal (Masri and Caughey, 1979 [101]).

There is a class of systems for which the RFS method cannot be used in the simple form described above; i.e., systems with memory or hysteretic systems. In this case, the internal force does not depend entirely on the instantaneous position of the system in the phase plane. As an illustration, consider the Bouc-Wen model (Bouc, 1967 [370]; Wen, 1976 [371])

$$
\begin{aligned}
& m \ddot{y}+f(y, \dot{y})+z=x(t), \\
& \dot{z}=-\alpha|\dot{y}| z \cdot|z|^{n-1}-\beta \dot{y}\left|z^{n}\right|+A \dot{y},
\end{aligned}
$$

which can represent a broad range of hysteresis characteristics. The RFS method would fail here because the internal force is a function of $y, \dot{y}$ and $z$; this means that the force surface over $(y, \dot{y})$ would appear to be multivalued. A smooth surface can be obtained by exciting the system at a single frequency over a range of 
amplitudes; however, the surfaces would be different for each frequency. Extensions of the method to cover hysteretic systems have been devised by Lo and Hammond (1988 [146]) and Benedettini et al. (1991 [147]); models of the type

$$
\dot{f}=g(f, \dot{y})
$$

are obtained which also admit a representation as a surface over the $(f, \dot{y})$ plane. A parametric approach to modeling hysteretic systems was pursued in (Yar and Hammond, 1987 [387]) where a Bouc-Wen model (22)-(23) was fitted to measured data; this approach is complicated by the fact that model (22)-(23) is nonlinear in the parameters.

\subsubsection{Application example}

There exist in the literature a number of examples of the application of force surface techniques to experimental systems. One of the first is that of Crawley and O'Donnell (1986 [132]) which includes a study of space-structure joints. Worden and Tomlinson (1991 [388]) consider an impacting cantilever beam as does the more recent study by Kerschen et al. (2001 [156]). Meskell et al. (2001 [157]) applied the approach to a nonlinear fluid-loading example. The experimental study of Hunter et al. (1989 [389]) is also of interest in that it contains a frequency domain formulation of the method. The RFS procedure has also been applied to the characterisation and identification of automotive shock absorbers in a number of publications (see, e.g., (Audenino et al., 1990 [149]; Belingardi and Campanile, 1990 [150]; Surace et al., 1992 [151])).

The example chosen to illustrate the method is the impacting cantilever beam in Fig. 3 (Kerschen et al., 2001 [156]). The beam was excited using a band-limited white noise centered on its first natural frequency (see Fig. 20a), $18 \mathrm{~Hz}$, and behaved as a SDOF system. The acceleration shown in Fig. 20b was measured at the beam tip, and the displacement and velocity were deduced from this signal using integration and filtering procedures. A piecewise linear model

$$
f(y, \dot{y})= \begin{cases}k y+c \dot{y}+k_{+}(y-d)+c_{+}\left(\dot{y}-\dot{y}_{y=d}\right) & \text { if } y \geqslant d, \\ k y+c \dot{y} & \text { if }|y|<d, \\ k y+c \dot{y}+k_{-}(y+d)+c_{-}\left(\dot{y}-\dot{y}_{y=-d}\right) & \text { if } y \leqslant-d\end{cases}
$$

was fitted to the data, which allowed the estimation of the parameters $k, c, k_{+}, c_{+}, k_{-}, c_{-}$. By looking for the minimum value of the normalised mean-square error (NMSE (Allen, 1971 [390])) between the predicted and measured restoring force for variations of the clearance $d$, this parameter could have been identified; the smallest NMSE was $1.80 \%$, which is the sign of an accurate identification. Fig. 21 presents the comparison between the measured stiffness curve (i.e., a cross section of the three-dimensional surface corresponding to
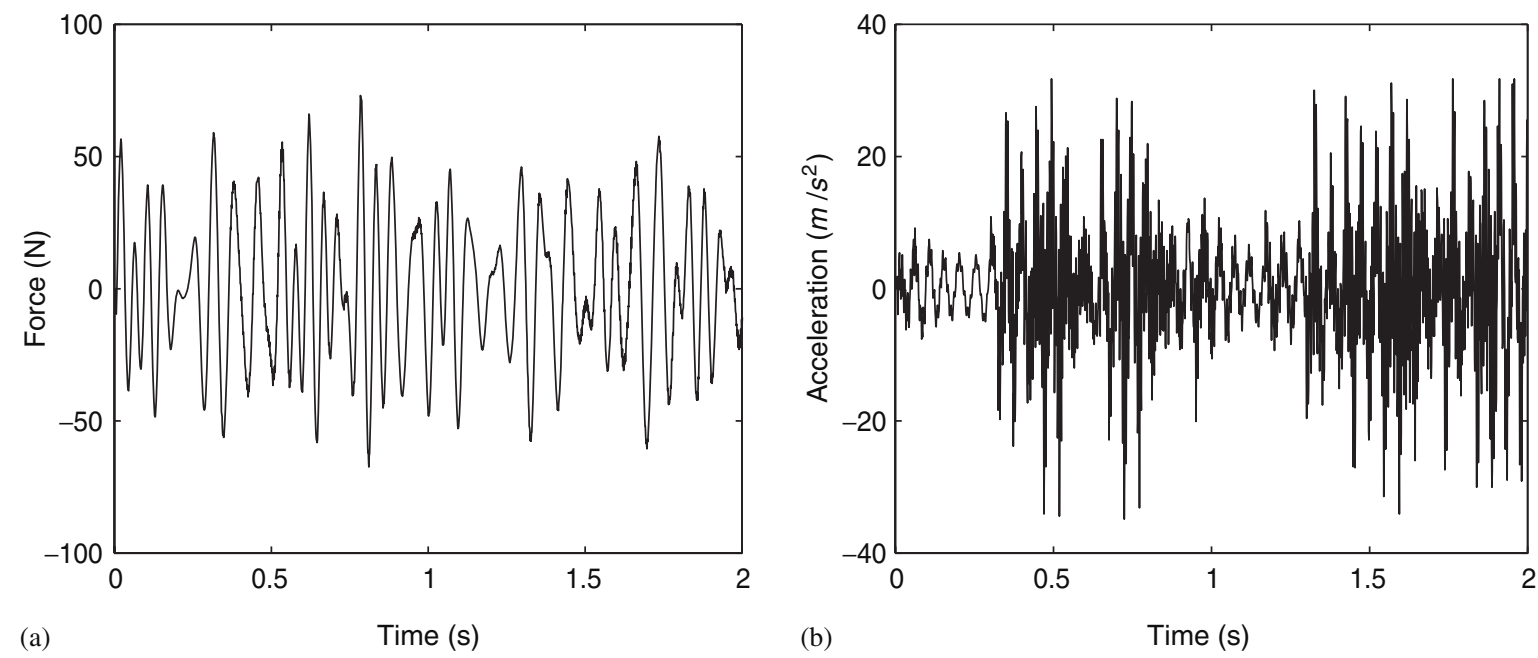

Fig. 20. Measured signals: (a) force time history; acceleration time history. 


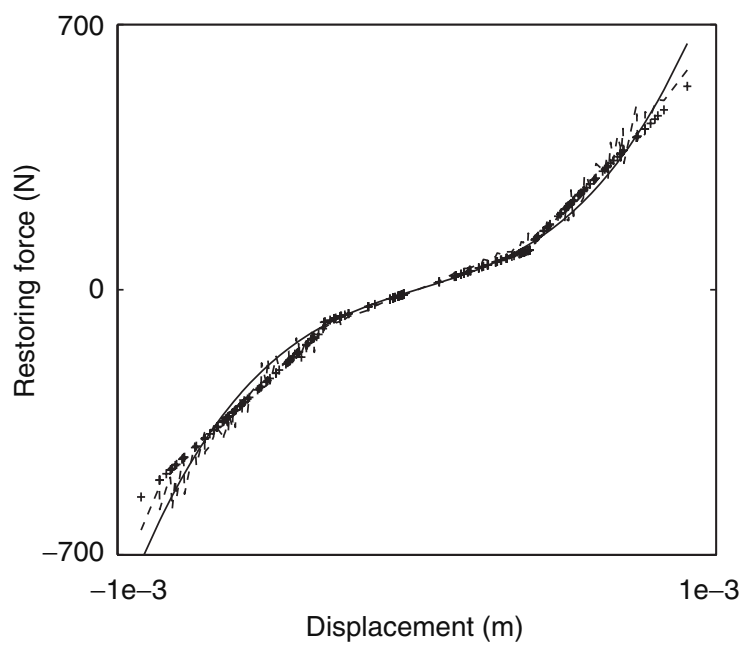

Fig. 21. Measured and reconstructed stiffness curves: - - - , measured; +, reconstructed (piecewise linear model); —-, reconstructed (polynomial model).

zero velocities) and the reconstructed curve given by the piecewise linear model. A polynomial model was also fitted to the experimental data in (Kerschen et al., 2001 [156]); its stiffness curve is also displayed in Fig. 21.

\subsection{Direct parameter estimation and restoring force surfaces}

The identification procedure of Masri and Caughey (1979 [101]) which was discussed in the previous section was shown to extend to MDOF systems in (Masri et al., 1982 [134]). Although in principle arbitrarily complex nonlinear systems could be identified, in practice, the computational burden was considerable. Attempts to obtain a practical implementation of the procedure were made in (Worden and Tomlinson, 1988 [391]; A1Hadid and Wright, 1989 [138]). The main difficulty was that the identification procedure was carried out in modal coordinates, the intention being to simplify matters by diagonalising the underlying linear system. The procedure therefore required a priori estimates of the modal matrix and mass matrix. Although the linear parts of the restoring forces were simplified by this procedure, each component of the nonlinear restoring force vector remained a function of all the coordinates. Al-Hadid and Wright showed that unless a time-consuming iterative version of the procedure was adopted, any model parameters would be biased. A further problem is that RFSs can no longer be obtained before the parameter estimation stage.

However, research continued, and in Al-Hadid and Wright (1989, [138]) a useful form of the identification procedure was obtained by utilising a physical coordinate representation for the nonlinear forces while retaining a modal coordinate approach to the underlying linear system. This can be contrasted with the later work of Masri et al. (1987a,b [136,137]) where physical coordinates are used for the linear identification and modal coordinates for the nonlinear. As described in the previous section, it was also found that simple polynomial expansions are preferable to the double Chebyshev expansions of Masri and Caughey. In subsequent papers by Al-Hadid and Wright (1990, 1992 [139,140]), experimental results are presented for a MDOF system, and a powerful technique for obtaining estimates of the mass and modal mass matrices appears. Al-Hadid (1989 [392]), Worden (1989 [393]) and Wright and Al-Hadid (1991 [394]) present a number of results relating to the sensitivity of the identification procedure to measurement errors.

In the remainder of this section, an alternative approach to MDOF system identification is described. Rather than make any use of modal coordinates, a physical coordinate system based on a lumped parameter representation of the system is adopted. Although this is now a direct parameter estimation (DPE) scheme similar to that adopted by Masri et al. $(1987 \mathrm{a}, \mathrm{b}[136,137])$ for linear systems, it will be shown that all system 
parameters can be obtained if the system is excited at a single point. Also, restoring forces are shown to be a useful by-product. An alternative approach to DPE is described in (Mohammad et al., 1991 [143]).

\subsubsection{Theory}

For a general $N$ DOF system, it is assumed that the mass is concentrated at $N$ measurement points, $m_{i}$ being the mass at point $i$. Each point $i$ is then assumed to be connected to each other point $j$ by a link $l_{i j}$, and to ground by a link $l_{i i}$. The situation is illustrated in Fig. 22 for a three-DOF system.

If the masses are displaced and released, they are restored to equilibrium by internal forces in the links. These forces are assumed to depend only on the relative displacements and velocities of the masses at each end of the links. If $\delta_{i j}=y_{i}-y_{j}$ is the relative displacement of mass $m_{i}$ relative to mass $m_{j}$, and $\dot{\delta}_{i j}=\dot{y}_{i}-\dot{y}_{j}$ is the corresponding relative velocity, then

Force in link $l_{i j}:=f_{i j}\left(\delta_{i j}, \dot{\delta}_{i j}\right)$,

where $\delta_{i i}=y_{i}$ and $\dot{\delta}_{i i}=\dot{y}_{i}$ for the link to ground. It will be clear that, as links $l_{i j}$ and $l_{j i}$ are the same

$$
f_{i j}\left(\delta_{i j}, \dot{\delta}_{i j}\right)=-f_{j i}\left(\delta_{j i}, \dot{\delta}_{j i}\right)=-f_{j i}\left(-\delta_{i j},-\dot{\delta}_{i j}\right)
$$

If an external force $x_{i}(t)$ is now applied at each mass, the equations of motion are

$$
m_{i} \ddot{y}_{i}+\sum_{j=1}^{N} f_{i j}\left(\delta_{i j}, \dot{\delta}_{i j}\right)=x_{i}(t) \quad i=1, \ldots, N .
$$

It is expected that this type of model would be useful for representing a system with a finite number of modes excited. In practice, only the $N$ accelerations and input forces at each point are measured. Differencing

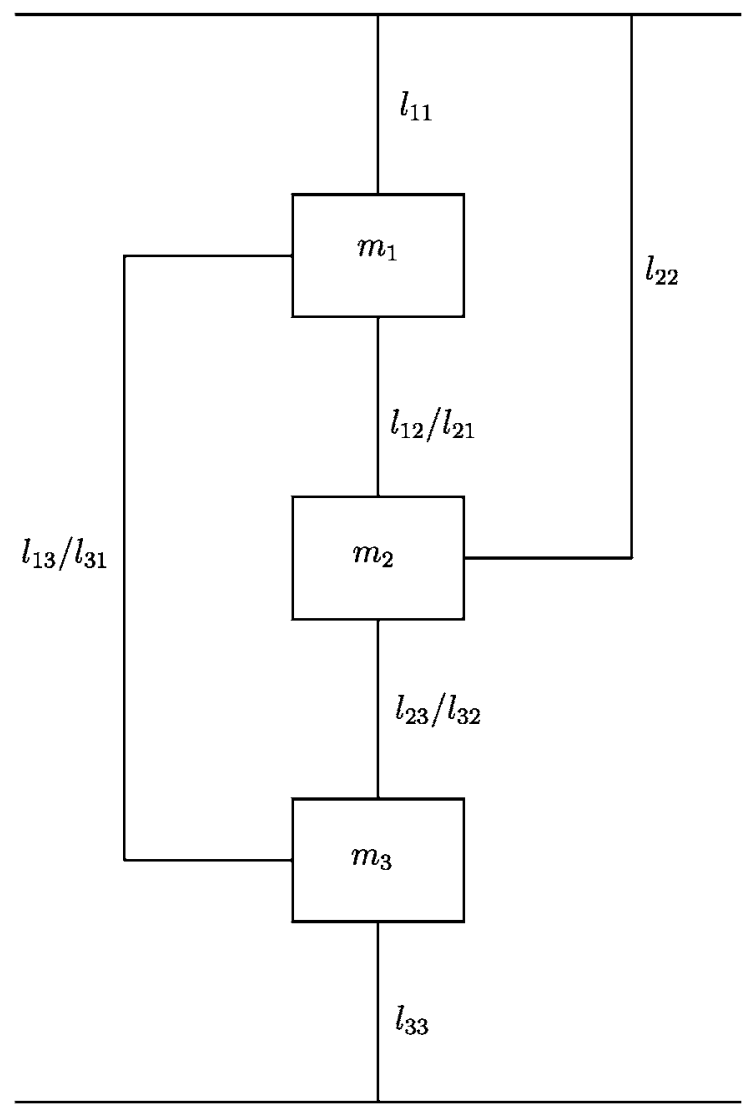

Fig. 22. Link model for a 3DOF system. 
yields the relative accelerations $\ddot{\delta}_{i j}$ which can be integrated numerically to give $\dot{\delta}_{i j}$ and $\delta_{i j}$. A polynomial representation is adopted here for $f_{i j}$ giving a model

$$
m_{i} \ddot{y}_{i}+\sum_{j=1}^{N} \sum_{k=0}^{p} \sum_{l=0}^{q} a_{(i j) k l}\left(\delta_{i j}\right)^{k}\left(\dot{\delta}_{i j}\right)^{l}=x_{i} .
$$

Least-squares parameter estimation can be used to obtain the values of the coefficients $m_{i}$ and $a_{(i j) k l}$ which best fit the measured data. Note that an a priori estimate of the mass is not required. If there is no excitation at point $i$, transmissibility arguments yield the appropriate form for the equation of motion of $m_{i}$

$$
f_{i j}^{\prime}\left(\delta_{i j}, \dot{\delta}_{i j}\right)=\sum_{j=1}^{N} \sum_{k=0}^{p} \sum_{l=0}^{q} a_{(i j) k l}^{\prime}\left(\delta_{i j}\right)^{k}\left(\dot{\delta}_{i j}\right)^{l}=-\ddot{y}_{i},
$$

where $a_{(i j) k l}^{\prime}=a_{(i j) k l} / m_{i}$.

In terms of the expansion coefficients, the symmetry relation (27) becomes

$$
a_{(i j) k l}=(-1)^{k+l+1} a_{(j i) k l}
$$

or

$$
m_{i} a_{(i j) k l}^{\prime}=(-1)^{k+l+1} m_{j} a_{(j i) k l}^{\prime} .
$$

In principle, the inclusion of difference variables allows the model to locate nonlinearity (Al-Hadid and Wright, 1989 [138]); for example, if a term of the form $\left(\delta_{23}\right)^{3}$ appears in the appropriate expansion one can infer the presence of a cubic stiffness nonlinearity between points 2 and 3 .

Suppose now that only one of the inputs $x_{i}$ is non-zero. Without loss of generality it can be taken as $x_{1}$. The equations of motion become

$$
\begin{aligned}
& m_{1} \ddot{y}_{1}+\sum_{j=1}^{N} f_{i j}\left(\delta_{i j}, \dot{\delta}_{i j}\right)=x_{1}(t), \\
& \ddot{y}_{i}+\sum_{j=1}^{N} f_{i j}^{\prime}\left(\delta_{i j}, \dot{\delta}_{i j}\right)=0 \quad i=2, \ldots, N .
\end{aligned}
$$

One can identify all coefficients in the $\ddot{y}_{2}$ equation up to an overall scale-the unknown $m_{2}$ which is embedded in each $f_{2 j}^{\prime}$. Similarly, all the coefficients in the $\ddot{y}_{3}$ equation can be known up to the scale $m_{3}$. Multiplying the latter coefficients by the ratio $m_{2} / m_{3}$ would therefore scale them with respect to $m_{2}$. This means that coefficients for both equations are known up to the same scale $m_{2}$. The ratio $m_{2} / m_{3}$ can be obtained straightforwardly; if there is a link $l_{23}$ the two equations will contain terms $f_{23}^{\prime}$ and $f_{32}^{\prime}$. Choosing one particular term, e.g., the linear stiffness term, from each $f^{\prime}$ expansion gives, via (32)

$$
\frac{m_{2}}{m_{3}}=\frac{a_{(32) 10}^{\prime}}{a_{(23) 01}^{\prime}} .
$$

The scale $m_{2}$ can then be transferred to the $\ddot{y}_{4}$ equation coefficients by the same method if there is a link $l_{24}$ or $l_{34}$. In fact, the scale factor can be propagated through all the equations since each mass point must be connected to all other mass points through some sequence of links. If this were not true the system would fall into two or more disjoint pieces.

If the $\ddot{y}_{1}$ equation has an input, $m_{1}$ is estimated and this scale can be transferred to all equations so that the whole MDOF system can be identified using only one input. Yang and Ibrahim (1985 [135]) observed that if the unforced equations of motion are considered, the required overall scale can be fixed by a knowledge of the total system mass; i.e., all system parameters can be obtained from measurements of the free oscillations.

If a restriction is made to linear systems, the symmetry relations in (27) yield the reciprocity relation. By assuming that reciprocity holds at the outset, it is possible to identify all system parameters using one input by an alternative method which is described in Mohammad et al. (1991 [143]). 
A further advantage of adopting this model is that it allows a natural definition of the RFS for each link. After obtaining the model coefficients the surface $f_{i j}$ can be plotted as a function of $\delta_{i j}$ and $\dot{\delta}_{i j}$ for each link $l_{i j}$. In this case the surfaces are purely a visual aid to the identification, and are more appropriate in the nonlinear case.

This approach is illustrated on an experimental system in (Worden et al., 1994c [395]). The DPE scheme has also been implemented for distributed systems in (Liang and Cooper, 1992 [396]).

\subsubsection{Assessment of the RFS and DPE methods}

Although the RFS and DPE methods can deal with MDOF nonlinear systems, they are essentially appealing because of their simplicity and efficiency for identification of SDOF systems or nonlinearity localised between two DOFs. These methods offer a convenient means of determining the functional form of the nonlinearity through the visualisation of the RFS as shown in Section 5.2. A characterisation of the elastic and dissipative forces can be obtained by taking a cross section of this three-dimensional plot along the axes where either the velocity or the displacement is equal to zero, respectively. A difficulty lies in the need for numerical differentiation or integration which may introduce errors in the estimation of signals; careful signal processing is required.

\subsection{NARMAX modeling}

\subsubsection{Theory}

Suppose one is interested in the SDOF linear system

$$
m \ddot{y}+c \dot{y}+k y=x(t) .
$$

This can be converted by a process of discrete approximation to the discrete-time form

$$
y_{i}=a_{1} y_{i-1}+a_{2} y_{i-2}+b_{1} x_{i-1},
$$

where $a_{1}, a_{2}$ and $b_{1}$ are constant coefficients and functions of the original parameters $m, c, k$ and the sampling interval $\Delta t=t_{i+1}-t_{i}$ where the $t_{i}$ are the sampling instants. In a more general form

$$
y_{i}=F\left(y_{i-1}, y_{i-2} ; x_{i-1}\right) \text {. }
$$

This is an $A R X$ (Auto-Regressive with eXogenous inputs) model. The advantage of adopting this form is that only the two states $x$ and $y$ need to be measured in order to estimate all the model parameters $a_{1}, a_{2}$ and $b_{1}$ in (38) and thus identify the system. It is a simple matter to show that a general MDOF linear system has a discrete-time representation

$$
y_{i}=\sum_{j=1}^{n_{y}} a_{j} y_{i-j}+\sum_{j=1}^{n_{x}} b_{j} x_{i-j}
$$

or

$$
y_{i}=F\left(y_{i-1}, \ldots, y_{i-n_{y}} ; x_{i-1}, \ldots, x_{i-n_{x}}\right) \text {. }
$$

As before, all parameters $a_{1}, \ldots, a_{n_{y}}, b_{1}, \ldots, b_{n_{x}}$ can be estimated using measurements of the $x$ and $y$ data only.

The extension to nonlinear systems is straightforward. Consider the Duffing oscillator represented by

$$
m \ddot{y}+c \dot{y}+k y+k_{3} y^{3}=x(t) .
$$

One can pass to the discrete-time representation

$$
y_{i}=a_{1} y_{i-1}+a_{2} y_{i-2}+b_{1} x_{i-1}+c y_{i-1}^{3} \text {. }
$$

Model (42) is now termed a NARX (Nonlinear ARX) model. The regression function $y_{i}=F\left(y_{i-1}, y_{i-2} ; x_{i-1}\right)$ is now nonlinear; it contains a cubic term. If all terms of order three or less were included in the model structure, i.e., $\left(y_{i-2}\right)^{2} x_{i-1}$, etc., a much more general model would be obtained

$$
y_{i}=F^{(3)}\left(y_{i-1}, y_{i-2} ; x_{i-1}\right)
$$


(the superscript denotes the highest-order product terms) which would be sufficiently general to represent the behaviour of any dynamical systems with nonlinearities up to third order, i.e., containing terms of the form $\dot{y}^{3}$, $\dot{y}^{2} y$, etc.

The most general polynomial NARX model (including products of order $\leqslant n_{p}$ ) is denoted by

$$
y_{i}=F^{\left(n_{p}\right)}\left(y_{i-1}, \ldots, y_{i-n_{y}} ; x_{i-1}, \ldots, x_{i-n_{x}}\right) .
$$

It has been proved by Leontaritis and Billings $(1985 \mathrm{a}, \mathrm{b}[162,163])$ under very mild assumptions that any input-output process has a representation by a model of form (44). If the system nonlinearities are polynomial in nature, this model will represent the system well for all levels of excitation. If the system nonlinearities are not polynomial, they can be approximated arbitrarily accurately by polynomials over a given range of their arguments (Weierstrass approximation theorem in (Simmons, 1963 [397])). This means that the system can be accurately modeled by taking the order $n_{p}$ high enough. However, the model would be input-sensitive as the polynomial approximation required would depend on the data. This problem can be removed by including non-polynomial terms in the NARX model as described in (Billings and Chen, 1989d [398]). The NARX model can even be cast as a neural network (Billings et al., 1991a,b [166,399]).

The preceding analysis unrealistically assumes that the measured data is free of noise. As shown below, if the system is nonlinear the noise process can be very complex; multiplicative noise terms with the input and output are not uncommon, but can be easily accommodated in the discrete-time models as described in (Leontaritis and Billings, 1985a,b [162,163]; Korenberg et al., 1988 [164]; Chen et al., 1989 [400]).

Suppose the measured output has the form

$$
y(t)=y_{c}(t)+\zeta(t)
$$

where $y_{c}(t)$ is the 'clean' output from the system. If the underlying system is the Duffing oscillator of Eq. (41), the equation satisfied by the measured data is now

$$
m \ddot{y}+c \dot{y}+k y+k_{3} y^{3}-m \ddot{\zeta}-c \dot{\zeta}-k \zeta-k_{3} \zeta^{3}-3 y^{2} \zeta+3 y \zeta^{2}=x(t)
$$

and the corresponding discrete-time equation will contain terms of the form $\zeta_{i-1}, \zeta_{i-2}, \zeta_{i-1} y_{i-1}^{2}$, etc. Note that even simple additive noise on the output introduces cross-product terms if the system is nonlinear. Although these terms all correspond to unmeasurable states they must be included in the model. If they are ignored the parameter estimates will generally be biased. The system model (44) is therefore extended again by the addition of a noise model and takes the form

$$
y_{i}=F^{(3)}\left(y_{i-1}, y_{i-2} ; x_{i-1} ; \zeta_{i-1}, \zeta_{i-2}\right)+\zeta_{i} .
$$

This type of model is referred to as NARMAX (Nonlinear Auto-Regressive Moving-Average with eXogenous inputs). The NARMAX model was introduced in (Leontaritis and Billings, 1985a,b [162,163]).

Finally, the term moving-average requires some explanation. Generally, for a linear system a movingaverage model for the noise process takes the form

$$
\zeta_{i}=e_{i}+c_{1} e_{i-1}+c_{2} e_{i-2}+\cdots,
$$

i.e., the system noise is assumed to be the result of passing a zero-mean white noise sequence $\left\{e_{i}\right\}$ through a digital filter with coefficients $c_{1}, c_{2}$, etc. The terminology comes from the literature of time series analysis. Eq. (40) requires a generalisation of this concept to the nonlinear case. This is incorporated in the NARMAX model which takes the final general form

$$
y_{i}=F^{\left(n_{p}\right)}\left(y_{i-1}, \ldots, y_{i-n_{y}} ; x_{i-1}, \ldots, x_{i-n_{x}} ; e_{i-1}, \ldots, e_{i-n_{e}}\right)+e_{i} .
$$

In this form the noise sequence or residual sequence $e_{i}$ is now zero-mean white noise. This allows the model to accommodate a wide class of possibly nonlinear noise terms.

The input and output variables $x_{i}$ and $y_{i}$ are usually physical quantities like force and displacement response, respectively. An interesting alternative approach to this was followed by Thouverez and Jezequel (1996 [171]), who fitted a NARMAX model using modal coordinates.

Having obtained a NARMAX model for a system, the next stage in the identification procedure, i.e., model validity, is to determine if the structure is correct and the parameter estimates are unbiased. It is important to 
know if the model has successfully captured the system dynamics so that it will provide good predictions of the system output for different input excitations, or if it has simply fitted the model to the data; in which case it will be of little use since it will only be applicable to one data set. Three basic tests of the validity of a model have been established by Billings et al., they are described below in increasing order of stringency. In the following, $y_{i}$ denotes a measured output while $\hat{y}_{i}$ denotes an output value predicted by the model.

One-step-ahead predictions: Given the NARMAX representation of a system

$$
y_{i}=F^{\left(n_{p}\right)}\left(y_{i-1}, \ldots, y_{i-n_{y}} ; x_{i-1}, \ldots, x_{i-n_{x}} ; e_{i-1}, \ldots, e_{i-n_{e}}\right)+e_{i}
$$

the one-step-ahead prediction of $y_{i}$ is made using measured values for all past inputs and outputs. Estimates of the residuals are obtained from the expression $\hat{e}_{i}=y_{i}-\hat{y}_{i}$, i.e.,

$$
\hat{y}_{i}=F^{\left(n_{p}\right)}\left(y_{i-1}, \ldots, y_{i-n_{y}} ; x_{i-1}, \ldots, x_{i-n_{x}} ; \hat{e}_{i-1}, \ldots, \hat{e}_{i-n_{e}}\right) .
$$

The one-step-ahead series can then be compared to the measured outputs. Good agreement is clearly a necessary condition for model validity. An objective measure of the goodness of fit can be obtained using the NMSE.

Model predicted output: In this case, the inputs are the only measured quantities used to generate the model output, i.e.,

$$
\hat{y}_{i}=F^{\left(n_{p}\right)}\left(\hat{y}_{i-1}, \ldots, \hat{y}_{i-n_{y}} ; x_{i-1}, \ldots, x_{i-n_{x}} ; 0, \ldots, 0\right) .
$$

The zeroes are present because the prediction errors will not generally be available when one is using the model to predict output. In order to avoid a misleading transient at the start of the record for $\hat{y}$, the first $n_{y}$ values of the measured output are used to start the recursion. As above, the estimated outputs must be compared with the measured outputs, with good agreement a necessary condition for accepting the model. It is clear that this test is stronger than the previous one; in fact the one-step-ahead predictions can be excellent in some cases when the model predicted output shows complete disagreement with the measured data.

Correlation tests: These represent the most stringent of the validity checks. The appropriate reference is (Billings et al., 1989c [165]). The correlation function $\phi_{u v}(k)$ for two sequences of data $u_{i}$ and $v_{i}$ is defined by

$$
\phi_{u v}=E\left(u_{i} v_{i+k}\right) \approx \frac{1}{N-k} \sum_{i=1}^{N-k} u_{i} v_{i+k} .
$$

For a linear system the necessary conditions for model validity are

$$
\phi_{e e}(k)=\delta_{0 k}
$$

and

$$
\phi_{x e}(k)=0 \quad \forall k
$$

The first of these conditions is true only if the residual sequence $e_{i}$ is a white noise sequence. It is essentially a test of the adequacy of the noise model whose job is to reduce the residuals to white noise. If the noise model is correct, the system parameters should be free from bias. The second of the conditions above states that the residual signal is uncorrelated with the input sequence $x_{i}$, i.e., the model has completely captured the component of the measured output which is correlated with the input. Another way of stating this requirement is that the residuals should be unpredictable from the input.

In the case of a nonlinear system it is sometimes possible to satisfy the requirements above even if the model is invalid. It is shown in (Billings et al., 1989c [165]) that an exhaustive test of the fitness of a nonlinear model requires the evaluation of three additional correlation functions. The extra conditions are

$$
\begin{aligned}
& \phi_{e(e x)}(k)=0 \quad \forall k \geqslant 0, \\
& \phi_{x^{2^{\prime}} e}(k)=0 \quad \forall k, \\
& \phi_{x^{2^{\prime}} e^{2}}(k)=0 \quad \forall k .
\end{aligned}
$$


The dash which accompanies $x^{2}$ above indicates that the mean has been removed. Normalised estimates of all the correlation functions above are usually obtained so that confidence limits for a null result can be added.

\subsubsection{Assessment}

The NARMAX modeling technique is very versatile. In most cases, it can exploit well-established linearalgebraic means of least-squares estimation (the exception being for neural network NARX models, for example, which are nonlinear-in-the-parameters). The possibility of using a noise model makes it very powerful, although stability of the iterative procedure that fits the noise model can sometimes be a practical issue. If the method has a disadvantage, it is that the models do not directly give insight into the physics of the system being modeled, although it is possible to pass to a continuous-time model from the NARMAX model by using the HOFRFs. On the latter point, the NARMAX approach gives the fastest and least data-intensive means of computing the HOFRFs by harmonic probing of the parametric models. Another appealing feature of the NARMAX approach is the availability of nonlinear model validation criteria based on correlation tests.

\subsection{The Hilbert transform}

\subsubsection{Theory}

The frequency-domain Hilbert transform has been used for nonlinearity detection (see Section 4.2), but the time-domain version of the method has also enjoyed some success as a direct method of non-parametric identification. The method described in this section is the result of a programme of research by Feldman (1985, 1994a,b [401,172,173]). It provides a means of obtaining the stiffness and damping characteristics of SDOF systems. There are essentially two approaches, one based on free vibration FREEVIB and one on forced vibration FORCEVIB. Only FREEVIB is discussed here. Note that Feldman uses the traditional definition of the analytic signal and time-domain Hilbert transform throughout his analysis (this differs from the frequency-domain object defined elsewhere in this review by a factor of $-i$ ).

Consider a SDOF nonlinear system under free vibration

$$
\ddot{y}+h(\dot{y}) \dot{y}+\omega_{0}^{2}(y) y=0 .
$$

The object of the exercise is to deduce the forms of the nonlinear damping function $h(\dot{y})$ and nonlinear stiffness $k(y)=\omega_{0}^{2}(y)$.

The method is based on the analytic signal

$$
Y(t)=y(t)+\mathrm{i} \tilde{y}(t),
$$

where $\tilde{y}$ is the Hilbert transform of $y(t)$. The approach uses the magnitude and phase representation

$$
Y(t)=A(t) \mathrm{e}^{\mathrm{i} \psi(t)},
$$

where $A(t)$ is the instantaneous magnitude or envelope, and $\psi(t)$ is the instantaneous phase, both are real functions so

$$
y(t)=A(t) \cos (\psi(t)), \quad \tilde{y}=A(t) \sin (\psi(t))
$$

and,

$$
\begin{aligned}
& A(t)=\sqrt{y(t)^{2}+\tilde{y}(t)^{2}}, \\
& \psi(t)=\tan ^{-1}\left(\frac{\tilde{y}(t)}{y(t)}\right) .
\end{aligned}
$$

So both envelope and phase are available as functions of time if $y(t)$ is known and $\tilde{y}(t)$ can be computed. The derivatives can also be computed, either directly or using the relations

$$
\dot{A}(t)=\frac{y(t) \dot{y}(t)+\tilde{y}(t) \dot{\tilde{y}}(t)}{\sqrt{y(t)^{2}+\tilde{y}(t)^{2}}}=A(t) \Re\left[\frac{\dot{Y}(t)}{Y(t)}\right],
$$




$$
\omega(t)=\dot{\psi}(t)=\frac{y(t) \dot{\tilde{y}}(t)-\dot{y}(t) \tilde{y}(t)}{y(t)^{2}+\tilde{y}(t)^{2}}=\mathfrak{J}\left[\frac{\dot{Y}(t)}{Y(t)}\right],
$$

where $\omega(t)$ is the instantaneous frequency, again a real signal. The last two equations can be used to generate the first two derivatives of the analytic signal

$$
\begin{aligned}
& \dot{Y}(t)=Y(t)\left[\frac{\dot{A}(t)}{A(t)}+\mathrm{i} \omega(t)\right], \\
& \ddot{Y}(t)=Y(t)\left[\frac{\ddot{A}(t)}{A(t)}-\omega(t)^{2}+2 i \frac{\dot{A}(t) \omega(t)}{A(t)}+\mathrm{i} \dot{\omega}(t)\right] .
\end{aligned}
$$

Now, consider the equation of motion (59), with $h(\dot{y}(t))=h(t)$ and $\omega_{0}^{2}(y(t))=\omega_{0}^{2}(t)$ considered purely as functions of time. Because the functions $h$ and $\omega_{0}^{2}$ will generally be low-order polynomials of the envelope $A$, they will have a lowpass characteristic. If the resonant frequency of the system is high, $y(t)$ will, roughly speaking, have a highpass characteristic. This means that $h$ and $y$ can be considered as non-overlapping signals as can $\omega_{0}^{2}$ and $y$. If the Hilbert transform is taken of (59), it will pass through the functions $h$ and $\omega_{0}^{2}$. Further, the transform commutes with differentiation, so

$$
\ddot{\tilde{y}}+h(t) \dot{\tilde{y}}+\omega_{0}^{2}(t) \tilde{y}=0 .
$$

Adding (59) and $i \times(69)$ yields a differential equation for the analytic signal $Y$, i.e.,

$$
\ddot{Y}+h(t) \dot{Y}+\omega_{0}^{2}(t) Y=0
$$

or, the quasi-linear form

$$
\ddot{Y}+h(A) \dot{Y}+\omega_{0}^{2}(A) Y=0 .
$$

Now, the derivatives $\ddot{Y}$ and $\dot{Y}$ are known functions of $A$ and $\omega$ by (67) and (68). Substituting yields

$$
Y\left[\frac{\ddot{A}}{A}-\omega^{2}+\omega_{0}^{2}+h \frac{\dot{A}}{A}+\mathrm{i}\left(2 \omega \frac{\dot{A}}{A}+\dot{\omega}+h \omega\right)\right]=0 .
$$

Separating out the real and imaginary parts gives

$$
\begin{aligned}
& h(t)=-2 \frac{\dot{A}}{A}-\frac{\dot{\omega}}{\omega}, \\
& \omega_{0}^{2}(t)=\omega^{2}-\frac{\ddot{A}}{A}-h \frac{\dot{A}}{A},
\end{aligned}
$$

or

$$
\omega_{0}^{2}(t)=\omega^{2}-\frac{\ddot{A}}{A}+2 \frac{\dot{A}^{2}}{A^{2}}+\frac{\dot{A} \dot{\omega}}{A \omega}
$$

and these are the basic equations of the theory.

Suppose the free vibration is induced by an impulse, the subsequent response of the system will take the form of a decay. $y(t)$ can be measured and $\tilde{y}$ can then be computed. This means that $A(t)$ and $\omega(t)$ are available by using (63) and (64) and numerically differentiating $\psi(t)$.

Now, consider how the damping function is obtained. $h(t)$ is known from (73). As $A(t)$ is monotonically decreasing (energy is being dissipated), the inverse function $t(A)$ is single-valued and can be obtained from the graph of $A(t)$ against time (Fig. 23). The value of $h(A)$ is simply the value of $h(t)$ at $t(A)$ (Fig. 24). Similarly, the stiffness function is obtained via the sequence $A \longrightarrow t(A) \longrightarrow \omega_{0}^{2}(t(A))=\omega_{0}^{2}(A)$. The inverse of the latter mapping $A(\omega)$ is sometimes referred to as the backbone curve of the system. 


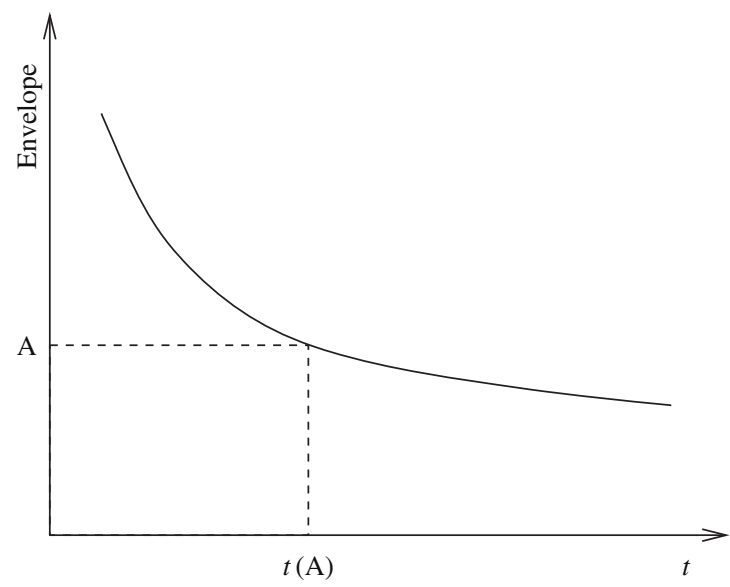

Fig. 23. Envelope used in Feldman's method.

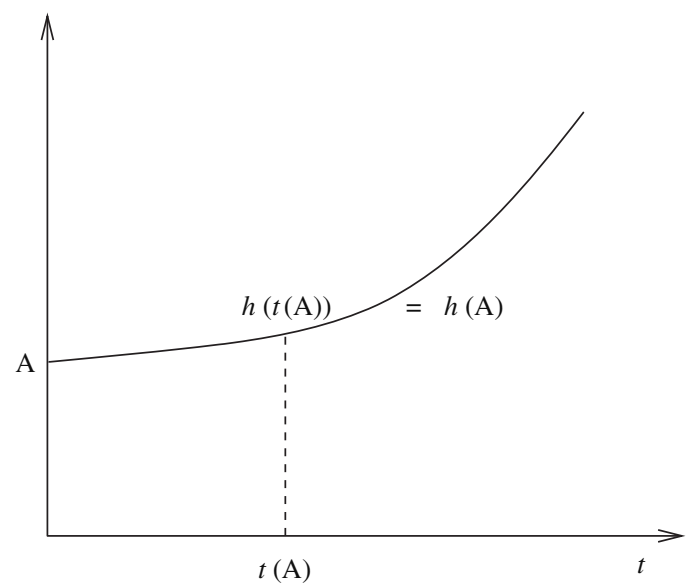

Fig. 24. Damping curve for Feldman's method.

Once $h(A)$ and $\omega_{0}^{2}(A)$ are known, the damper and spring characteristics $f_{d}(A)$ and $f_{s}(A)$ can be obtained trivially

$$
\begin{aligned}
& f_{d}(A)=\omega(A) A h(A), \\
& f_{s}(A)=A \omega_{0}^{2}(A) .
\end{aligned}
$$

Note that there are no assumptions on the forms of $f_{d}$ and $f_{s}$, the method is truly non-parametric. However, once the graphs $A \longrightarrow f_{d}$, etc., have been obtained, linear least-squares methods suffice to estimate parameters.

The method is illustrated here using data from numerical simulation. ${ }^{4}$ The first system is a Duffing oscillator with equation of motion

$$
\ddot{y}+10 \dot{y}+10^{4} y+5 \times 10^{4} y^{3}=0
$$

and initial condition $\dot{y}(0)=200$. Fig. 25 a shows the decaying displacement and the envelope computed via Eq. (63). Fig. 25b shows the corresponding instantaneous frequency obtained from (66). The backbone and damping curves are given in Figs. 26a,b, respectively. As expected for a stiffening system, the natural frequency increases with the amplitude of excitation. Apart from a high-frequency modulation, the damping

\footnotetext{
${ }^{4}$ The results in Figs. 25-27 were obtained by Dr. Michael Feldman - the authors are very grateful for permission to use them.
} 

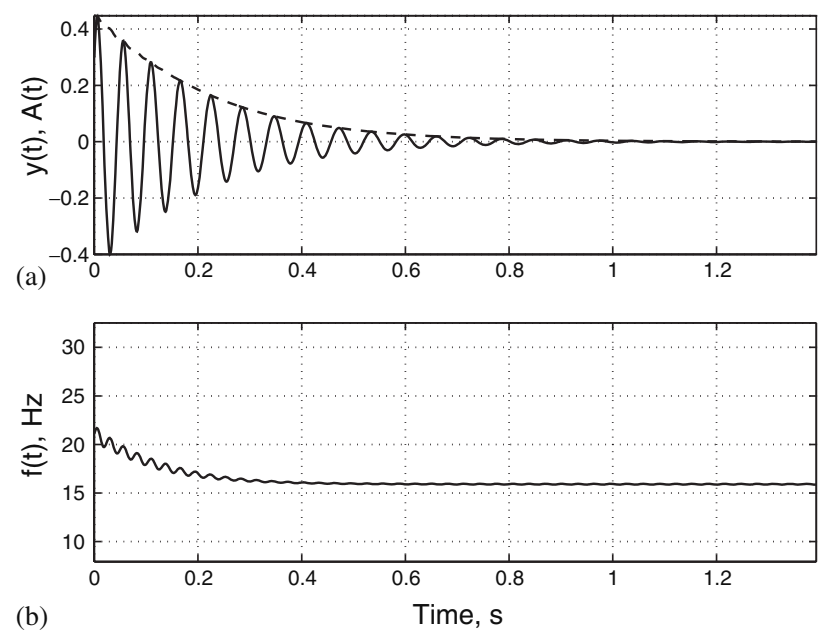

Fig. 25. Identification of cubic stiffness system: (a) impulse response; (b) envelope.
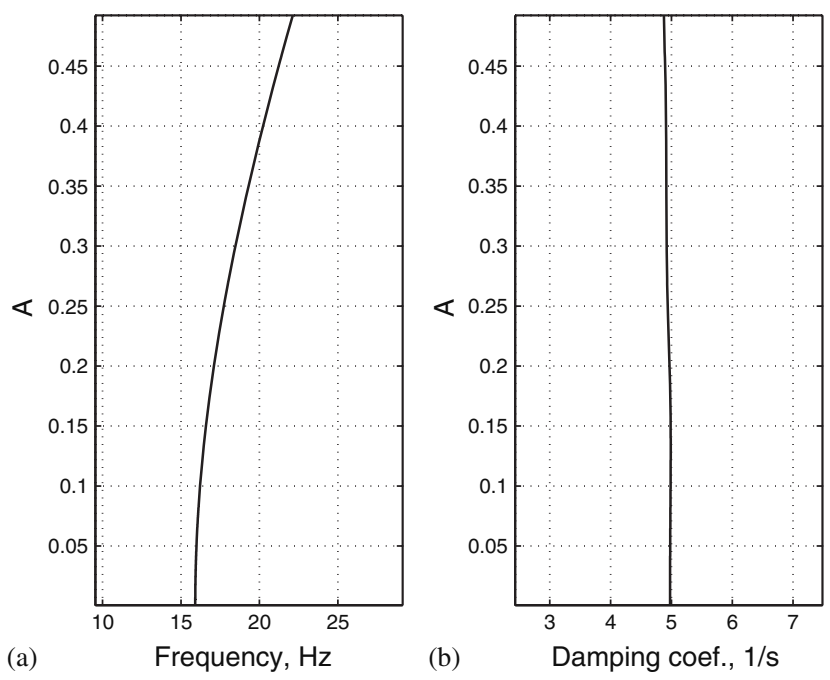

Fig. 26. Identification of cubic stiffness system: (a) backbone curve; (b) damping curve.

curve shows constant behaviour. Using Eqs. (76) and (77), the stiffness and damping curves can be obtained and these are shown in Figs. 27a,b.

Alternative approaches have been constructed to yield the same information, in particular the Wigner-Ville approach described by Feldman and Braun (1995 [277]), the method based on the Gabor transform in (Brancaleoni et al., 1992 [402]), the wavelet approach (Staszewski, 2000 [276]) and the time-domain Fourier filter output (Sainsbury and Ho, 2001 [403]). All of these approaches except FORCEVIB extract the information from the free decay response of the systems.

\subsubsection{Assessment}

The method described above is one of the most successful approach to tracking the varying nature of vibration of a large class of nonlinear systems. However, it is only truly suitable for monocomponent signals, i.e., those with a single frequency dominant. The extension to two-component signals is discussed in (Feldman, 1997 [175]). We mention that a method for the decomposition of signals with multiple components into a collection of monocomponents signals is proposed in Huang et al. (1998 [176]), which may extend the 

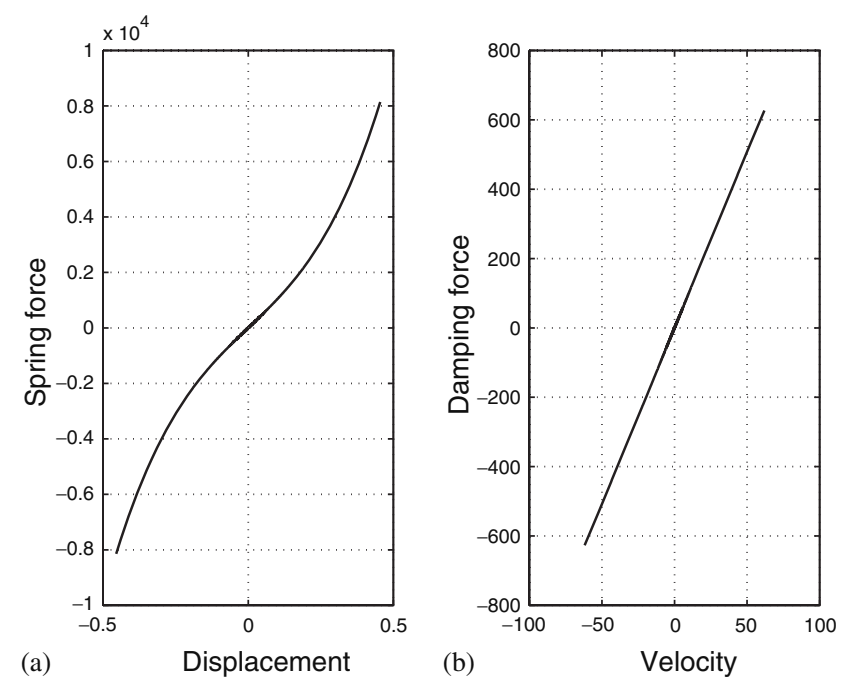

Fig. 27. Identification of cubic stiffness system: (a) stiffness characteristic; (b) damping characteristic.

applicability of the method. Another assumption implicit in the method is that the change in amplitude must be slow relative to the change in phase.

\subsection{The Volterra series and higher-order frequency response functions}

\subsubsection{Theory}

For a general linear system, the input-output map can be expressed by Duhamel's integral

$$
y(t)=\int_{-\infty}^{\infty} h(\tau) x(t-\tau) \mathrm{d} \tau .
$$

Eq. (79) is manifestly linear and therefore cannot hold for arbitrary nonlinear systems. However, it admits a generalisation. The extended form of Eq. (79) was obtained by Volterra (1959 [404]). It takes the form of an infinite series

$$
y(t)=y_{1}(t)+y_{2}(t)+y_{3}(t)+\cdots,
$$

where

$$
\begin{aligned}
& y_{1}(t)=\int_{-\infty}^{+\infty} h_{1}(\tau) x(t-\tau) \mathrm{d} \tau, \\
& y_{2}(t)=\int_{-\infty}^{+\infty} \int_{-\infty}^{+\infty} h_{2}\left(\tau_{1}, \tau_{2}\right) x\left(t-\tau_{1}\right) x\left(t-\tau_{2}\right) \mathrm{d} \tau_{1} \mathrm{~d} \tau_{2}, \\
& y_{3}(t)=\int_{-\infty}^{+\infty} \int_{-\infty}^{+\infty} \int_{-\infty}^{+\infty} h_{3}\left(\tau_{1}, \tau_{2}, \tau_{3}\right) x\left(t-\tau_{1}\right) x\left(t-\tau_{2}\right) x\left(t-\tau_{3}\right) \mathrm{d} \tau_{1} \mathrm{~d} \tau_{2} \mathrm{~d} \tau_{3} .
\end{aligned}
$$

The form of the general term is obvious from the above. The functions $h_{1}(\tau), h_{2}\left(\tau_{1}, \tau_{2}\right), h_{3}\left(\tau_{1}, \tau_{2}, \tau_{3}\right), \ldots$, $h_{n}\left(\tau_{1}, \ldots, \tau_{n}\right)$, etc. are generalisations of the linear impulse response function and are usually referred to as Volterra kernels. The use of the Volterra series in dynamics stems from the seminal paper of Barrett (1963 [405]) in which the series was applied to nonlinear differential equations for the first time. One can think of the series as a generalisation of the Taylor series from functions to functionals. The expression (79) simply represents the lowest-order truncation which is of course exact only for linear systems.

It can be shown (Schetzen, 1980 [188]) that the kernels can be considered to be symmetric without loss of generality; i.e., $h_{2}\left(\tau_{1}, \tau_{2}\right)=h_{2}\left(\tau_{2}, \tau_{1}\right)$, etc. 
There exists a dual frequency-domain representation for nonlinear systems. The HOFRFs or Volterra kernel transforms $H_{n}\left(\omega_{1}, \ldots, \omega_{n}\right), n=1, \ldots, \infty$ are defined as the multi-dimensional Fourier transforms of the kernels, i.e.,

$$
H_{n}\left(\omega_{1}, \ldots, \omega_{n}\right)=\int_{-\infty}^{+\infty} \cdots \int_{-\infty}^{+\infty} h_{n}\left(\tau_{1}, \ldots, \tau_{n}\right) \mathrm{e}^{-\mathrm{i}\left(\omega_{1} \tau_{1}+\cdots+\omega_{n} \tau_{n}\right)} \mathrm{d} \tau_{1} \ldots \mathrm{d} \tau_{n}
$$

It is a simple matter to show that symmetry of the kernels implies symmetry of the kernel transforms; so for example, $H_{2}\left(\omega_{1}, \omega_{2}\right)=H_{2}\left(\omega_{2}, \omega_{1}\right)$.

It is a straightforward matter to obtain the frequency-domain dual of expression (80)

$$
Y(\omega)=Y_{1}(\omega)+Y_{2}(\omega)+Y_{3}(\omega)+\cdots,
$$

where

$$
\begin{aligned}
& Y_{1}(\omega)=H_{1}(\omega) X(\omega), \\
& Y_{2}(\omega)=\frac{1}{2 \pi} \int_{-\infty}^{+\infty} H_{2}\left(\omega_{1}, \omega-\omega_{1}\right) X\left(\omega_{1}\right) X\left(\omega-\omega_{1}\right) \mathrm{d} \omega_{1} .
\end{aligned}
$$

One use of the Volterra series is the construction of analytic approximations to various quantities of interest in experimental structural analysis. Approximations to the FRFs of SDOF and MDOF systems with cubic nonlinearities and excited by Gaussian white noise can be found in (Worden and Manson, 1998 [406]; Worden and Manson, 1999 [407]). The approximations derived are of interest from the point of view that the FRFs constructed have all their poles in the upper-half of the complex frequency plane. This goes some way to explaining why the FRFs of randomly excited nonlinear systems appear to be invariant under the Hilbert transform. A Padé approximation to the coherence of the Duffing oscillator is constructed in (Worden and Manson, 2005 [408]), which shows excellent qualitative agreement with experiment.

The remainder of this section is concerned with the determination and interpretation of the HOFRFs.

Harmonic probing of the Volterra series: There are various methods of determining the HOFRFs for a system. If one has measured input and output time data, it is possible to evaluate the FRFs by carrying out many multi-dimensional fast Fourier transforms (FFTs) and averaging the results, in much the same way as one would evaluate a standard linear transfer function (Schetzen, 1980 [188]; Gifford, 1989 [190]; Gifford and Tomlinson, 1989 [409]). However, this approach requires that the input be a Gaussian white noise sequence; also, the computational burden of carrying out multi-dimensional FFTs makes evaluation of FRFs higher than second-order prohibitive by this method. Wray and Green (1994 [410]) proposed an interesting method of extracting the Volterra kernels for a system by fitting the time-domain response using a time-delay neural network (TDNN) and computing the kernels from the network weights. Alternatively, it is possible to estimate the HOFRFs efficiently by harmonic testing of a system as in Storer (1991 [191]). More recent work on identification of nonlinear systems via the Volterra kernels and kernel transforms can be found in (Khan and Vyas, 1999 [193]; Chatterjee and Vyas, 2003, 2004 [194,196]; Tawfiq and Vihn, 2003, 2004 [198,199]).

If one knows the equation of motion of a system, an alternative approach can be used which yields exact expressions for the HOFRFs. The method of harmonic probing was introduced by Bedrosian and Rice (1971 [168]) specifically for systems with continuous-time equations of motion. The method was extended to discrete-time systems by Billings and Tsang (1989b [170]). An alternative, recursive approach to probing is presented in (Peyton Jones and Billings, 1989 [411]).

In order to explain the harmonic probing procedure, it is necessary to determine how a system responds to a harmonic input in terms of its Volterra series.

First consider a periodic excitation composed of a single harmonic

$$
x(t)=\mathrm{e}^{\mathrm{i} \Omega t} .
$$

The spectral representation of this function follows immediately from the well-known representation of the $\delta$ function

$$
\delta(t)=\frac{1}{2 \pi} \int_{-\infty}^{\infty} \mathrm{e}^{\mathrm{i} \omega t} \mathrm{~d} \omega
$$


so that

$$
X(\omega)=2 \pi \delta(\omega-\Omega) .
$$

Substituting these expressions into Eqs. (81)-(83) and forming the total response as in (80) yields, up to third order,

$$
y(t)=H_{1}(\Omega) \mathrm{e}^{\mathrm{i} \Omega t}+H_{2}(\Omega, \Omega) \mathrm{e}^{\mathrm{i} 2 \Omega t}+H_{3}(\Omega, \Omega, \Omega) \mathrm{e}^{\mathrm{i} 3 \Omega t}+\cdots .
$$

This shows clearly that components in the output at multiples of the excitation frequency are expected, i.e., harmonics. The important point here is that the component in the output at the forcing frequency is $H_{1}(\Omega)$.

Probing the system with a single harmonic only yields information about the values of the FRFs on the diagonal line in the frequency spaces. In order to obtain further information, multi-frequency excitations must be used. With this in mind, consider the 'two-tone' input

$$
x(t)=\mathrm{e}^{\mathrm{i} \Omega_{1} t}+\mathrm{e}^{\mathrm{i} \Omega_{2} t}
$$

which has spectral representation

$$
X(\omega)=2 \pi \delta\left(\omega-\Omega_{1}\right)+2 \pi \delta\left(\omega-\Omega_{2}\right)
$$

substituting into (81)-(83) (or the frequency domain equivalents) and thence into (80) eventually yields, up to third order,

$$
\begin{aligned}
y(t)= & H_{1}\left(\Omega_{1}\right) \mathrm{e}^{\mathrm{i} \Omega_{1} t}+H_{1}\left(\Omega_{2}\right) \mathrm{e}^{\mathrm{i} \Omega_{2} t} \\
& +H_{2}\left(\Omega_{1}, \Omega_{1}\right) \mathrm{e}^{\mathrm{i} 2 \Omega_{1} t}+2 H_{2}\left(\Omega_{1}, \Omega_{2}\right) \mathrm{e}^{\mathrm{i}\left(\Omega_{1}+\Omega_{2}\right) t}+H_{2}\left(\Omega_{2}, \Omega_{2}\right) \mathrm{e}^{\mathrm{i} 2 \Omega_{2} t} \\
& +H_{3}\left(\Omega_{1}, \Omega_{1}, \Omega_{1}\right) \mathrm{e}^{\mathrm{i} 3 \Omega_{1} t}+3 H_{3}\left(\Omega_{1}, \Omega_{1}, \Omega_{2}\right) \mathrm{e}^{\mathrm{i}\left(2 \Omega_{1}+\Omega_{2}\right) t} \\
& +3 H_{3}\left(\Omega_{1}, \Omega_{2}, \Omega_{2}\right) \mathrm{e}^{\mathrm{i}\left(\Omega_{1}+2 \Omega_{2}\right) t}+H_{3}\left(\Omega_{2}, \Omega_{2}, \Omega_{2}\right) \mathrm{e}^{\mathrm{i} 3 \Omega_{2} t}+\cdots .
\end{aligned}
$$

The important thing to note here is that the amplitude of the component at the sum frequency for the excitation, i.e., at $\Omega_{1}+\Omega_{2}$, is twice the second-order FRF $H_{2}\left(\Omega_{1}, \Omega_{2}\right)$. In fact, if a general periodic excitation is used, i.e.,

$$
x(t)=\mathrm{e}^{\mathrm{i} \Omega_{1} t}+\cdots+\mathrm{e}^{\mathrm{i} \Omega_{n} t}
$$

it is not difficult to show that the amplitude of the output component at the frequency $\Omega_{1}+\cdots+\Omega_{n}$ is $n ! H_{n}\left(\Omega_{1}, \ldots, \Omega_{n}\right)$. This single fact is the basis of the harmonic probing algorithm. In order to find the secondorder FRF of a system for example, one substitutes the expressions for input (92) and general output (94) into the system's equation of motion and extracts the coefficient of $\mathrm{e}^{\mathrm{i}\left(\Omega_{1}+\Omega_{2}\right) t}$; this yields an algebraic expression for $\mathrm{H}_{2}$.

The procedure is best illustrated by choosing a concrete example. Consider the Duffing oscillator in Eq. (41) modified to include a term $k_{2} y^{2}$ on the left-hand side - an asymmetric Duffing oscillator. In order to find $H_{1}$, the probing expressions

$$
x(t)=x_{1}^{p}(t)=\mathrm{e}^{\mathrm{i} \Omega t}
$$

and,

$$
y(t)=y_{1}^{p}(t)=H_{1}(\Omega) \mathrm{e}^{\mathrm{i} \Omega t}
$$

are substituted into the asymmetric Duffing oscillator, the result being

$$
\left(-m \Omega^{2}+\mathrm{i} c \Omega+k\right) H_{1}(\Omega) \mathrm{e}^{\mathrm{i} \Omega t}+k_{2} H_{1}(\Omega)^{2} \mathrm{e}^{\mathrm{i} 2 \Omega t}+k_{3} H_{1}(\Omega)^{3} \mathrm{e}^{\mathrm{i} 3 \Omega t}=\mathrm{e}^{\mathrm{i} \Omega t}
$$

equating the coefficients of $\mathrm{e}^{\mathrm{i} \Omega t}$ on each side of this expression yields an equation for $H_{1}$

$$
\left(-m \Omega^{2}+\mathrm{i} c \Omega+k\right) H_{1}(\Omega)=1
$$

which is trivially solved, giving the expression

$$
H_{1}(\Omega)=\frac{1}{-m \Omega^{2}+\mathrm{i} c \Omega+k} .
$$


Evaluation of $\mathrm{H}_{2}$ is only a little more complicated. The probing expressions

$$
x(t)=x_{2}^{p}(t)=\mathrm{e}^{\mathrm{i} \Omega_{1} t}+\mathrm{e}^{\mathrm{i} \Omega_{2} t}
$$

and

$$
y(t)=y_{2}^{p}(t)=H_{1}\left(\Omega_{1}\right) \mathrm{e}^{\mathrm{i} \Omega_{1} t}+H_{1}\left(\Omega_{2}\right) \mathrm{e}^{\mathrm{i} \Omega_{2} t}+2 H_{2}\left(\Omega_{1}, \Omega_{2}\right) \mathrm{e}^{\mathrm{i}\left(\Omega_{1}+\Omega_{2}\right) t}
$$

are used. Note that in passing from the general output (94) to the probing expression (102), all second-order terms except that at the sum frequency have been deleted. This is a very useful simplification and is allowed because no combination of the missing terms can produce a component at the sum frequency and therefore they cannot appear in the final expression for $\mathrm{H}_{2}$. Substituting (101) and (102) into the asymmetric form of (41), and extracting the coefficients of $\mathrm{e}^{\mathrm{i}\left(\Omega_{1}+\Omega_{2}\right) t}$ yields

$$
H_{2}\left(\Omega_{1}, \Omega_{2}\right)=-\frac{k_{2}}{2} H_{1}\left(\Omega_{1}\right) H_{1}\left(\Omega_{2}\right) H_{1}\left(\Omega_{1}+\Omega_{2}\right) .
$$

Note that the constant $k_{2}$ multiplies the whole expression for $\mathrm{H}_{2}$, so that if the square-law term is absent from the equation of motion, $\mathrm{H}_{2}$ vanishes. This reflects a quite general property of the Volterra series; if all nonlinear terms in the equation of motion for a system are odd powers of $x$ or $y$, then the associated Volterra series has no even order kernels. As a consequence it will possess no even order kernel transforms.

In order to obtain $H_{3}$, the required probing expressions are

$$
x(t)=x_{3}^{p}(t)=\mathrm{e}^{\mathrm{i} \Omega_{1} t}+\mathrm{e}^{\mathrm{i} \Omega_{2} t}+\mathrm{e}^{\mathrm{i} \Omega_{3} t}
$$

and

$$
\begin{aligned}
y(t)= & y_{3}^{p}(t)=H_{1}\left(\Omega_{1}\right) \mathrm{e}^{\mathrm{i} \Omega_{1} t}+H_{1}\left(\Omega_{2}\right) \mathrm{e}^{\mathrm{i} \Omega_{2} t}+H_{1}\left(\Omega_{3}\right) \mathrm{e}^{\mathrm{i} \Omega_{3} t} \\
& +2 H_{2}\left(\Omega_{1}, \Omega_{2}\right) \mathrm{e}^{\mathrm{i}\left(\Omega_{1}+\Omega_{2}\right) t}+2 H_{2}\left(\Omega_{1}, \Omega_{3}\right) \mathrm{e}^{\mathrm{i}\left(\Omega_{1}+\Omega_{3}\right) t}+2 H_{2}\left(\Omega_{2}, \Omega_{3}\right) \mathrm{e}^{\mathrm{i}\left(\Omega_{2}+\Omega_{3}\right) t} \\
& +6 H_{3}\left(\Omega_{1}, \Omega_{2}, \Omega_{3}\right) \mathrm{e}^{\mathrm{i}\left(\Omega_{1}+\Omega_{2}+\Omega_{3}\right) t},
\end{aligned}
$$

which are sufficiently general to obtain $H_{3}$ for any system. Substituting into the asymmetric Duffing equation and extracting the coefficient of $\mathrm{e}^{\mathrm{i}\left(\Omega_{1}+\Omega_{2}+\Omega_{3}\right) t}$ yields

$$
\begin{aligned}
& H_{3}\left(\Omega_{1}, \Omega_{2}, \Omega_{3}\right)=-\frac{1}{6} H_{1}\left(\Omega_{1}+\Omega_{2}+\Omega_{3}\right) . \\
& \left\{4 k_{2}\left(H_{1}\left(\Omega_{1}\right) H_{2}\left(\Omega_{2}, \Omega_{3}\right)+H_{1}\left(\Omega_{2}\right) H_{2}\left(\Omega_{3}, \Omega_{1}\right)+H_{1}\left(\Omega_{3}\right) H_{2}\left(\Omega_{1}, \Omega_{2}\right)\right)+k_{3} H_{1}\left(\Omega_{1}\right) H_{1}\left(\Omega_{2}\right) H_{1}\left(\Omega_{3}\right)\right\} .
\end{aligned}
$$

It is a general property of systems that all HOFRFs can be expressed in terms of $H_{1}$ for the system. The exact form of the expression will of course depend on the particular system.

The harmonic probing algorithm is established above for continuous-time systems, i.e., those whose evolution is governed by differential equations of motion. The NARMAX models discussed earlier are difference equations so the probing algorithm requires a little modification as in (Billings and Tsang, 1989b [170]).

The method of Wray and Green described earlier which extracted Volterra kernels from TDNNs (Wray and Green, 1994 [410]), was inextendible to the NARX case. However, Chance et al. (1998 [412]) showed that it was possible to extract kernel transforms or HOFRFs by fitting multi-layer perceptron neural networks and then using harmonic probing. Ideas from machine learning theory have proved useful in other respects for Volterra series approximation. Kernel methods and ideas based on reproducing kernel Hilbert spaces have proved fruitful (Dodd and Harris, 2002 [413]; Dodd and Harrison, 2002a,b [414,415]). One particular result of interest is a method for estimating the entire Volterra series without truncation (Wan, 2003 [416]).

Validation and interpretation of the higher-order frequency response functions: In order to justify studying the HOFRFs it is necessary to show that they contain useful information about whatever system is under examination. In fact, as time and frequency-domain representations are completely equivalent, the HOFRFs contain all system information; in this section it is demonstrated that important facts can be conveyed in a very direct and visible way. 
In order to demonstrate accurate FRFs from a NARMAX model, the following numerical simulation was carried out. A fourth-order Runge-Kutta scheme (Press et al., 1986 [417]) was used to obtain the response of the asymmetric Duffing oscillator under excitation by a Gaussian noise sequence $x(t)$ with rms 10.0 and frequency range 0 to $90 \mathrm{~Hz}$. The coefficient values adopted were: $m=1, c=20, k=10^{4}, k_{2}=10^{7}, k_{3}=5.10^{9}$. This system has a resonant frequency of $\omega_{r}=\sqrt{k / m}=99 \mathrm{rad} / \mathrm{s}$ or $f_{r}=\omega_{r} / 2 \pi=15.75 \mathrm{~Hz}$. The data was generated with a sampling interval of $0.005 \mathrm{~s}$, giving a Nyquist frequency of $100 \mathrm{~Hz}$.

A NARMAX model was fitted to 1000 points of the resulting discrete $x$ and $y$ data using least-squares parameter estimation and validation methods described in the previous section. The result was

$$
\begin{aligned}
y_{i}= & 1.6696 y_{i-1}-0.90348 y_{i-2} \\
& -2.1830 \times 10^{2} y_{i-1}^{2}-1.0665 \times 10^{5} y_{i-1}^{3} \\
& +3.0027 \times 10^{-6} x_{i}+1.8040 \times 10^{-5} x_{i-1} \\
& +2.7676 \times 10^{-6} x_{i-2} .
\end{aligned}
$$

The fitted model was then used to generate the HOFRFs $H_{1}, H_{2}$ and $H_{3}$ by the method of harmonic probing. As the exact results could also be obtained by harmonic probing of equation for the Duffing oscillator, direct comparisons could be made. In all cases, the exact FRFs are given with the frequency scale in $\mathrm{Hz}$; the FRFs for the discrete model are given with corresponding normalised frequency scales $f_{n}=f / f_{s}$ where $f_{s}$ is the sampling frequency, the Nyquist frequency is 0.5 in these units. The HOFRFs are calculated and plotted using the FREP package of Tsang and Billings (1988 [418]).

Fig. 28 shows a comparison between the exact $H_{1}$ and that obtained from the model, the agreement is excellent. However, an important point must be raised here. $H_{1}$ for the discrete system is only plotted up to the Nyquist frequency in Figs. 28c and d because it simply repeats beyond this point and is therefore meaningless.

The comparison between the exact $H_{2}$ and that from the NARMAX model is given in Fig. 29 using contour maps for the functions. Note that because $H_{2}$ contains factors $H_{1}\left(2 \pi f_{1}\right)$ and $H_{2}\left(2 \pi f_{2}\right)$ it would be meaningless to plot it outside the ranges corresponding to $f_{1} \leqslant 100, f_{2} \leqslant 100$. Further, $H_{2}$ also contains a factor $H_{1}\left(2 \pi\left(f_{1}+f_{2}\right)\right)$ so that the plots should not extend past the area specified by $f_{1}+f_{2} \leqslant 100$. The Nyquist region is that bounded by the solid lines in Fig. 29.

The comparison between the $H_{3}$ functions also shows good agreement, they are not shown here. Note that the whole $\mathrm{H}_{3}$ surface cannot be plotted as it exists as a three-dimensional manifold embedded in a fourdimensional space over the $\left(\omega_{1}, \omega_{2}, \omega_{3}\right)$ 'plane'. However, one can plot two-dimensional submanifolds of $H_{3}$, and this is the approach which is usually adopted.

As an aside, the first-order FRF has been used in system identification for some time; the well-established technique of modal analysis (Ewins, 2000 [11]) is based on the extraction of linear system parameters by curve-fitting to the FRF. Gifford (1989 [190]) showed that the technique extends naturally to nonlinear systems; nonlinear parameters are extracted by fitting surfaces or hypersurfaces to the HOFRFs. A remarkable observation is that for a nonlinear system, the nonlinear parameters are much easier to obtain from the HOFRFs than the linear parameters are from $H_{1}$ because, for instance, the quadratic stiffness coefficient $k_{2}$ enters as a linear multiplier in Eq. (103); this is also the case for the cubic coefficient as demonstrated by Eq. (106). This work was further extended by Storer (Storer and Tomlinson, 1991 [419]; Storer, 1991 [191]), who demonstrated that it is sufficient to curve-fit to the parts of the FRFs above the diagonal frequency subspaces. This allowed a significantly simpler experimental procedure based on harmonic testing. The HOFRFs can also be obtained by impulse testing (Liu et al., 1987 [420]). Finally, returning to the subject of this section proper, Tsang and Billings (1992 [421]) have demonstrated a general method of using the HOFRFs obtained from NARMAX models to pass to a continuous-time model.

Having established some confidence in their reliability, the interpretation of the HOFRFs can be discussed. The Duffing oscillator system (107) serves well as an illustration. The magnitude and phase of expression (107) for $H_{1}(\omega)=H_{1}(2 \pi f)$ is given in Figs. 28a and b on the frequency interval $0-100 \mathrm{~Hz}$. The interpretation of these figures, traditionally given together and universally called the Bode plot, is well known; the peak in the magnitude at $f=f_{r}=15.75 \mathrm{~Hz}$ shows that for this frequency of excitation the amplitude of the linear part of 

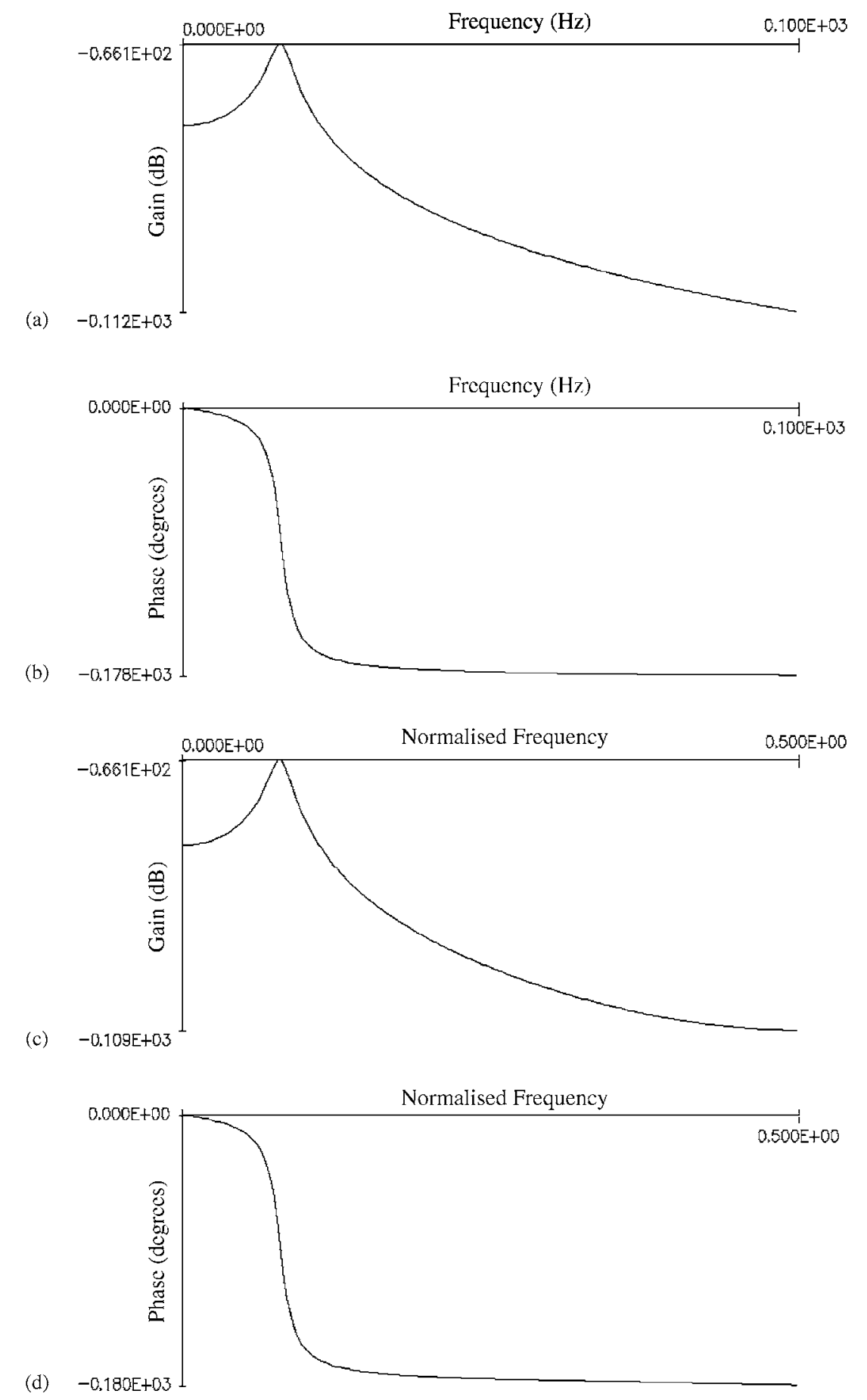

Fig. 28. Estimated and exact $H_{1}$ functions for the Duffing oscillator: (a)-(b) exact; (c)-(d) estimation.

the response $y_{1}(t)$ is a maximum. The Bode plot thus allows the immediate identification of those excitation frequencies at which the vibration level of the system is likely to be high.

Interpretation of the second-order FRF is also straightforward. The magnitude and phase of $H_{2}$ for the Duffing system above are given in Figs. $29 \mathrm{a}$ and $\mathrm{b}$ as contour maps over the $\left(f_{1}, f_{2}\right)=\left(\frac{\omega_{1}}{2 \pi}, \frac{\omega_{2}}{2 \pi}\right)$ plane. The frequency ranges for the plot are the same as for $H_{1}$ in Fig. 28. A number of ridges are observed. These are in 

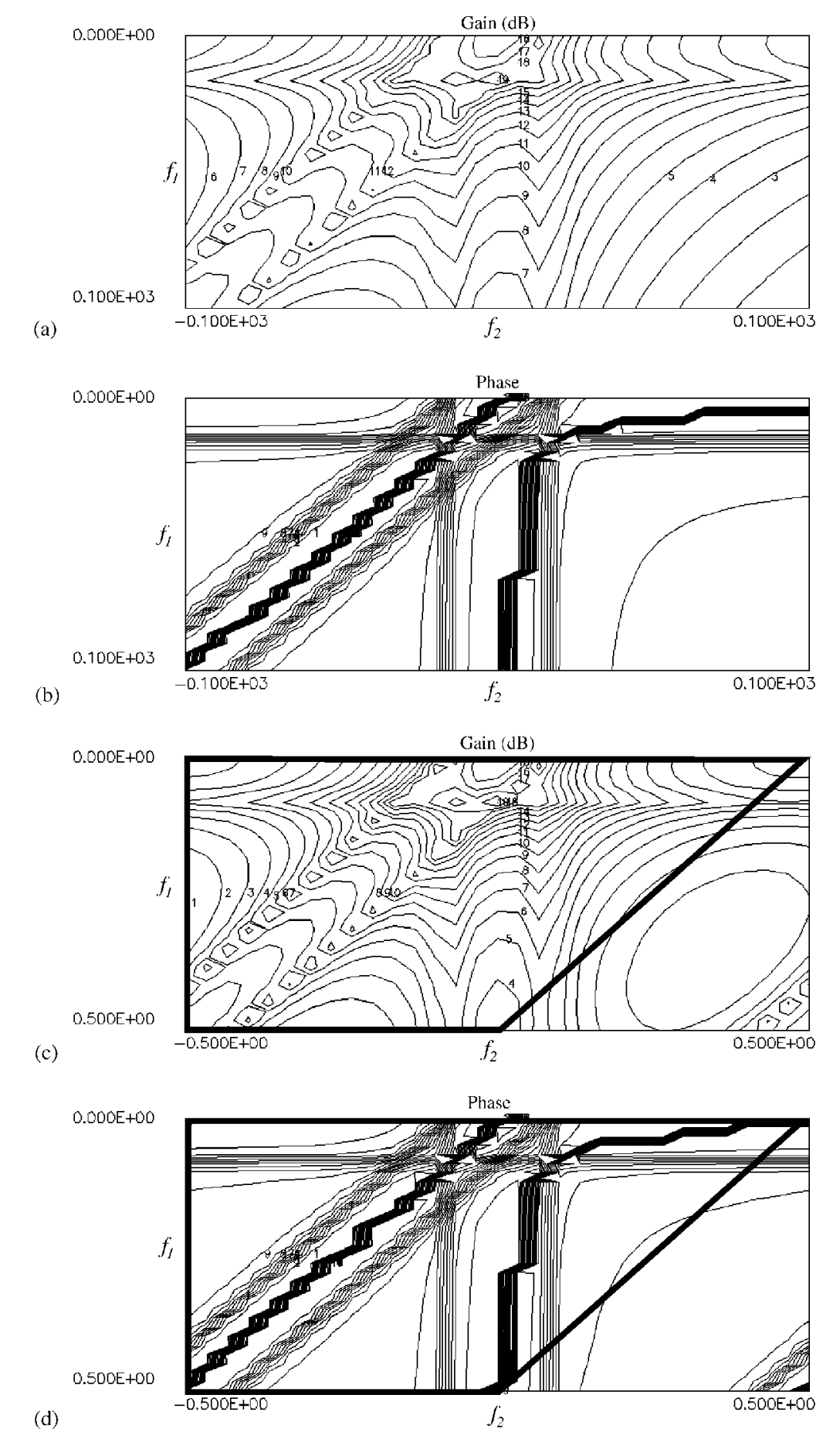

Fig. 29. Estimated and exact $\mathrm{H}_{2}$ functions for the Duffing oscillator: (a)-(b) exact; (c)-(d) estimation.

direct correspondence with the peak in $H_{1}$ as follows. According to Eq. (103), $H_{2}$ is a constant multiple of $H_{1}\left(\omega_{1}\right) H_{1}\left(\omega_{2}\right) H_{1}\left(\omega_{1}+\omega_{2}\right)$. As a consequence $H_{2}$ possesses local maxima at positions where the $H_{1}$ factors have local maxima. Consequently there are two ridges in the $H_{2}$ surface corresponding to the lines $\omega_{1}=$ $\omega_{r}=2 \pi f_{r}$ and $\omega_{2}=\omega_{r}$. These are along lines parallel to the frequency axes. In addition, $H_{2}$ has local maxima generated by the $H_{1}\left(\omega_{1}+\omega_{2}\right)$ factor along the line $\omega_{1}+\omega_{2}=\omega_{r}$. This ridge has an important consequence; it 
indicates that one can expect a maximum in the second-order output $y_{2}(t)$ if the system is excited by two sinusoids whose sum frequency is the linear resonant frequency, i.e., a combination resonance (Nayfeh and Mook, 1979 [28]). This shows clearly why estimation of a transfer function by linear methods is inadequate for nonlinear systems; such a transfer function would usually indicate a maximum in the output for a harmonic excitation close to the linear resonant frequency.However, it would fail to predict that one could excite a large nonlinear component in the output by exciting at $\omega=\omega_{r} / 2$; this is a consequence of the trivial decomposition $2 \mathrm{e}^{\mathrm{i} \omega_{r} / 2 t}=\mathrm{e}^{\mathrm{i} \omega_{r} / 2 t}+\mathrm{e}^{\mathrm{i} \omega_{r} / 2 t}$ which means that the signal can be regarded as a two-tone input with a sum frequency at the linear resonance $\omega_{r}$. The importance of the second-order FRF is now clear. It reveals those pairs of excitation frequencies which will conspire to produce large levels of vibration as a result of second-order nonlinear effects.

The interpretation of $\mathrm{H}_{3}$ for the system is very similar, analogous ridges showing third-order interactions between frequencies are observed.

\subsubsection{Assessment}

The Volterra series is an appealing means of establishing a non-parametric (black-box) model of a nonlinear input-output process. The kernel transforms - the HOFRFs - provide an attractive means of identifying and interpreting interactions between input frequencies and give a visualisation equivalent to the Bode plot for a linear system. One disadvantage of this visualisation is that the HOFRFs are objects with higher dimension than 3 for kernel orders higher than 3 and therefore one can only inspect lower-dimensional projections. Computation can be intensive in terms of both time and data requirements if a stepped-sine approach or correlation approach is adopted; however, the HOFRFs can be computed quickly by harmonic probing of NARX (or other parametric) models. The main limitation of the Volterra approach is the existence and convergence of the series. Many nonlinearities of practical interest have discontinuous or non-smooth nonlinearities and the corresponding systems do not strictly have a Volterra representation. Also, as the Volterra series is a functional Taylor series, it is single-valued and loses validity if the system being modeled is in the vicinity of a bifurcation. A related problem is that the radius of the convergence of the series may be restricted or that low-order truncations may not be accurate.

\subsection{The reverse path method}

\subsubsection{Theory}

Frequency-domain modal parameter estimation techniques are extensively used to identify the properties of linear systems. They extract modal parameters from $H_{1}$ and $H_{2}$ estimated FRFs (Ewins, 2000 [11])

$$
H_{1}(\omega)=\frac{S_{y x}(\omega)}{S_{x x}(\omega)}, \quad H_{2}(\omega)=\frac{S_{y y}(\omega)}{S_{y x}(\omega)},
$$

where $S_{y y}(\omega), S_{x x}(\omega)$ and $S_{y x}(\omega)$ contain the PSD of the response (e.g., acceleration signal), the PSD of the applied force and the cross-PSD between the response and the applied force, respectively. In the presence of nonlinear forces, the $H_{1}$ and $H_{2}$ estimators cannot be used because nonlinearities corrupt the underlying linear characteristics of the response.

Reverse path spectral methods were therefore introduced to accommodate the presence of nonlinearity. The description of the reverse path concept is limited to SDOF systems in this section (Rice and Fitzpatrick, 1988 [213]; Bendat, 1990 [216]). The extension to MDOF systems is discussed in (Rice and Fitzpatrick, 1991b [221]), whereas an 'enhanced' reverse path spectral method, the CRP method (Richards and Singh, 1998 [222]), also suitable for identification of MDOF systems, is presented in Section 7.1.

The method is best described via a simple illustration. Therefore, consider once again the symmetric Duffing equation

$$
m \ddot{y}+c \dot{y}+k y+k_{3} y^{3}=x(t) .
$$

Taking the Fourier transform $\mathscr{F}[\bullet]$ of this equation gives

$$
B(\omega) Y(\omega)+A(\omega) Z(\omega)=X(\omega),
$$




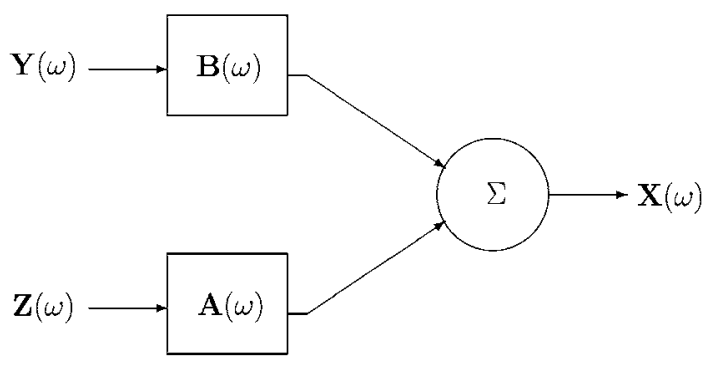

Fig. 30. A two-input-single-output reverse path model.

where $B(\omega)=-\omega^{2} m+\mathrm{i} \omega c+k, Y(\omega)=\mathscr{F}[y(t)]$ and $X(\omega)=\mathscr{F}[x(t)]$ are the objects usually observed for a linear system $\left[B(\omega)=H(\omega)^{-1}\right.$ is the linear dynamic stiffness matrix]. In this case, $A(\omega)=k_{3}$ and $Z(\omega)=\mathscr{F}\left[y^{3}(t)\right]$. Note that the latter object could be expressed as a rather messy convolution involving $Y(\omega)$.

By exchanging the roles of input and output, one can regard Eq. (110) as representing a two-input-singleoutput system as depicted in Fig. 30. Multiplying Eq. (110) by $Y(\omega)$ and taking expectation gives

$$
B(\omega) S_{y y}(\omega)+A(\omega) S_{y z}(\omega)=S_{y x}(\omega) .
$$

Similarly, multiplying (110) by $Z(\omega)$ and taking expectation gives

$$
B(\omega) S_{z y}(\omega)+A(\omega) S_{z z}(\omega)=S_{z x}(\omega) .
$$

For each frequency, Eqs. (111) and (112) provide two simultaneous equations for the unknowns $B(\omega)$ and $A(\omega)$

$$
\left.\left.\begin{array}{ll}
S_{y y}(\omega) & S_{y z}(\omega) \\
S_{z y}(\omega) & S_{z z}(\omega)
\end{array}\right)\left(\begin{array}{c}
B(\omega) \\
A(\omega)
\end{array}\right)=\begin{array}{l}
S_{y x}(\omega) \\
S_{z x}(\omega)
\end{array}\right) .
$$

Note that as $A(\omega)=k_{3}$, it should simply be a real constant. However, as a result of the signal processing it will be derived as a complex spectrum. If the analysis is carried out accurately though, the quantity will have small variation with frequency and the imaginary part will generally be orders of magnitude smaller than the real part. The best estimate of $k_{3}$ would usually be obtained by averaging $A(\omega)$ over frequency.

\subsubsection{Assessment}

The reverse path formulation can solve nonlinear problems that can be modeled by means of a nonlinear differential or integrodifferential equation of motion in many fields because it is valid for random data with arbitrary probability, correlation and spectral properties (Bendat, 1998 [108]). In addition, identification is carried out through simple mathematical operations, and the results are in a form that is convenient to interpret.

A possible drawback of the method for MDOF systems (which has been removed in the CRP method) is that the excitation must be applied at the location of the nonlinearity in order to identify its coefficient. This issue is discussed in detail in (Richards and Singh, 1998 [222]).

\section{Parameter estimation in the presence of nonlinearity: recent methods}

In this section, several recent methods for parameter estimation in the presence of nonlinearity are described. These methods show promise for identification of more complex nonlinear structures because they are inherently capable of dealing with MDOF systems. Numerical and/or experimental examples are presented to illustrate their fundamental concepts but also their assets and limitations.

\subsection{The conditioned reverse path method}

To address the problem of the location of the external force inherent in the reverse path method, the CRP method developed in (Richards and Singh, 1998 [222]) employs spectral conditioning techniques to 
remove the effects of nonlinearities before computing the FRFs of the underlying linear system contained in the dynamic compliance matrix $\mathbf{H}(\omega)=\mathbf{B}^{-1}(\omega)$. The key idea of the formulation is the separation of the nonlinear part of the system response from the linear part and the construction of uncorrelated response components in the frequency domain. The nonlinear coefficients are estimated during the second phase of the method.

\subsubsection{Theory}

Estimation of the underlying system properties: The vibrations of a nonlinear system are governed by the following equation:

$$
\mathbf{M} \ddot{\mathbf{y}}(t)+\mathbf{C} \dot{\mathbf{y}}(t)+\mathbf{K} \mathbf{y}(t)+\sum_{j=1}^{n} \mathbf{A}_{j} \mathbf{z}_{j}(t)=\mathbf{x}(t),
$$

where $\mathbf{M}, \mathbf{C}$ and $\mathbf{K}$ are the structural matrices; $\mathbf{y}(t)$ is the vector of displacement coordinates; $\mathbf{z}_{j}(t)$ is a nonlinear function vector; $\mathbf{A}_{j}$ contains the coefficients of the term $\mathbf{z}_{j}(t) ; \mathbf{x}(t)$ is the applied force vector. For example, in the case of a grounded cubic stiffness at the $i$ th DOF, the nonlinear function vector is

$$
\mathbf{z}(t)=\left[\begin{array}{llllll}
0 & \ldots & y_{i}(t)^{3} & \ldots & 0
\end{array}\right]^{\mathrm{T}} .
$$

In the frequency domain, Eq. (114) becomes

$$
\mathbf{B}(\omega) \mathbf{Y}(\omega)+\sum_{j=1}^{n} \mathbf{A}_{j} \mathbf{Z}_{j}(\omega)=\mathbf{X}(\omega)
$$

where $\mathbf{Y}(\omega), \mathbf{Z}_{j}(\omega)$ and $\mathbf{X}(\omega)$ are the Fourier transform of $\mathbf{y}(t), \mathbf{z}_{j}(t)$ and $\mathbf{x}(t)$, respectively; $\mathbf{B}(\omega)=-\omega^{2} \mathbf{M}+$ $\mathrm{i} \omega \mathbf{C}+\mathbf{K}$ is the linear dynamic stiffness matrix.

Without loss of generality, let us assume that a single nonlinear term $\mathbf{Z}_{1}$ is present. The spectrum of the measured responses $\mathbf{Y}$ can be decomposed into a component $\mathbf{Y}_{(+1)}$ correlated with the spectrum of the nonlinear vector $\mathbf{Z}_{1}$ through a frequency response matrix $\mathbf{L}_{1 Y}$, and a component $\mathbf{Y}_{(-1)}$ uncorrelated with the spectrum of the nonlinear vector; i.e., $\mathbf{Y}=\mathbf{Y}_{(+1)}+\mathbf{Y}_{(-1)}$. In what follows, the minus (plus) sign signifies uncorrelated (correlated) with. Likewise, the spectrum of the external force $\mathbf{X}$ can be decomposed into a component $\mathbf{X}_{(+1)}$ correlated with the spectrum of the nonlinear vector $\mathbf{Z}_{1}$ through a frequency response matrix $\mathbf{L}_{1 X}$, and a component $\mathbf{X}_{(-1)}$ uncorrelated with the spectrum of the nonlinear vector; i.e., $\mathbf{X}=\mathbf{X}_{(+1)}+\mathbf{X}_{(-1)}$. Since both vectors $\mathbf{Y}_{(-1)}$ and $\mathbf{X}_{(-1)}$ are uncorrelated with the nonlinear vector, they correspond to the response of the underlying linear system and the force applied to this system, respectively; as a result, the path between them is the linear dynamic stiffness matrix $\mathbf{B}$

$$
\mathbf{X}_{(-1)}(\omega)=\mathbf{B}(\omega) \mathbf{Y}_{(-1)}(\omega) \text {. }
$$

The whole procedure is presented in diagram form in Fig. 31.

The generalisation to multiple nonlinearities is straightforward. In this case, the spectra of the response and the force need to be uncorrelated with all $n$ nonlinear function vectors

$$
\left\{\begin{array}{l}
\mathbf{Y}_{(-1: n)}=\mathbf{Y}-\sum_{j=1}^{n} \mathbf{Y}_{(+j)}=\mathbf{Y}-\sum_{j=1}^{n} \mathbf{L}_{j Y} \mathbf{Z}_{j(-1: j-1)}, \\
\mathbf{X}_{(-1: n)}=\mathbf{X}-\sum_{j=1}^{n} \mathbf{L}_{j X} \mathbf{Z}_{j(-1: j-1)}
\end{array}\right.
$$

where $\mathbf{Y}_{(-1: n)}$ and $\mathbf{X}_{(-1: n)}$ are both uncorrelated with the nonlinear function vectors; the path between them is the linear dynamic stiffness matrix $\mathbf{B}$

$$
\mathbf{X}_{(-1: n)}(\omega)=\mathbf{B}(\omega) \mathbf{Y}_{(-1: n)}(\omega)
$$

By transposing Eq. (119), premultiplying by the complex conjugate of $\mathbf{Y}$ (i.e., $\mathbf{Y}^{*}$ ) taking the expectation $E[\bullet]$ and multiplying by $2 / T$, the underlying linear system can be identified without corruption from the 


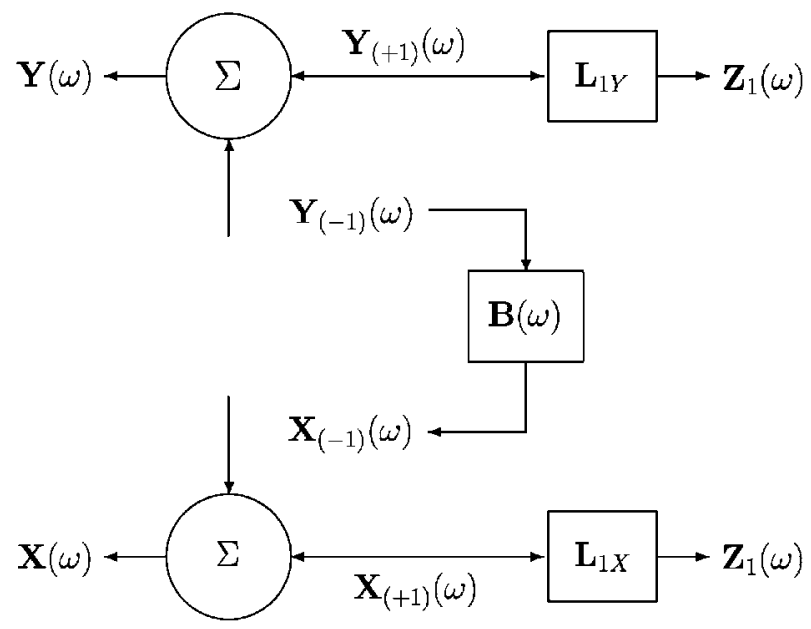

Fig. 31. Decomposition of the force and response spectra in the presence of a single nonlinearity.

nonlinear terms

$$
\begin{aligned}
\mathbf{S}_{y x(-1: n)} & =\frac{2}{T} E\left[\mathbf{Y}^{*} \mathbf{X}_{(-1: n)}^{\mathrm{T}}\right]=\frac{2}{T} E\left[\mathbf{Y}^{*}\left(\mathbf{B} \mathbf{Y}_{(-1: n)}\right)^{\mathrm{T}}\right] \\
& =\frac{2}{T} E\left[\mathbf{Y}^{*} \mathbf{Y}_{(-1: n)}^{\mathrm{T}} \mathbf{B}^{\mathrm{T}}\right]=\mathbf{S}_{y y(-1: n)} \mathbf{B}^{\mathrm{T}},
\end{aligned}
$$

where $\mathbf{S}_{y x(-1: n)}$ and $\mathbf{S}_{y y(-1: n)}$ are conditioned PSD matrices. Calculation of these matrices is laborious and involves a recursive algorithm. For the sake of conciseness, only the final formulae are given herein. In (Bendat and Piersol, 2000 [109]), it is shown that

$$
\mathbf{S}_{i j(-1: r)}=\mathbf{S}_{i j(-1: r-1)}-\mathbf{S}_{i r(-1: r-1)} \mathbf{L}_{r j}^{\mathrm{T}},
$$

where

$$
\mathbf{L}_{r j}^{\mathrm{T}}=\mathbf{S}_{r r(-1: r-1)}^{-1} \mathbf{S}_{r j(-1: r-1)} .
$$

It follows from Eq. (120) that the dynamic compliance matrix $\mathbf{H}$ which contains the FRFs of the underlying linear system takes the form

$$
H_{c 2}: \mathbf{H}^{\mathrm{T}}=\mathbf{S}_{y x(-1: n)}^{-1} \mathbf{S}_{y y(-1: n)} .
$$

This expression is known as the conditioned $H_{c 2}$ estimate. If relation (119) is multiplied by the complex conjugate of $\mathbf{X}$ instead of $\mathbf{Y}$, the conditioned $H_{c 1}$ estimate is obtained

$$
H_{c 1}: \mathbf{H}^{\mathrm{T}}=\mathbf{S}_{x x(-1: n)}^{-1} \mathbf{S}_{x y(-1: n)} .
$$

When FRFs of linear systems are estimated, $H_{1}$ always produces better estimates when there is measurement noise on the outputs, and $\mathrm{H}_{2}$ produces better estimates when the noise is on the input measurements. Intuition may lead us to expect the $H_{c 2}$ estimate to perform better than the $H_{c 1}$ estimate in the presence of uncorrelated noise only in the excitation. Likewise, the $H_{c 1}$ estimate is expected to perform better than the $H_{c 2}$ estimate in the presence of uncorrelated noise only in the response. However, experience shows that the $H_{c 2}$ estimate gives more accurate estimation of the FRFs of the underlying linear system in both situations. This may be a result of the conditioning required to calculate these estimates.

Estimation of the nonlinear coefficients: Once the linear dynamic compliance $\mathbf{H}$ has been computed by solving Eq. (123) or (124) at each frequency, the nonlinear coefficients $\mathbf{A}_{j}$ can be estimated. By applying to Eq. (116) the same procedure as the one used for obtaining Eq. (120) from Eq. (119), the following 
relationship is obtained

$$
\mathbf{S}_{i x(-1: i-1)}=\mathbf{S}_{i y(-1: i-1)} \mathbf{B}^{\mathrm{T}}+\sum_{j=1}^{n} \mathbf{S}_{i j(-1: i-1)} \mathbf{A}_{j}^{\mathrm{T}} .
$$

It should be noted that $\mathbf{S}_{i j(-1: i-1)}=E\left[\mathbf{Z}_{i(-1: i-1)}^{*} \mathbf{Z}_{j}^{\mathrm{T}}\right]=\mathbf{0}$ for $j<i$ since $\mathbf{Z}_{i(-1: i-1)}^{*}$ is uncorrelated with the spectrum of the nonlinear function vectors $\mathbf{Z}_{1}$ through $\mathbf{Z}_{i-1}$. If Eq. (125) is premultiplied by $\mathbf{S}_{i i(-1: i-1)}^{-1}$, the first term in the summation is $\mathbf{A}_{i}^{\mathrm{T}}$. Eq. (125) is then transformed into

$$
\left.\mathbf{A}_{i}^{\mathrm{T}}=\mathbf{S}_{i i(-1: i-1)}^{-1} \quad \mathbf{S}_{i x(-1: i-1)}-\mathbf{S}_{i y(-1: i-1)} \mathbf{B}^{\mathrm{T}}-\sum_{j=i+1}^{n} \mathbf{S}_{i j(-1: i-1)} \mathbf{A}_{j}^{\mathrm{T}}\right) .
$$

Because the expression of the linear dynamic compliance has been computed, Eq. (126) is rewritten in a more suitable form

$$
\left.\mathbf{A}_{i}^{\mathrm{T}} \mathbf{H}^{\mathrm{T}}=\mathbf{S}_{i i(-1: i-1)}^{-1} \quad \mathbf{S}_{i x(-1: i-1)} \mathbf{H}^{\mathrm{T}}-\mathbf{S}_{i y(-1: i-1)}-\sum_{j=i+1}^{n} \mathbf{S}_{i j(-1: i-1)} \mathbf{A}_{j}^{\mathrm{T}} \mathbf{H}^{\mathrm{T}}\right) .
$$

The identification process starts with the computation of $\mathbf{A}_{n}$ working backwards to $\mathbf{A}_{1}$. As for the reverse path method in Section 6.6, the nonlinear coefficients are imaginary and frequency dependent. The imaginary parts, without any physical meaning, should be negligible when compared to the real parts. On the other hand, by performing a spectral mean, the actual value of the coefficients should be retrieved.

Coherence functions: As explained in Section 4.1, the ordinary coherence function can be used to detect any departure from linearity or to detect the presence of uncorrelated noise on one or both of the excitation and response signals.

For a multiple input model with correlated inputs, the sum of ordinary coherences between the inputs and the output may be greater than unity. To address this problem, the ordinary coherence function has been superseded by the cumulative coherence function $\gamma_{M i}^{2}$

$$
\gamma_{M i}^{2}(\omega)=\gamma_{y_{i} x(-1: n)}^{2}(\omega)+\gamma_{z x}^{2}(\omega)=\gamma_{y_{i} x(-1: n)}^{2}(\omega)+\sum_{j=1}^{n} \gamma_{j x(-1 ; j-1)}^{2}(\omega),
$$

where $\gamma_{y_{i} x(-1: n)}^{2}$ is the ordinary coherence function between the $i$ th element of $Y_{(-1: n)}$ and excitation $X$

$$
\gamma_{y_{i} x(-1: n)}^{2}=\frac{\left|S_{y_{i} x(-1: n)}\right|^{2}}{S_{y_{i} y_{i}(-1: n)} S_{x x}} .
$$

It indicates the contribution from the linear spectral component of the response of the $i$ th signal. $\gamma_{j x(-1: j-1)}^{2}$ is the ordinary coherence function between the conditioned spectrum $Z_{j(-1: j-1)}$ and excitation $X$

$$
\gamma_{j x(-1: j-1)}^{2}=\frac{\left|S_{j x(-1: j-1)}\right|^{2}}{S_{j j(-1: j-1)} S_{x x}}
$$

and $\sum_{j=1}^{n} \gamma_{j x(-1: j-1)}^{2}$ indicates the contribution from the nonlinearities.

The cumulative coherence function is always between 0 and 1 and may be considered as a measure of the model accuracy; it is a valuable tool for the selection of an appropriate functional form for the nonlinearity.

\subsubsection{Application example}

The CRP method was applied to the experimental structure depicted in Fig. 1 in (Kerschen et al., 2003a [229]). The identification was carried within the range $0-500 \mathrm{~Hz}$ in which three structural modes exist. For more details about this experiment, the reader is invited to consult (Kerschen et al., 2003a [229]). This structure was also investigated within the framework of the European COST Action F3 (Golinval et al., 2003 [104]).

Figs. 14, 32 and 33 summarise the results obtained. Fig. 14 represents three different FRFs in the vicinity of the first two resonances: (a) the FRF measured using the classical $\mathrm{H}_{2}$ estimate at low level of excitation (i.e., $1.4 \mathrm{Nrms}$ ) for which the geometric nonlinearity is not activated; it should therefore correspond to the FRF of 


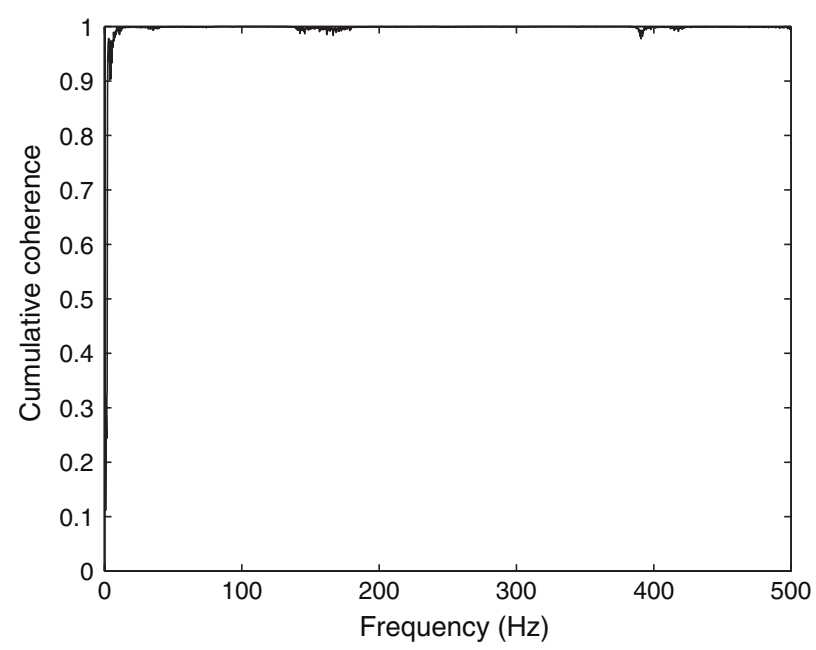

Fig. 32. Cumulative coherence $\gamma_{M 7}^{2}(22 \mathrm{Nrms})$.

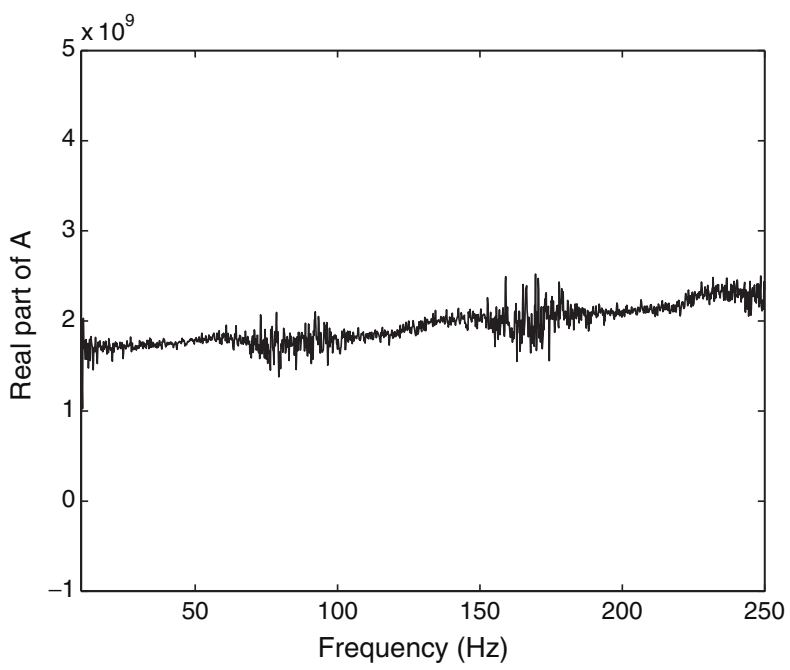

Fig. 33. Real part of the nonlinear coefficient (22 Nrms).

the underlying linear system; (b) the FRF measured using the classical $H_{2}$ estimate at high level of excitation (i.e., $22 \mathrm{Nrms}$ ); (c) the FRF measured using the $H_{c 2}$ estimate at high level of excitation (i.e., $22 \mathrm{Nrms}$ ). It can clearly be observed that the FRF measured using $\mathrm{H}_{2}$ estimate at $22 \mathrm{Nrms}$ is contaminated by the presence of the geometric nonlinearity whereas the FRF measured using $H_{c 2}$ estimate at $22 \mathrm{Nrms}$ is a very accurate estimation of the FRF of the underlying linear system. The accuracy of the identification is confirmed in Fig. 32; overall, the cumulative coherence is close to 1. Fig. 33 represents the real part of the nonlinear coefficient $A$, and its spectral mean performed within the range $10-250 \mathrm{~Hz}$ is equal to $1.96 \times 10^{9}+i 1.55 \times 10^{7} \mathrm{~N} / \mathrm{m}^{2.8}$. As expected, the imaginary part of the coefficient is two orders of magnitude below the real part and can be safely neglected.

A final remark concerns the functional form of the nonlinearity. Although a cubic nonlinearity was expected due to the presence of a geometric nonlinearity, the model $f\left(y_{c}\right)=A\left|y_{c}\right|^{\alpha} \operatorname{sign}\left(y_{c}\right)$ where $y_{c}$ is the response at the bolted connection between the two beams was considered during the identification for greater flexibility. The exponent $\alpha$ was determined by maximising the spectral mean of the cumulative coherence function and was found to be 2.8 . 


\subsubsection{Assessment}

Although it is difficult to draw general conclusions from a single example, it turns out that the CRP method is a very appealing and accurate method for parameter estimation of nonlinear structural models. In addition, the cumulative coherence is a valuable tool for the characterisation of the nonlinearity. The formulation of the method is such that it targets identification of MDOF systems, which enabled the identification of a numerical model with 240 DOFs and two localised nonlinearities (Kerschen and Golinval, 2005a [223]).

An extension of the method to the identification of physical models instead of FRF-based models is discussed in (Kerschen and Golinval, 2005a [223]). In this study, a finite element model of the underlying linear structure is built from the knowledge of the geometrical and mechanical properties of the structure and is updated using linear model updating techniques based upon FRFs (Arruda and Santos, 1993 [422]; Balmes, 1993 [423]; Lin and Ewins, 1994 [424]).

A possible drawback of the method is that it requires the measurements of the structural response at the location of the nonlinearity, which is not always feasible in practice. Also, it is not yet clear how the method would perform in the presence of several nonlinearities, which is typical of a structure with a large number of discrete joints. Finally, future research should investigate how the method could deal with distributed nonlinearities and hysteretic systems modeled using internal state variables (e.g, the Bouc-Wen model).

\subsection{The nonlinear identification through feedback of the output method}

The NIFO formulation (Adams and Allemang, 2000a [232]) is a recent spectral approach for identification of MDOF nonlinear systems. As for the CRP method, the central issue is to eliminate the distortions caused by the presence of nonlinearities in FRFs. It exploits the spatial information and treats the nonlinear forces as internal feedback forces in the underlying linear model of the system.

\subsubsection{Theory}

Let us write the equation of motion in the frequency domain (116) in the form

$$
\mathbf{B}(\omega) \mathbf{Y}(\omega)=\mathbf{X}(\omega)-\sum_{j=1}^{n} \mathbf{A}_{j}(\omega) \mathbf{Z}_{j}(\omega) .
$$

The nonlinear forces may be considered as internal feedback forces and may be evaluated from the measured outputs. For example, in the case of a grounded cubic stiffness at the $i$ th DOF, the nonlinear function vector is

$$
\mathbf{Z}_{1}(\omega)=\mathscr{F}\left\{\left[\begin{array}{llllll}
0 & \ldots & y_{i}(t)^{3} & \ldots & 0
\end{array}\right]^{\mathrm{T}}\right\},
$$

where $\mathscr{F}\{\bullet\}$ is the Fourier transform.

Premultiplying Eq. (131) by the dynamic compliance matrix $\mathbf{H}(\omega)$ yields

$$
\mathbf{Y}(\omega)=\mathbf{H}(\omega) \mathbf{X}(\omega)-\mathbf{H}(\omega) \sum_{j=1}^{n} \mathbf{A}_{j}(\omega) \mathbf{Z}_{j}(\omega)
$$

and,

$$
\mathbf{Y}(\omega)=\left[\begin{array}{llll}
\mathbf{H}(\omega) & \mathbf{H}(\omega) \mathbf{A}_{1}(\omega) & \ldots & \mathbf{H}(\omega) \mathbf{A}_{n}(\omega)
\end{array}\right]\left[\begin{array}{c}
\mathbf{X}(\omega) \\
-\mathbf{Z}_{1}(\omega) \\
\vdots \\
-\mathbf{Z}_{n}(\omega)
\end{array}\right] .
$$

If the external force $\mathbf{X}(\omega)$ and the system response $\mathbf{Y}(\omega)$ are measured, the system described by Eq. (134) may be solved at each frequency; this is carried out in a least-squares system of equations through averaging. An estimation of the FRFs of the underlying linear system $\mathbf{H}(\omega)$ and the nonlinear coefficients $\mathbf{A}_{i}(\omega)$ is therefore available. As for the CRP method, the nonlinear coefficients are frequency dependent, but by performing a spectral mean, the actual values of these coefficients should be retrieved. 
It is noted that Eq. (134) is not considered in its present form. A 'PSD version' of this equation is preferred, which is obtained by using the same procedure as the one used for obtaining Eq. (120) from Eq. (119). The use of PSDs reduces the degree to which linearly correlated terms corrupt the numerical conditioning of the data matrices. An orthogonal least-squares solution (Strang, 1986 [425]) should also be used to reduce the level of ill-conditioning.

\subsubsection{Assessment}

The NIFO technique is similar to the CRP method in several aspects; it is, therefore, an attractive method for nonlinear system identification of MDOF structures. Unlike the CRP method, it is simple to implement, and the estimation of the linear and nonlinear coefficients is carried out in a single step. A possible disadvantage is that the NIFO method does not seem to guarantee the conditioning that is naturally present in the CRP method, as reported in (Kerschen and Golinval, 2002a [426]). The correlation between the linear and nonlinear terms may be an issue, and care must be taken to achieve a good conditioning of the data matrices (e.g., using orthogonal least-squares procedures).

\subsection{The nonlinear resonant decay method}

\subsubsection{Theory}

Classical force appropriation methods (Williams et al., 1986 [256]; Wright et al., 1999 [257]) are used in the identification of linear systems to determine the multi-point force vector that induces single-mode behaviour, thus allowing each normal mode to be identified in isolation. For a proportionally damped linear structure, the final model consists of a set of uncoupled SDOF oscillators in modal space.

An extension of the force appropriation approach to the identification of non-proportionally damped linear systems, termed the resonant decay (RD) method, is presented in (Naylor et al., 2004 [427]). An appropriated force pattern with a single sine wave is applied as a 'burst' to excite a given mode of interest. Once the excitation ceases, the free decay of the system includes a response from any modes coupled by damping forces to the mode being excited. A curve fit to a limited subset of modes can then be performed to yield any significant damping terms which couple the corresponding SDOF oscillators.

A generalisation of this methodology for identification of nonlinear systems is described in this section. For the analysis of large nonlinear structures with high modal density in a broad frequency range, an enormous number of parameters is to be identified because the nonlinear modal restoring forces $\mathbf{f}_{m}(\mathbf{u}, \dot{\mathbf{u}})$ are potentially functions of the many modal displacements $u_{i}(t)$ and/or velocities $\dot{u}_{i}(t)$ (in other words, the nonlinearity may be responsible for many terms coupling the SDOF oscillators); this renders parameter estimation intractable.

The method developed in (Wright et al., 2001 [255]) offers a practical solution to this critical issue by proposing a multi-stage identification of the linear modal space-based model in which the initial estimation problem is replaced by a sequence of low-dimensional problems. At this point, we note that the selective sensitivity approach developed in (Ben-Haim, 1993 [428]) also proposes to identify the entire system via a sequence of low-dimensional estimation problems through the use of selective excitation. In (Wright et al., 2001 [255]), the scale of the identification problem is reduced by classifying the modes ${ }^{5}$ into different categories: (i) linear proportionally damped modes, well separated in frequency; (ii) linear proportionally damped modes, very close in frequency; (iii) linear non-proportionally damped modes; (iv) modes influenced by nonlinear effects with no significant nonlinear coupling to other modes; and (v) modes influenced by nonlinear effects with significant nonlinear coupling to other modes. The set of uncoupled SDOF oscillators in modal space is therefore enhanced by the inclusion of modal damping cross-coupling terms for nonproportionally damped modes, 'direct' nonlinear terms $f_{m}\left(u_{j}, \dot{u}_{j}\right)$ if the $j$ th mode behaves nonlinearly and nonlinear cross-coupling terms $f_{m}\left(u_{i}, \dot{u}_{i}, u_{j}, \dot{u}_{j}\right)$ if the $i$ th and $j$ th modes are nonlinearly coupled.

Modes of type (i) may be identified using classical curve-fitting methods. Modes of type (ii) may benefit from identification using force appropriation. Force appropriation and the RD method are suitable for modes of type (iii). Anticipating that only a relatively small portion of modes will actually behave in a nonlinear fashion for most structures (this assumption implies that the method targets weakly nonlinear systems), two

\footnotetext{
${ }^{5}$ It is emphasised that a mode refers to the mode of the underlying linear system; the discussion does not refer to the NNMs.
} 
methodologies which enable the treatment of modes affected by nonlinearity (i.e., modes of type (iv) and (v)) individually or in small groups were developed:

- The FANS method (Atkins et al., 2000 [258]) extends the classical linear force appropriation approach to nonlinear systems through the use of a force pattern that includes higher harmonic terms. The parameters are optimised such that the nonlinear coupling terms are counteracted, which prevents any response other than the mode of interest. The direct linear and nonlinear terms for that mode may be estimated using a classical SDOF RFS identification.

- The NLRD method (Wright et al., 2001 [255]) is an extension of the RD method to nonlinear systems and enables small groups of modes to be excited. A classical appropriated force pattern with a single sine wave is applied as a 'burst' to excite a given mode of interest 'approximately'. If the mode is uncoupled nonlinearly, then it should dominate the response in the steady state phase. If it is nonlinearly coupled, other modes may also exhibit a significant response. During the decay, the presence of linear damping couplings as well as nonlinear couplings between the modes is apparent. A 'low-order' regression analysis in modal space using the RFS method is then carried out for identification of direct and cross-coupling terms.

The NLRD method is applied in (Wright et al., 2001 [255]) to a 5-DOF spring-mass system clamped at both extremities and designed to be symmetric in its linear components. The system has a cubic stiffness nonlinearity between the second and fourth DOFs. The system is linear in modes 1, 3 and 5; modes 2 and 4 are nonlinear and coupled together. In order to illustrate the burst principle, a burst is applied to excite mode 5 as shown in Fig. 34. ${ }^{6}$ Because mode 5 behaves linearly and the correct appropriated force vector is used, no modal force is input to the other modes, and only mode 5 responds. Consider now a burst applied to mode 4 as shown in Fig. 35. There is only a modal force for mode 4 but now mode 2 responds due to the nonlinear coupling. Modes 1, 3 and 5 are not excited because of the force appropriation. A curve fitting can then be carried out for mode 4 using only the modal responses associated with modes 2 and 4; the scale of the identification has been effectively reduced.

\subsubsection{Assessment}

Although this nonlinear system identification technique has not yet been applied to large continuous structures, the authors believe that it paves the way for the analysis of practical systems with high modal density. Because modes are treated individually or in small groups, the method has the inherent ability to 'split' the original and complex identification problem into a sequence of much simpler and smaller problems. One may also account for non-proportional damping, which is another interesting feature of the method.

Imperfection force appropriation and modal matrix may reduce the accuracy of the identification as discussed in (Wright et al., 2001 [255]). As a result, the number, location and pattern of excitation sources should be determined in a judicious manner in order for this process to be successful; shaker-structure interaction may also be an issue for light-weight structures.

\subsection{Structural model updating}

\subsubsection{Theory}

The structural model updating process is presented in diagram form in Fig. 36. It can be decomposed into four steps: (1) experimental measurements and structural modeling; (2) feature extraction and correlation study; (3) selection of the updating parameters and (4) minimisation of the objective function. The success of model updating is conditional upon each step being properly carried out.

It is noted that the emphasis in the present section is put upon model updating using time-domain measurements.

Experimental measurements and structural modeling: Experiment design (e.g, selection of excitation sources, number and location of sensors) is a crucial step but, as stated in Section 1 of this paper, it is not discussed in

\footnotetext{
${ }^{6}$ The results in Figs. 34 and 35 were obtained by Dr. Jan Wright and co-workers - the authors are very grateful for permission to use them.
} 

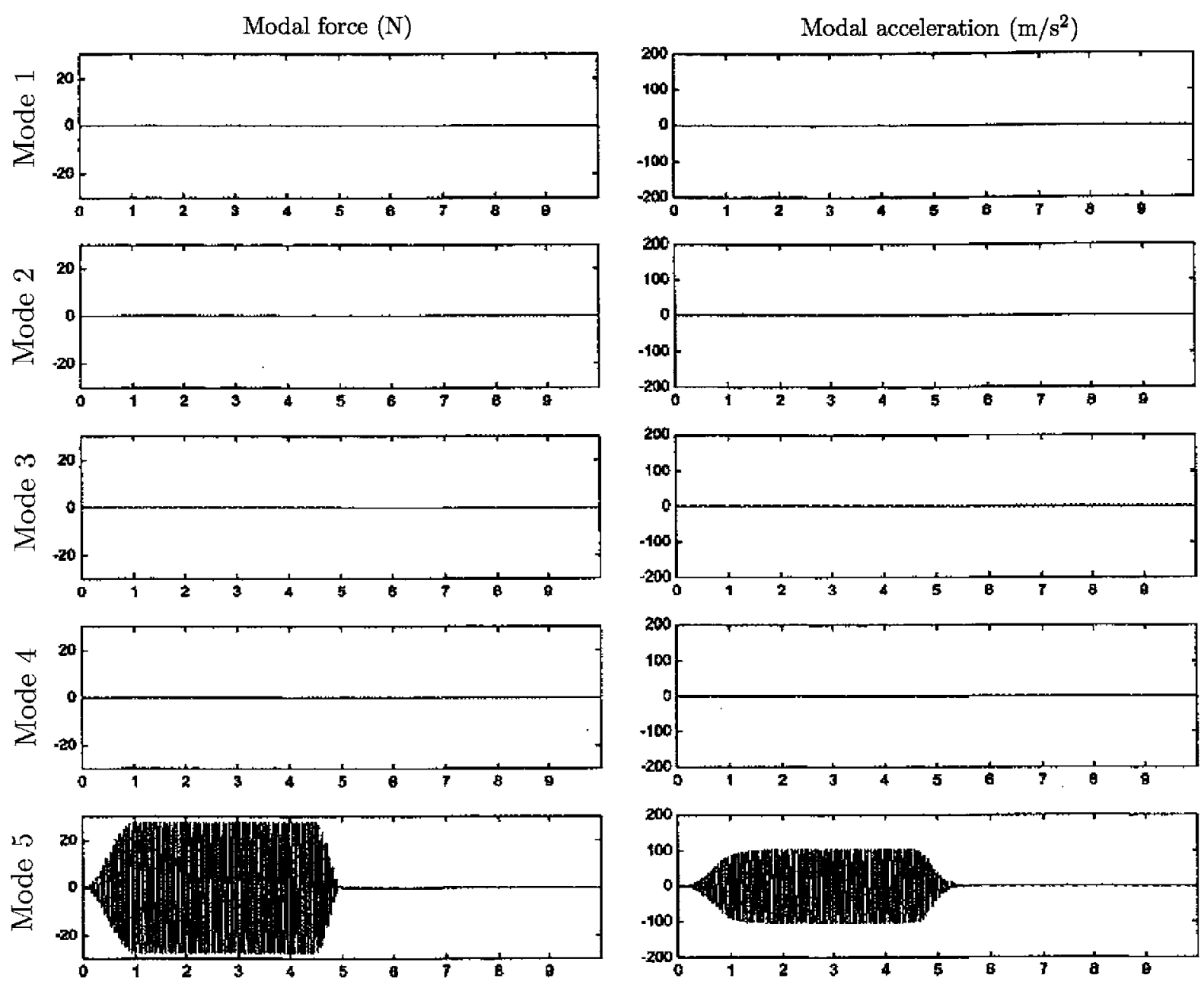

Time (s)

Fig. 34. Modal forces and responses to burst excitation of mode 5 using perfect appropriation (Wright et al., 2001 [255]).

this overview. It is therefore assumed that vibration tests have been performed on the real structure; a matrix $\mathbf{Y}(t)$ containing $m$ samples of the response (e.g., acceleration data) measured at $n$ different locations on the structure is formed

$$
\mathbf{Y}(t)=\left[\begin{array}{lll}
\mathbf{y}\left(t_{1}\right) & \cdots & \mathbf{y}\left(t_{m}\right)
\end{array}\right]=\left[\begin{array}{ccc}
y_{1}\left(t_{1}\right) & \cdots & y_{1}\left(t_{m}\right) \\
\cdots & \cdots & \cdots \\
y_{n}\left(t_{1}\right) & \cdots & y_{n}\left(t_{m}\right)
\end{array}\right] .
$$

From the knowledge of the geometrical and mechanical properties of the structure, a structural model can be created. By imposing in this model the same excitation conditions $\mathbf{x}(t)$ as for the real structure, the structural response can be predicted using time-integration algorithms; the matrix $\hat{\mathbf{Y}}(t)$ is obtained. At this stage, verification, i.e., 'solving the equations correctly' (Roache, 1998 [59]), is necessary, but its description would take us too far afield.

Feature extraction and correlation study: Matrix $\hat{\mathbf{Y}}(t)$ generally differs from $\mathbf{Y}(t)$ due to three sources of errors, namely modeling errors (e.g, imperfect boundary conditions or assumption of proportional damping), parameter errors (e.g., inaccuracy of Young's modulus) and testing errors (e.g, noise during the measurement process). However, estimating the predictive capability of a structural model based only on its ability to match 

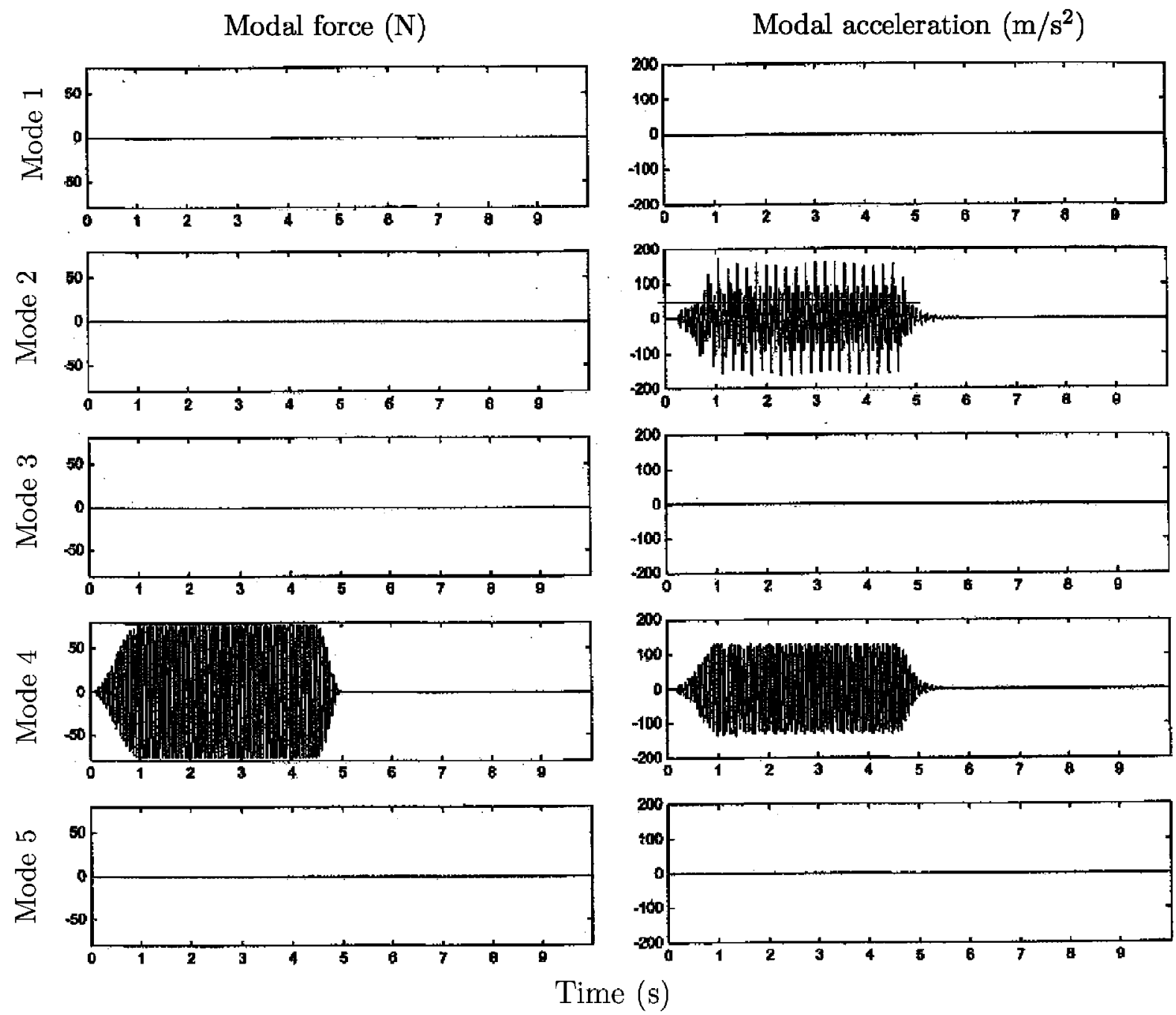

Fig. 35. Modal forces and responses to burst excitation of mode 4 using perfect appropriation ((Wright et al., 2001 [255]).

measured time series may be hazardous. The comparison between experimental features $\mathbf{f}_{i}$ and predicted features $\hat{\mathbf{f}}_{i}$ should be preferred. In linear dynamics, natural frequencies and mode shapes provide a sound basis for ascertaining whether the prediction of the model will adequately represent the overall dynamic response of the structure. Another well-established technique is to use data in the frequency domain because the effort of experimental modal analysis is avoided, and averaging to reduce noise effects is straightforward.

When performing test-analysis correlation for nonlinear structures, the features commonly defined for linear structures do no longer provide an accurate characterisation of the dynamics, as explained in the tutorial section. The definition of features that enhance the effect of nonlinearity on the structural behaviour is therefore necessary. NNMs provide a valuable theoretical tool for understanding dynamic phenomena such as mode bifurcations and nonlinear mode localisation but it is a little early to tell if they will be of substantial help for structural model updating. For this reason, other features have been considered in the technical literature as discussed in Section 3.7.

Selection of the updating parameters: If correlation is not satisfactory, the structural model is to be updated. The correction of the model begins with the selection of the updating parameters. Parameter selection is a difficult and critical step, and the success of the model updating process is conditional upon the ability to 


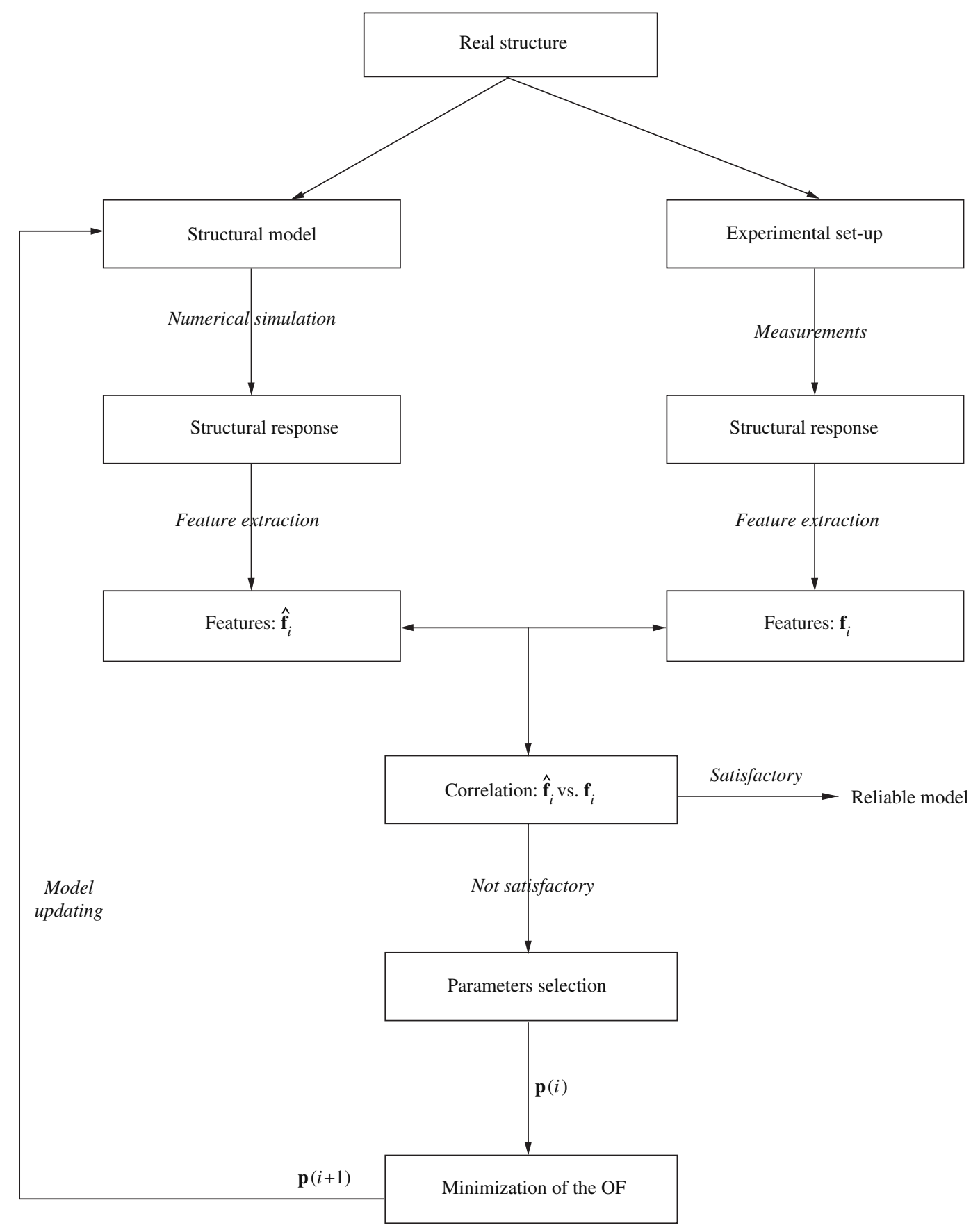

Fig. 36. Model updating sequence of non-linear systems (OF: objective function).

identify the adequate parameters. For this purpose, error localisation techniques and sensitivity analysis may be useful (Friswell and Mottershead, 1995 [312]; Link, 1999 [313]), but physical understanding of the structural behaviour and engineering judgment play the key role (see for instance (Mares et al., 2003 [429])).

Minimisation of the objective function: New values of the updating parameters are computed through the minimisation of an objective function $J$

$$
\min _{\mathbf{p}} J=\|R(\mathbf{p})\|^{2},
$$


where vector $\mathbf{p}$ contains the updating parameters. The residue $R(\mathbf{p})$ may simply be the norm of the difference between the predicted and experimental features. The objective function is generally nonlinear with respect to the updating parameters, and it is necessary to use optimisation algorithms to perform the minimisation.

\subsubsection{Application example}

Structural model updating was applied to the experimental system depicted in Fig. 1 in (Lenaerts et al., 2003 [264]). This structure was also investigated within the framework of the European COST Action F3 (Golinval et al., 2003 [104]). An impulsive force was imparted to the cantilever beam using an impact hammer, and the structural response was measured using seven accelerometers evenly spaced across the beam.

A structural model was created using the finite element method, and the effect of the geometric nonlinearity was modeled with a grounded spring at the connection between the cantilever beam and the short beam. The accelerations of the numerical model were computed using Newmark's method.

The correlation study was performed by comparing experimental and predicted POMs. Although the POMs do not have the theoretical foundations of the NNMs, they do provide a good characterisation of the dynamics of a nonlinear system. Another advantage is that their computation is straightforward; it involves a singular value decomposition of the response matrix $\mathbf{Y}(t)$

$$
\mathbf{Y}=\mathbf{U} \boldsymbol{\Sigma} \mathbf{V}^{\mathrm{T}},
$$

where each column of matrix $\mathbf{U}$ contains a POM. Matrix $\boldsymbol{\Sigma}$ gives information about the participation of the POMs in the system response whereas their amplitude modulations are contained in matrix $\mathbf{V}$. Insight into the frequency of oscillation of the POMs is available by applying the wavelet transform to matrix V. For a
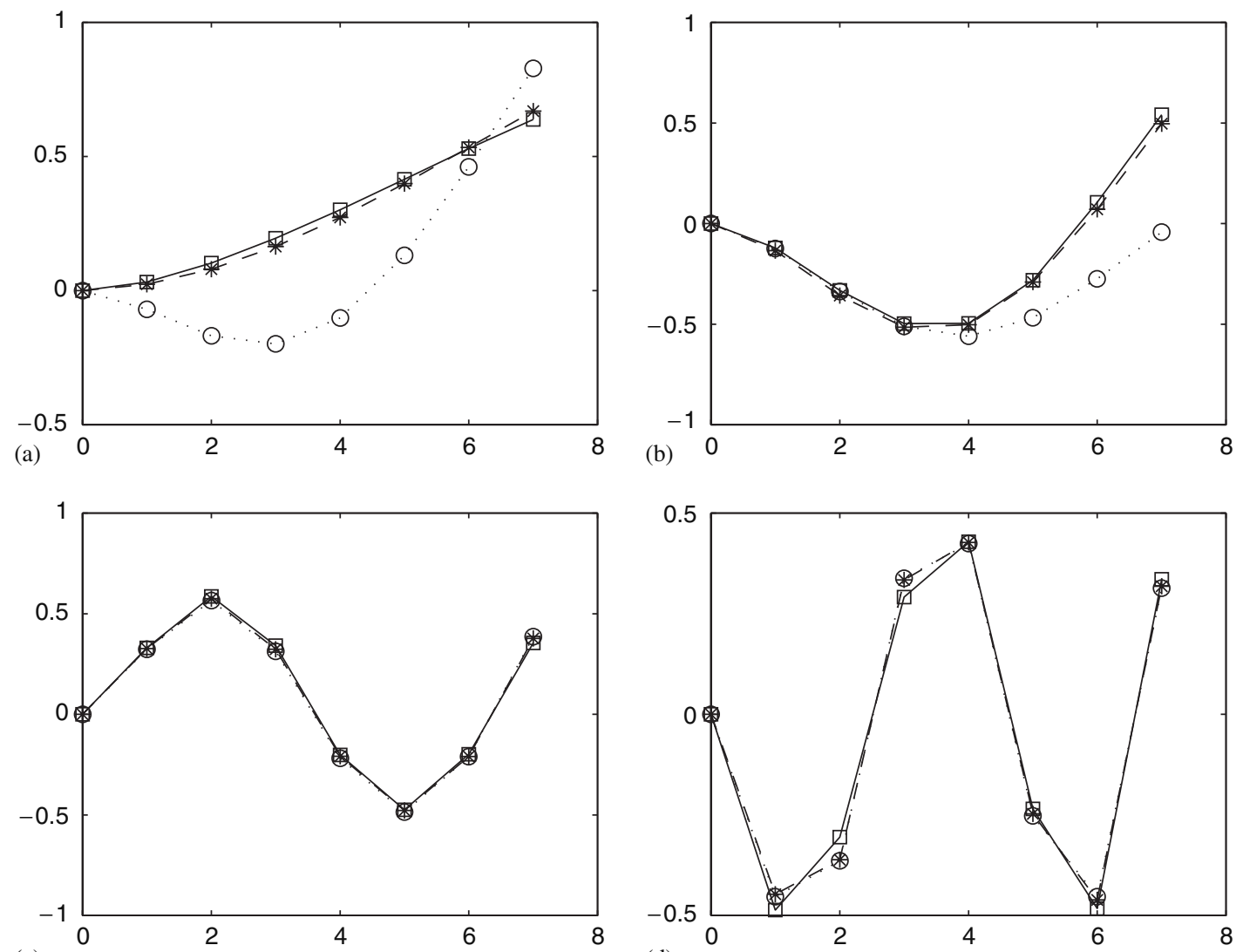

(c)

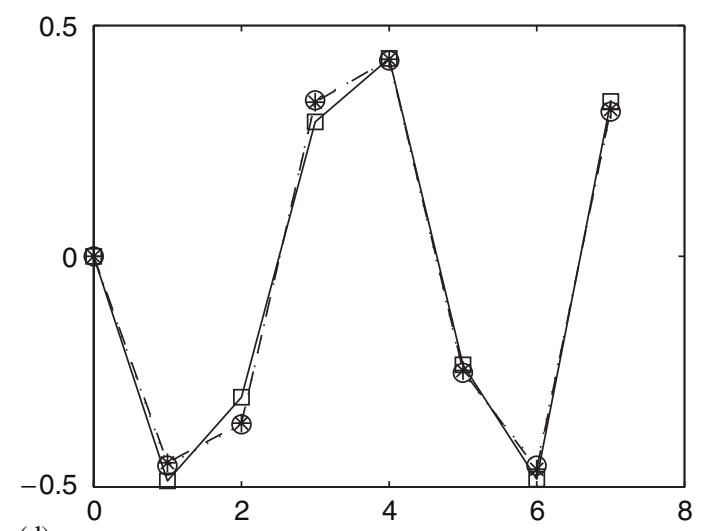

(d)

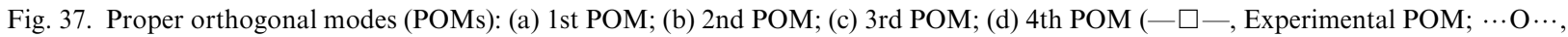
initial finite element model; —*-, updated finite element model). 


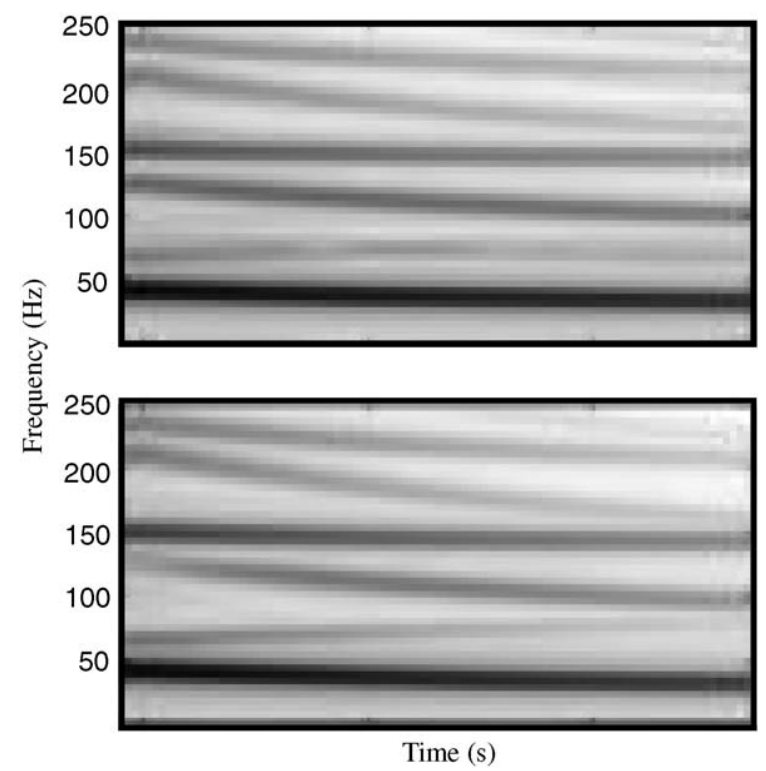

Fig. 38. Wavelet transform of the amplitude modulation of the first POM. Top plot: experimental structure; bottom plot: updated finite element model.

detailed description of the POD, the reader is invited to consult (Holmes et al., 1996 [430]), and an overview of the POD for dynamical characterisation of nonlinear structures is available in (Kerschen et al., 2005c [431]). Fig. 37 shows that the first two POMs predicted by the initial finite element model are not in close agreement with those of the experimental structure. Because these two POMs account for more than $90 \%$ of the total energy contained in the system response, the model must be improved.

Several parameters were not known precisely in the initial model, especially the stiffness of the bolted connection between the two beams and the coefficient and exponent of the nonlinearity; they were thus selected as updating parameters. After optimisation, the coefficient and exponent of the nonlinearity were $1.65 \times 10^{9} \mathrm{~N} / \mathrm{m}^{2.8}$ and 2.8 , respectively, which is in good concordance with the estimates given by the CRP method (see Section 7.1.2). There is now a satisfactory match between the experimental POMs and those predicted by the updated finite element model as shown in Fig. 37. Fig. 38 displays the wavelet transform of the amplitude modulation of the first POM; the dominant frequency component is around $50 \mathrm{~Hz}$, but harmonics - a typical feature of nonlinear systems - can also clearly be observed. There is also a good agreement between the experimental and numerical results in Fig. 38, which confirms that the updated model has a good predictive accuracy.

\subsubsection{Assessment}

Structural model updating has the inherent ability to provide reliable models of more complex nonlinear structures. For instance, numerical examples with a few hundred DOFs are investigated in (Hasselman et al., 1998 [262]; Meyer and Link, 2002 [319]; Kerschen, 2003c [324]; Kerschen and Golinval, 2004a [325]), whereas a fully integrated experimental system is considered in (Hemez and Doebling, 2001a [103]).

However, several crucial issues remain largely unresolved, and there is much research to be done:

- There are no universal features applicable to all types of nonlinearities; test-analysis correlation is still a difficult process.

- It is generally assumed that the analyst has the ability to formulate an appropriate initial model and to identify precisely the source and location of the erroneous parameters; these are extremely challenging tasks when dealing with complex structures.

- Many of the error criteria formulations lead to objective functions with a highly nonlinear solution space; multiple parameter sets may potentially yield equally good reproduction of the experimental measurements, 
especially when limited measurement data is available (We note that info-gap models may offer an elegant solution to this problem (Hemez and Ben-Haim, 2004 [432])). In addition, the initial model cannot be assumed to be close to the 'actual' model because a priori knowledge about nonlinearity is often limited; the starting point of the optimisation may be far away from the sought minimum. For all these reasons, objective function minimisation may be challenging and time consuming.

\section{Summary and future research needs}

This survey paper reviews the past and recent developments in nonlinear system identification, the objective of which is to produce high fidelity models that may be used for purposes such as

- virtual prototyping; this encompasses the selection of optimal system parameters in order to meet specific design goals, the prediction of the occurrence of undesirable instabilities and bifurcations (e.g., aeroelastic instabilities), the impact of structural modifications and the study of the effects of structural, environmental or other types of uncertainties on the robustness of operation;

- development of diagnostic and prognostic tools that enable simple, accurate, economic, and preferably online detection of structural faults at an early stage of their developments before they become catastrophic for the operation of the system;

- structural control, e.g, the control of mechatronic systems or of structural vibrations produced by earthquake or wind.

There is a substantial body of literature on nonlinear system identification; it should be recalled that the paper is inevitably biased toward those areas the authors are most familiar with. Some of the popular methods in this research area were discussed, and numerical and experimental examples were presented to illustrate their fundamental concepts but also their assets and limitations. The main differences between linear and nonlinear oscillations were also detailed in a tutorial.

Because of the highly individualistic nature of nonlinear systems and because the basic principles that apply to linear systems and that form the basis of modal analysis are no longer valid in the presence of nonlinearity, one is forced to admit that there is no general analysis method that can be applied to all systems in all instances. As a result, numerous methods for nonlinear system identification have been developed during the last three decades. A large proportion of these methods were targeted to SDOF systems, but significant progress in the identification of MDOF lumped parameter systems has been realised recently. To date, simple continuous structures with localised nonlinearity are within reach.

For simple structures or approximate models of more complex structures, it is reasonable to estimate all the model parameters. However, for the analysis of structures with a large number of DOFs and with a high modal density in a broad frequency range, resorting to multi-parameter complex structural models is inevitable. This critical issue begins to be resolved by several recent approaches among which we can cite:

- Frequency-domain methods such as the CRP and NIFO methods have, in principle, the capability of identifying the dynamics of large structures. In addition to the nonlinear coefficients, they compute a FRFbased model of the underlying linear structure directly from the experimental data, which facilitates the identification process.

- The NLRD method proposes to classify the modes into different categories (i.e., influenced or not by nonlinear effects, coupled or uncoupled in damping and/or nonlinearity), which enables the treatment of modes individually or in small groups. This technique does not decrease the number of parameters to be estimated, but it simplifies the parameter estimation process by targeting a multi-stage identification.

- Structural model updating techniques exploit the knowledge of the geometric and mechanical properties to determine an initial model of the structure, many parameters of which are usually accurately computed and do not have to be identified from experimental data.

All these methods have their own drawbacks, but they show promise in the challenging area of nonlinear system identification. 
Besides rendering parameter estimation tractable, other important issues must be addressed adequately to progress toward the development of accurate, robust, reliable and predictive models of large, threedimensional structures with multiple components and strong nonlinearities. The following discussion presents some of the key aspects that, we believe, will drive the development of nonlinear system identification in the years to come.

(i) We cannot stress enough the importance of having an accurate characterisation of the nonlinear elastic and dissipative behaviour of the physical structure prior to parameter estimation. Without a precise understanding of the nonlinear mechanisms involved, the identification process is bound to failure. Characterisation is a very challenging step because nonlinearity may be caused by many different mechanisms and may result in plethora of dynamic phenomena. Some 'real-life' nonlinear effects only begin to be adequately modeled (e.g., the dynamics of structures with bolted joints (Gaul and Lenz, 1997 [376]; Hartwigsen et al., 2004 [377], Song et al., 2004 [301])); some are still far from being understood (e.g, experiments reported in (Quinby and Feeny, 2004 [433]) showed that quasi-periodic responses in a frictionally excited beam may involve very low frequencies at subharmonic orders of 20 to 130). The lack of knowledge about nonlinearity is sometimes circumvented by nonlinear black-box models such as those proposed in (Kosmatopoulos et al., 2001 [298]; Fan and Li, 2002 [303]; Peifer et al., 2003 [305]), but, in our opinion, a priori information and physics-based models should not be superseded by any 'blind' methodology. Careful and systematic studies of nonlinear dynamical effects such as those carried out in (Richards and Singh, 2001 [19]; Malatkar and Nayfeh, 2003c [354]; Caffrey et al., 2004 [434]) are strongly encouraged and are a necessary step toward the development of accurate nonlinear structural models. Improving our knowledge and our modeling capabilities of the range of possible nonlinear behaviours (this also reduces the level of uncertainty and increases our confidence in the model; see (iii)) is therefore a crucial need, especially because structural dynamics is becoming increasingly nonlinear, addressing multi-physics phenomena (Hammond, 2001 [435]; Hemez and Doebling, 2001a [103]).

(ii) Most of the analytical techniques currently available are limited to the steady-state response of weakly nonlinear oscillators. On the other hand, because strong nonlinearity is more and more encountered in practical applications, new dynamical phenomena are observed that have to be accounted for. For example, it is only recently that resonance capture phenomena which are mainly of a transient nature have been reported in the structural dynamics literature (Quinn et al., 1995 [436]; Quinn, 1997 [95]; Vakakis and Gendelman, 2001 [51]). As a result, there is the need for new analytical developments enabling the study of the transient dynamics of strongly nonlinear oscillators. Such developments will provide better insight into the dynamics of interest, thereby facilitating the characterisation of the nonlinear behaviour discussed in (i).

(iii) The concept of NNM offers a solid theoretical and mathematical framework for analysing and interpreting a wide class (but not the entirety!) of nonlinear dynamical phenomena, and yet it has a clear and simple conceptual relation to the classical linear normal mode, with which practicing vibration engineers are familiar. Viewed in this context, the concept of NNM can provide the appropriate framework for closer collaboration and mutual understanding between Academia and Industry. To formulate practical NNM-based nonlinear system identification techniques, advances in a number of critical research areas need to be accomplished including

- the development of efficient computational algorithms for studying the NNMs of practical (multiDOF, flexible or large-scale) mechanical systems and their bifurcations;

- the study of possible exact or approximate (for example, asymptotic) NNM-based superposition principles for expressing nonlinear responses as nonlinear superpositions of component responses;

- the study of possible exact or approximate (energy dependent) orthogonality relations satisfied by NNMs that would permit their use as bases for order reduction of the nonlinear dynamics; we mention at this point the computational studies of S. Shaw, C. Pierre and co-workers (Pesheck et al., 2001a,b, 2002a,b [254,437-439] and Apiwattanalunggarn et al., 2003 [440]) show that (ad hoc) NNM-based Galerkin expansions lead to more accurate numerical computations of the responses of flexible systems, compared to linear eigenfunction-based expansions; 
- the examination of the relation of NNMs to computational bases extracted by techniques such as wavelet analysis and linear or nonlinear POD (some preliminary results on relation between NNMs and POMs, and between NNMs and nonlinear POMs are reported in (Feeny and Kappagantu, 1998 [441]; Feeny, 2002 [442]; Kerschen and Golinval, 2002b [443]) and (Kerschen and Golinval, 2004b [444]), respectively);

- the examination of the relation between NNMs and Volterra series expansions/HOFRFs; also, of the relation of NNMs to already studied nonlinear superposition techniques for special classes of dynamical systems.

(iv) All systems referenced in this paper are assumed to be deterministic. Because there will always be some degree of uncertainty in the numerical models due to unknown physics, environmental variability, economics of modeling for parameter estimation, uncertain inputs, manufacturing tolerances, assembly procedures, idealisation errors, etc., the issues of uncertainty quantification and propagation, and of numerical predictability are central questions when it comes to assessing whether a simulation is capable of reproducing with acceptable accuracy the experiment it is supposed to replace. To this end, fundamental questions such as the following need to be addressed (Hemez and Doebling, 2000 [102]):

1. Are the experiments and simulations consistent statistically speaking?

2. What is the degree of confidence associated with the first answer?

3. If additional data sets are available, by how much does the confidence increase?

Such questions are progressively being addressed in the structural dynamics community by considering nonlinear system identification as an integral part of the V\&V process (Hemez et al., 2005 [64]).

(v) Research should focus more on testing of practical structures in their own operating environment, rather than on laboratory tests of representative structures. Algorithms for optimally deploying sensors and exciters along the structure are not yet fully developed. The ability to use vibrations induced by ambient environmental or operating loads is an area that merits further investigation; this will demand to reduce the dependence upon measurable excitation forces, as attempted in (Yuen and Beck, 2003 [320]; Haroon et al., 2005 [160]). On-line identification is also important for applications such as structural health monitoring (Smyth et al., 2002 [445]; Yang and Lin, 2004a [446]).

To conclude this paper, it is fair to say that, even if one cannot foresee the arrival of a paradigm shift, it can be safely predicted that during the next 10 years a 'universal' technique capable of addressing nonlinear dynamical phenomena of every possible type in every possible structural configuration will not be developed. It is therefore likely that nonlinear system identification will have to retain its current 'toolbox' philosophy, with (hopefully) more powerful methodologies, techniques and algorithms of increased sophistication being added. In the future, the stage will be (hopefully) reached, where attempts to unify and combine the most powerful and reliable methods will be initiated.

\section{Acknowledgements}

One of the authors (GK) is supported by a grant from the Belgian National Fund for Scientific Research (FNRS) which is gratefully acknowledged.

\section{Appendix. List of acronyms}
ARMA
ARX
BVP
COST
CRP
DOF
DPE

auto-regressive moving average

auto-regressive with exogeneous inputs

boundary value problem

cooperation in the field of scientific and technical research

conditioned reverse path

degree of freedom

direct parameter estimation 


ECL
FANS
FFT
FRF
FT
HOFRF
IR
IMF
mdof
NARMAX
NARX
NIFO
NLRD
NMSE
NNM
POD
POM
PSD
RC
RD
RFS
RP
SDOF
SRC
TDNN
TRC
V \& V

ecole centrale de Lyon

force appropriation of nonlinear systems

fast Fourier transform

frequency response function

Fourier transform

higher-order frequency response function

internal resonance

intrinsic mode function

multi-degree-of-freedom

nonlinear auto-regressive moving average with exogeneous inputs nonlinear auto-regressive with exogeneous inputs

nonlinear identification through feedback of the outputs

nonlinear resonant decay

normalised mean square error

nonlinear normal mode

proper orthogonal decomposition

proper orthogonal mode

power spectral density

resonance capture

resonant decay

restoring force surface

rational polynomial

single-degree-of-freedom

sustained resonance capture

time-delay neural network

transient resonance capture

verification and validation

\section{References}

[1] E.A. Bender, An Introduction to Mathematical Modeling, Dover, New York, 2000 (Section 1).

[2] H. Van Der Auweraer, Testing in the age of virtual prototyping, in: Proceedings of International Conference on Structural Dynamics Modelling, Funchal, 2002 (Section 1).

[3] L. Ljung, System Identification-Theory for the User, Prentice-Hall, Englewood Cliffs, 1987 (Section 1).

[4] T. Soderstrom, P. Stoica, System Identification, Prentice-Hall, Englewood Cliffs, 1989 (Section 1).

[5] S.R. Ibrahim, E.C. Mikulcik, A time domain modal vibration test technique, Shock and Vibration Bulletin 43 (1973) 21-37 (Section 1).

[6] J.S. Juang, R.S. Pappa, An eigensystem realization algorithm for modal parameter identification and model reduction, AIAA Journal of Guidance, Control and Dynamics 12 (1985) 620-627 (Section 1).

[7] P. Van Overschee, B. De Moor, Subspace Identification for Linear Systems: Theory, Implementation, Applications, Kluwer Academic Publishers, Dordrecht, 1996 (Section 1).

[8] B. Peeters, H. Van Der Auweraer, P. Guillaume, The PolyMAX frequency domain method: a new standard for modal parameter estimation, Shock and Vibration 11 (2004) 395-409 (Section 1).

[9] W. Heylen, S. Lammens, P. Sas, Modal Analysis Theory and Testing, KUL Press, Leuven, 1997 (Sections 1, 3.4).

[10] N.M.M. Maia, J.M.M. Silva, Theoretical and Experimental Modal Analysis, Research Studies Press LTD, Taunton, 1997 (Sections 1, 3.4).

[11] D.J. Ewins, Modal Testing: Theory, Practice and Application, second ed., Research Studies Press LTD, Hertfordshire, 2000 (Sections 1, 3.4, 4.1, 6.5, 6.6; Introduction Section 3).

[12] R.J. Allemang, D.L. Brown, A unified matrix polynomial approach to modal identification, Journal of Sound and Vibration 211 (1998) 301-322 (Section 1).

[13] R.J. Allemang, A.W. Phillips, The unified matrix polynomial approach to understanding modal parameter estimation: an update, in: Proceedings of the International Seminar on Modal Analysis (ISMA), Leuven, 2004 (Section 1).

[14] M. Amabili, M. Paidoussis, Review of studies on geometrically nonlinear vibrations and dynamics of circular cylindrical shells and panels, with and without fluid-structure interaction, Applied Mechanics Reviews 56 (2003) 349-381 (Section 1).

[15] A.H. Nayfeh, P.F. Pai, Linear and Nonlinear Structural Mechanics, Wiley-Interscience, New York, 2004 (Section 1). 
[16] S.W. White, S.K. Kim, A.K. Bajaj, P. Davies, D.K. Showers, P.E. Liedtke, Experimental techniques and identification of nonlinear and viscoelastic properties of flexible polyurethane foam, Nonlinear Dynamics 22 (2000) 281-313 (Sections 1, 5.1).

[17] J.F. Schultze, F.M. Hemez, S.W. Doebling, H. Sohn, Application of non-linear system model updating using feature extraction and parameter effect analysis, Shock and Vibration 8 (2001) 325-337 (Sections 1, 3.7, 5.1).

[18] R. Singh, P. Davies, A.K. Bajaj, Identification of nonlinear and viscoelastic properties of flexible polyurethane foam, Nonlinear Dynamics 34 (2003) 319-346 (Sections 1, 5.1).

[19] C.M. Richards, R. Singh, Characterization of rubber isolator nonlinearities in the context of single- and multi-degree-of-freedom experimental systems, Journal of Sound and Vibration 247 (2001) 807-834 (Sections 1, 5.1, 8).

[20] T.K. Caughey, A. Vijayaraghavan, Free and forced oscillations of a dynamic system with linear hysteretic damping, International Journal of Non-Linear Mechanics 5 (1970) 533-555 (Section 1).

[21] G.R. Tomlinson, J.H. Hibbert, Identification of the dynamic characteristics of a structure with Coulomb friction, Journal of Sound and Vibration 64 (1979) 233-242 (Section 1).

[22] H.A. Sherif, T.M. Abu Omar, Mechanism of energy dissipation in mechanical system with dry friction, Tribology International 37 (2004) 235-244 (Section 1).

[23] F. Al-Bender, W. Symens, J. Swevers, H. Van Brussel, Theoretical analysis of the dynamic behavior of hysteresis elements in mechanical systems, International Journal of Non-Linear Mechanics 39 (2004) 1721-1735 (Section 1).

[24] M. Juntunen, J. Linjama, Presentation of the VTT benchmark, Mechanical Systems and Signal Processing 17 (2003) 179-182 (Sections 1; Introduction Section 3).

[25] V.I. Babitsky, V.L. Krupenin, Vibrations of Strongly Nonlinear Discontinuous Systems, Springer, Berlin, 2001 (Sections 1, 2.1).

[26] S.H. Rhee, P.H.S. Tsang, S.W. Yen, Friction-induced noise and vibrations of disc brakes, Wear 133 (1989) 39-45 (Section 1).

[27] T. Von Karman, The engineer grapples with nonlinear problems, Bulletin of the American Mathematical Society 46 (1940) 615-683 (Section 1).

[28] A.H. Nayfeh, D.T. Mook, Nonlinear Oscillations, Wiley-Interscience, New York, 1979 (Sections 1, 2.1, 3.1, 5.1, 6.5; Introduction Section 3).

[29] S.H. Strogatz, Nonlinear Dynamics and Chaos: With Applications to Physics, Biology, Chemistry, and Engineering, AddisonWesley, Reading, MA, 1994 (Sections 1; Introduction Section 3).

[30] F. Verhulst, Nonlinear Differential Equations and Dynamical Systems, second ed., Springer, Berlin, 1999 (Sections 1; Introduction Section 3).

[31] R. Rand, Lecture Notes on Nonlinear Vibrations, Cornell, 2003. Notes freely available at http://tam.cornell.edu/rand.html. (Section 1; Introduction Section 3)

[32] J. Guckenheimer, P. Holmes, Nonlinear Oscillations, Dynamical Systems and Bifurcation of Vector Fields, Springer, New York, 1983 (Sections 1, 2.1; Introduction Section 3).

[33] S. Wiggins, Introduction to Applied Nonlinear Dynamical Systems and Chaos, Springer, New York, 1990 (Sections 1, 2.1; Introduction Section 3).

[34] T.K. Caughey, Equivalent linearisation techniques, Journal of the Acoustical Society of America 35 (1963) 1706-1711 (Sections 1, 3.1).

[35] W.D. Iwan, A generalization of the concept of equivalent linearization, International Journal of Non-Linear Mechanics 8 (1973) 279-287 (Sections 1, 3.1).

[36] R.M. Rosenberg, The normal modes of nonlinear $n$-degree-of-freedom systems, Journal of Applied Mechanics 29 (1962) 7-14 (Sections 1, 3.4).

[37] R.M. Rosenberg, On nonlinear vibrations of systems with many degrees of freedom, Advances in Applied Mechanics 9 (1966) 155-242 (Sections 1, 2.1, 3.4).

[38] R. Rand, A direct method for nonlinear normal modes, International Journal of Non-Linear Mechanics 9 (1974) 363-368 (Sections 1, 3.4).

[39] S.W. Shaw, C. Pierre, Normal modes for non-linear vibratory systems, Journal of Sound and Vibration 164 (1993) 85-124 (Sections 1, 2.1, 3.4).

[40] A.F. Vakakis, L.I. Manevitch, Y.V. Mikhlin, V.N. Pilipchuk, A.A. Zevin, Normal Modes and Localization in Nonlinear Systems, Wiley, New York, 1996 (Sections 1, 2.1, 3.4).

[41] A.F. Vakakis, Non-linear normal modes and their applications in vibration theory: an overview, Mechanical Systems and Signal Processing 11 (1997) 3-22 (Sections 1, 2.1, 3.4).

[42] A.H. Nayfeh, Introduction to Perturbation Techniques, Wiley-Interscience, New York, 1981 (Section 1).

[43] R.E. O'Malley, Singular Perturbation Methods for Ordinary Differential Equations, Springer, New York, 1991 (Section 1).

[44] J. Kevorkian, J.D. Cole, Multiple Scales and Singular Perturbation Methods, Springer, New York, 1996 (Section 1).

[45] H.S.Y. Chan, K.W. Chung, Z. Xu, A perturbation-incremental method for strongly non-linear oscillators, International Journal of Non-Linear Mechanics 31 (1996) 59-72 (Section 1).

[46] S.H. Chen, Y.K. Cheung, A modified Lindstedt-Poincaré method for a strongly nonlinear two degree-of-freedom system, Journal of Sound and Vibration 193 (1996) 751-762 (Section 1).

[47] V.N. Pilipchuk, The calculation of strongly nonlinear systems close to vibration-impact systems, PMM 49 (1985) 572-578 (Section 1).

[48] L.I. Manevitch, Complex Representation of Dynamics of Coupled Oscillators in Mathematical Models of Nonlinear Excitations, Transfer Dynamics and Control in Condensed Systems, Kluwer Academic/Plenum Publishers, New York, 1999 (Section 1). 
[49] M.I. Qaisi, A.W. Kilani, A power-series solution for a strongly non-linear two-degree-of-freedom system, Journal of Sound and Vibration 233 (2000) 489-494 (Section 1).

[50] J.F. Rhoads, S.W. Shaw, K.L. Turner, R. Baskaran, Tunable MEMS filters that exploit parametric resonance, Journal of Vibration and Acoustics (2005), in press (Section 1).

[51] A.F. Vakakis, O. Gendelman, Energy pumping in nonlinear mechanical oscillators: Part II—resonance capture, Journal of Applied Mechanics 68 (2001) 42-48 (Sections 1, 2.1, 8).

[52] A.F. Vakakis, D.M. McFarland, L.A. Bergman, L.I. Manevitch, O. Gendelman, Isolated resonance captures and resonance capture cascades leading to single- or multi-mode passive energy pumping in damped coupled oscillators, Journal of Vibration and Acoustics 126 (2004) 235-244 (Sections 1, 2.1).

[53] G. Kerschen, Y.S. Lee, A.F. Vakakis, D.M. McFarland, L.A. Bergman, Irreversible passive energy transfer in coupled oscillators with essential nonlinearity, SIAM Journal on Applied Mathematics (2005), in press (Sections 1, 2.1, 2.2).

[54] J.M. Nichols, C.J. Nichols, M.D. Todd, M. Seaver, S.T. Trickey, L.N. Virgin, Use of data-driven phase space models in assessing the strength of a bolted connection in a composite beam, Smart Materials and Structures 13 (2004) 241-250 (Section 1).

[55] B.I. Epureanu, A. Hashmi, Parameter reconstruction based on sensitivity vector fields, Journal of Vibration and Acoustics (2005), submitted for publication (Section 1).

[56] D.E. Adams, R.J. Allemang, Survey of nonlinear detection and identification techniques for experimental vibrations structural dynamic model through feedback, in: Proceedings of the International Seminar on Modal Analysis (ISMA), Leuven, 1998, pp. 269-281 (Sections 1, 4.1; Introduction Section 3).

[57] K. Worden, Nonlinearity in structural dynamics: the last ten years, in: Proceedings of the European COST F3 Conference on System Identification and Structural Health Monitoring, Madrid, 2000, pp. 29-52 (Section 1; Introduction Section 3).

[58] G. Duffing, Erzwungene Schwingungen bei Veranderlicher Eigenfrequenz (Forced Oscillations in the Presence of Variable Eigenfrequencies), Vieweg, Braunschweig, 1918 (Section 1).

[59] P.J. Roache, Verification and Validation in Computational Science and Engineering, Hermosa Publications, Albuquerque, 1998 (Sections 1, 7.4).

[60] S. Doebling, Structural dynamics model validation: pushing the envelope, in: Proceedings of International Conference on Structural Dynamics Modelling, Funchal, 2002 (Section 1).

[61] S. Schlesinger, R.E. Crosbie, R.E. Gagne, G.S. Innis, C.S. Lalwani, J. Loch, R.J. Sylvester, R.D. Wright, N. Kheir, D. Bartos, Terminology for model credibility, Simulation 32 (1979) 103-104 (Section 1).

[62] M. Link, M.I. Friswell, Working group 1: Generation of validated structural dynamic models — results of a benchmark study utilising the GARTEUR SM-AG19 test bed, Mechanical Systems and Signal Processing 17 (2003) 9-20 (Section 1).

[63] I. Babuska, J.T. Oden, Verification and validation in computational engineering and science: basic concepts, Computer Methods in Applied Mechanics and Engineering 193 (2004) 4057-4066 (Section 1).

[64] F.M. Hemez, S.W. Doebling, M.C. Anderson, Model Verification and Validation in Engineering Mechanics: Theory and Applications of Uncertainty Quantification and Predictive Accuracy, Wiley, New York, 2005 (Sections 1, 3.7, 8).

[65] I.J. Leontaritis, S.A. Billings, Experimental design and identifiability for non-linear systems, International Journal of Systems Science 18 (1987) 189-202 (Section 1).

[66] S. Duym, J. Schoukens, Design of excitation signals for the restoring force surface method, Mechanical Systems and Signal Processing 9 (1995) 139-158 (Sections 1, 3.2, 6.1).

[67] K. Worden, G.R. Tomlinson, Nonlinearity in Structural Dynamics: Detection, Identification and Modelling, Institute of Physics Publishing, Bristol and Philadelphia, 2001 (Sections 1, 4.1, 4.2, 5.1; Introduction Section 3).

[68] F. Moon, Chaotic Vibrations, Wiley Interscience, New York, 1987 (Section 1).

[69] M.A.F. Azeez, A.F. Vakakis, Numerical and experimental analysis of a continuously overhung rotor undergoing vibro-impacts, International Journal of Non-Linear Mechanics 34 (1999) 415-435 (Section 2.1).

[70] A. Jackson, Perspectives of Nonlinear Dynamics, Cambridge University Press, Cambridge, 1990 (Section 2.1).

[71] W.F. Ames, Nonlinear superposition for operator equations, in: V. Lakshmikanthan (Ed.), Nonlinear Equations in Abstract Spaces, Academic Press, New York, 1978 (Section 2.1).

[72] R.L. Anderson, J. Harnad, P. Winternitz, Systems of ordinary differential equations with nonlinear superposition techniques, Physica D 4 (1982) 164-182 (Section 2.1).

[73] V.A. Yakubovich, V.M. Starzhinskii, Linear Differential Equations with Periodic Coefficients I \& II, Wiley, New York, 1975 (Section 2.1).

[74] A.F. Vakakis, L.I. Manevitch, A. Musienko, G. Kerschen, L.A. Bergman, Transient dynamics of a dispersive elastic wave guide weakly coupled to an essentially nonlinear end attachment, Wave Motion 41 (2004) 109-132 (Section 2.1).

[75] N. Minorsky, Nonlinear Oscillations, Krieger Publishing Co., Florida, 1983 (Section 2.1).

[76] D. Panayotounakos, A. Panayotounakou, A.F. Vakakis, On the solution of the unforced duffing oscillator with damping, Nonlinear Dynamics 28 (2002) 1-16 (Section 2.1).

[77] M.E. King, A.F. Vakakis, An energy-based approach to computing resonant nonlinear normal modes, Journal of Applied Mechanics 63 (1996) 810-819 (Section 2.1).

[78] S.W. Shaw, C. Pierre, Non-linear normal modes and invariant manifolds, Journal of Sound and Vibration 150 (1991) 170-173 (Section 2.1)

[79] A.H. Nayfeh, S.A. Nayfeh, On nonlinear modes of continuous systems, Journal of Vibration and Acoustics 116 (1994) 129-136 (Section 2.1). 
[80] A.H. Nayfeh, S.A. Nayfeh, Nonlinear normal modes of a continuous system with quadratic nonlinearities, Journal of Vibration and Acoustics 117 (1995) 199-205 (Section 2.1).

[81] S. Shaw, P.J. Holmes, A periodically forced linear oscillator with impacts: chaos and long period motions, Physical Review Letters 51 (1983) 623-626 (Section 2.1).

[82] F. Moon, P.H. Holmes, A magnetoelastic strange attractor, Journal of Sound and Vibration 65 (1979) 285-296 (Section 2.1).

[83] P. Holmes, The dynamics of repeated impacts with a sinusoidally vibrating table, Journal of Sound and Vibration 84 (1982) 173-189 (Section 2.1).

[84] A.F. Vakakis, R.H. Rand, Normal modes and global dynamics of a two degree-of-freedom nonlinear system, ii: high energies, International Journal of Non-Linear Mechanics 27 (1992) 875-888 (Section 2.1).

[85] I.B. Schwartz, D.S. Morgan, L. Billings, Y.C. Lai, Multi-scale continuum mechanics: from global bifurcations to noise induced high-dimensional chaos, Chaos 14 (2004) 373-386 (Section 2.1).

[86] J.P. Cusumano, M.T. Sharkady, B.W. Kimble, Experimental measurements of dimensionality and spatial coherence in the dynamics of a flexible-beam impact oscillator, Philosophical Transactions of the Royal Society of London 347 (1994) 421 -438 (Section 2.1).

[87] M.F.A. Azeez, A.F. Vakakis, Proper orthogonal decomposition of a class of vibroimpact oscillations, Journal of Sound and Vibration 240 (2001) 859-889 (Section 2.1).

[88] R.S. MacKay, J.D. Meiss, Hamiltonian Dynamical Systems: A Reprint Selection, Adam Hilger, Bristol, Philadelphia, 1987 (Section 2.1).

[89] S.F. Dermott, C.D. Murray, Nature of the Kirkwood gaps in the asteroid belt, Nature 301 (1983) 201-205 (Section 2.1).

[90] J. Wisdom, S.J. Peale, F. Mignard, The chaotic rotation of hyperion, Icarus 58 (1984) 137-152 (Section 2.1).

[91] A. Gerasimov, F.M. Izrailev, J.L. Tennyson, A.B. Temnykh, The dynamics of the beam-beam interaction, Springer Lecture Notes in Physics, vol. 247, 1986, pp. 154-175 (Section 2.1).

[92] S. Wiggins, Chaotic Transport in Dynamical Systems, Springer, Berlin, 1992 (Section 2.1).

[93] A.H. Nayfeh, D.T. Mook, Energy transfer from high-frequency to low-frequency modes in structures, Journal of Vibration and Acoustics 117 (1995) 186-195 (Section 2.1).

[94] P. Malatkar, A.H. Nayfeh, On the transfer of energy between widely spaced modes in structures, Nonlinear Dynamics 31 (2003) 225-242 (Section 2.1).

[95] D. Quinn, Resonance capture in a three degree of freedom mechanical system, Nonlinear Dynamics 14 (1997) 309-333 (Sections 2.1, $2.2,8)$.

[96] A. Zniber, D. Quinn, Frequency shifting in nonlinear resonant systems with damping, Proceedings of the 2003 ASME Design Engineering Technical Conferences, Chicago, DETC2003/VIB-48444 (Section 2.1).

[97] Y.S. Lee, G. Kerschen, A.F. Vakakis, P.N. Panagopoulos, L.A. Bergman, D.M. McFarland, Complicated dynamics of a linear oscillator with a light, essentially nonlinear attachment, Physica D 204 (2005) 41-69 (Sections 2.1, 2.2).

[98] A. Tondl, T. Ruijgrok, F. Verhulst, R. Nabergoj, Autoparametric Resonances in Mechanical Systems, Cambridge University Press, Cambridge, 2000 (Section 2.1).

[99] V.I. Arnold, Dynamical Systems III, Encyclopedia of Mathematical Sciences, Springer, Berlin, 1988 (Section 2.2).

[100] P. Ibanez, Identification of dynamic parameters of linear and non-linear structural models from experimental data, Nuclear Engineering and Design 25 (1973) 30-41 (Introduction Section 3).

[101] S.F. Masri, T.K. Caughey, A nonparametric identification technique for nonlinear dynamic problems, Journal of Applied Mechanics 46 (1979) 433-447 (Introduction Section 3; Sections 3.2, 5.1, 6.1, 6.2).

[102] F.M. Hemez, S.W. Doebling, Inversion of structural dynamics simulations: state-of-the-art and orientations of the research, in: Proceedings of the International Seminar on Modal Analysis (ISMA), Leuven, 2000 (Introduction Section 3; Section 8).

[103] F.M. Hemez, S.W. Doebling, Review and assessment of model updating for non-linear, transient dynamics, Mechanical Systems and Signal Processing 15 (2001) 45-74 (Introduction Section 3; Sections 3.4, 3.7, 7.4, 8).

[104] J.C. Golinval, G. Kerschen, V. Lenaerts, F. Thouverez, P. Argoul, European COST action F3 on structural dynamics. Working group 3: identification of non-linear systems; Introduction and conclusions, Mechanical Systems and Signal Processing 17 (2003) 177-178, 251-254 (Introduction Section 3; Sections 7.1, 7.4).

[105] F. Thouverez, Presentation of the ECL benchmark, Mechanical Systems and Signal Processing 17 (2003) 195-202 (Introduction Section 3).

[106] E.H. Dowell, B.I. Epureanu, Preface, Nonlinear Dynamics 39 (2005) 1 (Introduction Section 3).

[107] R. Singh, in: K. Worden, G.R. Tomlinson (Eds.), Nonlinearity in Structural Dynamics: Detection, Identification and Modelling, Journal of Sound and Vibration 270 (2004) 1087-1088 (Introduction Section 3).

[108] J.S. Bendat, Nonlinear System Techniques and Applications, Wiley, New York, 1998 (Introduction Section 3; Sections 3.3, 6.6).

[109] J.S. Bendat, A.G. Piersol, Random Data: Analysis and Measurement Procedures, third ed., Wiley Interscience, New York, 2000 (Introduction Section 3; Sections 5.1, 7.1).

[110] T.K. Caughey, Response of Van der Pol's oscillator to random excitations, Journal of Applied Mechanics 26 (1959) 345-348 (Section 3.1).

[111] T.K. Caughey, Random excitation of a system with bilinear hysteresis, Journal of Applied Mechanics 27 (1960) 649-652 (Section 3.1).

[112] I.E. Kazakov, Approximate probabilistic analysis of the accuracy of operation of essentially nonlinear systems, Automatika i Telemekhanika 17 (1956) 423-450 (Section 3.1).

[113] L. Socha, M. Pawleta, Are statistical linearization and standard equivalent linearization the same methods in the analysis of stochastic dynamic systems?, Journal of Sound and Vibration 248 (2001) 387-394 (Section 3.1). 
[114] W.D. Iwan, A.B. Mason, Equivalent linearization for systems subjected to non-stationary random excitation, International Journal of Non-linear Mechanics 15 (1980) 71-82 (Section 3.1).

[115] J.B. Roberts, P.D. Spanos, Random Vibrations and Statistical Linearization, Wiley, New York, 1990 (Section 3.1 ).

[116] P. Hagedorn, J. Wallaschek, On equivalent harmonic and stochastic linearization, in: Proceedings of the IUTAM Symposium on Nonlinear Stochastic Dynamic Engineering Systems, Berlin, 1987, pp. 23-32 (Section 3.1).

[117] O. Fillatre, Identification of weakly nonlinear dynamic systems by means of random excitations, La Recherche Aérospatiale 3 (1992) 11-22 (Section 3.1).

[118] H.J. Rice, Identification of weakly non-linear systems using equivalent linearization, Journal of Sound and Vibration 185 (1995) 473-481 (Section 3.1).

[119] R.N. Miles, An approximate solution for the spectral response of Duffing's oscillator with random input, Journal of Sound and Vibration 132 (1989) 43-49 (Section 3.1).

[120] S.H. Crandall, Les vibrations forcées dans les systèmes non-linéaires, Colloques Internationaux du CNRS, Marseille, 1963 (Section 3.1).

[121] R. Bouc, The power spectral density of response for a strongly non-linear random oscillator, Journal of Sound and Vibration 175 (1994) 317-331 (Section 3.1).

[122] C. Soize, Stochastic linearization method with random parameters and power spectral density calculation, in: Proceedings of the International Conference on Structural Safety and Reliability, Rotterdam, 1994 (Section 3.1).

[123] C. Soize, Stochastic linearization method with random parameters for SDOF nonlinear dynamical systems: prediction and identification procedures, Probabilistic Engineering Mechanics 10 (1995) 143-152 (Section 3.1).

[124] C. Soize, O. Le Fur, Modal identification of weakly non-linear multidimensional dynamical systems using a stochastic linearisation method with random coefficients, Mechanical Systems and Signal Processing 11 (1997) 37-49 (Section 3.1).

[125] S. Bellizzi, R. Bouc, M. Defilippi, P. Guihot, Response spectral densities and identification of a randomly excited non-linear squeeze film oscillator, Mechanical Systems and Signal Processing 12 (1998) 693-711 (Section 3.1).

[126] S. Bellizzi, M. Defilippi, Non-linear mechanical systems identification using linear systems with random parameters, Mechanical Systems and Signal Processing 17 (2003) 203-210 (Section 3.1).

[127] K. Yasuda, S. Kawamura, K. Watanabe, Identification of nonlinear multi-degree-of-freedom systems (presentation of an identification technique), JSME International Journal Series III 31 (1988) 8-14 (Sections 3.1, 3.3).

[128] K. Yasuda, S. Kawamura, K. Watanabe, Identification of nonlinear multi-degree-of-freedom systems (identification under noisy measurements), JSME International Journal Series III 31 (1988) 302-309 (Sections 3.1, 3.3).

[129] Y. Benhafsi, J.E. Penny, M.I. Friswell, A parameter identification method for discrete nonlinear systems incorporating cubic stiffness elements, International Journal of Analytical and Experimental Modal Analysis 7 (1992) 179-195 (Section 3.1).

[130] S. Meyer, M. Weiland, M. Link, Modelling and updating of local non-linearities using frequency response residuals, Mechanical Systems and Signal Processing 17 (2003) 219-226 (Sections 3.1, 3.7).

[131] M.E. Ozer, H.N. Ozgüven, T.J. Royston, Identification of structural non-linearities using describing functions and Sherman-Morrison method, in: Proceedings of the 23rd International Modal Analysis Conference, Orlando, 2005 (Section 3.1).

[132] E.F. Crawley, K.J. O’Donnell, Identification of nonlinear system parameters in joints using the force-state mapping technique, AIAA Paper 86-1013 (1986) 659-667 (Sections 3.2, 6.1).

[133] E.F. Crawley, A.C. Aubert, Identification of nonlinear structural elements by force-state mapping, AIAA Journal 24 (1986) $155-162$ (Sections 3.2, 6.1).

[134] S.F. Masri, H. Sassi, T.K. Caughey, A nonparametric identification of nearly arbitrary nonlinear systems, Journal of Applied Mechanics 49 (1982) 619-628 (Sections 3.2, 3.4, 6.2).

[135] Y. Yang, S.R. Ibrahim, A nonparametric identification technique for a variety of discrete nonlinear vibrating systems, Journal of Vibration, Acoustics, Stress, and Reliability in Design 107 (1985) 60-66 (Sections 3.2, 6.2).

[136] S.F. Masri, R.K. Miller, A.F. Saud, T.K. Caughey, Identification of nonlinear vibrating structures: part I-formalism, Journal of Applied Mechanics 54 (1987) 918-922 (Sections 3.2, 6.2).

[137] S.F. Masri, R.K. Miller, A.F. Saud, T.K. Caughey, Identification of nonlinear vibrating structures: part II-applications, Journal of Applied Mechanics 54 (1987) 923-929 (Sections 3.2, 6.2).

[138] M. A Al-Hadid, J.R. Wright, Developments in the force-state mapping technique for non-linear systems and the extension to the location of non-linear elements in a lumped-parameter system, Mechanical Systems and Signal Processing 3 (1989) 269-290 (Sections 3.2, 3.4, 5.1,6.2).

[139] M. A Al-Hadid, J.R. Wright, Application of the force-state mapping approach to the identification of non-linear systems, Mechanical Systems and Signal Processing 4 (1990) 463-482 (Sections 3.2, 6.2).

[140] M. A Al-Hadid, J.R. Wright, Estimation of mass and modal mass in the identification of nonlinear single and multi DOF systems using the force-state mapping approach, Mechanical Systems and Signal Processing 6 (1992) 383-401 (Sections 3.2, 6.2).

[141] K. Worden, Data processing and experiment design for the restoring force surface method, Part I: integration and differentiation of measured time data, Mechanical Systems and Signal Processing 4 (1990) 295-319 (Sections 3.2, 6.1).

[142] K. Worden, Data processing and experiment design for the restoring force surface method, Part II: choice of excitation signal, Mechanical Systems and Signal Processing 4 (1990) 321-344 (Sections 3.2, 6.1).

[143] K.S. Mohammad, K. Worden, G.R. Tomlinson, Direct parameter estimation for linear and nonlinear structures, Journal of Sound and Vibration 152 (1991) 471-499 (Sections 3.2, 6.2).

[144] K. Shin, J.K. Hammond, Pseudo force-state mapping method: incorporation of the embedding and force-state mapping methods, Journal of Sound and Vibration 211 (1998) 918-922 (Sections 3.2, 6.1). 
[145] W.J. Kim, S.J. Park, Non-linear joint parameter identification by applying the force-state mapping technique in the frequency domain, Mechanical System and Signal Processing 8 (1994) 519-529 (Section 3.2).

[146] H.R. Lo, J.K. Hammond, Identification of a class of nonlinear systems, preprint, Institute of Sound and Vibration Research, Southampton, 1988 (Sections 3.2, 6.1).

[147] F. Benedettini, D. Capecchi, F. Vestroni, Nonparametric models in identification of hysteretic oscillators, Report DISAT N.4190, Dipartimento di Ingegneria delle Strutture, Universita' dell'Aquila, Italy, 1991 (Sections 3.2, 6.1).

[148] K. Shin, J.K. Hammond, Force-state mapping method of a chaotic dynamical system, Journal of Sound and Vibration 218 (1998) 405-418 (Section 3.2).

[149] A. Audenino, G. Belingardi, L. Garibaldi, An application of the restoring force mapping method for the diagnostic of vehicular shock absorbers dynamic behaviour, preprint, Dipartimento di Meccanica del Politecnico di Torino, 1990 (Sections 3.2, 6.1).

[150] G. Belingardi, P. Campanile, Improvement of the shock absorber dynamic simulation by the restoring force mapping method, in: Proceedings of the International Seminar in Modal Analysis and Structural Dynamics, Leuven, 1990 (Sections 3.2, 5.2, 6.1).

[151] C. Surace, K. Worden, G.R. Tomlinson, On the nonlinear characteristics of automotive shock absorbers, Proceedings of the I. Mech. E., Part D-Journal of Automobile Engineering 206 (1992) 3-16 (Sections 3.2, 5.2, 6.1).

[152] S. Cafferty, K. Worden, G.R. Tomlinson, Characterisation of automotive shock absorbers using random excitation, Proceedings of the I. Mech. E., Part D-Journal of Automobile Engineering 209 (1993) 239-248 (Section 3.2).

[153] S. Duym, R. Stiens, K. Reybrouck, Fast parametric and nonparametric identification of shock absorbers, Proceedings of the International Seminar on Modal Analysis (ISMA), Leuven, 1996 (Section 3.2).

[154] S. Duym, J. Schoukens, P. Guillaume, A local restoring force surface method, International Journal of Analytical and Experimental Modal Analysis 11 (1996) 116-132 (Section 3.2, 6.1).

[155] S. Duym, J. Schoukens, Selection of an optimal force-state map, Mechanical Systems and Signal Processing 10 (1996) 683-695 (Section 3.2).

[156] G. Kerschen, J.C. Golinval, K. Worden, Theoretical and experimental identification of a non-linear beam, Journal of Sound and Vibration 244 (2001) 597-613 (Sections 3.2, 6.1).

[157] C. Meskell, J.A. Fitzpatrick, H.J. Rice, Application of force-state mapping to a non-linear fluid-elastic system, Mechanical Systems and Signal Processing 15 (2001) 75-85 (Sections 3.2, 6.1).

[158] G. Dimitriadis, J.E. Cooper, A method for the identification of non-linear multi-degree-of-freedom systems, Proceedings of the Institute of Mechanical Engineers, Part G 212 (1998) 287-298 (Section 3.2).

[159] M. Haroon, D.E. Adams, Y.W. Luk, A technique for estimating linear parameters of an automotive suspension system using nonlinear restoring force excitation, ASME International Mechanical Engineering Congress, Anaheim, 2004 (Section 3.2).

[160] M. Haroon, D.E. Adams, Y.W. Luk, A.A. Ferri, A time and frequency domain approach for identifying nonlinear mechanical system models in the absence of an input measurement, Journal of Sound and Vibration 283 (2005) 1137-1155 (Sections 3.2, 3.3, 8).

[161] G.E.P. Box, G.M. Jenkins, Time Series Analysis, Forecasting and Control, Holden-Day, San Francisco, 1970 (Section 3.2).

[162] I.J. Leontaritis, S.A. Billings, Input-output parametric models for nonlinear systems, part I: deterministic nonlinear systems, International Journal of Control 41 (1985) 303-328 (Sections 3.2, 6.3).

[163] I.J. Leontaritis, S.A. Billings, Input-output parametric models for nonlinear systems, part II: stochastic nonlinear systems, International Journal of Control 41 (1985) 329-344 (Sections 3.2, 6.3).

[164] M. Korenberg, S.A. Billings, Y.P. Liu, P.J. McIlroy, An orthogonal parameter estimation algorithm for nonlinear stochastic systems, International Journal of Control 48 (1988) 193-210 (Sections 3.2, 6.3).

[165] S.A. Billings, S. Chen, R.J. Backhouse, Identification of linear and nonlinear models of a turbocharged automotive diesel engine, Mechanical Systems and Signal Processing 3 (1989) 123-142 (Sections 3.2, 6.3).

[166] S.A. Billings, H.B. Jamaluddin, S. Chen, Properties of neural networks with applications to modelling non-linear dynamical systems, International Journal of Control 55 (1991) 193-224 (Sections 3.2, 3.6, 6.3).

[167] S. Chen, S.A. Billings, C.F.N. Cowan, P.M. Grant, Practical identification of NARMAX models using radial basis functions, International Journal of Control 52 (1990) 1327-1350 (Section 3.2).

[168] E. Bedrosian, S.O. Rice, The output properties of Volterra systems driven by harmonic and Gaussian inputs, Proceedings IEEE 59 (1971) 1688-1707 (Sections 3.2, 6.5).

[169] S.A. Billings, K.M. Tsang, Spectral analysis for nonlinear systems, part I: parametric non-linear spectral analysis, Mechanical Systems and Signal Processing 3 (1989) 319-339 (Section 3.2).

[170] S.A. Billings, K.M. Tsang, Spectral analysis for nonlinear systems, part II: interpretation of nonlinear frequency response functions, Mechanical Systems and Signal Processing 3 (1989) 341-359 (Sections 3.2, 6.5).

[171] F. Thouverez, L. Jezequel, Identification of NARMAX models on a modal base, Journal of Sound and Vibration 89 (1996) 193-213 (Sections 3.2, 6.3).

[172] M. Feldman, Nonlinear system vibration analysis using the Hilbert transform-I. Free vibration analysis method 'FREEVIB', Mechanical Systems and Signal Processing 8 (1994) 119-127 (Sections 3.2, 4.1, 6.4).

[173] M. Feldman, Nonlinear system vibration analysis using the Hilbert transform-I. Forced vibration analysis method 'FORCEVIB', Mechanical Systems and Signal Processing 8 (1994) 309-318 (Sections 3.2, 6.4).

[174] O. Gottlieb, M. Feldman, S.C.S. Yim, Parameter identification of nonlinear ocean mooring systems using the Hilbert transform, Journal of Offshore Mechanics and Arctic Engineering 118 (1996) 29-36 (Section 3.2).

[175] M. Feldman, Non-linear free vibration identification via the Hilbert transform, Journal of Sound and Vibration 208 (1997) 475-489 (Sections 3.2, 6.4). 
[176] N.E. Huang, Z. Shen, S.R. Long, M.C. Wu, H.H. Shih, Q. Zheng, N.C. Yen, C.C. Tung, H.H. Liu, The empirical mode decomposition and the Hilbert spectrum for nonlinear and non-stationary time series analysis, in: Proceedings of the Royal Society of London Series A-Mathematical, Physical and Engineering Sciences 454 (1998) 903-995. (Sections 3.2, 4.1, 6.4)

[177] J.N. Yang, Y. Lei, S.W. Pan, N. Huang, System identification of linear structures based on Hilbert-Huang spectral analysis; Part 1: Normal modes, Earthquake Engineering and Structural Dynamics 32 (2003) 1443-1467 (Section 3.2).

[178] J.N. Yang, Y. Lei, S.W. Pan, N. Huang, System identification of linear structures based on Hilbert-Huang spectral analysis; Part 2: Complex modes, Earthquake Engineering and Structural Dynamics 32 (2003) 1533-1554 (Section 3.2).

[179] J.N. Yang, S. Lin, Hilbert-Huang based approach for structural damage detection, Journal of Engineering Mechanics 130 (2004) 85-95 (Section 3.2).

[180] S.L. Lacy, D.S. Bernstein, Subspace identification for nonlinear systems that are linear in unmeasured states, Proceedings of the 40th IEEE Conference on Decision and Control, Orlando, 2001, pp. 3518-3523 (Section 3.2).

[181] V.N. Pilipchuk, C.M. Tan, Non-linear system identification based on the Lie series solutions, Mechanical Systems and Signal Processing 19 (2005) 71-86 (Section 3.2).

[182] B.F. Feeny, J.W. Liang, A decrement method for the simultaneous estimation of coulomb and viscous friction, Journal of Sound and Vibration 195 (1996) 149-154 (Section 3.2).

[183] J.W. Liang, B.F. Feeny, Identifying Coulomb and viscous friction from free-vibration decrements, Nonlinear Dynamics 16 (1998) 337-347 (Section 3.2).

[184] J.W. Liang, B.F. Feeny, Identifying Coulomb and viscous friction in forced dual-damped oscillators, Journal of Vibration and Acoustics 126 (2004) 118-125 (Section 3.2).

[185] R. Singh, P. Davies, A.K. Bajaj, Initial condition response of a viscoelastic dynamical system in the presence of dry friction and identification of system parameters, Journal of Sound and Vibration 239 (2001) 1086-1095 (Section 3.2).

[186] A. Chatterjee, J.P. Cusumano, Asymptotic parameter estimation via implicit averaging on a nonlinear extended system, Journal of Dynamic Systems, Measurement, and Control 125 (2003) 11-18 (Section 3.2).

[187] K. Yasuda, K. Kamiya, Experimental identification technique of nonlinear beams in time domain, Nonlinear Dynamics 18 (1999) 185-202 (Section 3.2).

[188] M. Schetzen, The Volterra and Wiener Theories of Nonlinear Systems, Wiley, New York, 1980 (Sections 3.3, 6.5).

[189] F. Thouverez, L. Jezequel, Identification of a localized non-linearity, International Journal of Non-Linear Mechanics 33 (1998) 935-945 (Section 3.3).

[190] S.J. Gifford, Volterra series analysis of nonlinear structures, Ph.D. Thesis, Department of Mechanical Engineering, Heriot-Watt University, 1989 (Sections 3.3, 6.5).

[191] D.M. Storer, Dynamic analysis of nonlinear structures using higher order frequency response functions, Ph.D. Thesis, Department of Engineering, University of Manchester, 1991 (Sections 3.3, 6.5).

[192] D.M. Storer, G.R. Tomlinson, Recent developments in the measurements and interpretation of higher order functions from nonlinear structures, Mechanical Systems and Signal Processing 7 (1993) 173-189 (Sections 3.3, 4.1, 5.1).

[193] A.A. Khan, N.S. Vyas, Non-linear parameter using Volterra and Wiener theories, Journal of Sound and Vibration 221 (1999) 805-821 (Sections 3.3, 6.5).

[194] A. Chatterjee, N.S. Vyas, Non-linear parameter estimation through Volterra series using the method of recursive iteration through harmonic probing, Journal of Sound and Vibration 268 (2003) 657-678 (Sections 3.3, 6.5).

[195] A.A. Khan, N.S. Vyas, Nonlinear bearing stiffness parameter estimation in flexible rotor-bearing systems using Volterra and Wiener approach, Probabilistic Engineering Mechanics 16 (2001) 137-157 (Section 3.3).

[196] A. Chatterjee, N.S. Vyas, Non-linear parameter estimation in multi-degree-of-freedom systems using multi-input Volterra series, Mechanical Systems and Signal Processing 18 (2004) 457-489 (Sections 3.3, 6.5).

[197] A.A. Khan, N.S. Vyas, Application of Volterra and Wiener theories for nonlinear parameter estimation in a rotor-bearing system, Nonlinear Dynamics 24 (2001) 285-304 (Section 3.3).

[198] I. Tawfiq, T. Vinh, Contribution to the extension of modal analysis to non-linear structure using Volterra functional series, Mechanical Systems and Signal Processing 17 (2003) 379-407 (Sections 3.3, 6.5).

[199] I. Tawfiq, T. Vinh, Nonlinear behaviour of structures using the Volterra series signal processing and testing methods, Nonlinear Dynamics 37 (2004) 129-149 (Sections 3.3, 6.5).

[200] J.B. Roberts, J.F. Dunne, A. Debonos, A spectral method for estimation of non-linear system parameters from measured response, Probabilistic Engineering Mechanics 10 (1995) 199-207 (Section 3.3).

[201] M. Vasta, J.B. Roberts, Stochastic parameter estimation of non-linear systems using only higher order spectra of the measured response, Journal of Sound and Vibration 213 (1998) 201-221 (Section 3.3).

[202] J.B. Roberts, M. Vasta, Parametric identification of systems with non-Gaussian excitation using measured response spectra, Probabilistic Engineering Mechanics 15 (2000) 59-71 (Section 3.3).

[203] J.B. Roberts, M. Vasta, Energy-based stochastic estimation for nonlinear oscillators with random excitation, Journal of Applied Mechanics 67 (2000) 763-771 (Section 3.3).

[204] M.R. Hajj, J. Fung, A.H. Nayfeh, S. Fahey, Damping identification using perturbation techniques and higher-order spectra, Nonlinear Dynamics 23 (2000) 189-203 (Sections 3.3, 5.1).

[205] A. Swami, G.B. Giannakis, G. Zhou, Bibliography on higher-order statistics, Signal Processing 60 (1997) 65-126 (Section 3.3).

[206] K. Yasuda, K. Kamiya, Identification of a nonlinear beam proposition of an identification technique, JSME International Journal Series III 33 (1990) 535-540 (Section 3.3). 
[207] K. Yasuda, K. Kamiya, Experimental identification technique of vibrating structures with geometrical nonlinearity, Journal of Applied Mechanics 64 (1997) 275-280 (Section 3.3).

[208] C.M. Yuan, B.F. Feeny, Parametric identification of chaotic systems, Journal of Vibration and Control 4 (1998) 405-426 (Section 3.3).

[209] B.F. Feeny, C.M. Yuan, J.P. Cusumano, Parametric identification of an experimental magneto-elastic oscillator, Journal of Sound and Vibration 247 (2001) 785-806 (Section 3.3).

[210] Y. Liang, B.F. Feeny, Parametric identification of chaotic base-excited double pendulum experiment, ASME International Mechanical Engineering Congress, Anaheim, 2004 (Section 3.3).

[211] M. Thothadrai, R.A. Casas, F.C. Moon, R. D'Andrea, C.R. Johnson, Nonlinear system identification of multi-degree-of-freedom systems, Nonlinear Dynamics 32 (2003) 307-322 (Section 3.3).

[212] M. Thothadrai, F.C. Moon, Nonlinear system identification of systems with periodic limit-cycle response, Nonlinear Dynamics 39 (2005) (Section 3.3).

[213] H.J. Rice, J.A. Fitzpatrick, A generalised technique for spectral analysis of non-linear systems, Mechanical Systems and Signal Processing 2 (1988) 195-207 (Sections 3.3, 6.6).

[214] H. Esmonde, J.A. Fitzpatrick, H.J. Rice, F. Axisa, Analysis of non-linear squeeze film dynamics: part I-physical theory and modelling, Proceedings of ASME PVP Conference, Nashville, 1990 (Section 3.3)

[215] H. Esmonde, F. Axisa, J.A. Fitzpatrick, H.J. Rice, Analysis of non-linear squeeze film dynamics: part II-experimental measurement and model verification, Proceedings of ASME PVP Conference, Nashville, 1990 (Section 3.3).

[216] J.S. Bendat, Nonlinear System Analysis and Identification from Random Data, Wiley, New York, 1990 (Sections 3.3, 6.6).

[217] H.J. Rice, J.A. Fitzpatrick, The measurement of nonlinear damping in single-degree-of-freedom systems, Journal of Vibration and Acoustics 113 (1991) 132-140 (Section 3.3).

[218] J.S. Bendat, R.N. Coppolino, P.A. Palo, Identification of physical parameters with memory in non-linear systems, International Journal of Non-Linear Mechanics 30 (1995) 841-860 (Section 3.3).

[219] B.A. Zeldin, P.D. Spanos, Spectral identification of nonlinear structures, Journal of Engineering Mechanics 124 (1998) $728-733$ (Section 3.3).

[220] J.S. Bendat, Spectral techniques for nonlinear system analysis and identification, Shock and Vibration 1 (1993) 21-31 (Section 3.3).

[221] H.J. Rice, J.A. Fitzpatrick, A procedure for the identification of linear and non-linear multi-degree-of-freedom systems, Journal of Sound and Vibration 149 (1991) 397-411 (Sections 3.3, 6.6).

[222] C.M. Richards, R. Singh, Identification of multi-degree-of-freedom non-linear systems under random excitations by the reverse-path spectral method, Journal of Sound and Vibration 213 (1998) 673-708 (Sections 3.3, 6.6, 7.1).

[223] G. Kerschen, J.C. Golinval, Generation of accurate finite element models of nonlinear systems - application to an aeroplane-like structure, Nonlinear Dynamics 39 (2005) 129-142 (Sections 3.3, 3.7, 7.1).

[224] J.A. Fitzpatrick, H.J. Rice, Comments on "Identification of multi-degree-of-freedom non-linear systems under random excitations by the 'reverse path' spectral method", Journal of Sound and Vibration 237 (2000) 357-358 (Section 3.3).

[225] C.M. Richards, R. Singh, Comments on "Identification of multi-degree-of-freedom non-linear systems under random excitations by the 'reverse path' spectral method"-Authors' reply, Journal of Sound and Vibration 237 (2000) 358-360 (Section 3.3).

[226] C.M. Richards, R. Singh, Comparison of two non-linear system identification approaches derived from "reverse path" spectral analysis, Journal of Sound and Vibration 237 (2000) 361-376 (Section 3.3).

[227] C.M. Richards, R. Singh, Feasibility of identifying non-linear vibratory systems consisting of unknown polynomial forms, Journal of Sound and Vibration 220 (1999) 413-450 (Sections 3.3, 5.1).

[228] G. Kerschen, V. Lenaerts, S. Marchesiello, A. Fasana, A frequency domain versus a time domain identification technique for nonlinear parameters applied to wire rope isolators, Journal of Dynamic Systems, Measurement, and Control 123 (2001) 645-650 (Section 3.3).

[229] G. Kerschen, V. Lenaerts, J.C. Golinval, Identification of a continuous structure with a geometrical non-linearity, part I: conditioned reverse path method, Journal of Sound and Vibration 262 (2003) 889-906 (Sections 3.3, 5.1, 7.1).

[230] L. Garibaldi, Application of the conditioned reverse path method, Mechanical Systems and Signal Processing 17 (2003) $227-236$ (Section 3.3).

[231] S. Marchesiello, Application of the conditioned reverse path method, Mechanical Systems and Signal Processing 17 (2003) 183-188 (Section 3.3).

[232] D.E. Adams, R.J. Allemang, A frequency domain method for estimating the parameters of a non-linear structural dynamic model through feedback, Mechanical Systems and Signal Processing 14 (2000) 637-656 (Sections 3.3, 7.2).

[233] D.E. Adams, R.J. Allemang, A new derivation of the frequency response function matrix for nonlinear vibrating systems, Journal of Sound and Vibration 227 (1999) 1083-1108 (Section 3.3).

[234] D.E. Adams, R.J. Allemang, Characterization of nonlinear vibrating systems using internal feedback and frequency response modulation, Journal of Vibration and Acoustics 121 (1999) 495-500 (Sections 3.3, 5.1).

[235] J.A. Vazquez Feijoo, K. Worden, R. Stanway, System identification using associated linear equations, Mechanical Systems and Signal Processing 18 (2004) 431-455 (Section 3.3).

[236] J.X. Zhang, J.B. Roberts, A frequency domain parametric identification method for studying the non-linear performance of squeezefilm dampers, Journal of Sound and Vibration 189 (1996) 173-191 (Section 3.3).

[237] R.W. Krauss, A.H. Nayfeh, Experimental nonlinear identification of a single mode of a transversely excited beam, Nonlinear Dynamics 18 (1999) 69-87 (Section 3.3).

[238] P. Malatkar, A.H. Nayfeh, A parametric identification technique for single-degree-of-freedom weakly nonlinear systems with cubic nonlinearities, Journal of Vibration and Control 9 (2003) 317-336 (Section 3.3). 
[239] A.H. Nayfeh, Parametric identification of nonlinear dynamic systems, Computers and Structures 20 (1985) $487-493$ (Section 3.3).

[240] S. Fahey, A.H. Nayfeh, Experimental nonlinear identification of a single structural mode, in: Proceedings of the 16th International Modal Analysis Conference, Orlando, 1998, pp. 737-745 (Section 3.3).

[241] T.A. Doughty, P. Davies, A.K. Bajaj, A comparison of three techniques using steady state data to identify non-linear modal behavior of an externally excited cantilever beam, Journal of Sound and Vibration 249 (2002) 785-813 (Section 3.3).

[242] J.C. Golinval, M. Link, COST action F3 Structural Dynamics (1997-2001) - an European co-operation in the field of science and technology, Mechanical Systems and Signal Processing 17 (2003) 3-7 (Section 3.3).

[243] J. Piranda, R. Fillod, E. Foltete, Modal identification of non-linear structures, in: Proceedings of the International Seminar on Modal Analysis (ISMA), Leuven, 1998 (Section 3.4).

[244] D. Göge, U. Fullekrug, M. Link, L. Gaul, A strategy for the identification and characterisation of non-linearities within modal survey testing, in: Proceedings of the 22nd International Modal Analysis Conference, Dearborn, 2004 (Section 3.4).

[245] W. Szemplinska-Stupnicka, The modified single mode method in the investigations of the resonant vibrations of nonlinear systems, Journal of Sound and Vibration 65 (1979) 475-489 (Section 3.4).

[246] W. Szemplinska-Stupnicka, Nonlinear normal modes and generalized Ritz method in the problems of vibrations of nonlinear elastic continuous systems, International Journal of Non-Linear Mechanics 18 (1983) 149-165 (Section 3.4).

[247] L. Jezequel, Extension des méthodes de synthèse modale au cas non linéaire, Revue-francaise-de-Mecanique 3 (1987) 159-172 (Section 3.4).

[248] S. Setio, H.D. Setio, L. Jezequel, Modal analysis of non-linear multi-degree-of-freedom systems, International Journal of Analytical and Experimental Modal Analysis 7 (1992) 75-93 (Section 3.4).

[249] S. Setio, H.D. Setio, L. Jezequel, A method of nonlinear modal identification from frequency-response tests, Journal of Sound and Vibration 158 (1992) 497-515 (Section 3.4).

[250] Y.H. Chong, M. Imregun, Development and application of a nonlinear modal analysis technique for multi-degree-of-freedom systems, Journal of Vibration and Control 7 (2001) 167-179 (Section 3.4).

[251] C. Gibert, F. Thouverez, L. Jezequel, Non-linear modal analysis applied to an industrial structure, in: Proceedings of the 17th International Modal Analysis Conference, Kissimmee, 1999, pp. 87-93 (Section 3.4).

[252] C. Gibert, Fitting measured frequency response using non-linear modes, Mechanical Systems and Signal Processing 17 (2003) 211-218 (Section 3.4).

[253] L. Huang, W.D. Iwan, Modal identification of nonlinear systems using successive approximation model, in: Proceedings of the 15th International Modal Analysis Conference, Orlando, 1997 (Section 3.4).

[254] E. Pesheck, N. Boivin, C. Pierre, S.W. Shaw, Nonlinear modal analysis of structural systems using multi-mode invariant manifolds, Nonlinear Dynamics 25 (2001) 183-205 (Sections 3.4, 8).

[255] J.R. Wright, M.F. Platten, J.E. Cooper, M. Sarmast, Identification of multi-degree-of-freedom weakly non-linear systems using a model based in modal space, in: Proceedings of the International Conference on Structural System Identification, Kassel, 2001, pp. 49-68 (Sections 3.4, 7.3).

[256] R. Williams, J. Crowley, H. Vold, The multivariate mode indicator function in modal analysis, in: Proceedings of the Fourth International Modal Analysis Conference, Los Angeles, 1986 (Sections 3.4, 7.3).

[257] J.R. Wright, J.E. Cooper, M. Desforges, Normal mode force appropriation - theory and application, Mechanical Systems and Signal Processing 13 (1999) 217-240 (Sections 3.4, 7.3).

[258] P. Atkins, J.R. Wright, K. Worden, An extension of force appropriation to the identification of non-linear multi-degree-of-freedom systems, Journal of Sound and Vibration 237 (2000) 23-43 (Sections 3.4, 7.3).

[259] M.F. Platten, J.R. Wright, J.E. Cooper, M. Sarmast, Identification of multi-degree-of-freedom non-linear simulated and experimental systems, in: Proceedings of the International Seminar on Modal Analysis (ISMA), Leuven, 2002, pp. 1195-1202 (Section 3.4).

[260] M.F. Platten, J.R. Wright, J.E. Cooper, Identification of a continuous structure with discrete non-linear components using an extended modal model, in: Proceedings of the International Seminar on Modal Analysis (ISMA), Leuven, 2004, pp. 2155-2168 (Section 3.4).

[261] S. Bellizzi, P. Gullemain, R. Kronland-Martinet, Identification of coupled non-linear modes from free vibration using time-frequency representation, Journal of Sound and Vibration 243 (2001) 191-213 (Sections 3.4, 3.5).

[262] T.K. Hasselman, M.C. Anderson, W.G. Gan, Principal component analysis for nonlinear model correlation, in: Proceedings of the 16th International Modal Analysis Conference, Santa Barbara, 1998, pp. 644-651 (Sections 3.4, 3.7, 7.4).

[263] V. Lenaerts, G. Kerschen, J.C. Golinval, Proper orthogonal decomposition for model updating of non-linear mechanical systems, Mechanical Systems and Signal Processing 15 (2001) 31-43 (Sections 3.4, 3.7).

[264] V. Lenaerts, G. Kerschen, J.C. Golinval, Identification of a continuous structure with a geometrical non-linearity, part II: proper orthogonal decomposition, Journal of Sound and Vibration 262 (2003) 907-919 (Sections 3.4, 3.7, 5.1, 7.4).

[265] P. Argoul, T.P. Le, T.M. Nguyen, Continuous wavelet transform for parameter identification from free decay responses of nonlinear structures, in: Proceedings of the EUROMECH Colloquium 457 on Nonlinear Modes of Vibrating Systems, Fréjus, 2004, pp. 73-78 (Section 3.4).

[266] M.B. Priestley, Power spectral analysis of nonstationary processes, Journal of Sound and Vibration 6 (1967) 86-97 (Section 3.5).

[267] J.K. Hammond, On the response of single and multidegree of freedom systems to nonstationary excitations, Journal of Sound and Vibration 7 (1968) 393-419 (Section 3.5).

[268] J.K. Hammond, P.R. White, The analysis of non-stationary signals using time-frequency methods, Journal of Sound and Vibration 190 (1996) 419-447 (Section 3.5). 
[269] K.C. Park, A. Robertson, K.F. Alvin, Identification of structural dynamic models using wavelet-generated impulse response data, Report CU-CAS-95-02, University of Colorado at Boulder, 1995 (Section 3.5).

[270] M. Ruzzene, A. Fasana, L. Garibaldi, B. Piombo, Natural frequencies and dampings identification using wavelet transform: application to real data, Mechanical Systems and Signal Processing 11 (1997) 207-218 (Section 3.5).

[271] W.J. Staszewski, Identification of damping in mdof systems using time-scale decomposition, Journal of Sound and Vibration 203 (1997) 283-305 (Section 3.5).

[272] P. Argoul, T.P. Le, Continuous wavelet transform for modal identification using free decay response, Journal of Sound and Vibration 277 (2004) 73-100 (Section 3.5).

[273] M. Boltezar, J. Slavic, Enhancements to the continuous wavelet transform for damping identifications on short signals, Mechanical Systems and Signal Processing 18 (2004) 1065-1076 (Section 3.5).

[274] D. Spina, C. Valente, G.R. Tomlinson, A new procedure for detecting nonlinearity from transient data using Gabor transform, Nonlinear Dynamics 11 (1996) 235-254 (Sections 3.5, 4.1).

[275] H. Franco, R.M.O. Pauletti, Analysis of nonlinear oscillations by gabor spectrograms, Nonlinear Dynamics 12 (1997) $215-236$ (Sections 3.5, 5.1).

[276] W.J. Staszewski, Analysis of non-linear systems using wavelets, Proceedings of the Institution of Mechanical Engineers Part CJournal of Mechanical Engineering Science 214 (2000) 1339-1353 (Sections 3.5, 4.1, 5.1, 6.4).

[277] M. Feldman, S. Braun, Identification of non-linear system parameters via the instantaneous frequency: application of the Hilbert transform and Wigner-Ville technique, in: Proceedings of the 13th International Modal Analysis Conference, Nashville, 1995, pp. 637-642 (Sections 3.5, 6.4).

[278] L. Wang, J. Zhang, C. Wang, S. Hu, Time-frequency analysis of nonlinear systems: the skeleton linear model and the skeleton curves, Journal of Vibration and Acoustics 125 (2003) 170-177 (Section 3.5).

[279] W.J. Staszewski, Identification of non-linear systems using multi-scale ridges and skeletons of the wavelet transform, Journal of Sound and Vibration 214 (1998) 639-658 (Section 3.5).

[280] L. Garibaldi, M. Ruzzene, A. Fasana, B. Piombo, Identification of non-linear damping mechanisms using the wavelet transform, Mecanique Industrielle et Materiaux 51 (1998) 92-94 (Section 3.5).

[281] P. Argoul, T.P. Le, Instantaneous indicators of structural behaviour based on the continuous cauchy wavelet analysis, Mechanical Systems and Signal Processing 17 (2003) 243-250 (Sections 3.5, 5.1).

[282] V. Lenaerts, G. Kerschen, J.C. Golinval, M. Ruzzene, E. Giorcelli, Validation of two nonlinear system identification techniques using an experimental testbed, Shock and Vibration 11 (2004) 365-375 (Section 3.5).

[283] Y. Kitada, Identification of nonlinear structural dynamic systems using wavelets, Journal of Engineering Mechanics 124 (1998) 1059-1066 (Section 3.5).

[284] R. Ghanem, F. Romeo, A wavelet-based approach for model and parameter identification of non-linear systems, International Journal of Non-Linear Mechanics 36 (2001) 835-859 (Section 3.5).

[285] S.L. Chen, K.C. Ho, Identification of nonlinear systems by Haar Wavelet, in: ASME International Mechanical Engineering Congress, Anaheim, 2004 (Section 3.5).

[286] L. Wang, J. Zhang, C. Wang, S. Hu, Identification of nonlinear systems through time-frequency filtering technique, Journal of Vibration and Acoustics 125 (2003) 199-204 (Section 3.5).

[287] G. Cybenko, Approximation by superpositions of a sigmoidal function, Mathematics of Control, Signals, and Systems 2 (1989) 303-314 (Section 3.6).

[288] J. Sjöberg, Q. Zhang, L. Ljung, A. Beneviste, B. Delyon, P.Y. Glorennec, H. Hjalmarsson, A. Juditsky, Nonlinear black-box modelling in system identification: a unified overview, Automatica 31 (1995) 1691-1724 (Section 3.6).

[289] A. Juditsky, H. Hjalmarsson, A. Beneviste, B. Delyon, L. Ljung, J. Sjöberg, Q. Zhang, Nonlinear black-box models in system identification: mathematical foundations, Automatica 31 (1995) 1725-1750 (Section 3.6).

[290] S.R. Chu, R. Shoureshi, M. Tenorio, Neural networks for system identification, IEEE Control Systems Magazine 10 (1990) 36-43 (Section 3.6).

[291] K.S. Narendra, K. Parthasarathy, Identification and control of dynamical systems using neural networks, IEEE Transactions on Neural Networks 1 (1990) 4-27 (Section 3.6).

[292] S. Chen, S.A. Billings, C.F.N. Cowan, P.M. Grant, Nonlinear-systems identification using radial basis functions, International Journal of Systems Science 21 (1990) 2513-2539 (Section 3.6).

[293] S.F. Masri, A.G. Chassiakos, T.K. Caughey, Structure-unknown non-linear dynamic systems: identification through neural networks, Smart Materials and Structures 1 (1992) 45-56 (Section 3.6).

[294] S.F. Masri, A.G. Chassiakos, T.K. Caughey, Identification of nonlinear dynamic systems using neural networks, Journal of Applied Mechanics 60 (1993) 123-133 (Section 3.6).

[295] K. Worden, G.R. Tomlinson, Modelling and classification of nonlinear systems using neural networks - I simulation, Mechanical Systems and Signal Processing 8 (1994) 319-356 (Section 3.6).

[296] K. Worden, G.R. Tomlinson, W. Lim, G. Sauer, Modelling and classification of non-linear systems using neural networks-II: a preliminary experiment, Mechanical Systems and Signal Processing 8 (1994) 395-419 (Sections 3.6, 5.1).

[297] A.G. Chassiakos, S.F. Masri, Modelling unknown structural systems through the use of neural networks, Earthquake Engineering and Structural Dynamics 25 (1996) 117-128 (Section 3.6).

[298] E.B. Kosmatopoulos, A.W. Smyth, S.F. Masri, A.G. Chassiakos, Robust adaptive neural estimation of restoring forces in nonlinear structures, Journal of Applied Mechanics 68 (2001) 880-893 (Sections 3.6, 5.1, 8). 
[299] J.S. Pei, A.W. Smyth, E.B. Kosmatopoulos, Analysis and modification of Volterra/Wiener neural networks for the adaptive identification of non-linear hysteretic dynamic systems, Journal of Sound and Vibration 275 (2004) 693-718 (Section 3.6).

[300] R. Le Riche, D. Gualandris, J.J. Thomas, F.M. Hemez, Neural identification of non-linear dynamic structures, Journal of Sound and Vibration 248 (2001) 247-265 (Section 3.6).

[301] Y. Song, C.J. Hartwigsen, D.M. McFarland, A.F. Vakakis, L.A. Bergman, Simulation of dynamics of beam structures with bolted joints using adjusted Iwan beam elements, Journal of Sound and Vibration 273 (2004) 249-276 (Sections 3.6, 3.7, 5.1,8).

[302] Y.C. Liang, D.P. Feng, J.E. Cooper, Identification of restoring forces in non-linear vibration systems using fuzzy adaptive neural networks, Journal of Sound and Vibration 242 (2001) 47-58 (Section 3.6).

[303] Y. Fan, C.J. Li, Non-linear system identification using lumped parameter models with embedded feedforward neural networks, Mechanical Systems and Signal Processing 16 (2002) 357-372 (Sections 3.6, 5.1, 8).

[304] S. Saadat, G.D. Buckner, T. Furukawa, M.N. Noori, An intelligent parameter varying approach for non-linear system identification of base excited structures, International Journal of Non-Linear Mechanics 39 (2004) 993-1004 (Section 3.6).

[305] M. Peifer, J. Timmer, H.U. Voss, Nonparametric identification of nonlinear oscillating systems, Journal of Sound and Vibration 267 (2003) 1157-1167 (Sections 3.6, 5.1, 8).

[306] G.P. Liu, S.A. Billings, V. Kadirkamanathan, Nonlinear system identification using wavelet networks, International Journal of Systems Science 31 (2000) 1531-1541 (Section 3.6).

[307] R. Babuska, H. Verbruggen, Neuro-fuzzy methods for nonlinear system identification, Annual Reviews in Control 27 (2003) $73-85$ (Section 3.6).

[308] O. Zienkiewicz, The Finite Element Method, McGraw-Hill, London, 1977 (Section 3.7).

[309] A. Berman, E.J. Nagy, Theory of incomplete models of dynamic structures, AIAA Journal 9 (1971) 1481-1487 (Section 3.7).

[310] M. Baruch, Optimization procedure to correct stiffness and flexibility matrices using vibration test, AIAA Journal 16 (1978) 1208-1210 (Section 3.7).

[311] H.G. Natke, Einfuhrung in Theorie und Praxis der Zeitreihen und Modalanalyse, Vieweg Verlag, Braunschweig/Wiesbaden, 1992 (Sections 3.7).

[312] M.I. Friswell, J.E. Mottershead, Finite Element Model Updating in Structural Dynamics, Kluwer Academic Publishers, London, 1995 (Sections 3.7, 7.4).

[313] M. Link, Updating of analytical models - basic procedures and extensions, in: J.M.M. Silva, N.M.M. Maia (Eds.), Modal Analysis \& Testing, Kluwer Academic Publishers, Dordrecht, 1999 (Sections 3.7, 7.4).

[314] R. Schmidt, Updating non-linear components, Mechanical Systems and Signal Processing 8 (1994) 679-690 (Section 3.7).

[315] R.K. Kapania, S. Park, Parametric identification of nonlinear structural dynamic systems using time finite element method, AIAA Journal 35 (1997) 719-726 (Section 3.7).

[316] K.D. Dippery, S.W. Smith, An optimal control approach to nonlinear system identification, in: Proceedings of the 16th International Modal Analysis Conference, Santa Barbara, 1998, pp. 637-643 (Section 3.7).

[317] A. Kyprianou, Non-linear parameter estimation of dynamic models using differential evolution: application to hysteretic systems and hydraulic engine mounts, Ph.D. Thesis, University of Sheffield, 1999 (Section 3.7).

[318] A. Kyprianou, K. Worden, M. Panet, Identification of hysteretic systems using the differential evolution algorithm, Journal of Sound and Vibration 248 (2001) 289-314 (Section 3.7).

[319] S. Meyer, M. Link, Modelling local non-linear behaviour-simultaneous updating of linear and non-linear parameters using frequency response residuals, in: Proceedings of International Conference on Structural Dynamics Modelling, Funchal, 2002 (Sections 3.7, 7.4).

[320] K.V. Yuen, J.L. Beck, Updating properties of nonlinear dynamical systems with uncertain input, Journal of Engineering Mechanics 129 (2003) 9-20 (Sections 3.7, 8).

[321] D.R. Mulville, Pyroshock Test Criteria, NASA Technical Standard, Report NASA-STD-7003, 1999 (Section 3.7).

[322] S. Doebling, F.M. Hemez, W. Rhee, Statistical model updating and validation applied to nonlinear transient structural dynamics, in: Proceedings of the European COST F3 Conference on System Identification \& Structural Health Monitoring, Madrid, 2000, pp. 409-418 (Section 3.7).

[323] X. Ma, A.F. Vakakis, Karhunen-Loève decomposition of the transient dynamics of a multibay truss, AIAA Journal 37 (1999) 939-946 (Section 3.7).

[324] G. Kerschen, On the model validation in non-linear structural dynamics, Ph.D. Thesis, University of Liège, 2003 (Sections 3.7, 7.4)

[325] G. Kerschen, J.C. Golinval, A model updating strategy of non-linear vibrating structures, International Journal for Numerical Methods in Engineering 60 (2004) 2147-2164 (Sections 3.7, 7.4).

[326] M.A. Kramer, Nonlinear principal component analysis using autoassociative neural networks, AIChE Journal 37 (1991) 233-243 (Section 3.7).

[327] D.C. Zimmerman, T. Hasselman, M. Anderson, Approximation and calibration of nonlinear structural dynamics, Nonlinear Dynamics 39 (2005) (Section 3.7).

[328] K. Wyckaert, Development and evaluation of detection and identification schemes for the nonlinear dynamical behaviour of mechanical structures, Ph.D. Thesis, Katholieke Universiteit Leuven, 1992 (Section 4.1).

[329] K. Vanhoenacker, J. Schoukens, J. Swevers, D. Vaes, Summary and comparing overview of techniques for the detection of nonlinear distortions, in: Proceedings of the International Seminar on Modal Analysis (ISMA), Leuven, 2002 (Section 4.1).

[330] G. Gloth, D. Göge, Handling of non-linear structural characteristics in ground vibration testing, in: Proceedings of the International Seminar on Modal Analysis (ISMA), Leuven, 2004 (Section 4.1). 
[331] J. Wong, J.E. Cooper, J.R. Wright, Detection and quantification of structural non-linearities, in: Proceedings of the International Seminar on Modal Analysis (ISMA), Leuven, 2002 (Section 4.1).

[332] D.E. Newland, An Introduction to Random Vibrations, Spectral and Wavelet Analysis, Prentice-Hall, Harlow, 1993 (Section 4.1 ).

[333] R.J. Allemang, D.L. Brown, Experimental modal analysis and dynamic component synthesis - vol. 2: measurement techniques for experimental modal analysis, AFWAL Technical Report TR 87-3069, 1987 (Section 4.1).

[334] M. Simon, G.R. Tomlinson, Use of the Hilbert transform in modal analysis of linear and non-linear structures, Journal of Sound and Vibration 96 (1984) 421-436 (Sections 4.1, 5.1).

[335] A. Rauch, Corehence: a powerful estimator of nonlinearity, theory and application, in: Proceedings of 10th International Modal Analysis Conference, San Diego, 1992, pp. 784-795 (Section 4.1).

[336] S.A. Billings, K.M. Tsang, Spectral analysis of block structured non-linear systems, Mechanical Systems and Signal Processing 4 (1990) 117-130 (Section 4.1).

[337] S.A. Billings, M.B. Fadzil, The practical identification of systems with nonlinearities, in: Proceedings of IFAC System Identification and Parameter Estimation, York, 1985 (Section 4.1).

[338] D. Choi, J. Chang, R.O. Stearman, E.J. Powers, Bispectral interaction of nonlinear mode interactions, in: Proceedings of the Second International Modal Analysis Conference, Orlando, 1984, pp. $602-609$ (Section 4.1).

[339] W.B. Collis, P.R. White, J.K. Hammond, Higher-order spectra: the bispectrum and trispectrum, Mechanical Systems and Signal Processing 12 (1998) 375-394 (Sections 4.1, 5.1).

[340] H. Van Der Auweraer, M. Mertens, W. Vancuyck, R. Snoeys, Recent trends in the analysis of nonlinear structures, in: Proceedings of the International Seminar on Modal Analysis (ISMA), Leuven, 1984 (Section 4.1).

[341] M. Mertens, H. Van Der Auweraer, P. Vanherck, R. Snoeys, Detection of nonlinear dynamic behaviour of mechanical structures, in: Proceedings of the Fourth International Modal Analysis Conference, Los Angeles, 1986 (Section 4.1).

[342] J. He, D.J. Ewins, A simple method of interpretation for the modal analysis of nonlinear systems, in: Proceedings of the 16th International Modal Analysis Conference, London, 1987, pp. 626-634 (Section 4.1).

[343] M. Mertens, H. Van Der Auweraer, P. Vanherck, R. Snoeys, The complex stiffness method to detect and identify non-linear dynamic behavior of SDOF systems, Mechanical Systems and Signal Processing 3 (1989) 37-54 (Section 4.1).

[344] W.J. Kim, S.J. Park, Non-linearity identification and quantification using an inverse Fourier transform, Mechanical System and Signal Processing 7 (1993) 239-255 (Section 4.1).

[345] D.E. Adams, R.J. Allemang, Residual frequency autocorrelation as an indicator of nonlinearity, International Journal of NonLinear Mechanics 36 (2000) 1197-1211 (Section 4.1).

[346] K. Vanhoenacker, T. Dobrowiecki, J. Schoukens, Design of multisine excitations to characterize the nonlinear distortions during FRF-measurements, IEEE Transactions on Instrumentation and Measurement 50 (2001) 1097-1102 (Section 4.1).

[347] P. Verboven, P. Guillaume, S. Vanlanduit, B. Cauberghe, Assessment of non-linear distortions in modal testing and analysis of vibrating automotive structures, Journal of Sound and Vibration (2005), in press (Section 4.1).

[348] I. Trendafilova, V. Lenaerts, G. Kerschen, J.C. Golinval, H. Van Brussel, Detection, localization and identification of nonlinearities in structural dynamics, in: Proceedings of the International Seminar on Modal Analysis (ISMA), Leuven, 2000 (Sections 4.1, 5.1).

[349] I. Trendafilova, H. Van Brussel, Non-linear dynamics tools for the motion analysis and condition monitoring of robot joints, Mechanical Systems and Signal Processing 15 (2001) 1141-1164 (Section 4.1).

[350] C.R. Farrar, H. Sohn, A.N. Robertson, Applications of nonlinear system identification to structural health monitoring, in: Proceedings of the Second European Workshop on Structural Health Monitoring, Munich, 2004 (Section 4.1).

[351] M. Simon, Developments in the modal analysis of linear and non-linear structures, Ph.D. Thesis, Victoria University of Manchester, 1983 (Section 4.2).

[352] I. Ahmed, Developments in Hilbert transform procedures with applications to linear and non-linear structures, Ph.D. Thesis, Victoria University of Manchester, 1987 (Section 4.2).

[353] N.E. King, K. Worden, An expansion technique for calculating Hilbert transforms, in: Proceedings of the Fifth International Conference on Recent Advances in Structural Dynamics, Southampton, 1994, pp. 1056-1065 (Section 4.2).

[354] P. Malatkar, A.H. Nayfeh, A plethora of nonlinear dynamics phenomena observed in a simple cantilever plate, in: Proceedings of the 2003 ASME Design Engineering Technical Conferences, Chicago, 2003, DETC2003/VIB-48619 (Sections 5.1, 8).

[355] R. Lin, D.J. Ewins, Location of localised stiffness non-linearity using measured modal data, Mechanical Systems and Signal Processing 9 (1995) 329-339 (Section 5.1).

[356] C.P. Fritzen, Damage detection based on model updating methods, Mechanical Systems and Signal Processing 12 (1998) 163-186 (Section 5.1).

[357] R. Pascual, I. Trendafilova, J.C. Golinval, W. Heylen, Damage detection using model updating and identification techniques, in: Proceedings of the Second International Conference on Identification in Engineering Systems, Swansea, 1999 (Section 5.1).

[358] S. Vanlanduit, P. Guillaume, J. Schoukens, K. Vanhoenacker, Detection and localization of nonlinearities using a scanner laser vibrometer, in: Proceedings of the International Seminar on Modal Analysis (ISMA), Leuven, 2000 (Section 5.1).

[359] A.F. Vakakis, D.J. Ewins, Effects of weak non-linearities on modal analysis, Mechanical Systems and Signal Processing 8 (1994) 175-198 (Section 5.1).

[360] J. Schoukens, Y. Rolain, J. Swevers, J. De Cuyper, Simple methods and insights to deal with non-linear distortions in FRFmeasurements, Mechanical Systems and Signal Processing 14 (2000) 657-666 (Section 5.1).

[361] A. Chatterjee, N.S. Vyas, Stiffness non-linearity classification through structured response component analysis using Volterra series, Mechanical Systems and Signal Processing 15 (2001) 323-336 (Section 5.1). 
[362] D.E. Adams, Frequency domain ARX models and multi-harmonic FRFs for nonlinear dynamic systems, Journal of Sound and Vibration 250 (2002) 935-950 (Section 5.1).

[363] R. Lind, K. Snyder, M. Brenner, Wavelet analysis to characterise non-linearities and predict limit cycles of an aeroelastic system, Mechanical Systems and Signal Processing 15 (2001) 337-356 (Section 5.1).

[364] P. Atkins, K. Worden, Identification of a multi-degree-of-freedom nonlinear system, in: Proceedings of the 15 th International Modal Analysis Conference, Orlando, 1997, pp. 1023-1028 (Section 5.1).

[365] D.E. Adams, R.J. Allemang, Polynomia,l non-polynomial, and orthogonal polynomial generating functions for nonlinear system identification, in: Proceedings of the International Seminar on Modal Analysis (ISMA), Leuven, 2000 (Section 5.1).

[366] A. Schmidt, L. Gaul, Implementation of constitutive laws with fractional derivatives in the finite elements method, Zeitschrift fur Angewandte Mathematik und Mechanik 83 (2003) 26-37 (Section 5.1).

[367] G. Kerschen, J.C. Golinval, F.M. Hemez, Bayesian model screening for the identification of non-linear mechanical structures, Journal of Vibration and Acoustics 125 (2003) 389-397 (Section 5.1).

[368] W.D. Iwan, A distributed element for hysteresis and its steady state dynamic response, Journal of Applied Mechanics 33 (1966) 893-900 (Section 5.1).

[369] W.D. Iwan, On a class of models for the yielding behavior of continuous and composite systems, Journal of Applied Mechanics 34 (1967) 612-617 (Section 5.1).

[370] R. Bouc, Forced vibrations of a mechanical system with hysteresis, in: Proceedings of the Fourth Conference on Non-linear Oscillations, Prague, 1967 (Sections 5.1, 6.1).

[371] Y.K. Wen, Method for random vibration of hysteretic systems, Journal of the Engineering Mechanics Division, Proceedings of the American Society of Civil Engineers 102 (1976) (Sections 5.1, 6.1).

[372] T.T. Baber, M. Noori, Random vibration of degrading pinching systems, Journal of Engineering Mechanics 111 (1985) 1010-1026 (Section 5.1)

[373] F. Vestroni, M. Noori, Hysteresis in mechanical systems - modeling and dynamic response, International Journal of Non-Linear Mechanics 37 (2002) 1261-1262 (Section 5.1).

[374] L.E. Goodman, A review of progress in analysis of interfacial slip damping, in: Proceedings of the ASME Annual Meeting, Atlantic City, 1959, pp. 35-48 (Section 5.1).

[375] M. Groper, Microslip and macroslip in bolted joint, Experimental Mechanics (1985) 172-174 (Section 5.1).

[376] L. Gaul, J. Lenz, Nonlinear dynamics of structures assembled by bolted joints, Acta Mechanica 125 (1997) 169-181 (Sections 5.1, 8).

[377] C.J. Hartwigsen, Y. Song, D.M. McFarland, L.A. Bergman, A.F. Vakakis, Experimental study of non-linear effects in a typical shear lap joint configuration, Journal of Sound and Vibration 277 (2004) 327-351 (Sections 5.1, 8).

[378] A. Ibrahim, F. Ismail, H.R. Martin, Modelling of the dynamics of continuous beam including nonlinear fatigue crack, International Journal of Analytical and Experimental Modal Analysis 2 (1987) 76-82 (Section 5.1).

[379] J.N. Sundermeyer, R.L. Weaver, On crack identification and characterization in a beam by non-linear vibration analysis, Journal of Sound and Vibration 183 (1995) 857-871 (Section 5.1).

[380] M.I. Friswell, J.E.T. Penny, Crack modeling for structural health monitoring, Structural Health Monitoring 1 (2002) 139-148 (Section 5.1).

[381] E. Luzzato, Approximate computation of non-linear effects in a vibrating cracked beam, Journal of Sound and Vibration 265 (2003) 745-763 (Section 5.1).

[382] W. Liu, G. Tomlinson, K. Worden, Nonlinear study of particle dampers, in: Proceedings of the International Seminar on Modal Analysis (ISMA), Leuven, 2002 (Section 5.1).

[383] C.X. Wong, N. Tassini, S. Patsias, K. Worden, Identification of a beam with a distributed friction nonlinearity, in: Proceedings of the International Seminar on Modal Analysis (ISMA), Leuven, 2004 (Section 5.1).

[384] K. Worden, G.R. Tomlinson, Parametric and nonparametric identification of automotive shock absorbers, in: Proceedings of 10th International Modal Analysis Conference, San Diego, 1992, pp. $764-765$ (Sections 5.2, 6.1).

[385] R. Sibson, Manual for the TILE4 Interpolation Package, Department of Mathematics and Statistics, University of Bath, 1985 (Section 6.1).

[386] K. Worden, G.R. Tomlinson, Application of the restoring force method to nonlinear elements, in: Proceedings of Seventh International Modal Analysis Conference, Las Vegas, 1989 (Section 6.1).

[387] M. Yar, J.K. Hammond, Parameter estimation for hysteretic systems, Journal of Sound and Vibration 117 (1987) 161-172 (Section 6.1).

[388] K. Worden, G.R. Tomlinson, An experimental study of a number of nonlinear SDOF systems using the restoring force surface method, in: Proceedings of the Ninth International Modal Analysis Conference, Las Vegas, 1991, pp. $757-764$ (Section 6.1).

[389] N. Hunter, T. Paez, D.L. Gregory, Force-state mapping using experimental data, in: Proceedings of the Seventh International Modal Analysis Conference, Los Angeles, 1989, pp. 843-869 (Section 6.1).

[390] M.D. Allen, Mean-square error of prediction as a criterion for selecting variables, Technometrics 13 (1971) $469-475$ (Section 6.1).

[391] K. Worden, G.R. Tomlinson, Identification of linear/nonlinear restoring force surfaces in single- and multi-mode systems, in: Proceedings of the Third International Conference on Recent Advances in Structural Dynamics, Southampton, 1988, pp. 299-308 (Section 6.2).

[392] M.A Al-Hadid, Identification of nonlinear dynamic systems using the force-state mapping technique, Ph.D. Thesis, University of London, 1989 (Section 6.1, 6.2).

[393] K. Worden, Parametric and nonparametric identification of nonlinearity in structural dynamics, Ph.D. Thesis, Department of Mechanical Engineering, Heriot-Watt University, 1989 (Section 6.2).

[394] J.R. Wright, M.A. Al-Hadid, Sensitivity of the force-state mapping approach to measurement errors, International Journal of Analytical and Experimental Modal Analysis 6 (1991) 89-103 (Section 6.2). 
[395] K. Worden, J.R. Wright, M.A. Al-Hadid, Experimental identification of multi-degree-of-freedom nonlinear-systems using restoring force methods, International Journal of Analytical and Experimental Modal Analysis 9 (1994) 35-55 (Section 6.2).

[396] Y.C. Liang, J. Cooper, Physical parameter identification of distributed systems, in: Proceedings of the 10th International Modal Analysis Conference, San Diego, 1992, pp. 1334-1340 (Section 6.2).

[397] G.F. Simmons, Introduction to Topology and Modern Analysis, McGraw-Hill, New York, 1963 (Section 6.3).

[398] S.A. Billings, S. Chen, Extended model set, global data and threshold model identification of severely non-linear systems, International Journal of Control 50 (1989) 1897-1923 (Section 6.3).

[399] S.A. Billings, H.B. Jamaluddin, S. Chen, A comparison of the backpropagation and recursive prediction error algorithms for training neural networks, Mechanical Systems and Signal Processing 5 (1991) 233-255 (Section 6.3).

[400] S. Chen, S.A. Billings, W. Luo, Orthogonal least-squares methods and their application to nonlinear system identification, International Journal of Control 50 (1989) 1873-1896 (Section 6.3).

[401] M. Feldman, Investigation of the natural vibrations of machine elements using the Hilbert transform, Soviet Machine Science 2 (1985) 44-47 (Section 6.4).

[402] F. Brancaleoni, D. Spina, C. Valente, A free oscillation based technique for the identification of nonlinear dynamic systems, in: Proceedings of 13th IMACS, Dublin, 1992 (Section 6.4).

[403] M.G. Sainsbury, Y.K. Ho, Application of the time domain Fourier filter output method to the identification of a lightly damped non-linear system with an odd-spring characteristic, Mechanical Systems and Signal Processing 15 (2001) $357-366$ (Section 6.4).

[404] V. Volterra, Theory of Functionals and Integral Equations, Dover Publications, New York, 1959 (Section 6.5).

[405] J.F. Barrett, The use of functionals in the analysis of nonlinear systems, Journal of Electronics and Control 15 (1963) 567-615 (Section 6.5).

[406] K. Worden, G. Manson, Random vibrations of a duffing oscillator using the Volterra series, Journal of Sound and Vibration 217 (1998) 781-789 (Section 6.5).

[407] K. Worden, G. Manson, Random vibrations of a multi-degree-of-freedom non-linear system using the Volterra series, Journal of Sound and Vibration 226 (1999) 397-405 (Section 6.5).

[408] K. Worden, G. Manson, A Volterra series approximation to the coherence of the Duffing oscillator, Journal of Sound and Vibration 286 (2005) 529-547 (Section 6.5).

[409] S.J. Gifford, G.R. Tomlinson, Recent advances in the application of functional series to non-linear structures, Journal of Sound and Vibration 135 (1989) 289-317 (Section 6.5).

[410] J. Wray, G.G.R. Green, Calculation of the Volterra kernels of nonlinear dynamic systems using an artificial neural network, Biological Cybernetics 71 (1994) 187-195 (Section 6.5).

[411] J.C. Peyton Jones, S.A. Billings, Recursive algorithm for computing the frequency response of a class of non-linear difference equation models, International Journal of Control 50 (1989) 1925-1940 (Section 6.5).

[412] J.E. Chance, K. Worden, G.R. Tomlinson, Frequency domain analysis of NARX neural networks, Journal of Sound and Vibration 213 (1998) 915-941 (Section 6.5).

[413] T.J. Dodd, C.J. Harris, Identification of nonlinear time series via kernels, International Journal of System Science 33 (2002) 737-750 (Section 6.5).

[414] T.J. Dodd, R.F. Harrison, Estimating Volterra filters in Hilbert spaces, in: Proceedings of the 2002 IFAC World Congress, Barcelona, 2002 (Section 6.5).

[415] T.J. Dodd, R.F. Harrison, A new solution to Volterra series estimation, in: Proceedings of the 2002 IFAC World Congress, Barcelona, 2002 (Section 6.5).

[416] Y. Wan, T.J. Dodd, R.F. Harrison, A kernel method for non-linear systems identification-infinite degree Volterra series estimation, Research Report 842, Department of Automatic Control and Systems Engineering, The University of Sheffield, 2003 (Section 6.5).

[417] W.H. Press, B.P. Flannery, S.A. Teukolsky, W.T. Vetterling, Numerical Recipes-The Art of Scientific Computing, Cambridge University Press, Cambridge, 1986 (Section 6.5).

[418] K.M. Tsang, S.A. Billings, Frequency Response Extraction and Plotting Package, Department of Automatic Control and Systems Engineering, University of Sheffield, 1988 (Section 6.5).

[419] D.M. Storer, G.R. Tomlinson, Higher order frequency response functions and their relation to practical structures, in: Proceedings of the Ninth International Modal Analysis Conference, Firenze, 1991 (Section 6.5).

[420] H. Liu, T. Vinh, T. Chouychai, M. Djouder, Second order transfer function: computation and physical interpretation, in: Proceedings of the Fifth International Modal Analysis Conference, London, 1987 (Section 6.5).

[421] K.M. Tsang, S.A. Billings, Reconstruction of linear and non-linear continuous time models from discrete time sampled-data systems, Mechanical Systems and Signal Processing 6 (1992) 69-84 (Section 6.5).

[422] J.R.F. Arruda, J.M.C. Santos, Mechanical joint parameter estimation using frequency response functions and component mode synthesis, Mechanical Systems and Signal Processing 7 (1993) 493-508 (Section 7.1).

[423] E. Balmès, A finite element updating procedure using frequency response functions; application to the MIT/SERC interferometer testbed, in: Proceedings of the 11th International Modal Analysis Conference, Kissimmee, 1993, pp. 176-182 (Section 7.1).

[424] R. Lin, D.J. Ewins, Analytical model improvement using frequency response functions, Mechanical Systems and Signal Processing 8 (1994) 437-458 (Section 7.1).

[425] G. Strang, Introduction to Applied Mathematics, Wellesley-Cambridge Press, Cambridge, 1986 (Section 7.2).

[426] G. Kerschen, J.C. Golinval, Frequency domain approaches for the identification of an experimental beam with a local non-linearity, in: Proceedings of the 20th International Modal Analysis Conference, Los Angeles, 2002, pp. 331-337 (Section 7.2). 
[427] S. Naylor, M.F. Platten, J.R. Wright, J.E. Cooper, Identification of multi-degree-of-freedom systems with nonproportional damping using the resonant decay method, Journal of Vibration and Acoustics 126 (2004) 298-306 (Section 7.3).

[428] Y. Ben-Haim, Identification of certain polynomial nonlinear structures by adaptive selectively sensitive excitation, Journal of Vibration and Acoustics 115 (1993) 246-255 (Section 7.3).

[429] C. Mares, J.E. Mottershead, M.I. Friswell, Results obtained by minimising natural frequency errors and using physical reasoning, Mechanical Systems and Signal Processing 17 (2003) 39-46 (Section 7.4).

[430] P. Holmes, J.L. Lumley, G. Berkooz, Turbulence, Coherent Structures, Dynamical Systems and Symmetry, Cambridge, New York, 1996 (Section 7.4).

[431] G. Kerschen, J.C. Golinval, A.F. Vakakis, L.A. Bergman, The method of proper orthogonal decomposition for dynamical characterization and order reduction of mechanical systems: an overview, Nonlinear Dynamics 41 (2005) 147-170 (Section 7.4).

[432] F.M. Hemez, Y.W. Ben-Haim, Info-gap robustness for the correlation of tests and simulations of a non-linear transient, Mechanical Systems and Signal Processing 18 (2004) 1443-1467 (Section 7.4).

[433] J.L. Quinby, B.F. Feeny, Low-frequency phenomena in a frictionally excited beam, ASME International Mechanical Engineering Congress, Anaheim, 2004 (Section 8).

[434] J.P. Caffrey, S.F. Masri, F. Tasbihgoo, A.W. Smyth, A.G. Chassiakos, A re-configurable test apparatus for complex nonlinear dynamic systems, Nonlinear Dynamics 36 (2004) 181-201 (Section 8).

[435] J.K. Hammond, Non-structural dynamics in structural dynamics @ 2000: current status and future directions, in: D.J. Ewins, D. Inman (Eds.), Research Studies Press, Baldock, 2001 (Section 8).

[436] D. Quinn, R. Rand, J. Bridge, The dynamics of resonance capture, Nonlinear Dynamics 8 (1995) 1-20 (Section 8).

[437] E. Pesheck, C. Pierre, S.W. Shaw, Accurate reduced-order models for a simple rotor blade model using nonlinear normal modes, Mathematical and Computer Modelling 33 (2001) 1085-1097 (Section 8).

[438] E. Pesheck, C. Pierre, S.W. Shaw, Modal reduction of a nonlinear rotating beam through nonlinear normal modes, Journal of Vibration and Acoustics 124 (2002) 229-236 (Section 8).

[439] E. Pesheck, C. Pierre, S.W. Shaw, A new Galerkin-based approach for accurate non-linear normal modes through invariant manifolds, Journal of Sound and Vibration 249 (2002) 971-993 (Section 8).

[440] P. Apiwattanalunggarn, S.W. Shaw, C. Pierre, Finite-element-based nonlinear modal reduction of a rotating beam with largeamplitude motion, Journal of Vibration and Control 9 (2003) 235-263 (Section 8).

[441] B.F. Feeny, R. Kappagantu, On the physical interpretation of proper orthogonal modes in vibrations, Journal of Sound and Vibration 211 (1998) 607-616 (Section 8).

[442] B.F. Feeny, On the proper orthogonal modes and normal modes of continuous vibration systems, Journal of Vibration and Acoustics 124 (2002) 157-160 (Section 8).

[443] G. Kerschen, J.C. Golinval, Physical interpretation of the proper orthogonal modes using the singular value decomposition, Journal of Sound and Vibration 249 (2002) 849-865 (Section 8).

[444] G. Kerschen, J.C. Golinval, Feature extraction using auto-associative neural networks, Smart Materials and Structures 13 (2004) 211-219 (Section 8).

[445] A.W. Smyth, S.F. Masri, E.B. Kosmatopoulos, A.G. Chassiakos, T.K. Caughey, Development of adaptive modeling techniques for non-linear hysteretic systems, International Journal of Non-Linear Mechanics 37 (2002) 1437-1451 (Section 8).

[446] J.N. Yang, S. Lin, On-line identification of non-linear hysteretic structures using an adaptive tracking technique, International Journal of Non-Linear Mechanics 39 (2004) 1481-1491 (Section 8). 